\title{
Untersuchung des elektrischen Widerstandsschaltens perowskitischer Manganatsysteme auf der Nanometerskala
}

\section{Dissertation}

zur Erlangung des mathematisch-naturwissenschaftlichen Doktorgrades „Doctor rerum naturalium“

der Georg-August-Universität Göttingen

vorgelegt von

Jon-Olaf Krisponeit

aus Bremen

Göttingen 2011

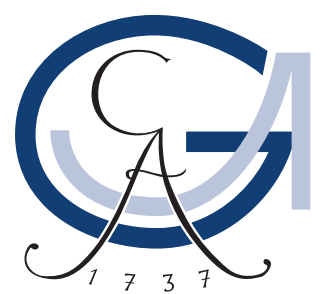


Referent: Prof. Konrad Samwer

Koreferent: Prof. Christian Jooss

Tag der mündlichen Prüfung: 13. Dezember 2011 
Phantasie haben heißt nicht, sich Dinge ausdenken.

Es heißt sich etwas aus den Dingen machen.

Meerfahrt mit Don Quijote ThOMAS MANN 

$\begin{array}{lr}\text { Einleitung } & 1\end{array}$

1. Manganate 3

1.1. Kristallstruktur . . . . . . . . . . . . . . . . . . . 5 5

1.2. Elektronische und magnetische Eigenschaften . . . . . . . . . . . 6

1.2.1. Kristallfeldaufspaltung und Jahn-Teller-Effekt . . . . . . . . . . . . . 6

1.2.2. Transportmechanismen und Magnetismus . . . . . . . . . . . . 8

1.3. Polaronen und Ladungsordnung . . . . . . . . . . . . . . . . . . . . 10

1.4. Phasendiagramme . . . . . . . . . . . . . . . . . . . . . 12

1.5. Phasenseparation und Perkolation . . . . . . . . . . . . 13

1.6. Manganatoberflächen . . . . . . . . . . . . . . . . . . . 17

2. Elektrisches Widerstandsschalten 19

2.1. Phänomenologische Einordnung . . . . . . . . . . . . . . . . . . . 20

2.2. Anwendungsmöglichkeiten . . . . . . . . . . . . . . . . . 21

2.3. Historischer Abriss . . . . . . . . . . . . . . . . . . . . . 21

2.4. Übergangsmetalloxide als memristive Systeme . . . . . . . . . . . . . . . . . 23

2.5. Erklärungsansätze . . . . . . . . . . . . . . . . . . . 23

2.5.1. Filamentäre Schalteffekte . . . . . . . . . . . . . . 23

2.5.2. Schalten an Grenz- oder Kontaktflächen . . . . . . . . . . . . . . . . 24

3. Experimentelle Methoden $\quad 27$

3.1. Metallorganische Aerosoldeposition (MAD) . . . . . . . . . . . 27

3.2. Charakterisierung . . . . . . . . . . . . . . . . . . . 28

3.2.1. Röntgenstrukturanalyse . . . . . . . . . . . . . . 28

3.2.2. Transportmessungen . . . . . . . . . . . . . . . 30

3.3. Atomare Kraftmikroskopie $(\mathrm{AFM}) \quad \ldots \ldots \ldots$

3.3.1. Grundlagen . . . . . . . . . . . . . . . . 30

3.3.2. Annäherung der AFM-Spitze zum Kontaktmodus . . . . . . . . . . . 31 
3.3.3. Die C-AFM-Methode . . . . . . . . . . . . . . . . . . . 33

3.4. Vorstellung der verwendeten UHV-Apparatur . . . . . . . . . . . . . 35

3.5. Abschätzung des minimalen Kontaktradius . . . . . . . . . . . . . . . . 39

4. Ergebnisse 41

4.1. Struktur . . . . . . . . . . . . . . . . . . . . 42

4.2. Makroskopische elektronische Eigenschaften . . . . . . . . . . . . . . . . 43

4.3. Oberflächenmorphologie . . . . . . . . . . . . . . . . . . 44

4.4. Resistives Schalten: Charakterisierung des Effektes . . . . . . . . . . . . 45

4.5. Ortsaufgelöste Messungen . . . . . . . . . . . . . . . . . . . . . 49

4.5.1. Stromkarten elektrisch induzierter Widerstandsänderungen . . . . . 49

4.5.2. Oberflächenmodifikationen . . . . . . . . . . . . . . . . 53

4.5.3. Domänenwachstum in Abhängigkeit von Spannung und Pulsdauer . 54

4.6. Zeitabhängige Messungen . . . . . . . . . . . . . . . . . . 57

4.6.1. Rückbildung einer metallischen Domäne . . . . . . . . . . . . . . 57

4.6.2. Leitfähigkeitsanstiegs- und -abklingverhalten in Pulsserienversuchen 59

4.7. Ergänzungen: Probenspezifische Untersuchungen . . . . . . . . . . . . . 62

4.7.1. Untersuchung einer LMO-terminierten Schicht . . . . . . . . . . . . 62

4.7.2. Diskrete Leitfähigkeitswerte einer LSMO-Oberfläche . . . . . . . . . 64

4.8. Zusammenfassung . . . . . . . . . . . . . . . 68

5. Diskussion 71

5.1. Modell: Elektrisch induzierter struktureller Übergang . . . . . . . . . . . . . 72

5.1.1. Motivation . . . . . . . . . . . . . . . 72

5.1.2. Vorüberlegungen . . . . . . . . . . . . . . . . 74

5.1.3. Modellbildung . . . . . . . . . . . . . . . . . . . . . 79

5.1.4. Offene Fragen . . . . . . . . . . . . . . . . . . . . . . 81

5.2. Vergleich mit anderen Modellen . . . . . . . . . . . . . . . . . . 83

5.2.1. Schottky-Barriere . . . . . . . . . . . . . . . 83

5.2.2. Sauerstoffleerstellenmigration . . . . . . . . . . . . . 84

5.3. Stromdichten und Heizeffekte . . . . . . . . . . . . . . . . . 86

5.3.1. Ausgangszustand vor dem erstmaligen Schalten . . . . . . . . . . . . 86

5.3.2. Metallischer Zustand (LRS) . . . . . . . . . . . . . . . . 87

5.3.3. Isolierender Zustand $(\mathrm{HRS}) \ldots \ldots \ldots$. . . . . . . . . . 88

5.3.4. Morphologische Änderungen . . . . . . . . . . . . . . . . . . . . . . . 88

5.4. Domänenkriechen . . . . . . . . . . . . . . . . . . . . . . . 90

5.4.1. Analyse des Domänenwachstums in Abhängigkeit von den Pulsparametern . . . . . . . . . . . . . . . . . 99 90

5.4.2. Anwendung auf die experimentellen Ergebnisse . . . . . . . . . . . 94

5.5. Einfluss von undotierten Decklagen . . . . . . . . . . . . . . . . . 97

5.6. Diskrete Leitfähigkeitsplateaus . . . . . . . . . . . . . . . . . . . . . . 99

6. Zusammenfassung und Ausblick 103

6.1. Zusammenfassung . . . . . . . . . . . . . . . . . 103

6.2. Anwendungsrelevanz . . . . . . . . . . . . . . . . . . . . 104 
6.3. Ausblick . . . . . . . . . . . . . . . . . . . . . . . 105

$\begin{array}{ll}\text { A. Widerstands-Kapazitäts-Netzwerke } & 107\end{array}$

A.1. Motivation . . . . . . . . . . . . . . . . . . . . . . . . . . . 107

A.2. Knotenpotentialanalyse . . . . . . . . . . . . . . . . . . 109

A.3. Zufallsnetzwerke . . . . . . . . . . . . . . . . . . . . . . . 109

A.4. Random-Field Ising Modell . . . . . . . . . . . . . . . . . . . . . . 113

A.5. Fazit . . . . . . . . . . . . . . . . . . . . 116

$\begin{array}{ll}\text { Abbildungsverzeichnis } & 117\end{array}$

$\begin{array}{ll}\text { Literatur } & 119\end{array}$

$\begin{array}{ll}\text { Veröffentlichungen } & 131\end{array}$

$\begin{array}{ll}\text { Danksagung } & 133\end{array}$

$\begin{array}{ll}\text { Lebenslauf } & 135\end{array}$ 



\section{Einleitung}

Unser Alltag ist heute wie nie zuvor von der schnellen Verfügbarkeit der verschiedensten Informationen geprägt. Die Bewahrung, Weitergabe und Verbreitung von Daten, welche unser Zivilisationsniveau in technischer wie auch intellektueller und musischer Hinsicht dokumentieren, ist ein Urbedürfnis des Menschen. Bei der Weiterentwicklung der verwendeten Speichermedien wird vor allem der stetig wachsenden Datenmenge mit fortschreitender Miniaturisierung begegnet und eine Reduktion der Zugriffszeiten sowie verbesserte Langzeitstabilität angestrebt - von prähistorischen Felszeichnungen über Papyrus, Pergament, Film- und Mikrofilm bis hin zu Magnetbändern und -platten.

Während die Notwendigkeit immer größerer Datendichten bald nicht nur an technologische, sondern sogar an physikalische Grenzen stößt, treten jedoch zunehmend auch ökologische Aspekte wie eine geringe Leistungsaufnahme in den Vordergrund. Die Suche nach neuen Funktionsmaterialien und die Entwicklung darauf basierender neuartiger Speicherkonzepte, welche unseren modernen Anforderungen gerecht werden können, ist daher ein reges und bedeutendes Betätigungsfeld.

Die hier untersuchte Familie der perowskitischen Manganoxide, gebräuchlicherweise als "Manganate" bezeichnet, ist für die Wissenschaft ebenso faszinierend wie herausfordernd. Ihre phänomenologische Vielfalt - insbesondere durch verschiedene Stimuli getriebene Widerstandseffekte - erwächst aus einem sehr komplex wechselwirkenden mikroskopischen Gefüge. Die üblichen physikalischen Abstraktionsansätze, wie vor allem eine getrennte Betrachtung des Elektronen-, Spin- und Gittersystems, werden diesen hochkorrelierten Materialien im Allgemeinen nicht gerecht. Obwohl anfangs inbesondere das kolossale Magnetowiderstandsverhalten ${ }^{[1]}$ die Hoffnung auf eine industrielle Verwendbarkeit in der Sensorik zur Auslesung magnetischer Speichermedien nährte, wurde ihnen bis heute nur eine Nischenbedeutung für bolometrische Anwendungen oder als Kathodenmaterial in Feststoffbrennstoffzellen zuteil.

Zukünftige Anwendungen für Manganate könnten sich aufgrund ihrer vollständigen Spinpolarisation ${ }^{[2]}$ in spinelektronischen Bauelementen ergeben. Darüberhinaus reihen sich 
Manganate jedoch auch in die Vielzahl jener oxidischen Systeme ein, welche durch elektrische Spannungen oder Ströme resistiv geschaltet werden können. ${ }^{[3]}$

Derartige Effekte sind nicht nur im Kontext der memristiven Systeme ${ }^{[4,5]}$ von fundamentaler konzeptioneller Bedeutung, sondern offenbaren auch ein großes Potential für Speicherelemente in Form des RRAM („,resistive random access memory"). ${ }^{[6-8]}$ Eine industrielle Umsetzung dieses vielversprechenden Konzeptes, welches einmal den gegenwärtig sehr verbreiteten Flash-Speicher ablösen könnte, wird jedoch durch das mangelnde physikalische Verständnis der zugrundeliegenden Schalteffekte noch sehr erschwert. Zur gezielten Verbesserung der Schalteigenschaften - also hin zu großen Datendichten, niedrigen Schaltspannungen oder -strömen, kurzen Schaltzeiten und langlebigen Widerstandszuständen ist die Entwicklung eines mikroskopischen Bildes der Schaltphänomene unabdingbar.

Der Hauptteil dieser Arbeit befasst sich mit der rastersondenmikroskopischen Untersuchung des Widerstandsschaltens unter elektrischer Spannung. Für Manganate wurden bisher nur sehr wenige Studien des Schalteffektes auf kleiner Längenskala veröffentlicht. ${ }^{[9]}$ Daher soll hier ein Beitrag geleistet werden, der die verbreiteten Messungen unter makroskopischer Kontaktierung ergänzt: Es werden nicht nur die gemittelten Schalteigenschaften einer großen Kontaktfläche, welche vielleicht nur von einem kleinen Bruchteil individuell schaltender Regionen dominiert sind, erfasst. Stattdessen wird die Ausbreitung der elektrisch induzierten Widerstandsänderung einzelner Domänen auf der Nanometerskala abgebildet.

Nach einem Überblick der wichtigsten experimentellen Charakteristika der untersuchten Manganatfilme und der Konzepte zu ihrer Beschreibung folgt eine Einführung in die resistiven Schalteffekte an Manganaten und anderen Oxiden. Die verwendeten experimentellen Methoden werden in Kapitel 3 vorgestellt. Der Ergebnisteil beginnt mit einer allgemeinen Charakterisierung der untersuchten Proben, es folgen Messungen der zentralen elektrischen Eigenschaften des Schalteffektes. Danach werden räumlich und zeitlich aufgelöste Messungen gezeigt und komplexere, probenspezifische Beobachtungen dargestellt. In Kapitel 5 wird nach einer Einordnung der experimentellen Resultate zunächst ein strukturelles Modell zu ihrer Beschreibung eingeführt und anderen Erklärungsansätzen gegenübergestellt. Den Abschluss des Hauptteils bildet eine Zusammenfassung der wesentlichen Erkenntnisse.

Im Anhang wird ein numerisches Simulationsverfahren erläutert, das ebenfalls im Rahmen dieses Dissertationsvorhabens - aber von den obigen Bemühungen unabhängig - entwickelt wurde. Wie in früheren Studien ${ }^{[10,11]}$ wird die Phasenseparation von Manganaten dabei durch Widerstandsnetzwerke dargestellt. Es findet jedoch eine Ergänzung durch kapazitive Komponenten statt, um auch Besonderheiten im frequenzabhängigen Transportverhalten zu beleuchten. Die im Anhang beschriebene Methode stellt zwar nur einen ersten Ansatz dar, vermag aber bereits einige Messergebnisse mit geringem Aufwand qualitativ zu reproduzieren. 


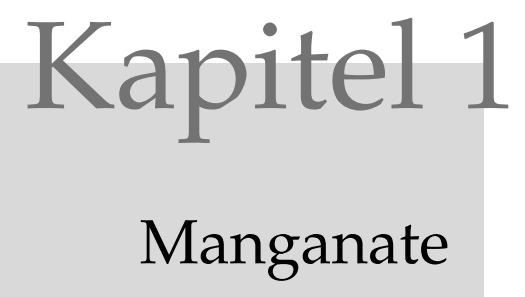

Manganate zeigen eine Vielzahl interessanter Eigenschaften und Effekte sowie sehr reichhaltige Phasendiagramme, die in der subtilen mikroskopischen Balance der elektronischen, magnetischen und elastischen Wechselwirkungen begründet sind. Sie verfügen dadurch nicht nur über technisches Anwendungspotential in der Sensorik und Spinelektronik, sondern sind als gemischtvalente, stark korrelierte Elektronensysteme mit kompositionskontrollierter Elektron-Phonon-Kopplung auch von hohem physikalischem Interesse.

Die frühen Untersuchungen polykristalliner Manganatproben durch Jonker \& van Santen lieferten neben der Kristallstruktur auch schon erste Erkenntnisse über die magnetischen ${ }^{[12]}$ und elektrischen ${ }^{[13]}$ Eigenschaften. Nahe der Curie-Temperatur $T_{\mathrm{C}}$ wurden dabei erste Indizien eines später als Metall-Isolator-Übergang identifizierten Widerstandsverlaufes festgestellt.

Umfangreiche Neutronen- und Röntgenbeugungsarbeiten von Wollan \& Koehler ergaben außerdem frühzeitig Einblicke in komplexe magnetische Phasendiagramme, die für tiefe Temperaturen Ferromagnetismus, verschiedene antiferromagnetische Ordnungen und sogar Bereiche einer Phasenkoexistenz von beidem enthalten. ${ }^{[14]}$

Volger wies 1954 auf negative Magnetowiderstandseffekte in der Nähe der Curie-Temperatur hin; ${ }^{[15]}$ spätere Studien an Manganateinkristallen, beispielsweise von Searle \& Wang zeigten ähnliche Resultate. ${ }^{[16,17]}$ Die Tragweite dieser Effekte, insbesondere auch im Hinblick auf mögliche Anwendungen, wurde dabei jedoch vorerst unterschätzt.

Die heutige, intensive Erforschung der Manganate wurde durch den Fund dramatischer Magnetowiderstandsphänomene in dünnen Manganatfilmen durch von Helmolt et al. ${ }^{[1]}$, Chahara et al. ${ }^{[18]}$ und Kusters et al. ${ }^{[19]}$ eingeleitet. Dieser sogenannte CMR-Effekt (Colossal Magnetoresistance) ist in Abb. 1.1 exemplarisch für einen $\mathrm{La}_{0,75} \mathrm{Ca}_{0,25} \mathrm{MnO}_{3}$-Film dargestellt: Ohne anliegendes Magnetfeld ist ein scharfer Metall-Isolator-Übergang im $R(T)$-Diagramm ersichtlich, welcher sich bei $T_{\mathrm{MI}}$ nahe der Curie-Temperatur $T_{\mathrm{C}}$ ereignet. Im Magnetfeld von $B=5 \mathrm{~T}$ findet - insbesondere in der Umgebung des Übergangs eine deutliche Verringerung des Widerstandes statt. Diese lässt sich durch den Ausdruck $C M R=\frac{R(0)-R(H)}{R(0)} \times 100 \%$ quantifizieren und beläuft sich im gezeigten Beispiel auf etwa

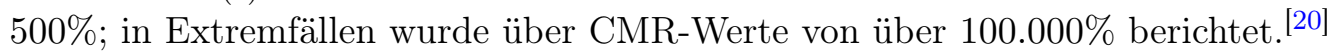

Das vorliegende einführende Kapitel gibt einen Überblick über die wichtigsten Eigenschaften der hier untersuchten perowskitischen Manganatfilme und erläutert die für ihre 
Beschreibung etablierten Konzepte. Aufgrund der faszinierenden phänomenologischen Vielfalt dieser Materialien einerseits und des ihr zugrundeliegenden komplexen Wechselspiels des elektronischen und strukturellen Systems andererseits muss hier eine enge Beschränkung auf die zentralen Aspekte vorgenommen werden.

Es wird dabei zunächst die Kristallstruktur dargestellt, es folgen die damit eng verknüpften elektrischen und magnetischen Eigenschaften. Dabei erfolgt eine qualitative Beschreibung der wichtigsten Transportmechanismen, um danach ein phänomenologisches Verständnis für den CMR-Effekt und die Phasendiagramme von Manganaten zu vermitteln. Anschließend werden Aspekte wie die polaronische Natur der Ladungsträger und Perkolationseffekte knapp dargestellt. Den Abschluss bildet eine Zusammenfassung von im Rahmen dieser Arbeit relevanten Erkenntnissen über die Ober- und Grenzflächen von Manganaten.

Für umfassendere Einführungen sei an dieser Stelle exemplarisch auf die Bücher von Dagotto $^{[21]}$ und Tokura ${ }^{[22]}$ sowie die Übersichtsartikel von Coey et al. ${ }^{[23]}$, Salamon \& Jaime $^{[24]}$, Dagotto et al. ${ }^{[25,26]}$ und Moshnyaga et al. ${ }^{[27,28]}$ verwiesen.

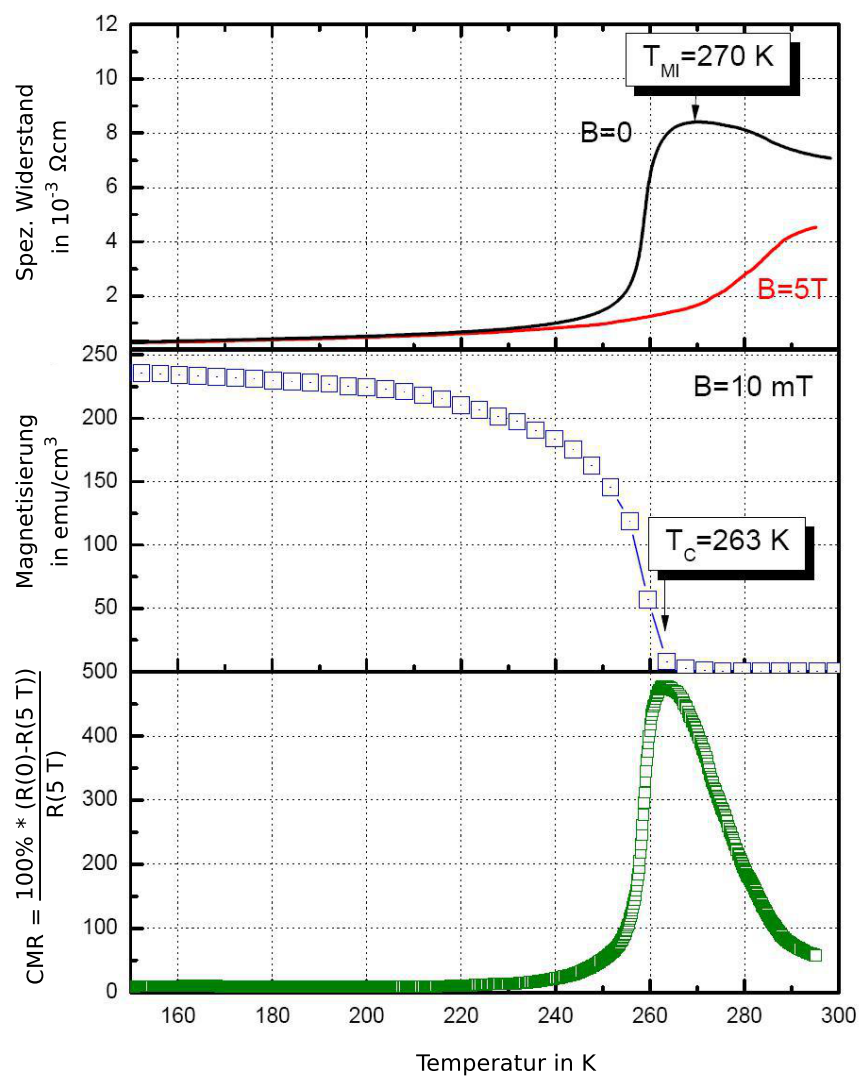

Abbildung 1.1: Metall-Isolator-Übergang und CMR-Effekt eines $\mathrm{La}_{0,75} \mathrm{Ca}_{0,25} \mathrm{MnO}_{3}$-Films. Spezifischer Widerstand (oben) und Magnetisierung (Mitte) zeigen einen sich etwa bei der Curie-Temperatur ereignenden Metall-Isolator-Übergang. Im Magnetfeld wird der Widerstand erheblich reduziert; das CMR-Verhältnis ist unten gezeigt. Aus [29]. 


\subsection{Kristallstruktur}

Diese Arbeit befasst sich mit Manganoxiden mit kolossalem Magnetowiderstandsverhalten. Die untersuchte Stoffklasse kristallisiert, wie schon die ersten Untersuchungen von Jonker und van Santen zeigten, in der sogenannten Perowskitstruktur. ${ }^{[12]}$ Der Begriff „Manganate“ bezeichnet im Rahmen dieser Abhandlung also stets Materialien vom stöchiometrischen Typ $\mathrm{AMnO}_{3}$.

Die in Abb. 1.2 dargestellte, idealisierterweise kubische Struktur setzt sich aus einem zentralen A-Platz-Ion im Mittelpunkt der Einheitszelle und $\mathrm{MnO}_{6}$-Oktaedern auf den Würfelecken zusammen. Der B-Platz ist also mit Manganionen besetzt, die Kantenmittelpunkte des Würfels mit Sauerstoffionen.

Physikalisch sind dabei insbesondere gemischtvalente Systeme von Interesse, welche der allgemeinen ionischen Summenformel $\mathrm{Re}_{1-\mathrm{x}}^{3+} \mathrm{Ae}_{\mathrm{x}}^{2+}\left(\mathrm{Mn}_{1-\mathrm{x}}^{3+} \mathrm{Mn}_{\mathrm{x}}^{4+}\right) \mathrm{O}_{3}$ folgen; mittels einer Verteilung von di- und trivalenten APlatz-Ionen erzwingt man das gleichzeitige Vorliegen von $\mathrm{Mn}^{4+}$ und $\mathrm{Mn}^{3+}$ im selben Mengenverhältnis. $\operatorname{Re}^{3+}$ bezeichnet dabei Seltene Erden wie $\mathrm{La}$, $\mathrm{Pr}$ und $\mathrm{Nd}, \mathrm{Ae}^{2+}$ Erdalkalimetalle (Ca, Sr und Ba).

Neben der direkten Valenzkontrolle der über jeweils sechs Sauerstoffbrücken koordinierten, für den elektrischen Transport maßgeblichen Mn-Ionen, kommt der Wahl der A-Platz-Ionen auch eine große strukturelle Bedeutung zu. Durch ihren vom kubischen

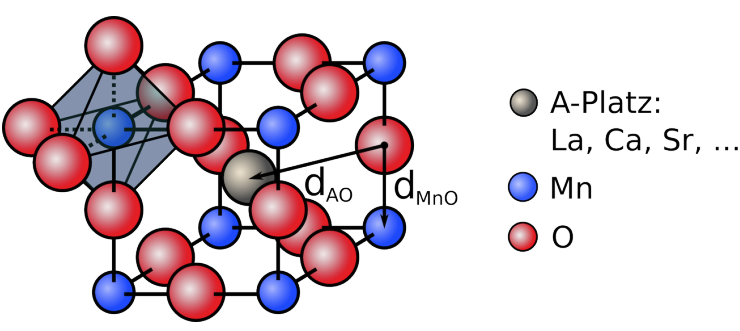

Abbildung 1.2: Struktur perowskitischer Manganate (schematisch). Das zentrale APlatz-Ion ist von kubisch angeordneten $\mathrm{MnO}_{6}$-Oktaedern umgeben. Die zur Bestimmung des Toleranzfaktors relevanten Ionenabstände sind hervorgehoben.

Idealfall abweichenden Ionenradius ergeben sich verzerrte, niedersymmetrische Strukturen. Ein nützliches Kriterium stellt dabei der Goldschmidtsche Toleranzfaktor dar:

$$
f=\frac{1}{\sqrt{2}} \frac{d_{\mathrm{AO}}}{d_{\mathrm{MnO}}} \approx \frac{1}{\sqrt{2}} \frac{<r_{\mathrm{A}}>+r_{\mathrm{O}}}{<r_{\mathrm{Mn}}>+r_{\mathrm{O}}} .
$$

Die Ionenabstände $d$ können anhand ihrer gemittelten Radien $r$ abgeschätzt werden. Im kubischen Fall läge ein Toleranzfaktor von $f=1$ vor, tatsächlich sind kleinere Werte realisiert. So stellt sich für $1>f>0,96$ eine noch als „pseudokubisch“ bezeichnete rhomboedrische $(\mathrm{R} \overline{3} \mathrm{c})$ Struktur ein. Für noch größere Abweichungen $f<0,96$ erhält man ein orthorhombisch verzerrtes Gitter (Pnma). ${ }^{[30]}$ In Abb. 1.3 sind beide Modifikationen des idealen Perowskitgitters skizziert.

Die Eigenschaften gemischtvalenter Manganatsysteme werden jedoch nur in begrenztem Umfang durch die obige Mittelung der Ionenradien erfasst. Tatsächlich spielen Unordnungsphänomene, Auswirkungen der stochastisch verteilten Ionenradien und der damit einhergehenden elastischen wie auch elektronischen Fluktuationen, nachweisbar eine bedeutende Rolle. ${ }^{[31]}$ Es können ferner sogar Kationenordnungen auftreten, sodass die Struktur durch eine größere ,Superzelle“ beschrieben werden muss, deren Bestandteile ihre elastischen Verspannungen und Deformationen untereinander kompensieren. ${ }^{[32]}$ 

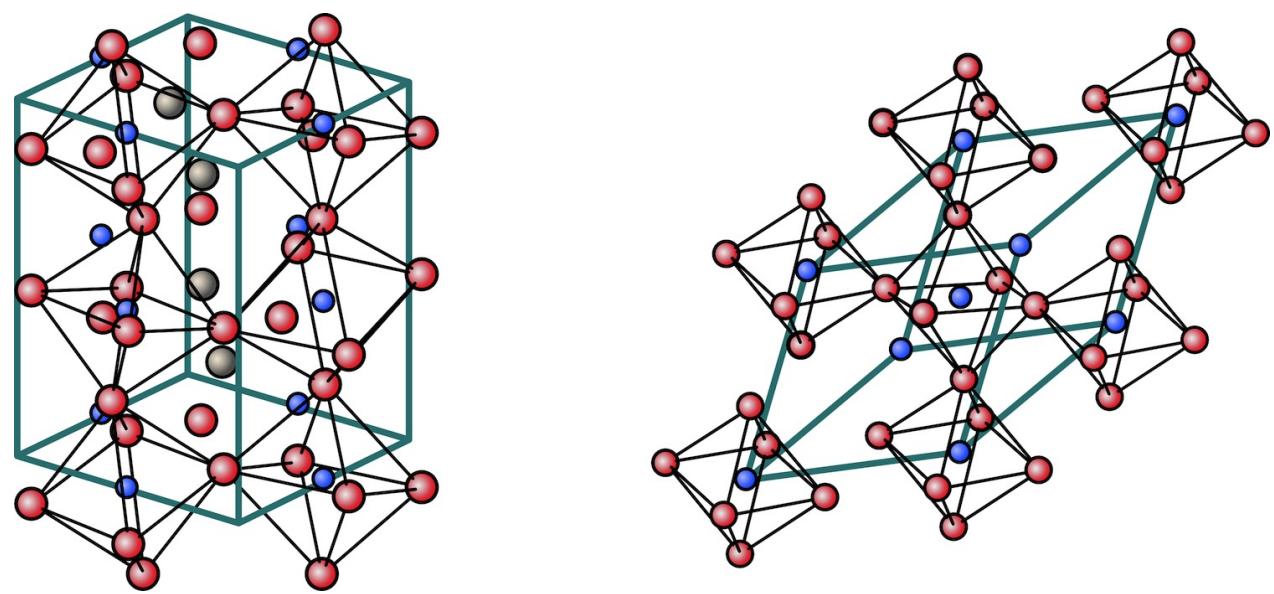

Abbildung 1.3: Modifikationen der idealen Perowskitstruktur. Die orthorhombische Struktur (links) ergibt sich für $f<0,96$, der rhomboedrische Fall für $1>f>0,96$ (rechts). Nach [30].

\subsection{Elektronische und magnetische Eigenschaften}

Im Folgenden werden die wesentlichen elektronischen Eigenschaften von Manganaten besprochen. Dabei wird zuerst die energetische Hierarchie der für Transporteigenschaften und magnetische Ordnungen relevanten Niveaus dargestellt. Die anschließende Beschreibung der wichtigsten Transportmechanismen ermöglicht ein qualitatives Verständnis des CMREffektes und der im nächsten Abschnitt diskutierten Phasendiagramme von Manganaten.

\subsubsection{Kristallfeldaufspaltung und Jahn-Teller-Effekt}

Die Physik der Manganate ist wesentlich durch die orbitale Struktur der Mn-Ionen und deren Hybridisierung mit den benachbarten $\mathrm{O}^{2-}$-Ionen bestimmt. Der Einfluss der unabgeschlossenen, aber sehr kernnahen, $4 f$-Schalen der A-Platz-Ionen ist nicht von Bedeutung. In diesem Abschnitt werden die wichtigsten Aspekte der elektronischen Struktur der Mn-Ionen skizziert; dabei folgen wir der Hierarchie von den vorherrschenden energetischen Einflüssen hin zu subtileren, aber dennoch weitreichenden Effekten.

Die Bevölkerung der 3d-Orbitale ist von der starken lokalen Coulombabstoßung, beziehungsweise der daraus resultierenden Hundschen Kopplung $J_{\mathrm{H}} \approx 2-3 \mathrm{eV}^{[34]}$ dominiert. Eine Doppeltbesetzung von Orbitalen wird also gemäß der Hundschen Regeln vermieden und eine Spinmaximierung begünstigt.

Während in einem freien Mn-Ion die fünf $3 d$-Orbitale entartet sind, erfahren sie im oktaedrischen Kristallfeld, das heißt im Coulomb-Potential der sie umgebenden $\mathrm{O}^{2-}$-Ionen, eine energetische Aufspaltung $\Delta_{\mathrm{CF}} \approx 1-2 \mathrm{eV}^{[21,34]}$.

Eine korrekte Herleitung erfordert eine aufwendige gruppentheoretische Behandlung, deren Essenz sich jedoch schon anhand der in Abb. 1.4 dargestellten Winkelanteile der Wellenfunktionen plausibel machen lässt: Die Keulen großer Aufenthaltswahrscheinlichkeit dreier Orbitale weisen in äquivalenter Form in Raumwinkelbereiche zwischen den Nachbarionen, sodass nur eine geringe Coulombabstoßung mit den dortigen Elektronen 

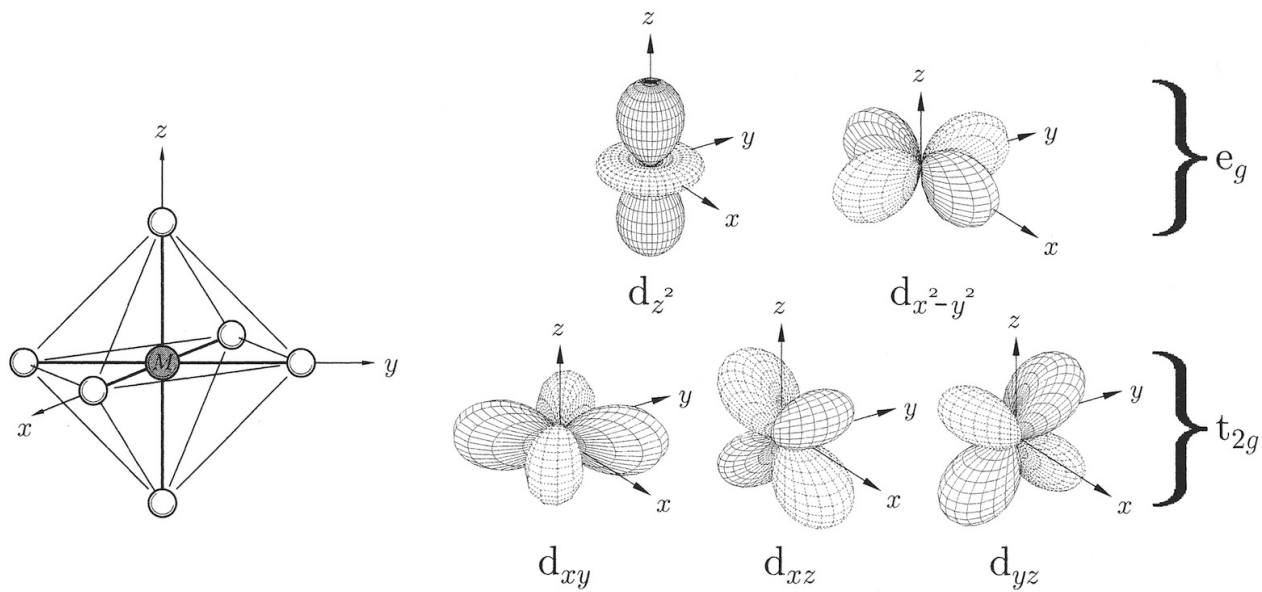

Abbildung 1.4: 3d-Orbitale des Mangans, aus [33]. Man beachte die Orientierung zu den benachbarten, auf den kartesischen Achsen lokalisierten Ionen (links). Für die $t_{2 g}$-Orbitale $d_{x y}, d_{y z}$ und $d_{z x}$ ergibt sich eine große Aufenthaltswahrscheinlichkeit im Raumwinkelbereich der nächsten Nachbarn, für die $e_{g}$-Zustände $d_{3 z^{2}-r^{2}}$ (bzw. $d_{z^{2}}$ ) und $d_{x^{2}-y^{2}}$ dagegen in Richtung der Zwischenräume.

vorliegt. Diese sogenannten $t_{2 g}$-Orbitale sind daher energetisch abgesenkt, während die Besetzung der verbleibenden beiden $e_{g}$-Orbitale Energie kostet, da sie gerade ihre größte Aufenthaltswahrscheinlichkeit in Richtung der Nachbarionen besitzen. Die energetische Aufspaltung ist dabei so beschaffen, dass sich die Anhebungen und Absenkungen im Falle einer vollständigen Besetzung mit zehn $d$-Elektronen genau kompensieren. Die $t_{2 g^{-}}$und $e_{g}$-Orbitale sind untereinander jeweils entartet.

(a)

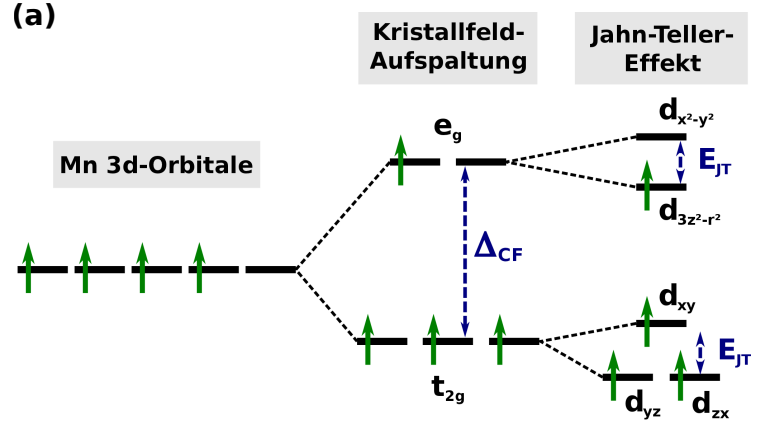

(b)

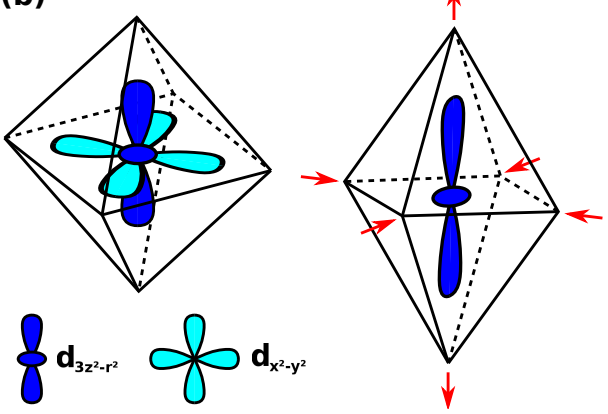

Abbildung 1.5: Kristallfeldaufspaltung und Jahn-Teller-Effekt. Die fünf entarteten $3 d-$

Orbitale des freien Ions spalten im oktaedrischen Kristallfeld in drei $t_{2 g^{-}}$und zwei $e_{g^{-}}$ Orbitale auf. $\mathrm{Mn}^{4+}$ ist ferner Jahn-Teller-aktiv: Eine Besetzung des $d_{3 z^{2}-r^{2}}$ ist gegenüber der des $d_{x^{2}-y^{2}}$ begünstigt (a). Diese energetische Absenkung liegt in der Elongation des $\mathrm{MnO}_{6}$-Oktaeders entlang der $z$-Achse begründet, wodurch ein geringerer Überlapp mit den umgebenden $\mathrm{O}_{2 p}$-Orbitalen entsteht (b). 
Die Entartung der $t_{2 g^{-}}$und $e_{g}$-Orbitale wird nun für bestimmte Besetzungen beziehungsweise Wertigkeiten, darunter $\mathrm{Mn}^{3+}$, ferner durch den Jahn-Teller-Effekt ${ }^{[35]}$ aufgehoben: Im Zuge einer Reduktion der in diesem Falle vollen oktaedrischen Symmetrie $O_{h}$ durch eine Elongation entlang einer Achse - willkürlich hier der $z$-Achse - gelingt eine Erniedrigung der Gesamtenergie. Diese Streckung eröffnet dem $d_{3 z^{2}-r^{2}}$-Orbital einen größeren Raumbereich, sodass wieder eine Verringerung des Überlapps mit den Nachbarn eine Energieerniedrigung bewirkt; die gleichzeitige Verkürzung der Bindungslängen in der $x$ - $y$-Ebene resultiert umgekehrt in einer erhöhten Energie des $d_{x^{2}-y^{2}}$-Zustandes. Wie in Abb. 1.5 dargestellt, erfahren die $t_{2 g}$-Orbitale eine ähnliche Aufspaltung, bei welcher das $d_{x y}$-Orbital energetisch ungünstiger wird. Die Jahn-Teller-Aufspaltung ist mit $E_{\mathrm{JT}} \approx 0,5-1,0 \mathrm{eV}$ relativ klein. ${ }^{[23]}$

Im Falle des $\mathrm{Mn}^{4+}$ sind nur die $t_{2 g}$-Orbitale mit jeweils einem Elektron besetzt. Unter Jahn-Teller-Verzerrung ergäbe sich daher kein energetischer Gewinn, weil die Änderungen einander kompensierten und zusätzlich Deformationsenergie aufgebracht werden müsste $\mathrm{Mn}^{4+}$ ist nicht „Jahn-Teller-aktiv“ und stabilisiert demzufolge die oktaedrische Symmetrie.

\subsubsection{Transportmechanismen und Magnetismus}

Die nachfolgenden Betrachtungen bilden die Basis für ein qualitatives Verständnis der elektrischen Leitfähigkeit und der beobachteten magnetischen Eigenschaften in Manganaten. Die Ausbildung magnetischer Ordnung geschieht dabei über Wechselwirkungen zwischen den $e_{g}$-Elektronen des Mangans. Die sich ergebende magnetische Ordnung erstreckt sich wegen der starken Hundschen Wechselwirkung jedoch auch auf die lokalisierten $t_{2 g}$-Elektronen. Wegen des großen räumlichen Abstandes sind vor allem indirekte, das heißt durch die Hybridisierung mit den Sauerstoffionen vermittelte Kopplungsmechanismen relevant.

\section{Superaustausch}

Zum Verständnis des antiferromagnetischen Grundzustandes gering dotierter Lanthanmanganate betrachten wir zunächst das Hubbard-Modell:

$$
\mathcal{H}=U \sum_{i} \boldsymbol{n}_{i \uparrow} \boldsymbol{n}_{i \downarrow}-t \sum_{\langle i, j\rangle, \sigma}\left(\boldsymbol{c}_{i \sigma}^{\dagger} \boldsymbol{c}_{j \sigma}+\boldsymbol{c}_{j \sigma}^{\dagger} \boldsymbol{c}_{i \sigma}\right) .
$$

Dabei stellen $\boldsymbol{c}^{\dagger}, \boldsymbol{c}$ und $\boldsymbol{n}$ die fermionischen Erzeugungs-, Vernichtungs- und Besetzungszahloperatoren sowie $U$ die lokale Coulombabstoßung dar. Die Summation $\langle i, j\rangle$ erstreckt sich über alle nächsten Nachbarn der durch $i$ und $j$ indizierten Gitterplätze. $t$ ist die „Hüpfamplitude“, welche dem Überlappintegral der beteiligten Wellenfunktionen entspricht. Der Transport erfolgt unter Erhaltung der Spinrichtung, dabei wird vereinfachend ein direkter Austausch, das heißt ohne explizit einbezogene Beiträge der Sauerstofforbitale behandelt.

Für halbe Füllung, also genau ein $e_{g}$-Elektron pro Mangan-Ion, entspricht diese Situation der des undotierten Lanthanmanganats $\mathrm{LaMnO}_{3}$. Die für Manganate große Coulombabstoßung $U \gg t$ unterbindet die Doppelbesetzung von Orbitalen. Ein kinetischer Energiegewinn kann über die in Abb. 1.6 veranschaulichten virtuellen Hüpfprozesse erfolgen. Entscheidend ist dabei die relative Spinorientierung der beteiligten $t_{2 g}$-Rumpfmomente: 

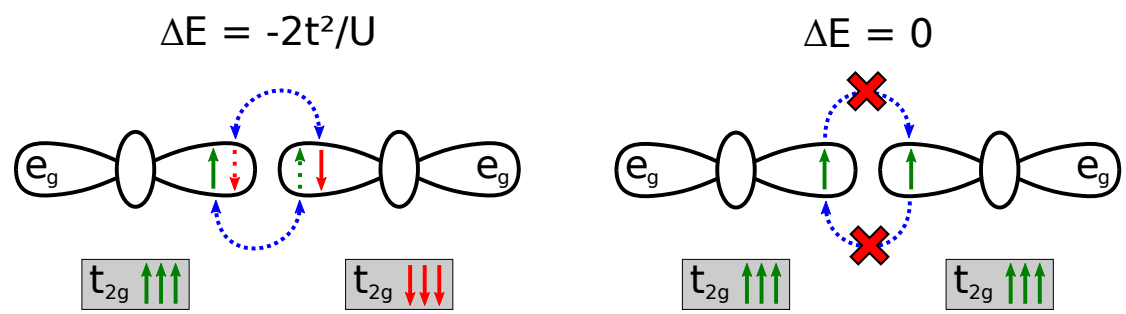

Abbildung 1.6: Superaustausch (schematisch). Während bei einer antiparallelen Anordnung der $t_{2 g}$-Spins ein kinetischer Energiegewinn über virtuelle Hüpfprozesse (links) möglich ist, wird dieser unter ferromagnetischer Ausrichtung durch das Pauli-Verbot unterbunden (rechts). Der Superaustauschmechanismus begünstigt daher die Ausbildung eines antiferromagnetisch geordneten Isolators.

- Im parallelen Fall sind jegliche virtuellen Hüpfprozesse aufgrund des Pauli-Prinzips verboten. Es erfolgt kein kinetischer Energiegewinn durch eine Abschwächung der Lokalisierung der Elektronen.

- Bei antiparalleler Ausrichtung können $e_{g}$-Elektronen auf benachbarten Plätzen in virtuellen Prozessen hin- und wieder zurückhüpfen. Diese Bewegung geht nach störungstheoretischer Behandlung zweiter Ordnung mit einer Verringerung der kinetischen Energie von $-\frac{2 \Delta t^{2}}{U}$ einher, ermöglicht aber keinen Nettotransport von Ladungsträgern. Man bezeichnet derartige Systeme als „Mott-Hubbard-Isolatoren“.[36]

Der nur unter entgegengesetzter Spinausrichtung erzielbare Energiegewinn durch virtuelles Hüpfen bewirkt daher eine antiferromagnetische Ordnung.

Das obige „Ausintegrieren“ der $\mathrm{O}_{2 p}$-Zustände ist strenggenommen nur dann zulässig, wenn ihre Einteilchenniveaus wie bei $\mathrm{LaMnO}_{3}$ gegenüber den Mn-3d-Zuständen um einen Betrag $E_{\mathrm{CT}} \gg U$ abgesenkt sind. Im Falle $E_{\mathrm{CT}} \ll U$ ergeben sich geringfügige Unterschiede und man spricht von einem „Ladungstransfer-Isolator“.[37,38] Grundsätzlich müssen auch weitere Faktoren wie die Hybridisierung mit den Sauerstofforbitalen und die sich ergebenden Änderungen für entartete $e_{g}$-Orbitale - in Abwesenheit etwa von Jahn-Teller-Verzerrungen - berücksichtigt werden. Im letzteren Falle kann insbesondere auch eine schwache ferromagnetische Wechselwirkung entstehen. ${ }^{[39]}$ Eine genauere Untersuchung dieser Feinheiten ergab die semiempirischen Goodenough-Kanamori-Anderson-Regeln, welche oftmals die Spinordnung korrekt vorhersagen können.

\section{Doppelaustausch}

Die Beschreibung dieses Transportmechanismus geht auf Clarence Zener ${ }^{[40,41]}$ zurück und wurde von Anderson \& Hasegawa ${ }^{[42]}$ und de Gennes ${ }^{[43]}$ erweitert. Das Doppelaustauschmodell bildet den Ausgangspunkt für ein qualitatives Verständnis des Ferromagnetismus in Manganaten.

Ursprünglich unterschied Zener zwischen lokalisierten $d$-Elektronen - in unserem Fall die $t_{2 g}$-Elektronen - und Leitungselektronen, hier den beweglichen $e_{g}$-Elektronen. 
Der Transport muss über die Hybridisierung mit den $\mathrm{O}_{2 p}$-Orbitalen erfolgen, welche jedoch bereits vollständig besetzt vorliegen. Der in Abb. 1.7 illustrierte Doppelaustauschmechanismus besteht nun im gleichzeitigen Hüpfen eines Elektrons vom $\mathrm{Mn}^{3+}$ in das Sauerstofforbital und eines der gleichen Spinorientierung von dort auf ein benachbartes $\mathrm{Mn}^{4+}$-Ion. Dieses Szenario beschreibt somit also auch einen tatsächlichen Ladungstransport.

Da auf jedem Ion durch die Hundsche Kopplung $J_{\mathrm{H}}$ eine parallele Ausrichtung bevorzugt wird, ergibt sich eine höhere Beweglichkeit bei parallel orientierten benachbarten $t_{2 g}$-Momenten, das heißt unter ferromagnetischer Ordnung. Die resultierende Spinpolarisierung von 100\% wurde durch Photoemission experimentell bestätigt. ${ }^{[2]}$

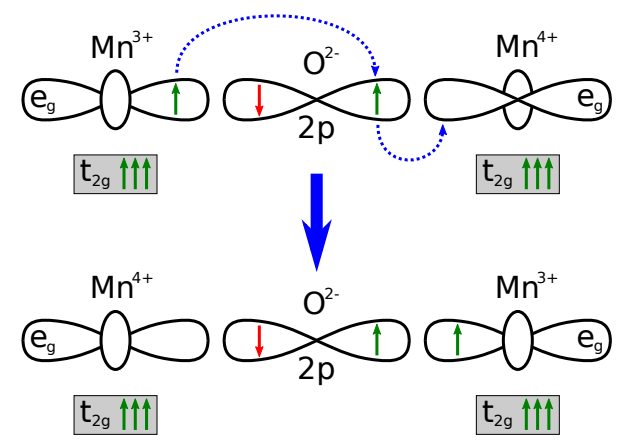

Üblicherweise wird der Beitrag der Sauerstofforbitale im Rahmen einer Tight-BindingBehandlung ,ausintegriert" und die darüber indirekt erfolgende Kopplung zweier benachbarter Mn-Ionen durch ein Hüpfmatrixelement $t_{\text {eff }}$ zusammengefasst. Für dieses zeigten Anderson und Hasegawa eine Abhängigkeit von der relativen Verkippung $\theta$ der beiden $t_{2 g}$-Rumpfspins: ${ }^{[42]}$

$$
t_{\mathrm{eff}}=t \cdot \cos \frac{\theta}{2} .
$$

Das Transferintegral $t$ ist im Tight-Binding-Modell direkt proportional zur Bandbreite und damit maßgeblich für den elektronischen Transport. Es hängt vom räumlichen Überlapp der Mangan- und Sauerstoffionen ab, welcher selbst nicht allein durch den Abstand, sondern vor allem auch von der Verkippung der benachbarten Sauerstoffoktaeder $\phi$ gemäß $t \propto \cos \phi$ bestimmt wird. ${ }^{[21]}$ Daher entsteht eine direkte Abhängigkeit der elektronischen Eigenschaften von dem Toleranzfaktor $f$, bzw. dem mittleren A-Ionenradius.

Das Doppelaustauschszenario ermöglicht somit eine qualitative Beschreibung des CMREffektes: Der Metall-Isolator-Übergang vollzieht sich in der Nähe der Curie-Temperatur, weil der kinetische Energiegewinn durch Doppelaustausch eine ferromagnetische Ordnung der $t_{2 g}$-Spins erfordert. Der Widerstand kann in der Nähe des Übergangs reduziert werden, wenn man durch Anlegen eines externen Magnetfeldes diese Ordnung forciert.

\subsection{Polaronen und Ladungsordnung}

Die oben vorgestellten Transportmechanismen erfassen zwar die Essenz des CMR-Effektes, offenbaren aber nicht allein in den Details des Metall-Isolator-Übergangs erhebliche Unzulänglichkeiten. Das Doppelaustauschszenario allein wird der phänomenologischen Viel- 
seitigkeit und dem Phasenreichtum der Manganate nicht gerecht und versagt bei der Beschreibung einer Reihe von experimentellen Resultaten. So erscheint beispielsweise das Vorliegen von Antiferromagnetismus gepaart mit metallischer Leitfähigkeit ${ }^{[44]}$ im Falle von $\mathrm{La}_{0,46} \mathrm{Sr}_{0,54} \mathrm{MnO}_{3}$ paradox. Dass ferner die durch den A-Ionenradius kontrollierbare Gitterverzerrung nicht linear in den Widerstand eingeht ${ }^{[45]}$ und die Übergangstemperatur von $\mathrm{La}_{0,8} \mathrm{Ca}_{0,2} \mathrm{MnO}_{3}$ einen starken Isotopeneffekt bei Substitution von ${ }_{8}^{16} \mathrm{O}$ durch ${ }_{8}^{18} \mathrm{O}$ zeigt, ${ }^{[46]}$ weist eindeutig auf einen starken Einfluss der Gittereigenschaften hin.

Millis et al. erkannten das Versagen des Doppelaustauschmodells für $\mathrm{La}_{1-\mathrm{x}} \mathrm{Sr}_{\mathrm{x}} \mathrm{MnO}_{3}$ (LSMO) und betonten die Bedeutung der Elektron-Phonon-Kopplung ${ }^{[47,48]}$ und quantisierten diese durch das dimensionslose Maß $\lambda=E_{\text {gitt }} / t_{\text {eff }}{ }^{[49]}$ Es wird dabei der kinetische Energiegewinn durch ein nach dem Doppelaustauschbild delokalisiertes Elektron, proportional zu $t_{\text {eff }}$, mit der Energie $E_{\text {gitt }}$ verglichen, welche ein lokalisiertes Elektron mit der es umgebenden Jahn-Teller-Verzerrung aufweist.

Es besteht daher eine Tendenz zur Selbstlokalisierung eines Ladungsträgers - das Elektron beziehungsweise Loch erzeugt eine lokale Gitterverzerrung, in deren „Käfig“ es verweilt; die effektive Masse der Ladungsträger wird erhöht. Der für Manganate etablierte Begriff des Polarons als Ladungsträger ist dabei allerdings nicht ganz unproblematisch. Eine Aufschlüsselung des genauen Charakters der Polaronen und ihrer Wechselwirkungen untereinander ist nämlich bis heute nicht gelungen. Verbreitet ist die Annahme einer Art von kleinen Holstein-Polaronen ${ }^{[50,51]}$ oder Jahn-Teller-Polaronen, also Elektronen, die eine lokale JahnTeller-Verzerrung ihrer Umgebung mit sich führen. ${ }^{a}$ Für hohe Temperaturen $T \gg T_{\mathrm{C}}$ gilt die polaronische Natur der Ladungsträger inzwischen als erwiesen, beispielsweise durch das typische, thermisch aktivierte Transportverhalten in $\mathrm{La}_{0,7} \mathrm{Ca}_{0,3} \mathrm{MnO}_{3}$ (LCMO). [53]

Darüberhinaus wurden mittels Röntgen${ }^{[54]}$ und Neutronenbeugung ${ }^{[55]}$ sogenannte „korrelierte Polaronen“ aufgefunden - kleine ladungsgeordnete Cluster. Von besonderer Stabilität ist dabei theoretischen Betrachtungen zufolge die sogenannte „CE-Phase“, ein Bandisolator. Diese in Abb. 1.8 veranschaulichte Orbitalordnung besteht aus in Zickzack-Ketten organisierten $d_{3 x^{2}-r^{2}}$ und $d_{3 y^{2}-r^{2}}$-Orbitalen, an den Ecken befinden sich jeweils $\mathrm{Mn}^{4+}$-Ionen. Das feldgetriebene Aufschmelzen anderer Polaronenordnungen in $\mathrm{Pr}_{1-\mathrm{x}} \mathrm{Ca}_{\mathrm{x}} \mathrm{MnO}_{3}$ (PCMO) wurde von Jooss et al. mit den kolossalen Widerstandsphänomenen in Verbindung gebracht. ${ }^{[56]}$

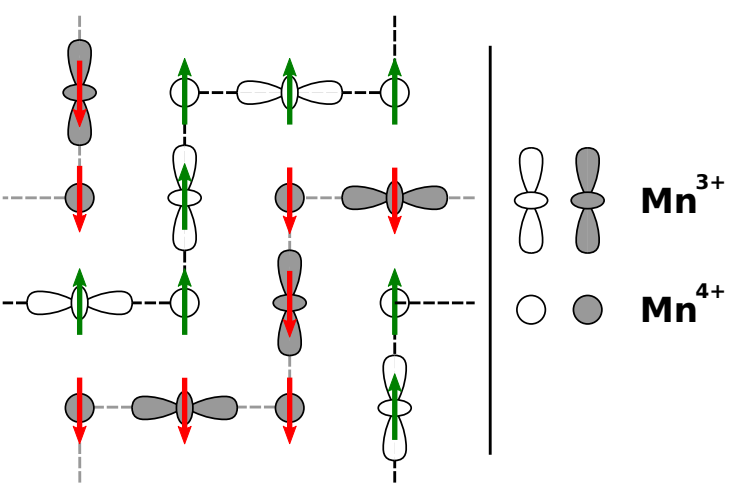

Abbildung 1.8: Orbital- und Spinordnung vom CE-Typ (schematisch).

Nicht nur in isolierenden, sondern auch im

ferromagnetischen Regime unterhalb von $T_{\mathrm{C}}$ gibt es Hinweise auf die Existenz korrelierter Polaronen, beispielsweise durch die Beobachtung von Streifenphasen in der Tunnelleitfähigkeit von Sudheendra et al. ${ }^{[57]}$

${ }^{\text {a}}$ Tatsächlich zeigen die Ergebnisse von Hartinger et al. jedoch Indizien für das Vorliegen sowohl kleiner Holstein- als auch ausgedehnterer Fröhlich-Polaronen in Manganaten. ${ }^{[52]}$ 
Ferner können Polaronen untereinander auch Bindungskräfte ausüben und so - nach der Hypothese von Alexandrov et al. - zum Beispiel durch Bildung von Bipolaronen ihre Beweglichkeit einbüßen. ${ }^{[58,59]}$ In diesem Bild findet in der Nähe des Metall-IsolatorÜbergangs durch Paarbildung eine drastische Verarmung an polaronischen Ladungsträgern statt, der sogenannte CCDC (charge carrier density collapse, Ladungsträgerdichtekollaps). Unterhalb von $T_{\mathrm{C}}$ werden die Bipolaronen durch die Austauschwechselwirkung wieder aufgebrochen und die Leitfähigkeit vergrößert.

Das sehr komplexe Wechselspiel der starken elektronischen Korrelationen und ihrer Kopplung an das Gitter wird gegenwärtig im Zuge neuer theoretischer Ansätze, allen voran der dynamischen Molekularfeldtheorie („Dynamic Mean Field Theory“, DMFT) intensiv studiert. Dabei konnten bereits beeindruckende Erfolge bei der Berechnung der Gittereigenschaften des kooperativ Jahn-Teller-verzerrten $\mathrm{LaMnO}_{3}$ und des verwandten, ebenfalls perowskitischen $\mathrm{KCuF}_{3}$ verzeichnet werden. ${ }^{[60,61]}$

\subsection{Phasendiagramme}

Die Phasendiagramme von Manganaten in Bezug auf den Gehalt ihrer Dotierung und die Spezies der Dopanten sind sehr komplex. Wir reduzieren diesen Detailreichtum und wenden uns nur den wesentlichen Charakteristika eines prototypischen Manganates, hier $\mathrm{La}_{1-\mathrm{x}} \mathrm{Ca}_{\mathrm{x}} \mathrm{MnO}_{3}$ (LCMO), zu. Es ist eines der am intensivsten untersuchten Manganate überhaupt; auch ein Großteil der in dieser Arbeit vorgestellten Ergebnisse bezieht sich auf LCMO. Das Phasendiagramm des ebenfalls untersuchten $\mathrm{La}_{1-\mathrm{x}} \mathrm{Sr}_{\mathrm{x}} \mathrm{MnO}_{3}$ (LSMO) ist zumindest auf der lochdotierten Seite $x<0,5$ qualitativ sehr ähnlich ${ }^{[62,63]}$, weist aber deutlich höhere Übergangstemperaturen auf.

LCMO ist im Sinne der von Dagotto eingeführten Klassifizierung ${ }^{[21,25]}$ von mittlerer Bandbreite, da der kinetische Energiegewinn im Doppelaustauschszenario und die Energie einer den Ladungsträger lokalisierenden Gitterverzerrung von ähnlicher Größe sind. Es kann deshalb je nach Dotierung sowohl einen ferromagnetischen als auch einen ladungsgeordnetisolierenden Grundzustand haben.

Abb. 1.9 zeigt das vollständige Phasendiagramm nach Cheong \& Hwang. ${ }^{64]}$ Demnach liegt bei $x=0$, also undotiertem Lanthanmanganat $\mathrm{LaMnO}_{3}$, in welchem ausschließlich Superaustausch möglich ist, verständlicherweise eine antiferromagnetische Spinordnung vor. Diese ist vom A-Typ, also von der Form sich entlang einer Kristallachse abwechselnder, ferromagnetisch geordneter Lagen entgegengesetzter Ausrichtung.

Durch sukzessive Erhöhung des Calciumanteils wird die Bedeutung des ferromagnetischen Doppelaustausches größer. Das aus dieser Konkurrenz mit dem Superaustausch entstehende, komplizierte Verhalten für $0<x<0,17$ enthält möglicherweise verkantete antiferromagnetische $(\mathrm{CAF}){ }^{\mathrm{b}}$ ferromagnetisch isolierende (FI) und ladungsgeordnete (charge-ordered, $\mathrm{CO}$ ) Regime.

Für größere Dotierungen vermag der Doppelaustausch im Grundzustand ferromagnetische Ordnung zu erzwingen; demzufolge erhält man hier ein spinpolarisiertes Metall. Die

\footnotetext{
${ }^{\mathrm{b}}$ Die genaue Beschaffenheit der ursprünglich von de Gennes ${ }^{[43]}$ vorgeschlagenen CAF-Phase ist bis dato ungeklärt; es wird unter anderem ein möglicher phasenseparierter Grundzustand von AF- und FMAnteilen erwogen. ${ }^{[26]}$
} 


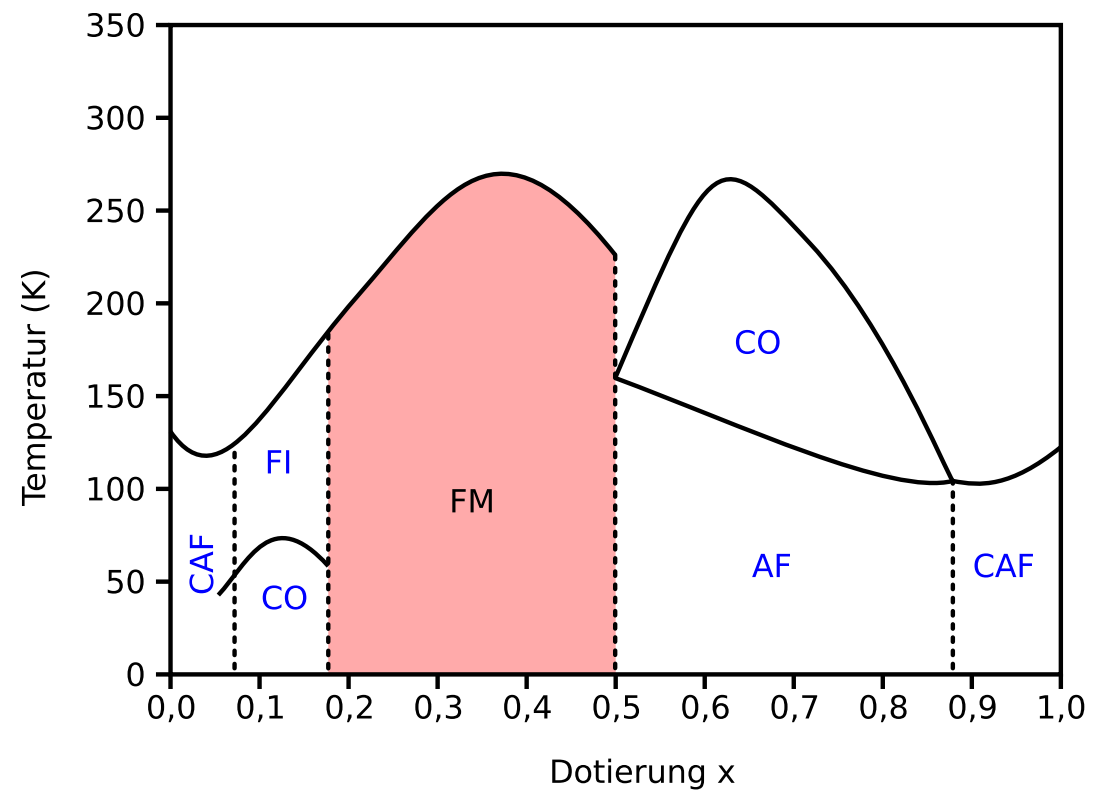

Abbildung 1.9: Phasendiagramm von $\mathrm{La}_{1-\mathrm{x}} \mathrm{Ca}_{\mathrm{x}} \mathrm{MnO}_{3}$, nach [64].

Unzulänglichkeiten dieser einfachen Beschreibung durch den Doppelaustausch offenbaren sich für mehr als halbdotiertes LCMO, wo eine antiferromagnetische, ladungs- und sogar orbitalgeordnete Phase auftritt. Im gemischtvalenten Regime ist dabei der Fall $x \approx 0,5$ von besonderem Interesse; hier ist die Ausbildung der oben dargestellten CE-Phase beobachtet worden. Für größere Dotierungen findet ein Übergang zu antiferromagnetischer Ordnung vom C-Typ statt.

Bei sehr hohen Lochdichten, $x>7 / 8$, befindet sich wieder ein CAF-Regime von nicht gänzlich verstandener Form. Für $x=1$, also im vollständig lochdotierten Falle, kann schließlich überhaupt kein Doppelaustausch mehr stattfinden, weil die $e_{g}$-Orbitale gänzlich entvölkert sind. Die direkte Austauschkopplung zwischen den Mn-Ionen bewirkt wieder eine antiferromagnetische Ordnung, diesmal vom G-Typ - abwechselnder Spinorientierung entlang aller drei Kristallrichtungen.

\subsection{Phasenseparation und Perkolation}

In diesem Abschnitt wenden wir uns mit der Phasenseparation einem Phänomen zu, welches die elektrischen Eigenschaften von Manganaten entscheidend beeinflusst. Die theoretischen Konzepte und Simulationsverfahren sind komplex und können hier nur in einem kurzen Überblick dargestellt werden. Für eine umfassende Einführung ist auf die Monographie von Dagotto zu verweisen. ${ }^{[21]}$ Im Rahmen der vorliegenden Arbeit durchgeführte Simulationen sind nicht der Schwerpunkt dieser Arbeit und werden deshalb im Anhang behandelt. Dort werden auch die verwendeten Modelle und numerischen Details näher ausgeführt.

Das unter 1.2.2 beschriebene Doppelaustauschbild bietet einen intuitiven Zugang zur Erklärung eines ferromagnetisch-metallischen Grundzustandes. Seine Anwendbarkeit ist jedoch bestenfalls auf Manganate großer Bandbreite beschränkt; es versagt spätestens bei 
der quantitativen Beschreibung des scharfen Metall-Isolator-Überganges und des CMREffektes für, beispielsweise, $\left(\mathrm{La}_{1-\mathrm{y}} \mathrm{Pr}_{\mathrm{y}}\right)_{1-\mathrm{x}} \mathrm{Ca}_{\mathrm{x}} \mathrm{MnO}_{3}$ (LPCMO). Man geht davon aus, dass diese Unzulänglichkeit in einer Koexistenz verschiedener Phasen begründet liegt. ${ }^{[21,25]}$

In Manganaten kann sich eine solche Phasenseparation auf sehr verschiedenen Längenskalen abspielen. So gibt es einerseits zahlreiche experimentelle Nachweise für die zuvor erwähnten ladungsgeordnet-isolierenden CE-Nanocluster, welche in eine metallische Umgebung eingebettet sind. Die CE-Cluster unterscheiden sich vom leitfähigen Hintergrund auch durch eine geringere Elektronendichte, es liegt daher durch die Phasenseparation auch eine Ladungstrennung vor. Die langreichweitige Coulombwechselwirkung verhindert deshalb eine Ausdehnung über die Nanometerskala hinaus. ${ }^{c}$ Es gibt jedoch trotzdem auch eine Vielzahl von experimentellen Hinweisen auf die Existenz einer Phasenseparation bis in den Mikrometerbereich. Wegweisend waren hier die elektronenmikroskopischen Untersuchungen von LPCMO-Einkristallen durch Uehara et al., die eine Phasenseparation auf einer im Submikrometerregime angesiedelten Längenskala zeigten. ${ }^{[65]}$ Darüberhinaus gibt es auch rastertunnelspektroskopische Belege, zum Beispiel von Fäth et al. und Becker et al., für ein solches Verhalten. ${ }^{[66,67]}$

Die Suche nach der Ursache derartig ausgedehnter Inhomogenitäten ist nicht einfach, da ihre Stabilität ja nur bei gleicher Elektronendichte vorstellbar ist. Moreo et al. untersuchten dazu die für Manganate etablierten Ein- und Zweiorbitalmodelle in MonteCarlo-Simulationen. ${ }^{[68]}$ Das einfachere Einorbitalmodell wird durch den folgenden HamiltonOperator beschrieben:

$$
\mathcal{H}=-t \sum_{\langle i, j\rangle, \sigma}\left(\boldsymbol{c}_{i \sigma}^{\dagger} \boldsymbol{c}_{j \sigma}+\boldsymbol{c}_{j \sigma}^{\dagger} \boldsymbol{c}_{i \sigma}\right)-J_{\mathrm{H}} \sum_{i, \alpha, \beta} \mathbf{s}_{i} \cdot \mathbf{S}_{i}+J_{\mathrm{AF}} \sum_{\langle i, j\rangle} \mathbf{S}_{i} \cdot \mathbf{S}_{j}
$$

Hierbei werden die $e_{\mathrm{g}}$-Elektronen am Gitterplatz $i$ und mit Spin $\sigma$ durch die Erzeugungsund Vernichtungsoperatoren $\boldsymbol{c}_{i \sigma}^{\dagger}$ beziehungsweise $\boldsymbol{c}_{i \sigma}$ beschrieben; der Spinoperator s ist über die Hundsche Wechselwirkung $J_{\mathrm{H}}$ an die Rumpfspins $\mathbf{S}$ gekoppelt. Diese erfahren eine antiferromagnetische Kopplung $J_{\mathrm{AF}}$ mit ihren nächsten Nachbarn; die Kopplung der $e_{\mathrm{g}}$-Orbitale wird durch $t$ vermittelt. Dieses Modellsystem zeigt - ohne Unordnung - einen Phasenübergang erster Ordnung in Bezug auf $J_{\mathrm{AF}} / t$ zwischen der durch $J_{\mathrm{AF}}$ begünstigten antiferromagnetischen Ordnung und der durch den Doppelaustausch via $t$ vermittelten ferromagnetischen Ausrichtung.

Moreo et al. trugen nun zusätzlich der intrinsischen Unordnung des Systems Rechnung, welche beispielsweise durch die aus der dotierungsbedingten Inhomogenität ${ }^{\mathrm{d}}$ resultierende Variation der Bindungswinkel zustande kommt. Dazu wählten sie keine konstanten Kopplungsparameter mehr, sondern ergänzten einen zufällig verteilten Beitrag zu den Hüpfamplituden $t$ und den antiferromagnetischen Kopplungen $J_{\mathrm{AF}}$ benachbarter Gitterplätze. ${ }^{[68]}$

\footnotetext{
${ }^{\mathrm{c}}$ Der thermodynamische Phasenbegriff, welcher auf der Längenskala des betreffenden Bereiches näherungsweise homogene physikalische Eigenschaften voraussetzt, ist aufgrund der oftmals nur sehr kleinen räumlichen Abmessungen in Manganaten problematisch. Wir machen hier dennoch von diesem für die Beschreibung von Manganaten üblichen Terminus Gebrauch.

${ }^{\mathrm{d}}$ Es sei darauf hingewiesen, dass hierunter keine chemische Inhomogenität im Sinne einer Entmischung innerhalb der Probe zu verstehen ist, sondern die zufällige Besetzung der A-Plätze mit Lanthan und den jeweiligen Dotierungen.
} 
Für eine sehr starke Unordnung, das heißt große zufällige Ergänzungen zu den Kopplungskonstanten, ergibt sich stattdessen eine feine Phasenseparation auf der Längenskala der Gitterabstände. Ist die Unordnung hingegen nur schwach ausgeprägt, so konkurrieren die lokalen Effekte mit dem zusätzlichen Energiebeitrag, der für eine Grenzfläche aufzubringen ist. Es stellt sich daher ein zwar phasenseparierter Kompromiss ein, der aber die Ausbildung einer großen Anzahl an Grenzflächen meidet: Es entstehen Domänen, die eine viel größere Ausdehnung aufweisen können als die Längenskala der ursprünglichen Unordnung selbst.

Wenn gleichzeitig metallische und isolierende Bereiche innerhalb einer Probe bestehen, wie es auch die Simulationen am realistischeren Zweiorbitalmodell nahelegen, so kann das Transportverhalten von sogenannten „Perkolationseffekten“ geprägt werden. Das bedeutet, dass der makroskopische Widerstand eines Systems entscheidend davon abhängt, ob ein leitfähiges Filament aus metallischen Domänen die Elektroden kurzschließt. Kleine Änderungen des metallischen Volumenanteils können so den stromführenden Pfad schließen oder unterbrechen und sich damit in drastischen Widerstandsänderungen äußern (vergleiche Abb. 1.10 (b)).

Bei der numerischen Behandlung von Perkolationsphänomenen führt man eine Diskretisierung durch das Aufstellen von Widerstandsnetzwerken durch. Bei hinreichend großen Netzwerken, die eine belastbare Simulation der perkolativen Prozesse gewährleisten, ist es jedoch nicht möglich, die für Manganate realistischen mikroskopischen Modelle zu lösen - der Rechenaufwand wäre nicht zu bewältigen. Man beschränkt sich daher auf einfachere Modellsysteme, die eine für das jeweilige Problem als realistisch angenommene Phasenkonfiguration zeigen, und überträgt sie auf ein Widerstandsnetzwerk.

(a) Widerstandsnetzwerk

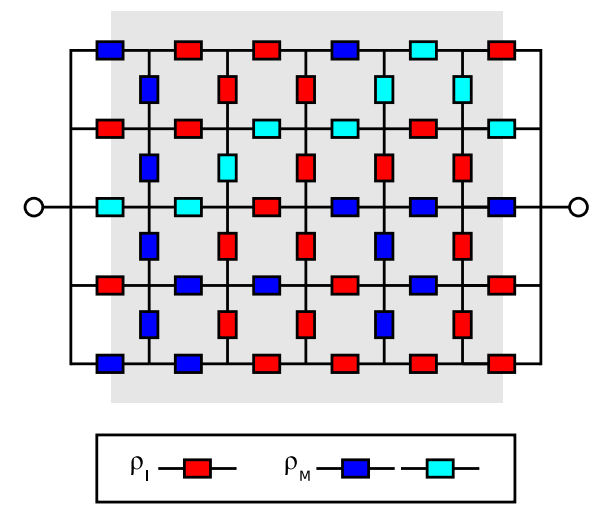

(b) Perkolativer Widerstandsverlauf

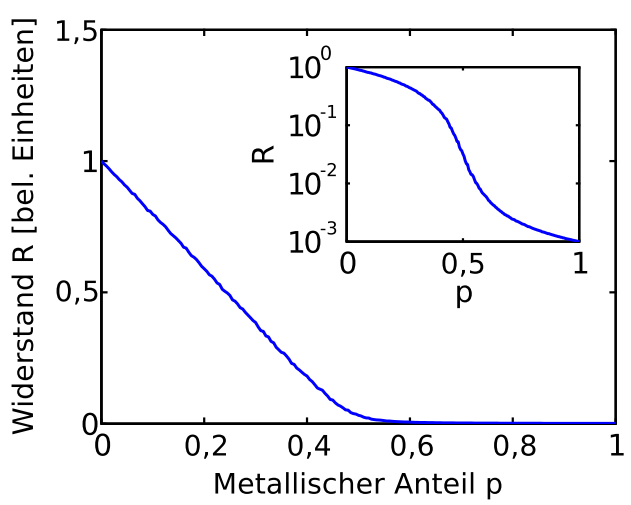

Abbildung 1.10: Zufälliges Widerstandsnetzwerk (a) mit perkolativem Widerstandsverhalten (b). Die Knoten sind jeweils mit der Wahrscheinlichkeit über einen blau dargestellten metallischen Widerstand $\rho_{\mathrm{M}}$ oder einen Isolator $\rho_{\mathrm{I}}$ (rot) verbunden. Der sich hier bei $p=0,5$ ausbildende, hellblau hervorgehobene Perkolationspfad verbindet den linken und rechten Kontakt. Der für ein $100 \times 100$-Netzwerk mit $\rho_{\mathrm{I}} / \rho_{\mathrm{M}}=1000$ simulierte Widerstandsverlauf ändert sich an der Perkolationsschwelle drastisch; eine logarithmische Auftragung wurde ergänzt. 
Als Beispiel ist in Abb. 1.10 (a) ein zweidimensionales Zufallsnetzwerk skizziert, wie es auch in den Arbeiten von Mayr et al. Verwendung fand. ${ }^{[10]}$ Zwei benachbarte Knotenpunkte werden hier zufällig mit einer Wahrscheinlichkeit $p$ mit einem kleinen metallischen Widerstand $\rho_{\mathrm{M}}$ überbrückt. Die restlichen Knotenpunkte werden mit einem sehr viel größeren, isolierenden Widerstand $\rho_{\mathrm{I}}$ oder, im einfachsten Falle, überhaupt nicht verbunden. Die Möglichkeit eines Stromflusses von der linken zur rechten Kante des Quadrates hängt jetzt davon ab, ob mindestens ein durchgehend leitender Pfad zwischen den beiden Elektroden existiert. In zwei Dimensionen liegt die dafür nötige minimale Besetzungswahrscheinlichkeit, die sogenannte „Perkolationsschwelle“, bei $p_{\mathrm{krit}}^{2 \mathrm{D}}=0,5$; in drei Dimensionen erhält man $p_{\text {krit. }}^{3 \mathrm{D}}=0,25$. $^{\mathrm{e}}$

In diesem Bild werden scharfe Übergänge durch das abrupte Kurzschließen oder Unterbrechen eines Perkolationspfades aufgrund einer geringfügigen Verschiebung der metallischen und isolierenden Phasengewichtung erklärbar. Man kann das Widerstandsverhalten so effektiv auf die in Abb. 1.11 gezeigten Beiträge reduzieren: Bei tiefen Temperaturen überwiegt der perkolative Transport durch die metallischen Filamente mit dem Widerstand $R_{\mathrm{M}}^{\mathrm{per}}(T)$. Für hohe Temperaturen ist der Widerstand der isolierenden Phase $R_{\mathrm{I}}(T)$ geringer als der der metallischen Bereiche, und der Strom fließt hauptsächlich durch den Isolator. Dazwischen stellt sich ein Maximum im Widerstand ein.

Für eine realistischere Beschreibung muss neben der Temperaturabhängigkeit der Widerstandsanteile auch eine Veränderung des metallischen Anteils in Abhängigkeit von Temperatur und Magnetfeld berücksichtigt werden. Auf diese Weise lassen sich schließlich die scharfen Metall-Isolator-Übergänge und auch die Stärke

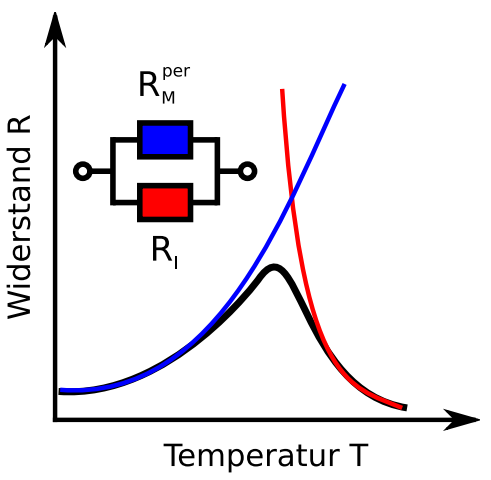

Abbildung 1.11: Effektives Zweiwiderstandsmodell nach Mayr. ${ }^{[10]}$ Für niedrige Temperaturen dominiert der perkolative Transport durch metallische Bereiche, für hohe der Strom durch die isolierenden Regionen. des CMR-Effektes begreifen: In der Nähe der CurieTemperatur besteht eine empfindliche energetische Balance der beiden konkurrierenden Phasen - das System ist nahe der Perkolationsschwelle. Beim Anlegen eines Magnetfeldes genügt schon eine kleine Vergrößerung des ferromagnetisch-metallischen Volumenanteils um drastische Perkolationseffekte im Widerstand hervorzurufen.

Beim Studium derartiger Einflüsse erwies sich das zweidimensionale Ising-Modell mit lokalem zufälligem Magnetfeld (random field ising model, RFIM - siehe [69]) als wegweisend. Es ist zwar für eine mikroskopische Beschreibung der Manganate an sich ungeeignet, generiert aber - je nach Unordnung auf unterschiedlicher Längenskala - eine Phasenseparation zwischen Bereichen mit auf- und abwärts orientiertem Spin. ${ }^{[68]}$ Dieses Modell besteht aus einem herkömmlichen Ising-Modell mit einer Kopplung $J$ der $z$-Komponenten $S_{i}^{z}$

\footnotetext{
${ }^{\mathrm{e}}$ Durch die begrenzte, endliche Anzahl von Knoten in $x$ - und $y$-Richtung besteht in den numerischen Simulationen die Möglichkeit, trotz $p=p_{\text {krit. }}$ unter zufälliger Besetzung der Widerstände keinen oder auch mehrere Perkolationspfade zu erhalten. Derartigen numerischen Effekten kann man durch Mittelung über mehrere zufällige Konfigurationen begegnen.
} 
benachbarter Spins; die Unordnung wird dem System hier durch ein lokales zufälliges Magnetfeld $h_{i}$ aufgeprägt:

$$
\mathcal{H}=-J \sum_{\langle i, j\rangle} S_{i}^{z} S_{j}^{z}-\sum_{i} h_{i} S_{i}^{z} \quad \text { mit } \quad S_{i}^{z} \in\{-1,+1\} .
$$

Die Domänenkonfiguration und damit das Perkolationsverhalten ist jetzt auch von der Temperatur abhängig - mit steigender Temperatur wird nämlich an Engstellen der Perkolationspfade die Spinordnung geschwächt. Diese paramagnetischen Regionen unterbrechen so schließlich die leitfähigen Filamente. Ein ähnliches Modell zur Untersuchung der subtilen Konkurrenz verschiedener geordneter Phasen wurde von Burgy et al. vorgestellt. [11]

Die in diesem Abschnitt erläuterten Konzepte machen deutlich, wie schwer der Zugang zum Transportverhalten der Manganate ist. Ein gemessener Temperaturverlauf des Widerstands kann nur bei gleichzeitig vorliegender Kenntnis über die Phasenseparation - auf dem ganzen untersuchten Temperaturintervall, also unter sehr großem experimentellem Aufwand - und durch Simulationsrechnungen komplexer Modelle in einem konsistenten Bild erklärt werden.

\subsection{Manganatoberflächen}

Während über die strukturellen und elektronischen Volumeneigenschaften von Manganaten schon vieles bekannt ist und eher das komplizierte Wechselspiel verschiedener Ordnungen untereinander und mit externen Feldern Gegenstand aktueller Bemühungen ist, so ist schon allein die Frage der genauen Beschaffenheit von vielen Manganatober- und -grenzflächen bis dato ungeklärt. Die experimentelle Strukturuntersuchung von Manganatoberflächen erweist sich als äußerst schwierig: Rastertunnelmikroskopie - üblicherweise die Methode der Wahl für hochauflösende laterale Messungen - ist im Falle von Manganaten meistens äußerst kompliziert.

Wie im vorigen Abschnitt bereits erwähnt, zeigen einige Ergebnisse eine auf Phasenseparation hinweisende Variation der Tunnelleitfähigkeit. ${ }^{[66,67]}$ Es berichteten bisher aber nur sehr wenige Veröffentlichungen von tatsächlich atomarer Auflösung an Manganaten, dabei zumeist für eher ,exotische“ Systeme. Bemerkenswert ist auf jeden Fall die direkte Beobachtung einer Phasenseparation in ladungsgeordnete und -ungeordnete Oberflächenbereiche an $\mathrm{Bi}_{1-\mathrm{x}} \mathrm{Ca}_{\mathrm{x}} \mathrm{MnO}_{3}$ durch Renner et al. ${ }^{[70]}$ Einhergehend mit einer strukturellen Umordnung wurde dabei eine Änderung des lokalen Transportverhaltens festgestellt. Rößler et al. ist ferner die Abbildung von nanoskaligen Streifenphasen gelungen; sie fanden im Zusammenhang damit außerdem Indizien für das Auftreten von Oberflächenrekonstruktionen. ${ }^{[1]}$ Ähnliche Ergebnisse streifenartiger Modulationen der elektronischen Zustandsdichte wurden von Sudheendra et al. erhalten. ${ }^{[57]}$

Neben den rastertunnelmikroskopischen direkten Abbildungen der Phasenseparation, welche ja für viele Manganate auch im Volumen als charakteristisch anzusehen ist, gibt es auch Ergebnisse, wonach die Grenz- und Oberflächen neuartige, einzigartige Eigenschaften besitzen können. Viele Manganatsysteme zeigen nachweislich eine Störung der ferromagnetischen Ordnung in der Nähe von Grenz- und Oberflächen. Diese Schwächung des Doppelaustausches, eventuell sogar bis hin zur Ausbildung antiferromagnetischer Strukturen, geht mit einer starken Reduktion der Leitfähigkeit einher. Experimentell machen 
sich derartige Abweichungen, man spricht von inaktiven oder "toten“ Lagen (Dead Layers), vor allem bei einer Verringerung der Schichtdicke bemerkbar - sehr dünne Filme verlieren ihre ferromagnetisch-metallischen Eigenschaften. ${ }^{[2,73]}$ Dass sich die kritischen Dicken für die Beobachtung einer ferromagnetischen Hysterese und für das Vorliegen metallischer Leitfähigkeit mit einem Metall-Isolatorübergang dabei eindeutig unterscheiden, kann unter der Annahme einer Phasenseparation an der Oberfläche - also in Einklang mit den rastertunnelmikroskopischen Ergebnissen - verstanden werden.

Angesichts der Tatsache, dass bereits die Volumeneigenschaften von einer empfindlichen Balance elektronischer, magnetischer und elastischer Effekte geprägt sind, ist es eigentlich wenig verwunderlich, dass eine derart massive Störung wie der Symmetriebruch an einer Grenz- oder Oberfläche sich dort auch in veränderten elektrischen, magnetischen und strukturellen Eigenschaften bemerkbar machen kann. Theoretische Arbeiten von Calderón et al. zufolge lässt sich durch den Symmetriebruch an Grenz- beziehungsweise Oberflächen von Manganaten eine deutliche Schwächung des Doppelaustausches erwarten, welche in einer abweichenden orbitalen Konfiguration in den Grenzlagen resultiert. ${ }^{[74]}$ Ebenso wie die Jahn-Teller-Aufspaltung der $e_{g}$-Orbitale kann auch der Bruch der kubischen Symmetrie nahe der Oberfläche eine Schwächung des Doppelaustausches bewirken. Ist die oberste Lage sauerstoffdefizitär, so kann die entstehende Aufspaltung demnach sogar die Größe typischer Jahn-Teller-Effekte in schwachdotierten Manganaten übertreffen. Die direkte antiferromagnetische Kopplung benachbarter $t_{2 g}$-Rumpfspins kann dann überwiegen und nicht nur ein isolierendes Verhalten der Oberfläche hervorrufen, sondern auch einen Ladungstransfer verursachen - ein Oberflächendipol entsteht. Nach Calderón et al. kann das alleinige Vorliegen einer solchen Oberfläche für optimal dotierte Systeme in einem Widerstandsanstieg von mehr als einer Größenordnung resultieren. Durch Messungen des linearen Röntgendichroismus für verschiedene Schichtdicken wiesen Tebano et al. Orbitalordnungen in der Nähe von Oberflächen nach, wonach der $d_{3 z^{2}-r^{2}}$-Typ übereinstimmend mit der Theorie energetisch begünstigt ist. ${ }^{[75]}$

Brey zeigte außerdem, dass die Grenzfläche zwischen einem halbmetallischen Manganat und einem Isolator auf ähnliche Weise zu einer Schwächung des Doppelaustausches und der Ausbildung antiferromagnetischer Instabilitäten neigen sollte und schlug vor, diese Tendenzen durch wenige zusätzliche Lagen undotierten Manganates an der Grenzfläche zu unterdrücken. ${ }^{[76]}$ Betont wurde dabei auch der starke Einfluss des Verspannungszustandes der Grenzfläche, also die Kopplung an strukturelle Eigenschaften. Gehrke et al. zeigten für Manganat-Titanat-Schichtsysteme tatsächlich, dass eine derartige Modifikation der Grenzflächen sehr wirksam den Ferromagnetismus und die Leitfähigkeit wiederherstellt und die elastische Kopplung außerdem auch tief in den Schichten noch eine strukturelle Änderung induzieren kann. ${ }^{[77,78]}$ 


\section{Elektrisches Widerstandsschalten}

Im vorigen Kapitel wurde bereits der thermisch getriebene Metall-Isolator-Übergang und der damit einhergehende Kolossale Magnetowiderstandseffekt von Manganaten behandelt. Neben diesen zumindest ansatzweise verstandenen Phänomenen gibt es noch weitere Widerstandseffekte an Manganaten, deren Beschreibung auch auf fundamentaler Ebene noch Gegenstand heftiger Diskussion ist. Sie umfassen Leitfähigkeitsänderungen, die durch so verschiedene Stimuli wie die mechanische Verspannung, optische Anregungen oder elektrische Ströme und Felder hervorgerufen werden können. Gegenstand dieser Arbeit sind elektrisch getriebene Widerstandseffekte.

Vom praseodymdotierten Lanthanmanganat ist eine als CER („Colossal Elektroresistance“) bezeichnete Widerstandsänderung in starken elektrischen Feldern beziehungsweise Strömen bekannt, welche bis zu 4 Größenordnungen umfassen kann. ${ }^{[3]}$ Dieser Effekt hat einen Volumencharakter und erfordert die Existenz einer isolierenden, ladungsgeordneten Phase, ${ }^{\text {a }}$ welche im Feld aufschmilzt.

Darüberhinaus sind auch oberhalb der Ladungsordnungstemperatur ausgeprägte, elektrisch verursachte resistive Effekte beobachtet worden, welche nach dem derzeitigen Kenntnisstand eher als Ober- beziehungsweise Grenzflächenphänomene aufgefasst werden müssen. Die induzierten Widerstandsänderungen können dabei auch ohne permanent anliegende Spannung überdauern, dennoch kann der Ausgangszustand, beispielsweise durch Spannungspulse umgekehrter Polarität, regeneriert werden. Derartige „Schalteffekte“ sind einer breiten Klasse von Materialien zu eigen und bergen großes Anwendungspotential. Sie werden häufig unter dem Begriff EPIR („Electric Pulse Induced Resistance“) geführt. ${ }^{\mathrm{b}}$

In diesem Kapitel erfolgt eine kurze phänomenologische und historische Einführung in elektrisch getriebene hysteretische Widerstandseffekte von Oxiden. Dabei wird auch auf die mögliche Verwendbarkeit als Speicherelement und das Memristor-Konzept eingegangen. Anschließend wird, insbesondere für Manganate und verwandte Oxide, ein Überblick der aktuell diskutierten Erklärungsansätze gegeben. Ergänzend zu dieser knappen Zusammen-

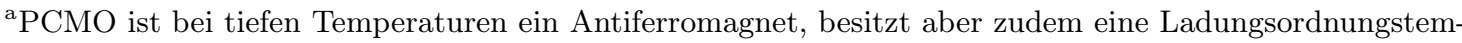
peratur $T_{\mathrm{CO}}$ noch oberhalb der Néel-Temperatur $T_{\mathrm{N}}$.

${ }^{\mathrm{b}}$ Leider hat sich in der Literatur bisher keine einheitliche Benennung elektroresistiver Effekte durchgesetzt. Einerseits wird zwischen CER und EPIR nicht immer klar abgegrenzt, andererseits wird EPIR von vielen Autoren mit einer bestimmten mikroskopischen Vorstellung des Schaltprozesses verbunden, obwohl das gleiche makroskopische Schaltverhalten je nach Material ganz verschiedene Ursachen haben könnte. 
fassung sind hier die Überblicksartikel von Sawa sowie von Waser \& Aono anzuführen. ${ }^{[6,7]}$ Es sei ferner auf eine Abhandlung von Waser et al. in Bezug auf das Anwendungspotential derartiger Effekte verwiesen. ${ }^{[8]}$

\subsection{Phänomenologische Einordnung}

Der in dieser Arbeit untersuchte Effekt gehört zur Klasse der hysteretischen Widerstandsänderungen als Folge eines anliegenden elektrischen Feldes oder der dadurch hervorgerufenen elektrischen Stromdichten. Hysteretisch bedeutet dabei zunächst nur, dass auf dem Hinund Rückweg einer Spannungsschleife verschiedene Stromverläufe gemessen werden. Als remanent bezeichnet man das Widerstandsschalten, wenn auch nach Zurückfahren des Feldes noch ein veränderter Widerstand vorliegt. Synonym wird der aus der Speichertechnologie entlehnte Begriff „,nonvolatil“ gebraucht. Naturgemäß bedeutet Remanenz hierbei nur, dass auf der Zeitskala der Messungen kein Abklingen der hervorgerufenen Änderung erkennbar ist.

Die Vielfältigkeit hysteretischer Widerstandsphänomene ist enorm: Es werden Widerstandsänderungen stark unterschiedlicher Ausprägung, mit oder ohne Abhängigkeit von der Polarität des treibenden Feldes oder auch der Dauer, welche es anliegt, beobachtet. Die Leitfähigkeit kann sich sowohl remanent als auch nur volatil ändern. Über verschiedenste Strom-Spannungs-Kennlinien - mit eher kontinuierlichem oder sprunghaftem, zum Teil sogar mehrstufigem Verhalten - ist berichtet worden.

Die untersuchten Strukturen sind dabei meist kondensatorartig aufgebaut: Zwischen zwei gleich- oder verschiedenartigen Metallelektroden befindet sich eine isolierende oder hochohmige Schicht. Eine derartige Metall/Isolator/Metall-Probe (MIM) ist in Abb. 2.2(a) skizziert. Bei der Untersuchung von Schaltphänomenen muss immer das gesamte derartige MIM-System berücksichtigt werden: Der Schalteffekt kann zwar eine inhärente Eigenschaft des isolierenden Filmes sein, so dass die Metallschichten wirklich nur als Zuleitungen dienen; meistens sind aber gerade die Grenzflächenbeschaffenheit und die Materialwahl der Elektroden von großer Bedeutung.

(a) Unipolar

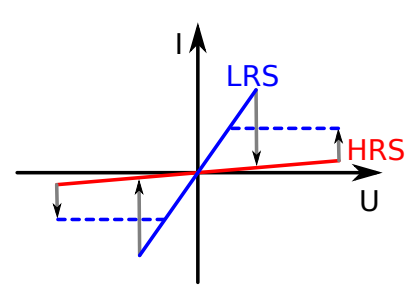

(b) Bipolar

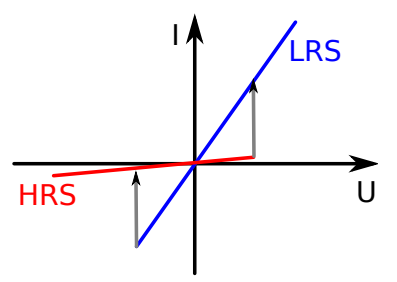

Abbildung 2.1: Unipolares und bipolares Schalten (schematisch). Nach [6].

Eine erste qualitative Einordnung des Schaltverhaltens erfolgt zunächst anhand der Form der $I$ - $V$-Hysterese. Man unterscheidet zwischen den beiden in Abb. 2.1 schematisch gezeigten Schalttypen. Falls der erreichte Widerstandszustand ausschließlich von der Amplitude des schaltenden Spannungspulses bestimmt ist - die $I$ - $V$-Kennlinien also symmetrisch 
erscheinen, so bezeichnet man das Schalten als unipolar. Ist hingegen auch die Polarität ausschlaggebend, so spricht man von bipolarem Schalten.

Die Polarität ist im bipolaren Falle oftmals durch eine offensichtliche Asymmetrie des Systems begründbar - beispielsweise vorgegeben durch verschiedene Kontaktmaterialien oder -flächen der Elektroden. Die Reihenschaltung zweier identischer bipolarer Schaltelemente in entgegengesetzter Orientierung - zu einem insgesamt also wieder symmetrisch konstruierten Element - kann jedoch Untersuchungen von Chen et al. zufolge dennoch auf begrenzten Spannungsintervallen ein bipolar erscheinendes Schaltverhalten zeigen. ${ }^{[79] c}$

\subsection{Anwendungsmöglichkeiten}

Die große Aufmerksamkeit, welche die oben beschriebenen Schaltphänomene seit einigen Jahren erfahren, ist nicht allein akademischer Natur, sondern liegt zu einem großen Teil in der Hoffnung auf technologische Nutzbarkeit in der Datenspeicherung begründet.

Das Funktionsprinzip des RRAM („Resistive Random Access Memory"), einem Kandidaten für einen zukünftigen nonvolatilen und reversiblen Massenspeicher, fußt auf der Verwendung memristiver Materialien. Ein RRAM-Speicher besteht, wie in Abb. 2.2 (b) skizziert, aus einer zweidimensionalen Anordnung von MIM-Elementen. Diese können über „Word lines“ und „Bit lines“ einzeln angesprochen und mittels großer Spannungen umgeschaltet werden. Kleine Spannungen erlauben das Auslesen des Widerstandszustandes.

Für eine industrielle Verwendung sind vor allem eine langfristige Remanenz, große Widerstandsunterschiede, kleine Schaltspannungen, schnelle Schaltprozesse und eine Lebensdauer von möglichst vielen Zyklen von Bedeutung. RRAM wird deshalb als mögliche Nachfolgetechnologie für den nonvolatilen Flash-Speicher gehandelt, dessen weitere Miniaturisierung bald an physikalische Grenzen zu stoßen droht; man erhofft sich außerdem Verbesserungen vor allem der Lebensdauer, der Schaltspannung und -geschwindigkeit. ${ }^{[8]}$

Das Potential resistiv schaltender Materialien ist jedoch nicht auf die binäre Speichertechnologie beschränkt. Probensysteme, welche sogenanntes „Multi-Level Switching“, also mehrstufige oder sogar kontinuierliche Schalteffekte zeigen, versprechen Anwendungen jenseits der Zweizustandslogik - es könnten auch analoge Speicherelemente realisiert werden.

Bisherige RRAM-Bauelemente haben nur prototypischen Charakter; in Ermangelung eines hinreichenden physikalischen Verständnisses der zugrundeliegenden Mechanismen ist jedoch bisher keine konkrete Entwicklungsvorgabe möglich um die Schalteigenschaften gezielt für die industrielle Nutzung zu verbessern.

\subsection{Historischer Abriss}

Bereits seit den 1920er Jahren sind thermisch phasenwechselnde Materialien mit einhergehenden Widerstandsänderungen bekannt. ${ }^{[80]}$ Die Entdeckung eines scharf und reversibel schaltbaren amorphen Chalkogenides, $\mathrm{Te}_{48} \mathrm{As}_{30} \mathrm{Si}_{12} \mathrm{Ge}_{10}$, im Jahre 1968 durch Ovshinsky ${ }^{[81]}$ gab Anlass zu einer breit angelegten Erforschung dieser Materialklasse und machte früh das

${ }^{c}$ Es kommt bei einer solchen „Minor Loop“ nur der Schalteffekt des einen Halbelementes zum Tragen, während der geringere Spannungsabfall der anderen, sich im metallischen Zustand befindlichen, Komponente nicht zum Schalten ausreicht. 
(a)

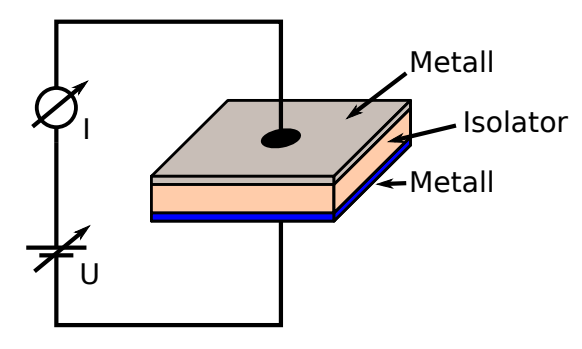

(b)

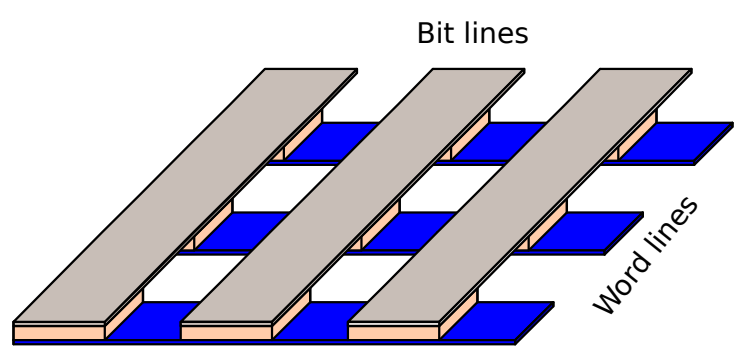

Abbildung 2.2: MIM-Schichtstruktur und Anwendung als RRAM. Die gegebenenfalls veschiedenen Metallschichten und die internen Grenzflächen der MIM-Sandwichstruktur (a) müssen als Bestandteil des Funktionselementes betrachtet werden und nicht allein als Kontaktierung. (b) Ein zweidimensionales Feld von über "Word lines" und „Bit lines" individuell adressierbaren MIM-Zellen bildet das Konzept des RRAMs. (Nach [7].)

Anwendungspotential als Speichermedium und damit die wirtschaftliche Relevanz derartiger Systeme deutlich.

Parallel wurden jedoch auch große Anstrengungen zum Verständnis und der Nutzung hysteretischer Widerstandsphänomene speziell an oxidischen Substanzen unternommen. In der ersten Dokumentation elektrisch induzierter Widerstandsänderungen an einem oxidischen System berichtete Hickmott im Jahre 1962 über seine Untersuchungen an $\mathrm{Al} / \mathrm{Al}_{2} \mathrm{O}_{3} / \mathrm{Au}$ Sandwichstrukturen. ${ }^{[82]}$ In den darauffolgenden Jahren wurde resistives Schalten an einer Reihe weiterer binärer Oxide wie etwa $\mathrm{SiO}^{[83]}$ und $\mathrm{NiO}^{[84]}$ beobachtet. Diese frühen Studien vergleichsweise einfacher Oxide wurden bis in die 1980er Jahre fortgesetzt und sind beispielsweise in den Übersichtsartikeln von Dearnaley et al., Biederman und Pagnia \& Sotnik zusammenfassend dargestellt. ${ }^{[85-87]}$

Zur Zeit erlebt dieses Forschungsgebiet einen erneuten Aufschwung, vor allem motiviert durch die Hoffnung auf Anwendungsmöglichkeiten in der Informationstechnologie. Dabei stehen jetzt auch zunehmend komplexere Oxide im Vordergrund der Untersuchungen. Ausgelöst wurde dieser Trend vor allem durch die Arbeiten von Asamitsu et al., welche 1997 für PCMO erstmals elektrisches Schalten an einem Manganat aufzeigten, sowie die Experimente von Beck et al. an Strontiumzirkonat. ${ }^{[3,88]}$

Die Reihe ternärer und komplexerer oxidischer Materialien, an welchen bisher elektrisch induziertes Widerstandsschalten beobachtet wurde, umfasst bei weitem nicht nur die in dieser Arbeit behandelten kolossal magnetoresistiven Manganate wie LSMO und LCMO und andere perowskitische Oxide wie beispielsweise Titanate, Cuprate und Zirkonate. Selbst an organischen Substanzen wurden ähnliche Effekte aufgefunden. ${ }^{89-91]}$

Angesichts der großen Vielzahl an zum Teil sehr verschiedenen Materialsystemen ist es nicht verwunderlich, dass für die resistiven Schalteffekte auch verschiedene Mechanismen verantwortlich sind. Es müssen individuelle Erklärungsansätze den jeweiligen materialspezifischen Eigenschaften Rechnung tragen. Die insbesondere in den vergangenen zehn Jahren intensivierten Bemühungen um ein mikroskopisches Verständnis der Schaltprozesse in Oxiden sind in Abschnitt 2.5 zusammengefasst. 


\section{4. Übergangsmetalloxide als memristive Systeme}

Von großer Bedeutung bei der Beschreibung hysteretischer Widerstandseffekte ist das Konzept des sogenannten „Memristors“. Bereits 1971 postulierte Chua aufgrund von Symmetrieerwägungen, dass neben Widerstand, Kapazität und Induktivität noch ein viertes fundamentales passives elektrisches Bauelement existieren müsse, welches den magnetischen Fluss $\phi$ und die Ladung $q$ gemäß $d \phi=M d q$ verknüpft. Ferner zeigte Chua mögliche schalttechnische Anwendungsfelder auf. ${ }^{[4]}$ Differentiation nach der Zeit ergibt folgende Gleichung:

$$
U=\frac{d \phi}{d t}=M(w) I \quad \text { mit }: \quad \frac{d w}{d t}=I .
$$

Die hier eingeführte Zustandsvariable $w$, welche die Memristanz $M$ definiert, ist offensichtlich identisch mit der elektrischen Ladung $q$. Ein Bauteil unveränderlicher Memristanz $M(q)=$ const ist von einem Ohmschen Widerstand phänomenologisch ununterscheidbar. Chua bezeichnete dieses hypothetische Bauelement mit dem Kofferwort „Memristor" aus „memory resistor", da es einen Widerstand mit Gedächtnisfunktion bezüglich seiner elektrischen Vorgeschichte darstellt. Trotz aufwendiger Suche gelang es erst 2008 Strukov et al. einen derartigen idealen Memristor herzustellen. ${ }^{[92]}$ Chua \& Kang führten 1976 ergänzend noch die allgemeinere Klasse der „,memristiven Systeme“ ein. ${ }^{[5]}$ Diese gehorchen folgenden Gesetzen:

$$
U=M(w, I) I \quad \text { mit : } \quad \frac{d w}{d t}=f(w, I) .
$$

Derartige elektrische Elemente, wobei $w$ jetzt einen Satz von gegebenenfalls mehreren Zustandsvariablen bezeichnet, weisen ebenfalls eine Abhängigkeit des elektrischen Widerstands von der Vorgeschichte auf, nicht jedoch unbedingt nur allein von der Ladung. Die hier untersuchten Manganate genügen dieser erweiterten Definition und sind deshalb in die Klasse der memristiven Systeme einzuordnen.

\subsection{Erklärungsansätze}

Über den für das elektrisch induzierte Schalten verantwortlichen Mechanismus gibt es in den meisten Fällen noch kein gesichertes und detailliertes Bild. Die im Folgenden geschilderten Mechanismen haben daher vor allem die Funktion von Arbeitshypothesen; es handelt sich außerdem nur um eine Auswahl möglicher Beschreibungen mit Schwerpunkt auf Übergangsmetalloxiden. Grundsätzlich unterscheidet man zwischen Schalteffekten, welche einem filamentären Wachstum beziehungsweise Unterbrechen eines leitenden Pfades geschuldet sind und solchen, wo sich der Widerstand einer Kontaktfläche ändert.

\subsubsection{Filamentäre Schalteffekte}

Filamentäre Schalteffekte, bei denen sich durch elektrochemische Prozesse ein metallischer Pfad aus dem Material einer aktiven Elektrode durch die isolierende Barriere zur 
Gegenelektrode bildet, sind schon seit längerem bekannt. Neben dieser direkten elektrochemischen Ausbildung von Metalldendriten durch eine Migration von Kationen werden in Oxiden auch anionische Mechanismen, allen voran die unten ausführlicher vorgestellte Sauerstoffmigration, erwogen. ${ }^{[6]}$

Begründet wird die Annahme eines filamentären Prozesses oftmals durch die Beobachtung eines „Trainingseffektes“ vor dem eigentlichen Schalten: Die Probe muss erst längere Zeit einer elektrischen Spannung ausgesetzt werden, bevor das Schaltverhalten einsetzt. Ein Hinund Herschalten ist danach jedoch auch mit kürzeren Pulsen möglich. Dieses Verhalten wird so interpretiert, dass zwischen den Elektroden zuerst ein leitfähiges Filament mittels einer Art „weichem" Spannungsdurchschlag gebildet wird, welches während der späteren Schaltprozesse nur noch an Engstellen - insbesondere an den Kontaktflächen - unterbrochen oder wieder verbunden werden muss. Die eindeutige Abbildung des Wachstums metallischer Filamente in isolierenden Oxiden ist über lange Zeit nicht gelungen. An NiO fanden Park et al. 2007 mittels hochaufgelöster TEM-Messungen starke Hinweise auf einen durch Filamentbildung entlang von Korngrenzen verursachten Schaltprozess. ${ }^{[93]}$

Für das Filamentwachstum werden in Oxiden zum Beispiel thermisch geförderte Redoxprozesse verantwortlich gemacht. Im Falle von undotiertem Strontiumtitanat, $\mathrm{SrTiO}_{3}$ (STO), wo es deutliche Indizien auf einen Schaltprozess filamentären Wesens gibt, wird vor allem Elektromigration von Sauerstoffleerstellen als mögliche Ursache des Effektes erwogen. ${ }^{94]}$

Filamentäre Schaltphänomene werden für Manganate eher selten in Betracht gezogen. Fors et al. studierten das Widerstandsschalten epitaktischer Heteroschichtstrukturen von der Form $\mathrm{Ag} / \mathrm{CeO}_{2} / \mathrm{La}_{0,67} \mathrm{Ca}_{0,33} \mathrm{MnO}_{3} .{ }^{[95]}$ Die dabei auftretenden starken Schalteffekte erklärten sie durch eine Änderung der Cer-Valenzen. Der Widerstandssprung erklärt sich dann als Mott-Übergang entlang eines Strompfades durch die sonst isolierende Barriere. Als Auslöser für die Valenzänderung betrachteten sie wiederum die feldgetriebene Migration von Sauerstoffleerstellen. Nach dieser Vorstellung, dient LCMO hier also nur als Reservoir für Sauerstoffionen - das Szenario beschreibt keinen Mechanismus für LCMO.

\subsubsection{Schalten an Grenz- oder Kontaktflächen}

Für viele, vor allem bipolar schaltende Materialsysteme - insbesondere auch die hier behandelten Manganate - wird kein filamentäres Durchschalten zwischen den Elektroden angenommen. Stattdessen geht man davon aus, dass nur der dominante Widerstandsbeitrag an der Grenzfläche zu den Zuleitungen reduziert wird. Impedanzexperimente von Baikalov et al. und Tsui et al. an einer Reihe von verschiedenen resistiv schaltenden Materialien, darunter auch PCMO und LCMO, konnten diese Vermutung stützen. Für PCMO wurde dabei eine Beschränkung auf einen Raumbereich von nicht mehr als $10 \mathrm{~nm}$ Dicke festgestellt. ${ }^{[96,97]}$ Für diesen flächenartigen Schalttyp kursieren verschiedene Erklärungsansätze.

\section{Elektromigration von Sauerstoffvakanzen}

Viele Deutungen enthalten die Annahme einer durch Elektromigration veränderlichen Sauerstoffleerstellendichte als zentrales Element. Dieser Aspekt wurde erstmals von Baikalov et al. in den Raum gestellt, um die in ihrem Falle beobachtete, vergleichsweise langsame Dynamik 
des Schaltvorgangs zu erklären. ${ }^{[96]}$ Da eine sehr starke Abhängigkeit des elektrischen Widerstandes von dem Sauerstoffdefizit in Manganaten bekannt ist ${ }^{\mathrm{d}}$ und die Oberfläche meist präpositionsbedingt sauerstoffdefizitär ist, ist es durchaus naheliegend, in der elektrisch getriebenen Migration von Sauerstoffvakanzen eine mögliche Ursache zu sehen.

Auf Tsui et al. geht die Beschreibung des Schalteffektes durch die Annahme einer variablen Dichte von Trapping-Zuständen in der Grenzschicht zur Elektrode zurück. ${ }^{[97]}$ Durch Untersuchungen der Spannungsabhängigkeit des Stromes in den verschiedenen Widerstandszuständen einer Reihe von schaltenden Materialien fanden sie - auch für das Manganat PCMO - eine ungefähr quadratische Abhängigkeit, was als Hinweis auf einen durch die Ausbildung einer Raumladungszone limitierten Transport („Space Charge Limited Current", SCLC) verstanden werden kann. Unter der Hypothese, dass sich der Potentialverlauf dieser „Fallen“ für die Ladungsträger beim Schalten nicht wesentlich ändert, kamen sie zu dem Schluss, dass sich die Dichte dieser Zustände durch den Schaltprozess ändern müsse. Hierfür nahmen auch sie wieder eine Elektromigration von Sauerstoffionen beziehungsweise -leerstellen als ursächlich an.

In ersten theoretischen Arbeiten untersuchten Rozenberg et al. elektrische Schalteffekte in einem phänomenologischen Modell eines räumlich inhomogenen Domänensystems. ${ }^{[99]}$ Ergänzungen durch Quintero et al. ergaben, basierend wieder auf der Annahme einer Migration von Sauerstoffvakanzen, dass der entscheidende Faktor für die Leitfähigkeitsvariation in einer daraus folgenden Änderung der lokalen Dotierung zu suchen ist. ${ }^{[100]}$

Über die Details, wie sich die hypothetische Änderung der Sauerstoffleerstellenkonzentration in einem Widerstandsschalten niederschlägt, existieren also sehr verschiedene Ansichten. Die Leerstellenmigration an sich wird jedoch gegenwärtig in der Fachliteratur als Auslöser der Schalteffekte in vielen Oxiden favorisiert.

\section{Schottky-Barrieren}

Demgegenüber steht die von Sawa et al. vorgetragene Beschreibung durch eine SchottkyBarriere an der Grenzfläche. ${ }^{[101]}$ Schottky-Barrieren sind dem $p$ - $n$-Übergang ähnliche MetallHalbleiter-Grenzflächen mit gleichrichtenden $I$ - $V$-Kennlinien. ${ }^{[102]}$ Charakteristisch ist dabei die Abhängigkeit der Barrierenhöhe von der Austrittsarbeit des Metalls. Sawa et al. untersuchten die Schalteigenschaften der Ti/PCMO-Grenzfläche und fanden stark hysteretisches Verhalten. ${ }^{[101]}$ Dabei ließ sich durch das Anlegen großer Spannung oder das wiederholte Durchlaufen von Spannungszyklen ein gleichrichtender Zustand induzieren. Ähnliche Resultate erhielten Fujii et al. für niobdotiertes Strontiumtitanat. [103]

Es ergab sich so ein Modell, wonach eine durch Ladungsakkumulation in Grenzflächenzuständen elektrisch kontrollierbare Schottky-Barriere mit PCMO als $p$-Halbleiter beziehungsweise $\mathrm{Nb}: \mathrm{SrTiO}_{3}$ als $n$-Halbleiter entsteht. Die in diesem Falle eigentlich unintuitive besondere Eignung von Titan- im Vergleich zu beispielsweise Silber-Elektroden ${ }^{\mathrm{e}}$ für PCMO wurde mit der starken Affinität zwischen Sauerstoff und Titan begründet. Demnach könnte eine zusätzliche Sauerstoffverarmung unter der als ,Sauerstoffgetter“ fungierenden

\footnotetext{
${ }^{\mathrm{d}}$ Für $\mathrm{La}_{0,67} \mathrm{Ba}_{0,33} \mathrm{MnO}_{z}$ wurde beispielsweise eine Widerstandsvariation über vier Größenordnungen bei einem Sauerstoffdefizit von $5 \%$ festgestellt. ${ }^{[98]}$

${ }^{\mathrm{e}}$ Titan und Silber haben in etwa die gleiche Austrittsarbeit von $\approx 4,3 \mathrm{eV}$.
} 
Titanschicht eine erhöhte Dichte von Oberflächenzuständen erzeugen. Der Schalteffekt selbst beruht aber in diesem Modell nicht primär auf Elektromigration.

\section{Feld- oder stromgetriebene strukturelle Übergänge}

Die in vielen Übergangsmetalloxiden starke gegenseitige Beeinflussung struktureller und elektronischer Eigenschaften fand bei der Diskussion des Widerstandsschaltens bis heute erstaunlich wenig Berücksichtigung. Insbesondere für die hochkorrelierten, zur Phasenseparation neigenden Manganate, wo zudem spezielle Grenzflächeneffekte bekannt sind, liegt es eigentlich nahe, das Widerstandsschalten als eine weitere Ausprägung der Konkurrenz verschiedener struktureller und elektronischer Phasen zu verstehen.

Driscoll et al. beobachteten memristive Effekte an Vanadiumoxid $\left(\mathrm{VO}_{2}\right)$ und wählten einen ähnlichen Erklärungsansatz. ${ }^{[104]}$ Dieses Material zeigt ebenso wie die Manganate einen thermischen Metall-Isolator-Übergang. Er ist mit einem strukturellen Übergang des Materials verknüpft. ${ }^{[38]}$ Eine beobachtete Temperaturhysterese im Widerstandsverlauf führte zu der Hypothese, es handele sich bei dem Widerstandsschalten um ein Kennzeichen desselben thermisch induzierten Phasenüberganges, welcher sich aber nur durch Heizeffekte in nanoskaligen Bereichen entlang des perkolativen Strompfades ereignet (strenggenommen also ein eher filamentäres Bild).

In Verbindung mit Experimenten an PCMO als prototypischem RRAM-Element wurde beispielsweise von Hsu et al. auf eine mögliche Schlüsselrolle der Jahn-Teller-Verzerrungen hingewiesen. ${ }^{[105]}$ TEM-Untersuchungen von Jooss et al. an PCMO belegten erstmals eindeutig, dass mit dem Schalten auch Übergänge zwischen verschiedenen Überstrukturen durch eine kollektive Verdrehung und Verkippung der Sauerstoffoktaeder einhergehen. ${ }^{[106]}$ In Bezug auf LSMO ist in diesem Zusammenhang auch auf die Arbeiten von Esseling und Moshnyaga et al. für nanokolumnare Proben zu verweisen. ${ }^{[107,108]}$ 
Es erfolgt nun eine Beschreibung der verwendeten experimentellen Methoden, dabei wird nur sehr knapp auf die Probenpräparation und die Charakterisierung durch Standardmethoden der Festkörperphysik eingegangen. Der Schwerpunkt liegt auf der Vorstellung der nicht so verbreiteten C-AFM-Methode („Conductive Atomic Force Microscope“, leitfähiges atomares Kraftmikroskop), einer Variante der atomaren Kraftmikroskopie, welche für diese Arbeit intensiv genutzt wurde. Dabei wird nach dem Messkonzept die instrumentelle Realisierung des verwendeten kommerziellen Gerätes dargestellt und abschließend, auf Basis einer einfachen mechanischen Modellierung, die Größe des elektrischen Kontaktes für den verwendeten Spitzentyp abgeschätzt.

\subsection{Metallorganische Aerosoldeposition (MAD)}

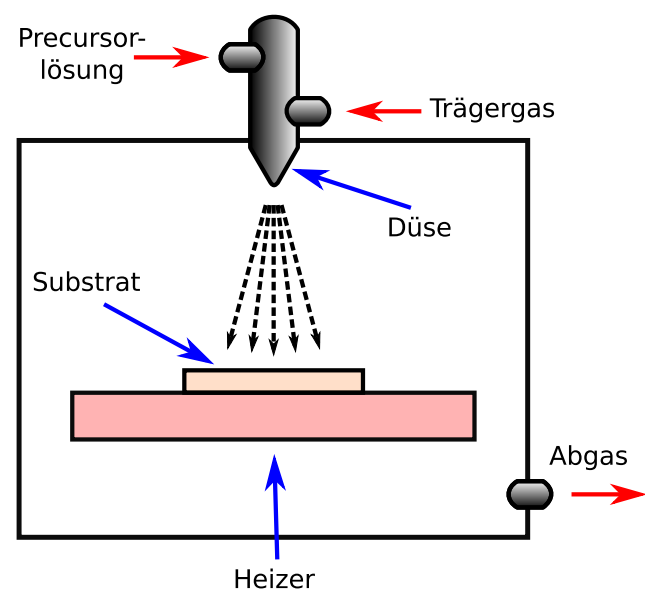

Abbildung 3.1: Herstellungsprinzip der metallorganischen Aerosoldeposition (schematisch, nach [109]).
Die metallorganische Aerosoldeposition ${ }^{[109]}$ (MAD) ist eine Herstellungsmethode für oxidische Dünnschichtproben; alle hier untersuchten Filme wurden mittels MAD produziert. Das Verfahren unterscheidet sich von den meisten anderen Methoden vor allem dadurch, dass es nicht unter Vakuumbedingungen betrieben wird. Ursprünglich konzipiert zur Präparation von Hochtemperatursupraleitern ${ }^{[110]}$ besticht es so auch bei der Herstellung anderer oxidischer Systeme wie den hier untersuchten Manganaten, weil Probleme wie das unter Vakuumbedingungen schwer vermeidbare stöchiometrische Sauerstoffdefizit umgangen werden können. Darüberhinaus können vergleichsweise großflächige Beschichtungen durchgeführt und schnelles Schichtwachstum (typischerweise etwa $10 \mathrm{~nm} / \mathrm{min}$ ) erzielt werden. 
Die Vorgehensweise ist in Abb. 3.1 skizziert. Zunächst werden kommerzielle MetallAcetylacetonate der Manganatbestandteile im entsprechenden Mengenverhältnis in Dimethylformamid gelöst. ${ }^{a}$ Diese Lösung wird in einer pneumatischen Düse unter Zuführung eines Trägergases, hier trockener Druckluft, zu einem feinen Aerosol zerstäubt; die Tröpfchengröße liegt, je nach Gasfluss, typischerweise bei einigen zehn Mikrometern. ${ }^{[109]}$ Trifft das Aerosol auf das bis zu $900{ }^{\circ} \mathrm{C}$ heiße Substrat, so werden die Precursorkomplexe pyrolytisch aufgebrochen und Metallionen scheiden sich auf dem Substrat ab. Die Luftatmosphäre führt dabei zur sofortigen Oxidation der Ionen auf der Oberfläche und der Verbrennung der organischen Precursorreste.

\subsection{Charakterisierung}

In diesem Abschnitt wird ein Überblick der verwendeten Standard-Methoden zur Charakterisierung der Manganatproben gegeben. Er ist auf die Röntgendiffraktometrie (und -reflektometrie) und Transportmessungen beschränkt. Auf eine Beschreibung der als bekannt vorauszusetzenden Rastertunnelmikroskopie wird verzichtet, stattdessen wird im anschließenden Abschnitt ja mit der C-AFM-Methode ein eng verwandtes Rastersondenverfahren vorgestellt.

\subsubsection{Röntgenstrukturanalyse}

Zur strukturellen Charakterisierung der Manganatproben wurden zwei kommerzielle, in der sogenannten Bragg-Brentanooder $\theta$-2 $\theta$-Geometrie arbeitende Röntgendiffraktometer benutzt: Das D5000[111] von Siemens und das D8 Advance ${ }^{[12]}$ von Bruker AXS.

Beide Anlagen verfügen über eine Kupferanode als Röntgenquelle, durch Monochromatisierung wird die $\mathrm{Cu}-\mathrm{K}_{\alpha}$-Linie der Wellenlänge $\lambda \approx 1,542 \AA$ herausgegriffen. Wie in Abb. 3.2 gezeigt, werden durch jeweils gleichzeitige Verkippung der Probe um den Winkel $\theta$ und Rotation des Detektors um $2 \theta$ nur Reflexe mit oberflächennormalen Streuvektoren $\vec{Q}$ registriert. Die beiden nachfolgend vorgestellten Betriebsmodi geben Aufschluss auf die Gitterstruktur und Schichtdicke der untersuchten Systeme, für detailliertere Einführungen sei auf Standardwerke der Festkörperphysik verwiesen. ${ }^{[113]}$

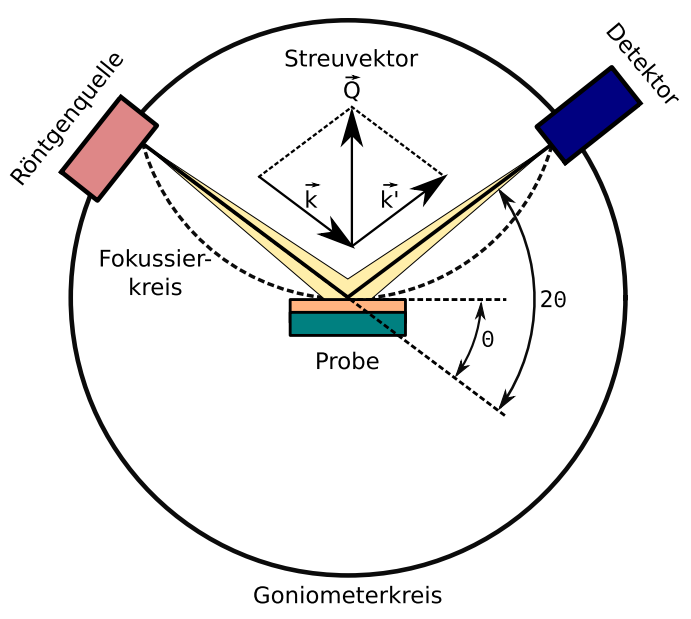

Abbildung 3.2: Funktionsweise eines Röntgendiffraktometrs in Bragg-BrentanoGeometrie (schematisch). Eingetragen ist auch die Orientierung der ein- und ausfallenden Wellenvektoren $\vec{k}$ und $\overrightarrow{k^{\prime}}$ und des resultierenden, oberflächennormalen Streuvektors $\vec{Q}=\overrightarrow{k^{\prime}}-\vec{k}$.

\footnotetext{
${ }^{a} \mathrm{Da}$ die Depositionsraten durch die vom Mischungsverhältnis abhängige Reaktionskinetik bestimmt werden, ist in der Regel eine von dem gewünschten stöchiometrischen Verhältnis abweichende, aus Erfahrungswerten begründete Komposition der Precursorlösung zu wählen.
} 


\section{Weitwinkelanalyse}

Für ausreichend große Winkel, $2 \theta>10^{\circ}$, ergeben sich unter Annahme elastischer Streuung, das heißt betragsgleicher einfallender und ausfallender Wellenvektoren $\vec{k}$ und $\overrightarrow{k^{\prime}}$, im Vergleich zu reziproken Netzebenenabständen große Streuvektoren $\vec{Q}=\frac{4 \pi}{\lambda} \sin \theta$. Die Streuintensität des Röntgenlichts hängt dann in folgender Weise von der für kristalline Materialien mit den Gittervektoren $\vec{R}$ translationssymmetrischen Elektronendichte $\rho(\vec{r})=\rho(\vec{r}+\vec{R})$ ab und erlaubt die Untersuchung atomarer Abstände:

$$
I(\vec{Q}) \propto\left|\int_{V} \rho(\vec{r}) e^{-i \vec{Q} \cdot \vec{r}} d \vec{r}\right|^{2}=|F(\vec{Q})|^{2} \cdot\left|\sum_{\vec{R}} e^{-i \vec{Q} \cdot \vec{R}}\right|^{2} .
$$

Intensitätsmaxima können somit nur dann auftreten wenn der Streuvektor $\vec{Q}$ gerade einem reziproken Gittervektor entspricht. Der Strukturfaktor $F(\vec{Q})$, die fouriertransformierte elektronische Aufenthaltsdichte in der Einheitszelle, beschreibt die mögliche Auslöschung von Reflexen, falls bereits innerhalb der Einheitszelle eine destruktive Überlagerung entlang der entsprechenden Richtung stattfindet. Für kubische Materialien der Gitterkonstanten $a$ und mit nach der Millerschen Indizierung bezeichneten Netzebenenabständen $d_{h k l}=$ $a / \sqrt{h^{2}+k^{2}+l^{2}}$ erhält man für die Streuwinkel $\theta_{h k l}$ der Intensitätsmaxima:

$$
\sin \left(\theta_{h k l}\right)=\frac{\lambda}{2 d_{h k l}} .
$$

Die Weitwinkelanalyse erlaubt somit die Bestimmung der Netzebenenabstände senkrecht zur Probenoberfläche und damit der entsprechenden Gitterkonstanten.

\section{Kleinwinkelanalyse}

In diesem Fall wird die Probe mit dem nur streifend einfallenden Röntgenstrahl untersucht. Durch den im Vergleich zu Weitwinkelmessungen reduzierten elastischen Streuvektor $\vec{Q}=$ $\frac{4 \pi}{\lambda} \sin \theta$ geht die Sensitivität auf dem Längenbereich atomarer Abstände verloren, der Film wirkt als homogenes Medium mit dem materialspezifischem Brechungsindex

$$
n=1-\delta-i \beta .
$$

Hierbei steht $\delta$ für die Dispersion des Brechungsindexes und $\beta$ für die Absorption. Maßgeblich für die Intensität sind in dieser Kontinuumsnäherung jetzt die Brechung und Reflexion an den Grenzflächen zwischen verschiedenen Materialien. Diese Methode der Röntgenreflektometrie ist daher empfindlich auf Abstände zwischen den jeweiligen Grenzflächen, hier zwischen Luft und Manganat sowie zwischen Manganat und Substrat, also die Schichtdicke. Nach Segmüller hängen die Einfallswinkel $\theta_{i}$ für extremale Intensitäten in folgender Weise mit der Schichtdicke $d$ zusammen: ${ }^{[114]}$

$$
\theta_{i}^{2}=2 \delta+\left(m_{i}+\Delta m\right)^{2} \frac{\lambda^{2}}{4 d^{2}}
$$

Hierbei kennzeichnet das ganzzahlige $m_{i}$ die Ordnung des Extremums, $\Delta m$ nimmt im Falle eines Phasensprungs um $\lambda / 2$ an der Grenzfläche zum Substrat für Maxima den 
Wert 1/2 und für Minima 0 an, andernfalls umgekehrt. Zur tatsächlichen Bestimmung der Schichtdicke wird ein auf Hink \& Petzold zurückgehendes Regressionsverfahren auf Basis der Methode der kleinsten Quadrate verwendet. ${ }^{[115]}$

\subsubsection{Transportmessungen}

Für makroskopische Messungen der Temperatur- und Magnetfeldabhängigkeit des elektrischen Widerstands wurden verschiedene kommerzielle Kryostaten verwendet, vor allem ein Physical Properties Measurement System von Quantum Design und ein Maglab von Oxford Instruments. Als Kryoflüssigkeit dienten dabei Helium und Stickstoff; Magnetfelder mit Flussdichten von mehreren Tesla werden in supraleitenden Magnetspulen erzeugt. Die Messungen erfolgten in der Regel in der sogenannten Vierpunktgeometrie um Beiträge der Leitungs- und Kontaktwiderstände zu minimieren. Die Kontaktierung geschah mittels Leitsilber.

\subsection{Atomare Kraftmikroskopie (AFM)}

\subsubsection{Grundlagen}

Während das 1981 von Gerd Binnig und Heinrich Rohrer erfundene Rastertunnelmikroskop (Scanning Tunneling Microscope, STM) längst als oberflächenwissenschaftliche Standardmethode Einzug in unzählige Anwendungsfelder gefunden hat und mit dem gemeinsamen Nobelpreis 1986 gewürdigt wurde, finden viele andere rastersondenmikroskopische Verfahren als sehr leistungsstarke und hochspezialisierte Instrumente auf einzelnen, zum Teil eng begrenzten Fachgebieten Anwendung. Es wird an dieser Stelle von einer Beschreibung des etablierten Rastertunnelmikroskops abgesehen und stattdessen exemplarisch auf die von Güntherodt \& Wiesendanger editierte Sammlung (siehe [116-118]) verwiesen, die einen umfangreichen Überblick des Themas vermittelt.

Die hier benutzte C-AFM-Methode ist ein Abkömmling des atomaren Kraftmikroskops (Atomic Force Microscope, AFM). Das AFM wurde 1986 von Gerd Binnig, Calvin Quate und Christoph Rohrer vorgestellt; erste kommerzielle Geräte waren schon ab 1989 verfügbar. Während die Anwendbarkeit des STM prinzipiell auf metallische und halbleitende Proben beschränkt ist, können durch Einsatz des AFM auch isolierende Oberflächen untersucht werden. Hierzu wird ein Biegebalken an die Probe angenähert - wir bedienen uns von nun an des hierfür auch im Deutschen gebräuchlichen englischen Terminus Cantilever.

Am Ende des Cantilevers befindet sich eine sehr scharfe Spitze, der sphärische Krümmungsradius beträgt je nach Fabrikat nur etwa einen bis einige zehn Nanometer. Tritt die Cantileverspitze in Wechselwirkung mit der Probenoberfläche, so bewirken die resultierenden attraktiven oder repulsiven Kräfte eine Verbiegung des Cantilevers, welche (äquivalent dem Tunnelstrom beim STM) als Rückkopplungsgröße in den Regelkreis eingeht. Neben der Auslenkung durch abstoßende Kontaktkräfte nahe der Probe können auch langreichweitige Wechselwirkungen detektiert werden. Letztere äußern sich in einer Reduktion der Resonanzfrequenz und -amplitude einer unter festem Abstand zur Probe oszillierenden Spitze. 
Hierfür ist eine geeignete Erfassung der feinen und schnellen Cantileverauslenkungen erforderlich. In der Anfangszeit wurde unter anderem versucht, diese durch eine über dem Cantilever angebrachte Tunnelspitze mittels der exponentiellen Abstandsabhängigkeit des Tunnelstromes aufzuzeichnen. Die gegenwärtig gebräuchlichsten Verfahren sind stattdessen optischer Natur: Man bedient sich entweder eines interferometrischen Aufbaus oder misst wie hier - die Ablenkung eines am Cantilever reflektierten Laserstrahles. Im „Kontaktmodus“ wird die Probe für topographische Messungen wie beim STM in $x$ - und $y$-Richtung mittels Piezostellelementen abgerastert, während ein z-Piezo den Abstand so einregelt, dass eine konstante Cantileververbiegung und somit Wechselwirkungskraft zwischen Spitze und Probe resultiert. Im Nichtkontaktmodus hält der Regelkreis die Resonanzfrequenz oder -amplitude konstant. Unter Annahme einer unter konstantem Abstand gleichbleibenden Wechselwirkung zwischen Spitze und Probe können die Nachregelungen des z-Piezos auch hier als Höhenkarte interpretiert werden.

Neben der bloßen Probentopographie kann durch Verwendung geeigneter, funktionalisierter Spitzen auch eine Vielzahl anderer physikalischer Größen lateral hochaufösend gemessen werden; wie beim Rastertunnelmikroskop sind auch hier neben dem bildgebenden Ansatz auch spektroskopische Messungen und Kombinationen von beidem von großer Bedeutung. Für eine Übersicht der Rasterkraftmikroskopie sei hier die Monographie von Meyer et $a l$. angefürt. ${ }^{[119]}$ Neben den breit gefächerten analytischen Kapazitäten der verschiedenen Betriebsmodi bietet die Rastersondenmikroskopie die Möglichkeit der gezielten Modifikation der Probenoberfläche auf kleinster Skala - von mechanischen Indentationen über das Anlegen elektrischer Spannungen oder den mit dem Stromfluss einhergehenden Wärmeeintrag bis hin zur gezielten Manipulation von Adatomen mittels STM. Nachfolgend werden die mechanischen Vorgänge bei Annäherung des Cantilevers an die Probe beschrieben. Es folgt eine Vorstellung der auf dem Kontaktmodus basierenden C-AFM-Methode und des verwendeten kommerziellen Mikroskops. Den Abschluss bildet eine Untersuchung der mechanischen und elektrischen Kontaktbedingungen.

\subsubsection{Annäherung der AFM-Spitze zum Kontaktmodus}

Wir wollen jetzt ein qualitatives Verständnis der Vorgänge während der Annäherung der Cantileverspitze zur Probenoberfläche erarbeiten. Die Abstandsabhängigkeit der zwischen der AFM-Spitze und der Probe auftretenden Kräfte ist ein sehr umfangreiches Gebiet und bietet wertvolle experimentelle Zugänge zu den Oberflächeneigenschaften der untersuchten Systeme. Im Folgenden soll nur ein kurzer phänomenologischer Eindruck vermittelt werden, eine umfassende Einführung auch in die theoretischen Konzepte der Kontaktmechanik ist in den Abhandlungen von Cappella \& Dietler ${ }^{[120]}$ sowie von Sarid ${ }^{[121]}$ zu finden.

AFM-Cantilever sind üblicherweise aus Si oder $\mathrm{Si}_{3} \mathrm{~N}_{4}$ gefertigt. Ihre Länge $L$ beträgt in der Regel wenige hundert, die Breite $w$ einige zehn und die Dicke $t$ nur wenige Mikrometer. ${ }^{\mathrm{b}}$ Für einen Elastizitätsmodul $E$ ergibt sich nach der Elastizitätslehre eine Federkonstante::121]

$$
k=\frac{E t^{3} w}{4 L^{3}} .
$$

\footnotetext{
${ }^{\mathrm{b}}$ Wir beschränken uns hier auf den verwendeten rechteckigen Cantilevertyp - es gibt daneben auch V-förmige Fabrikate.
} 
Die Kenntnis dieser Federkonstanten erlaubt es, aus der detektierbaren Verbiegung des Cantilevers quantitative Rückschlüsse auf die wirkenden Kontaktkräfte zu ziehen; im dynamischen Modus bestimmt sie die Eigenfrequenz des schwingenden Cantilevers. Wir betrachten hier exemplarisch die aus einem Lennard-Jones-Potential resultierende Kraft von der in Abb. 3.3 (a) gezeigten Form $F(z)=-\frac{A}{D^{7}}+\frac{B}{D^{13}}$, um die Vorgänge bei Annäherung der Spitze qualitativ zu verstehen. Das empirische, interatomare LennardJones-Potential setzt sich aus der attraktiven Van-der-Waals-Kraft und der konkurrierenden Pauli-Repulsion zusammen. Zur Beschreibung der tatsächlichen Wechselwirkung zwischen der Cantileverspitze und der Probe ist es ungeeignet: Beides sind makroskopisch ausgedehnte Objekte; die Aufintegration der atomaren Van-der-Waals-Kräfte hierüber ergibt so zum Beispiel deutlich schwächere Abhängigkeiten, im Falle einer Kugel über einer Ebene etwa nur einen $D^{-2}$-Beitrag zur Kraft. ${ }^{[121]}$

Abb. 3.3 (b) zeigt beispielhaft eine Messung der Kraft, welche die Cantileverspitze erfährt, in Abhängigkeit von der Verschiebung des Piezos. Der für derartige Auftragungen gebräuchliche Ausdruck „Kraft-Abstands-Kurve“ ist dabei insofern missverständlich, als nicht über den Abstand $D$ zwischen der Spitze und der Probe aufgetragen wird, sondern über den Verfahrweg $z$ des Piezos - korrekter ist daher der Begriff „Kraft-VerschiebungsKurve". Die Spannungsabhängigkeit der Piezostrecke $z$ ist nämlich für die instrumentellen Abmessungen und das jeweilig verwendete piezoelektrische Material bekannt, während der Abstand $D$ eine Kombination aus der Piezostrecke $z$ und der vom Oberflächenpotential der Probe bewirkten Cantileververbiegung ist.

(a) Kraft in Folge eines Lennard-Jones-Potentials

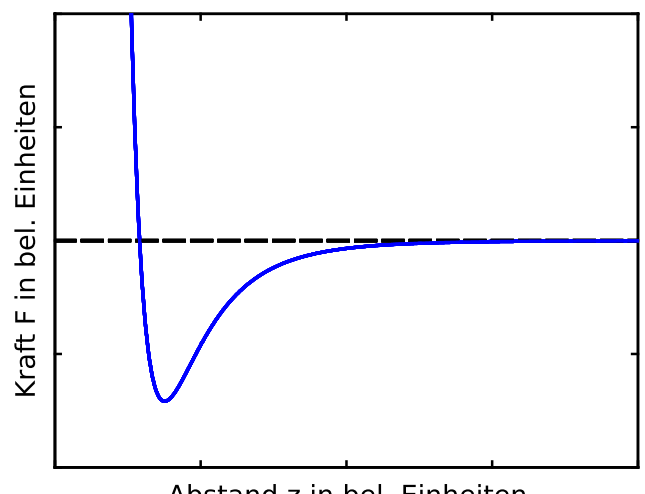

Abstand $z$ in bel. Einheiten (b) Kraft-Verschiebungs-Kurve (gemessen)

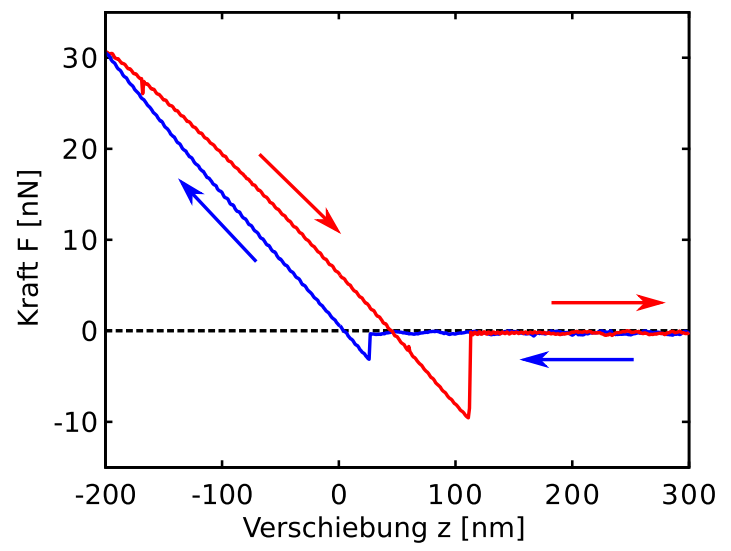

Abbildung 3.3: Die hier exemplarisch gezeigte Kraft durch ein Lennard-Jones-Potential (a) ist für sehr kleine Abstände stark repulsiv, danach attraktiv von rasch abfallender Stärke. Bei der Kraft-Verschiebungs-Kurve (b) zeigt die blaue Linie den Annäherungsverlauf, die rote den beim Zurückziehen des Cantilevers. (Die vorliegende Hysterese zwischen Hin- und Rückweg im Kontaktregime ist nicht in plastischen Verformungen begründet, sondern in einem Nachkriechen des Piezos - ein für das Gerät typisches Artefakt.[122]) 
Die exemplarisch gezeigte Kraft-Verschiebungs-Kurve nimmt den folgenden Verlauf:

1. Für große Abstände wirkt noch keine Kraft zwischen Spitze und Probe; der Cantilever verweilt in seiner Ruheposition.

2. Bei ausreichender Annäherung bewirken die langreichweitigen Van-der-Waals-Kräfte ein abruptes Heranspringen an die Oberfläche, der Cantilever wird zur Probe hin verbogen und eine negative Kraft wird detektiert. Die Verbiegung folgt näherungsweise dem Hookeschen Gesetz, bei weiterer Annäherung steigt die Kraft also linear an.

3. An dem Punkt, wo sich die repulsiven und attraktiven Anteile des Oberflächenpotentials gerade kompensieren, wirkt keine Nettokraft mehr und der Cantilever befindet sich in seiner Ruhelage.

4. Für positive Kräfte - im Regime abstoßender Wechselwirkung - geht neben der Verformung des Cantilevers auch eine elastische Gegenkraft bei Indentation der Probe ein.

5. Beim Zurückziehen des Piezos erfolgt eine lineare Abnahme der detektierten Kraft, wieder bis in den negativen Bereich.

6. Beim Überkommen der Adhäsionskräfte verliert die Cantileverspitze schließlich den mechanischen Kontakt und kehrt in ihre Ruheposition zurück.

Plastische Verformungen der Probe äußern sich gegebenenfalls in einer zusätzlichen Hysterese zwischen dem Hin- und Rückweg. Die sich auf dem Hinweg im Kontaktregime ereignenden Deformationen bewirken eine Verringerung der detektierten Verbiegung im Vergleich zum Hookeschen Verhalten. Nach dem Umkehrpunkt erfolgt dann eine raschere Abnahme der Kraft als auf dem Hinweg.

Die Aufnahme topographischer Messungen erfolgt unter konstanter Andruckkraft - im Rahmen dieser Arbeit wurden typischer wenige Nanonewton angelegt. Zu geringe Kräfte erhöhen das Risiko, den Kontakt während der Messung zu verlieren, während man bei zu großen Kräften Gefahr läuft, die Probenoberfläche plastisch zu verändern - der resultierende Druck kann aufgrund der kleinen Kontaktfläche sehr groß sein. Falls, wie beim C-AFM, funktional beschichtete Cantilever verwendet werden, bewirken zu hohe Andruckkräfte außerdem eine raschere Alterung der Beschichtung.

\subsubsection{Die C-AFM-Methode}

Die Verwendung leitfähig beschichteter Cantilever eröffnete schon kurz nach Entwicklung des Rasterkraftmikroskops eine Reihe von wertvollen konzeptionellen Erweiterungen der AFM-Technik. Elektrische Oberflächencharakteristika wie beispielsweise die Kapazität (mittels Scanning Capacitance Microscopy ${ }^{[123]}$, SCM) oder das elektrostatische Potential (Kelvin Probe Force Microscopy ${ }^{[124,125]}$, KFM) können mit hoher örtlicher Auflösung studiert werden. Neben diesen Variationen des dynamischen Modus können metallische Spitzen auch direkt im mechanischen Kontakt genutzt werden. Hier ist beispielsweise die Untersuchung 
der Verformung piezoelektrischer Proben unter anliegender Wechselspannung zu nennen (Piezo Response Force Microscopy, PFM).

Der auf den ersten Blick einfachste Fall ist jedoch die direkte Durchführung von Transportmessungen über den nur nanometergroßen Kontaktbereich. Diese Methode, welche etwa seit Mitte der letzten Dekade des vorigen Jahrhunderts Anwendung findet, bezeichnen wir hier als C-AFM. ${ }^{c}$ Das in Abb. 3.4 skizzierte Funktionsprinzip ist bestechend simpel: Ein leitfähig beschichteter Cantilever wird genutzt, um auf herkömmliche Weise im AFM-Kontaktmodus eine Höhenkarte aufzuzeichnen. Gleichzeitig wird unter permanent anliegender Gleichspannung der Strom gemessen. Im Gegensatz zur Rastertunnelmikroskopie sind Topographie und Strom hier also unabhängige Messgrößen.

In der vorliegenden Realisierung - siehe unten - besteht die Gegenelektrode aus großflächigen Kontakten am Probenrand. Der dominante Einfluss auf den gemessenen Strom entstammt demnach den Kontaktverhältnissen und inbesondere der lokalen Leitfähigkeit an der Spitzenposition. Die simultan mit der Topographie aufgenommenen Stromkarten können daher in sehr guter Näherung als qualitative Karten der Oberflächenleitfähigkeit angesehen werden.

Durch den AFM-Ansatz ist es sogar möglich, Proben sehr heterogener elektrischer Leitfähigkeit kontrastreich zu charakterisieren, selbst wenn auf den isolierenden Regionen keine Tunnelmikroskopie möglich ist. Anfängliche Arbeiten konzentrierten sich vor allem auf die Charakterisierung von Halbleiteroberflächen, insbesondere Silicium. So nutzten beispielsweise Shafai et al. diese Methode, um ortsabhängig die Art und Stärke der Dotierung zu analysieren ${ }^{[126]}$ und Murrell et al. studierten das elektrische Durchschlagsverhalten von $\mathrm{SiO}_{2}$-Gatekontakten. ${ }^{[127]}$

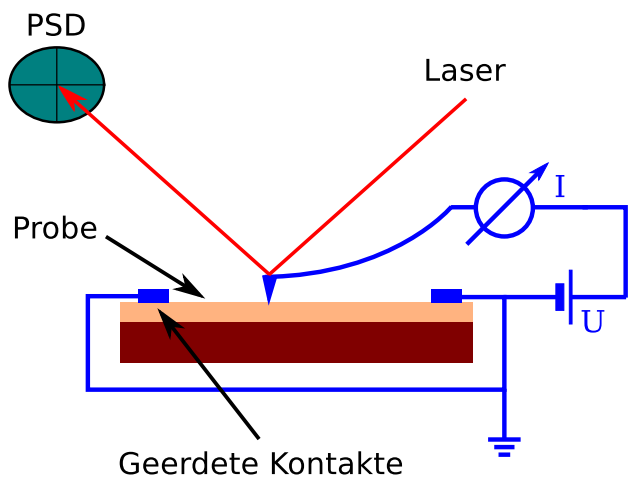

Abbildung 3.4: Funktionsweise der C-AFM-Methode. Die im AFM-Kontaktmodus die Oberfläche abrasternde Cantileverspitze wird gleichzeitig zu elektrischen Messungen genutzt; die asymmetrischen Kontaktverhältnisse ergeben ein hohes örtliches Auflösungsvermögen.

\footnotetext{
${ }^{\mathrm{c}}$ In der Fachliteratur wird diese AFM-Variante leider nicht einheitlich nur als C-AFM bezeichnet; es kursieren noch andere Namen und Abkürzungen, wie zum Beispiel Scanning Resistance Microscopy (SRM), für Verfahren, die sich vom hier beschriebenen Prinzip nicht oder nur geringfügig unterscheiden.
} 
Trotz der aufgeführten Vorzüge und ersten Erfolge erfreut sich C-AFM im Vergleich zur Rastertunnelspektroskopie bis heute einer ungleich kleineren Beliebtheit. Dies liegt zum einen sicherlich an den durch die Beschichtung wesentlich vergrößerten Spitzenradien und der daraus folgenden signifikanten Verschlechterung des topographischen Auflösungsvermögens. Darüberhinaus ist aber auch die Beschaffenheit des elektrischen Kontaktes selbst häufig unklar: ${ }^{[128]}$ Intuitiv vermutet man zwar eine direkte Kontaktierung mit Ohmscher Strom-Spannungs-Charakteristik. Tatsächlich können jedoch durch Kontamination und Korrosion der Spitzen- und Probenoberfläche sowie mechanischen Abtrag der metallischen Beschichtung gänzlich andere Kontaktbedingungen entstehen. Neben einer Tunnelbarriere durch Oxidation der Spitzen- oder Probenoberfläche sind auch sehr dünne, selbst unter Vakuumbedingungen nicht unbedingt vermeidbaren Kontaminationsfilme keine Seltenheit. Eine derartige Benetzung kann sowohl einen zusätzlichen Widerstand darstellen, als auch parasitäre Ströme ermöglichen.

Es gibt viele Bemühungen, die Kontaktverhältnisse zu charakterisieren oder experimentell zu definieren, das Durchbrechen einer Kontaminationsschicht kann beispielsweise durch einen plötzlichen Anstieg des Stromes bei Erhöhung der Andruckkraft festgestellt werden. Andere Ansätze vergleichen die Beschreibbarkeit der Kontaktverhältnisse als Sharvinscher Punktkontakt $^{\mathrm{d}}$ mit ballistischen Transporteigenschaften mit der als Tunnelkontakt gemäß der Simmons-Formel. ${ }^{[130]}$ Für sehr starke Kräfte, wenn auch der Kontaktradius deutlich vergrößert und eine Kontaminationslage auf jeden Fall durchdrungen ist, liegt ein Ohmscher Kontakt mit diffusivem Transportverhalten vor. ${ }^{[119]}$

Ein sehr wichtiges Kriterium ist naheliegenderweise der Verlauf der Strom-SpannungsKennlinie, der sich im Ohmschen Fall linear verhalten sollte. Für Manganate ist eine derartige Charakterisierung leider erschwert, weil die Kennlinien schon intrinsisch einen stark nichtlinearen Charakter haben, was durch eine strominduzierte Aufheizung noch verstärkt werden kann. Im Rahmen dieser Arbeit werden alle C-AFM-Ergebnisse daher ausschließlich qualitativ interpretiert. Durch die Verwendung der nachfolgend dargestellten Vakuumanlage kann allerdings davon ausgegangen werden, dass die Probenoberfläche nicht durch Flüssigkeitsfilme, sondern nur durch sehr dünne Kontaminationsfilme verschmutzt ist.

\subsection{Vorstellung der verwendeten UHV-Apparatur}

Die dieser Abhandlung zugrundeliegenden C-AFM-Ergebnisse wurden an einem unter Ultrahochvakuumbedingungen (UHV) arbeitenden Großgerät erzielt. In der Analysenkammer, welche neben der Rastersondenmikroskopie auch die hier nicht genutzten Optionen der Röntgenphotoelektronenspektroskopie und Augerelektronenspektroskopie beherbergt, können Enddrücke von etwa $10^{-10}$ mbar erreicht werden; eine Schleuse mit etwa $10^{-8}$ mbar erlaubt einen schnellen Proben- und Spitzentransfer ohne das Vakuum brechen zu müssen. ${ }^{\mathrm{e}}$

\footnotetext{
${ }^{\mathrm{d}}$ Ein Sharvinscher Punktkontakt liegt vor, wenn die mittlere freie Weglänge der Ladungsträger größer ist als der Kontaktradius. ${ }^{[129]}$ Der Transport erfolgt dann nicht mehr diffusiv, wie in makroskopischen Kontakten, sondern ballistisch.

eAuf eine autarke, mit dem Analysenrezipienten verbundene Präparationskammer und vakuumtechnische Details wird in dieser Beschreibung nicht eingegangen.
} 

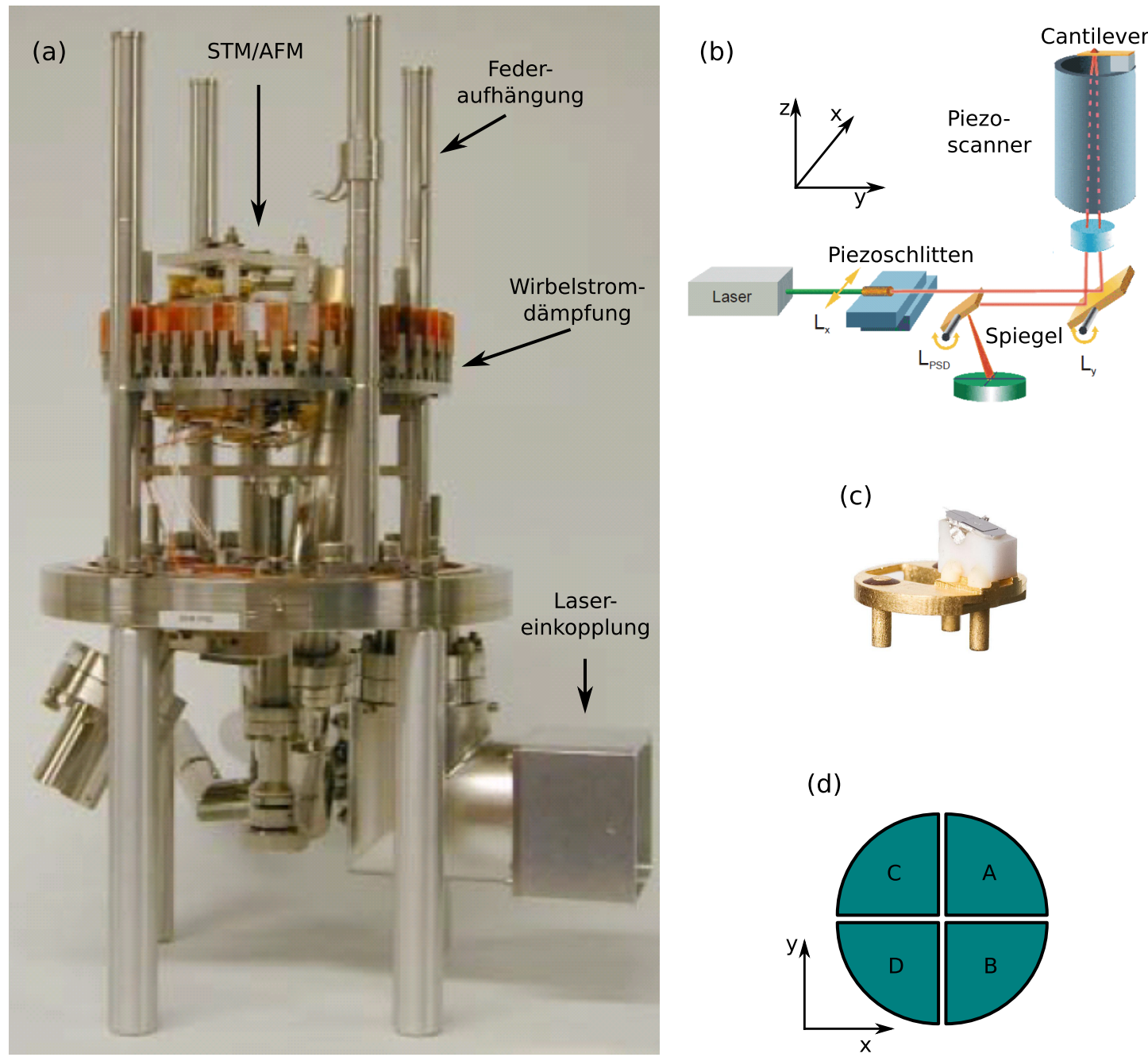

Abbildung 3.5: Aufbau des verwendeten Omicron VT-AFM. (a) zeigt die in der Federsuspension gelagerte Mikroskop-Plattform mit der umgebenden Wirbelstromdämpfung. (b) illustriert den optischen Aufbau zur Detektion der Cantileverbewegungen. Ein auf den Halter aufgeklebter Cantilever ist in (c) zu sehen. (d) zeigt die Benennung der PSD-Sektoren. ((a,b) aus [122], (c) aus [131]) 


\section{Mikroskopeinheit}

Das Herzstück der Anlage ist ein kommerzielles Rastersondenmikroskop, das VariableTemperature AFM (VT-AFM) der Firma Omicron NanoTechnology, Taunusstein. ${ }^{122]}$ Dabei handelt es sich um das in Abb. 3.5 (a) abgebildete kombinierte Rastertunnel- und atomare Kraftmikroskop. Beide Anwendungen bedienen sich einer gemeinsamen Probenund Spitzenaufnahme inklusive der zugehörigen Piezo-Stellelemente. Die Cantilever werden auf einen Keramikkeil (siehe Abb. 3.5 (c)) aufgeklebt, in dessen Mitte sich eine metallische Zuleitung zur Sockelplatte des Sondenträgers verbirgt - durch Verwendung von Leitsilber ist so eine elektrische Kontaktierung möglich. Cantilever- und Tunnelspitzenhalter werden magnetisch auf einer Piezoröhre befestigt, welche durch vier entsprechend positionierte äußere Kontakte in $x$ - und $y$-Richtung einen Scanbereich von $10 \mu \mathrm{m} \times 10 \mu \mathrm{m}$ zugänglich macht. Durch innere Kontakte wird ein vertikales Verfahren um bis zu 1,5 $\mu \mathrm{m}$ ermöglicht. Zur Grobpositionierung des gesamten Messkopfes dient ein piezoelektrischer Trägheitsmotor, er gestattet das Abfahren von Proben bis zu einer Abmessung von $10 \mathrm{~mm} \times 10 \mathrm{~mm}$.

Die Proben können auf einer Reihe verschiedener Halter, welche unterschiedliche Optionen der thermischen Kontaktierung oder auch ein integriertes Heizelement bieten, befestigt werden. Eine elektrische Kontaktierung ist im Falle isolierender Substrate durch eine Leitsilberbrücke oder die Verwendung großflächiger metallischer Klemmkontakte möglich.

Der dem Lichtzeiger-Prinzip folgende optische Aufbau ist in Abb. 3.5 (b) skizziert. Die Auslenkung des Cantilevers wird durch die Richtungsänderung eines daran reflektierten Laserstrahles auf einem positionsempfindlichen Detektor (Position Sensitive Detector, PSD) registriert. Der infrarote Laser der Wellenlänge $\lambda=830 \mathrm{~nm}$ wird faseroptisch außerhalb der UHV-Kammer eingekoppelt. Das Ende des Wellenleiters liegt auf einem Piezoschlitten $\mathrm{L}_{\mathrm{X}}$, wodurch eine Justage des Laserpunktes in $x$-Richtung, also senkrecht zur Cantileverachse, ermöglicht wird. Ein darauf folgender, piezomechanisch drehbarer Spiegel LY gestattet ferner ein Verfahren entlang der Cantileverachse. Auf diese Weise kann das Instrument auch für die außerhalb des Vakuums gegebenenfalls mit leicht verschiedenen relativen Positionen auf die Halter aufgeklebten Cantilever gut so justiert werden, dass der Laserfleck den Cantilever nahe der Spitze trifft und auf Verbiegungen empfindlich reagiert.

Der Laserstrahl tritt von unten in die Piezoröhre ein, verlässt diese durch ein Prismenfenster, wird am Cantilever reflektiert und durchläuft unter der dadurch erfahrenen Verkippung erneut die Piezoröhre zum $y$-Spiegel. ${ }^{f}$ Ein weiterer drehbarer Spiegel LPSD ist schließlich zur Umlenkung und $y$-Justage des Laserpunktes auf dem PSD notwendig, sodass ein am unverbogenen Cantilever reflektierter Strahl den PSD mittig trifft.

\section{Kraftmessung}

Der PSD besteht aus vier Sektoren (siehe Abb. 3.5 (d)), welche eine ihrer Ausleuchtung proportionale Spannung erzeugen. Zieht man von der Summe $U_{\mathrm{A}}+U_{\mathrm{C}}$ der an den oberen Sektoren gemessenen Spannungen die der unteren beiden Messfelder $U_{\mathrm{B}}+U_{\mathrm{D}}$ ab, so erhält

\footnotetext{
${ }^{\mathrm{f}}$ Auf zusätzliche optische Komponenten, die eine Fokussierung auf den Cantilever gewährleisten, aber für die Justage des Gerätes keine Rolle spielen, wird hier nicht eingegangen.
} 
man ein zur Cantileververbiegung und damit an der Spitze wirkenden Normalkraft $F$ proportionales Signal:g

$$
F=a\left[\left(U_{\mathrm{A}}+U_{\mathrm{C}}\right)-\left(U_{\mathrm{B}}+U_{\mathrm{D}}\right)\right]
$$

Im Gegensatz etwa zur interferometrischen Erfassung der Cantileverauslenkung liegt also zunächst keine direkte quantitative Information über die Bewegung der Cantileverspitze vor. Für eine absolute Kraftmessung aus der Spannungsdifferenz am PSD muss deshalb die folgende Kalibrierung zur Bestimmung der Proportionalitätskonstanten $a$ durchgeführt werden: Das Spannungssignal wird im elastischen Kontaktregime einer Kraft-Abstands-Kurve linear angefittet. Dabei wird von einer im Vergleich zur Cantileververbiegung vernachlässigbaren Verformung der Probe ausgegangen - eine Bedingung, die für die sehr steifen Manganate gut erfüllt ist und keine zusätzlichen Kalibrierungsmessungen auf härteren Proben erforderlich macht. Die vom Hersteller meist gemäß Gleichung 3.5 geometrisch abgeschätzte Federkonstante des Cantilevers erlaubt nun unter Annahme der Gültigkeit des Hookeschen Gesetzes $F=-k z$ eine Umrechnung der Spannungsdifferenzen in Wechselwirkungskräfte.

\section{Schwingungsdämpfung}

Eine weitestmögliche Abdämpfung aller extern einkoppelnden Schwingungen ist bei der Rastersondenmikroskopie von essentieller Bedeutung. Neben einstreuenden elektrischen Störsignalen kommt hier auch mechanischen Schwingungen aufgrund der sehr empfindlichen topographischen Messungen besondere Bedeutung zu: Auch kleinste Verschiebungen zwischen Spitze und Probe müssen vermieden werden. Das VT-AFM verfügt dazu über eine Federaufhängung des gesamten Mikroskopaufbaus, die Resonanzfrequenz beträgt $2 \mathrm{~Hz}$. Am Rand der Basisplatte befinden sich außerdem Kupferbleche, welche zwischen außen angebrachten, starken Permanentmagneten gelagert sind. Bewegungen des Mikroskopes werden so durch die Induktion von Wirbelströmen rasch abgedämpft. Bei der Durchführung von AFM-Messungen muss ferner gegebenenfalls auch dem Einfallen von Raumlichtanteilen auf den PSD durch eine Verdunklung der Sichtfenster der UHV-Anlage vorgebeugt werden.

\section{Temperaturbereich}

Das Mikroskop verfügt ferner über einen thermisch angekoppelten Durchflusskryostaten, welcher mit flüssigem Stickstoff oder Helium betrieben werden kann. Ein integriertes Heizelement erlaubt so Endtemperaturen bis zu $25 \mathrm{~K}$ einzustellen. Durch die Verwendung von Probenhaltern mit einem eingebauten Heizer aus pyrolytischem Bornitrid können ferner Temperaturen bis zu $750 \mathrm{~K}$ realisiert werden. Hochohmige Proben können außerdem durch direkte Heizströme auf noch höhere Temperaturen gebracht werden.

${ }^{g}$ Durch analoge Differenzbildung der Signale der linken und rechten PSD-Sektoren können laterale Reibungskräfte während des Scanvorgangs, die eine Torsion des Cantilevers bewirken, untersucht werden. 


\section{Ansteuerung und Auswertung}

Die rechnerbasierte Ansteuerung der Mikroskopelektronik geschieht mit der zugehörigen Software SCALA PRO 5.0 ${ }^{[132]}$. Sie verfügt über eine integrierte Skriptsprache, welche die Erzeugung komplexer Makros erlaubt und für die im Rahmen dieser Arbeit durchgeführten Experimente intensiv genutzt wurde. Zur Auswertung wurden neben der ScALA-Software vor allem eigens erstellte MATLAB-Algorithmen verwendet.

\section{Elektrische Messungen}

Die Verwendung leitfähig beschichteter Cantilever bei der simultanen Durchführung von topographischen und elektrischen Messungen geschieht ähnlich wie im STM-Betrieb. Es wird dabei auch im AFM-Betrieb der STM-Vorverstärker - sowohl zum Anlegen der Spannung von bis zu $\pm 10 \mathrm{~V}$ als auch zur Erfassung des Stromes - verwendet. Hierbei steht ein Messbereich von maximal $\pm 50 \mathrm{nA}$ zur Verfügung. Dabei liegt die Probe stets auf geerdetem Potential; die Polarität der in dieser Arbeit angegebenen Spannungen bezieht sich also immer auf die Spitze.

Die im Rahmen dieser Arbeit verwendeten Cantilever sind aus Silicium gefertigt und mit einer Platinbedampfung, zum Teil auf einer Titan-Adhäsionsschicht, versehen. Laut Herstellerangaben von MikroMasch, Tallinn (Estland) beträgt die Dicke der Beschichtung etwa $30 \mathrm{~nm}$ und der resultierende sphärische Spitzenradius $30 \ldots 40 \mathrm{~nm}$. Dieser Wert ist vergleichsweise groß, sodass keine hochaufgelösten topographischen Aufnahmen möglich sind. Außerdem haben einige Fabrikate eine zusätzliche Aluminiumbeschichtung auf der Rückseite des Cantilevers, um die Reflektivität zu verbessern. Die Federkonstante aller verwendeten Proben lag bei $0,15 \mathrm{~N} / \mathrm{m}$.

\subsection{Abschätzung des minimalen Kontaktradius}

An dieser Stelle soll mittels des Hertzschen Kontaktmodells ${ }^{[120]}$ eine grobe Abschätzung für die Kontaktdimensionen gewonnen werden. Streng genommen befinden sich die experimentellen Verhältnisse außerhalb des Gültigkeitsbereiches der Hertzschen Kontaktmechanik. Diese beschreibt nämlich die elastische Verformung einer sphärischen und vergleichsweise weichen Spitze, wenn sie unter Abwesenheit von Adhäsionskräften auf eine idealerweise unendlich harte und ebene Unterlage gedrückt wird.

Die vergleichbaren elastischen Moduli von Cantileverspitze und Manganat lassen hingegen auch eine Verformung der Probe erwarten, wodurch ein größerer Kontaktradius zu erwarten ist. Wir verzichten dennoch auf eine aufwendigere Analyse zum Beispiel in Kombination mit der Sneddonschen Theorie, welche den Deformationen einer weichen Probe durch die harte Spitze gewidmet ist, oder erweiterten Kontaktmodellen, die Adhäsionskräfte beinhalten. ${ }^{\mathrm{h}}$ Auch diese würden nur zu einer Vergrößerung der Kontaktfläche führen, weshalb wir die folgende Näherung im Rahmen der Hertzschen Kontaktmechanik als eine untere Grenze für die Kontaktfläche ansehen können. Als minimalen Kontaktradius a nehmen wir also an:

$$
a=\sqrt[3]{(R F / K)}
$$

\footnotetext{
${ }^{\mathrm{h}}$ Diese Vereinfachung ist in der C-AFM-Literatur durchaus gängig, siehe zum Beispiel [130].
} 
Hierbei ist $R=35 \mathrm{~nm}$ laut Herstellerangaben der sphärische Radius der verwendeten Sonden. Als typische Andruckkraft setzen wir hier ferner $F=5 \mathrm{nN}$ an, während $K$ den reduzierten Elastischen Modul des aus Spitze und Probe gebildeten mechanischen Systems kennzeichnet. Dieses ist gegeben durch:[120]

$$
K=\frac{4}{3}\left[\left(1-\nu_{t}^{2}\right) / E_{t}+\left(1-\nu_{s}^{2}\right) / E_{s}\right]^{-1} .
$$

Wir rechnen für die Sonde mit einem Elastizitätsmodul von $E_{t}=169 \mathrm{GPa}$ (nach Herstellerangaben) und einer Poissonzahl von $\nu_{t} \approx 0,27$, letztere gemäß Referenz [133]. Es wird dabei die metallische Beschichtung mit Titan und Platin außer Acht gelassen. Als grobe Näherung erscheint dies zulässig, da auch hier ähnliche Steifigkeiten erwartet werden können und eine detaillierte Untersuchung der elastischen Eigenschaften dieses mehrlagigen Systems weit über den Umfang der vorliegenden Arbeit hinausginge.

Für die Probe werden exemplarisch die Werte von unverspanntem LCMO, $E_{s}=135 \mathrm{GPa}$ sowie $\nu_{s}=0,35^{[134]}$, angenommen und der anisotrope Charakter der elastischen Konstanten vernachlässigt. Wir gelangen so zu dem minimal denkbaren Kontaktradius von:

$$
a \geq 1,1 \mathrm{~nm} .
$$

Auch wenn die tatsächliche Ausdehnung des mechanischen und somit auch elektrischen Kontaktes diese abgeschätzte untere Grenze mit Sicherheit deutlich übertrifft, wird sich dieser Wert in späteren Abschnitten als nützlich erweisen um eine obere Schranke für auftretende Stromdichten und den dadurch verursachten Jouleschen Wärmeeintrag zu erhalten. 


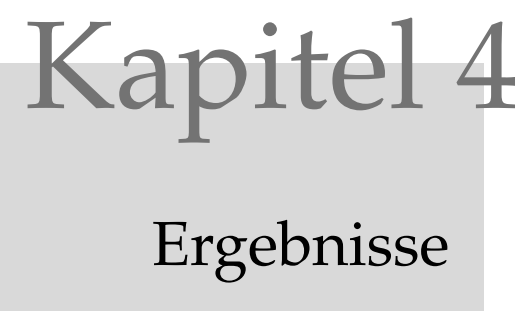

Dieses Kapitel beschreibt die gewonnenen experimentellen Ergebnisse zum bipolaren Widerstandsschalten von Manganatoberflächen. Die ersten drei Abschnitte sind zunächst der Charakterisierung der untersuchten Proben gewidmet. Dabei werden exemplarisch an einer $\mathrm{La}_{0,8} \mathrm{Ca}_{0,2} \mathrm{MnO}_{3}: \mathrm{MgO}$-Probe die kristalline Struktur und Qualität, die elektrische Leitfähigkeit mit typischem kolossalen Magnetowiderstandsverhalten und die Oberflächenbeschaffenheit knapp dargestellt.

Diese Probe wurde ausgewählt, da die qualitativ besten Resultate zum Widerstandsschalten an ihr gewonnen wurden. Die gezeigten Struktur- und Oberflächeneigenschaften sind beispielhaft auf alle hier untersuchten Systeme übertragbar und die jeweilige dotierungstypische globale Temperatur- und Magnetfeld-Abhängigkeit des makroskopischen elektrischen Widerstands spiegelt sich nicht wesentlich in den Eigenschaften des auf der Nanometerskala untersuchten Schalteffektes wider. Eine ausführliche Darstellung der makroskopischen Eigenschaften aller weiteren betrachteten Manganatproben ist daher für das Verständnis der elektrischen Experimente nicht erforderlich.

Das Widerstandsschalten wird einleitend zunächst nur allgemein und auf die elektrischen Eigenschaften beschränkt klassifiziert. Es handelt sich dabei allerdings schon um C-AFMExperimente; es wird also das lokale Widerstandsverhalten der Probenoberfläche untersucht.

Es folgt eine Vorstellung der räumlichen Eigenschaften anhand aufgezeichneter Stromkarten, deren instruktiver Charakter vor allem einem intuitiven Zugang dienlich ist und ein qualitatives Verständnis vermittelt. Dabei wird auch auf die in den topographischen Aufzeichnungen ersichtlichen, manchmal mit dem elektrischen Schalten einhergehenden Oberflächenmodifikationen eingegangen. Eine Analyse des Einflusses von verschieden starken und langen Schaltpulsen auf das Domänenbild vermittelt dann einen ersten Eindruck von den Wachstumsprozessen metallischer Bereiche. Untersuchungen der selbstständigen Rückbildung metallischer Domänen nach dem Spannungspuls und des abwechselnden Wachsens und Relaxierens im Zuge von Pulsfolgen runden das Bild von der Ausbreitung der Leitfähigkeitsänderung ab. 
Probeneffekte wurden in der vorliegenden Arbeit nicht systematisch - etwa durch eine Variation der Dotierung oder die Wahl anderer Substrate - untersucht. Ein Abschnitt widmet sich jedoch solchen Resultaten, welche vom allgemeinen, für alle untersuchten Proben ähnlichen Schaltverhalten abweichen. Darin wird zunächst der drastische Effekt einer Modifikation durch zwei zusätzliche LMO-Lagen auf die Oberflächenleitfähigkeit von LCMO dargestellt. Außerdem wird auf den Befund fester Widerstandswerte - welcher bisher nur für eine LSMO-Probe vorliegt - und ihre Korrelation mit der Topographie eingegangen. Das Kapitel schließt mit einer kurzen Zusammenfassung der wichtigsten experimentellen Ergebnisse.

\subsection{Struktur}
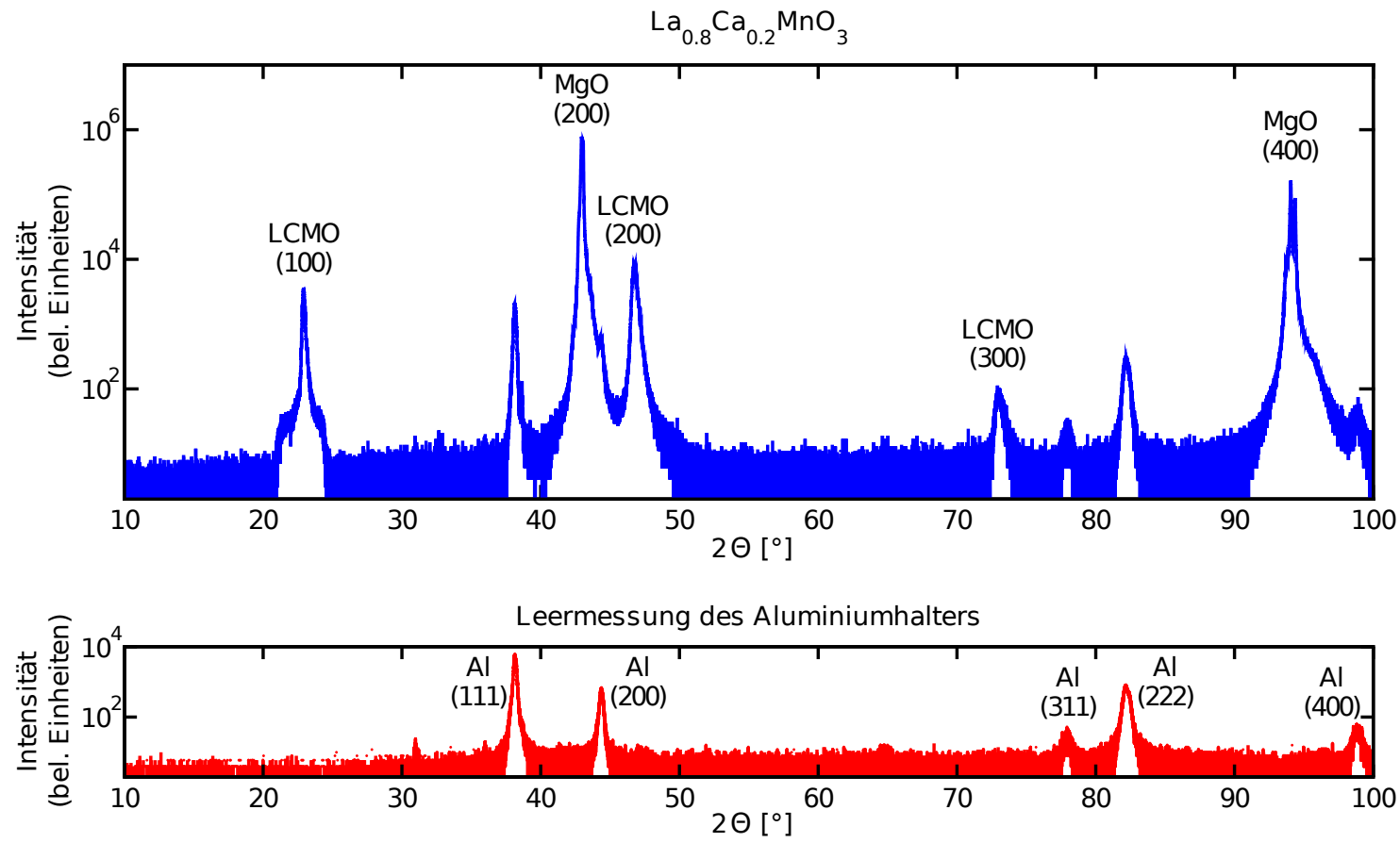

Abbildung 4.1: Röntgendiffraktogramm der $\mathrm{La}_{0,8} \mathrm{Ca}_{0,2} \mathrm{MnO}_{3}: \mathrm{MgO}-$ Probe mit indizierten Substrat- und Manganat-Reflexen (oben). Dem gegenübergestellt wurde eine Leermessung des Aluminiumhalters (unten).

Eine röntgendiffraktometrische Weitwinkelaufnahme der $\mathrm{La}_{0,8} \mathrm{Ca}_{0,2}: \mathrm{MnO}_{3}$-Schicht ist in Abb. $4.1 \mathrm{zu}$ sehen. Eine Vergleichsmessung zeigt zudem die Aluminium-Reflexe des Probenhalters, welche im Falle der LCMO-Schicht nicht indiziert sind. Neben den dominanten MgO-Reflexen des Substrats sind ausschließlich die (h00)-Peaks vom LCMO zu sehen. Im Vergleich zu früheren Untersuchungen ${ }^{[109]}$ liegt demnach wahrscheinlich epitaktisches Filmwachstum einer chemisch einphasigen Schicht vor. Die gemäß der Braggschen Reflexionsbedingung bestimmte Gitterkonstante beträgt $a=0,388 \mathrm{~nm}$. 
(a) $\mathbf{R}(\mathbf{T})$-Verlauf

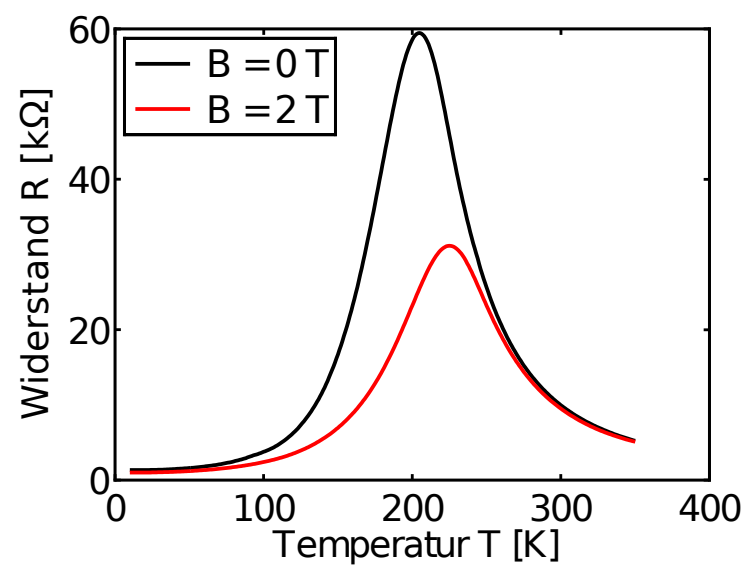

(b) I-U-Charakteristika

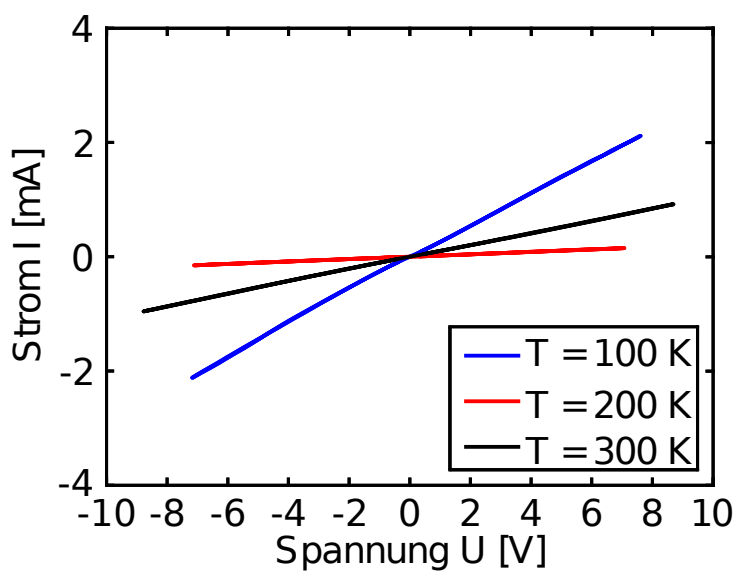

Abbildung 4.2: Makroskopische elektrische Eigenschaften, bestimmt nach der VierpunktMethode. (a) zeigt die Temperaturabhängigkeit des elektrischen Widerstands bei $B=0 \mathrm{~T}$ und $B=2 \mathrm{~T}$ mit typischen CMR-Eigenschaften, (b) das Ohmsche Verhalten bei ausgewählten Temperaturen.

Zur Schichtdickenbestimmung wurde außerdem eine Kleinwinkelanalyse (nicht gezeigt) durchgeführt, für diese Probe ergibt sich ein Wert von $d \approx 40 \mathrm{~nm}$.

\subsection{Makroskopische elektronische Eigenschaften}

Es wurden globale Widerstandsmessungen mit Leitsilberkontakten von knapp einem Millimeter Durchmesser und Kontaktabständen von mehreren Millimetern durchgeführt. Um Einflüsse der Zuleitungen und Kontaktierung möglichst gering zu halten, wurde der Widerstand nach der Vierpunktmethode bestimmt.

Der temperaturabhängige Widerstandsverlauf (Abb. 4.2) zeigt den für LCMO typischen Metall-Isolator-Übergang mit einem Widerstandsmaximum bei $T_{\mathrm{MI}}=205 \mathrm{~K}$, beziehungsweise einem stärksten Widerstandsanstieg bei $T=180 \mathrm{~K}$. Die Lage des Maximums erlaubt, in Ergänzung zu der oben bestimmten Gitterkonstante von $a=0,388 \mathrm{~nm}$, den Rückschluss auf eine relative Calciumdotierung von $x=0,2 \cdot{ }^{a}$ In einem angelegten Magnetfeld der Flussdichte $B=2 \mathrm{~T}$ findet eine deutliche Widerstandsabnahme von bis zu $65 \%$ statt, es sind also die Charakteristika des kolossalen Magnetowiderstandseffektes erkennbar.

Die in Abb. 4.2 dargestellten Strom-Spannungs-Kennlinien nahe $T_{\mathrm{MI}}=205 \mathrm{~K}$ sowie fern des Überganges zeigen - im Rahmen des untersuchten kleinen Spannungsintervalls von $|U|<10 \mathrm{~V}$ - alle lineare Spannungsabhängigkeit, das heißt Ohmsches Verhalten. Eventuelle Nichtlinearitäten und Asymmetrien erscheinen in diesen globalen Messungen vernachlässigbar und insbesondere ein Schalten des Widerstandes, also Stufen im $I-U$ Verlauf, sind nicht feststellbar.

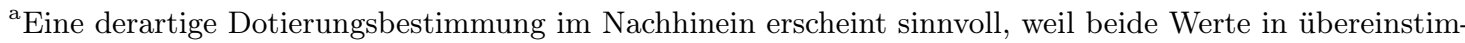
mender Weise von denen des optimal dotierten $\mathrm{La}_{0,7} \mathrm{Ca}_{0,3} \mathrm{MnO}_{3}$, dessen Stoffmengenverhältnis bei der Probenpräparation an sich abgewogen worden war, abweichen. ${ }^{[135]}$ 


\subsection{Oberflächenmorphologie}

Im Folgenden wird die Gestalt der Oberflächen der untersuchten Proben anhand einer charakteristischen STM-Messung besprochen. Die C-AFM-Methodik erlaubt zwar auch die Erfassung topographischer Daten, ist in ihrer Auflösung jedoch dem STM wegen des deutlich vergrößerten Spitzenradius eines beschichteten Cantilevers $(r=35 \mathrm{~nm})$ deutlich unterlegen.

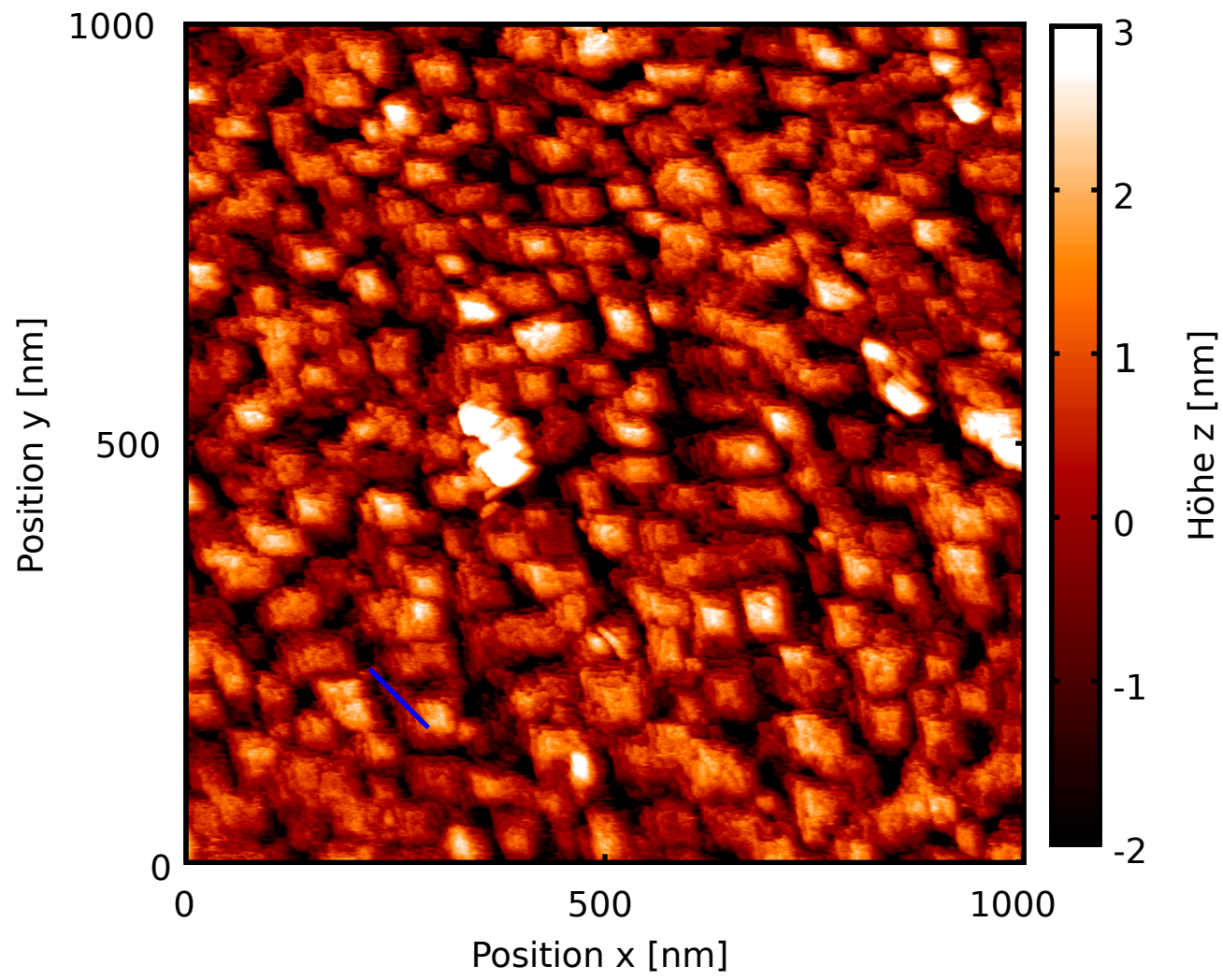

Abbildung 4.3: STM-Topographie, gewonnen mit $U_{\mathrm{G}}=0,7 \mathrm{~nm}$ und $I=0,2 \mathrm{nA}$. Angesichts der geringen Rauigkeit sind atomare Terrassen erkennbar. Die blaue Linie kennzeichnet den Pfad des in Abb. 4.4 gezeigten Höhenprofils.

Da wegen des erforderlichen Spitzenwechsels, der auch ein weiträumiges laterales Verfahren der Piezos erfordert, keine STM-Bilder an denselben Stellen der in den späteren Abschnitten gezeigten C-AFM-Ergebnisse gewonnen werden können, haben die hier gezeigten Topographien ausschließlich exemplarischen Charakter. Natürlich wurde bei den in späteren Abschnitten gezeigten kraftmikroskopischen Experimenten stets darauf geachtet, Probenregionen mit deutlich erhöhter lokaler Rauigkeit zu meiden.

Abb. 4.3 demonstriert das Vorliegen einer lagenweise gewachsenen LCMO-Schicht von geringer Rauigkeit. Auf der Längenskala der Bildabmessungen von $1000 \times 1000 \mathrm{~nm}^{2}$ beträgt die Rauigkeit nur $R M S=0,9 \mathrm{~nm}$, was von der guten kristallinen Qualität der Schicht zeugt. Terrassenartige Strukturen sind deutlich erkennbar, die Stufenhöhe beträgt dabei - wie 
im nachfolgend ergänzten Linienprofil (Abb. 4.4) verdeutlicht - stets Vielfache der Dicke einer ganzen Monolage.

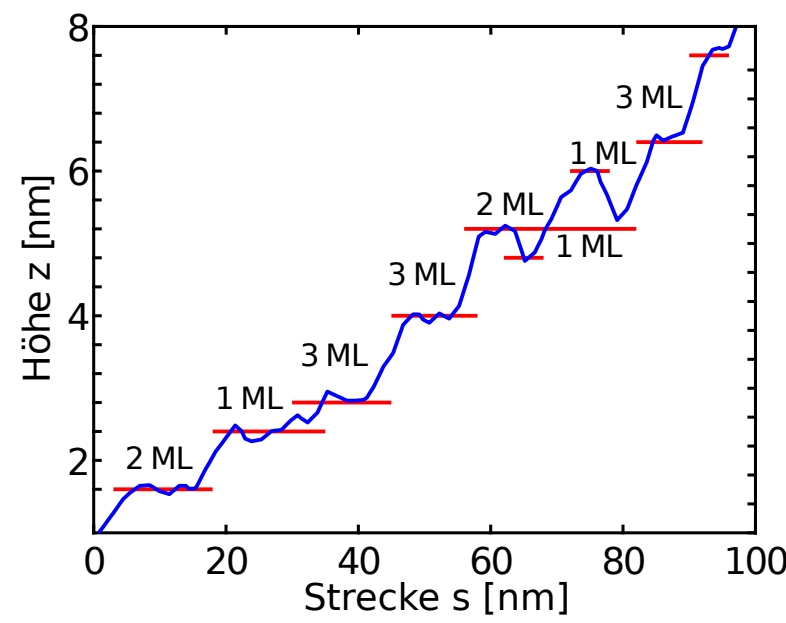

Abbildung 4.4: Höhenprofil zu Abb. 4.3. Die Terrassen und ihre Abstände - Vielfache einer Monolagendicke - wurden gekennzeichnet.

\subsection{Resistives Schalten: Charakterisierung des Effektes}

Nach der in den vorherigen Abschnitten erfolgten Charakterisierung des untersuchten Systems widmen wir uns nun den für diese Abhandlung zentralen Ergebnissen. Zu Beginn beschränken wir uns dabei ausschließlich auf eine Darstellung des elektrischen Widerstandsverhaltens, erst in den anschließenden Passagen werden orts- und zeitaufgelöste Messungen, Temperaturabhängigkeiten und der Einfluss modifizierter Probenoberflächen behandelt. Das Schaltphänomen, welches so nur mit der C-AFM-Methode, nicht aber unter makroskopischer Kontaktierung beobachtet wurde, weist folgende wesentliche Merkmale auf:

- Der Ausgangszustand der nicht modifizierten Manganatoberflächen erschien in allen Experimenten hochisolierend: Es wurden bei Spannungen von bis zu einem Volt meist nur sehr geringe Ströme oder ausschließlich Rauschen $<10$ pA gemessen. Dies ist insbesondere bemerkenswert, da Rastertunnelmikroskopie an den gleichen Manganatproben mit Gapspannungen $U_{\mathrm{G}}=0,7 \mathrm{~V}$ und Tunnelströmen von $I=0,2 \mathrm{nA}$ betrieben wurde. Die hier gezeigten Messungen sind hingegen alle im mechanischen Kontakt zwischen Cantileverspitze und Probe erfolgt und man würde intuitiv einen höheren Leitwert erwarten als im Tunnelkontakt.

- Das Schalten zum niederohmigen Zustand (LRS, low resistive state) wird stets nur durch Anlegen einer positiven Spannung an die AFM-Spitze initiiert. Negative Spannungen zeigen keinen Effekt. Der Schaltvorgang ist also bipolar.

- Es ist stets eine Schwellspannung erforderlich, um ein Widerstandsschalten auslösen zu können. Unterhalb dieser charakteristischen Amplitude wurde auch nach 
sehr viel längeren Pulsen keine Widerstandserniedrigung festgestellt. Die kleinsten Schwellspannungen betrugen dabei etwa $U_{\mathrm{c}} \approx 3 \mathrm{~V}$, oftmals waren jedoch auch stärkere Pulse von bis zu $U_{\mathrm{c}}=9 \mathrm{~V}$ nötig.

- Der niederohmige Zustand bleibt oftmals erhalten, auch wenn die Schwellspannung nicht mehr anliegt und kann später mit Spannungen $U \ll U_{\mathrm{c}}$ nachgewiesen werden. Die Widerstandsänderung erfolgt also häufig - nicht immer - remanent. Es sei darauf hingewiesen, dass die Langzeitstabilität eines lokal veränderten Widerstandes mit dieser Methode nicht untersucht werden kann; es war jedoch in einigen Messungen auch über Stunden kein Abklingen des leitfähigen Zustandes erkennbar.

- Das Anlegen einer negativen Spannung in etwa des gleichen kritischen Betrags $U_{\mathrm{c}}$ versetzt die Probe wieder in einen hochohmigen Zustand (HRS, high resistive state). ${ }^{\mathrm{b}}$ Dieser kann jedoch noch immer deutlich leitfähiger sein als der Ausgangszustand. Zwischen LRS und HRS lässt sich wiederholt wechseln, der Schaltprozess ist reversibel.

- Die Messungen zeigen große statistische Schwankungen: Es konnte zwar in der überwiegenden Zahl der Messungen das gleiche qualitative Verhalten beobachtet werden, jedoch schwanken beispielsweise die Widerstandswerte und Schaltspannungen sogar in Abhängigkeit von der untersuchten Oberflächenregion einer einzigen Probe oftmals beträchtlich. Zusätzliche instrumentelle Unsicherheiten wie das Altern und die individuellen Kontakteigenschaften der leitfähigen Sonden sowie sich im Experiment ereignende Modifikationen der Probenoberfläche machen dagegen exakte quantitative Aussagen nahezu unmöglich. Die Angabe konkreter Werte soll hier also vor allem ein Gefühl für die relevanten Größenordnungen vermitteln.

Abb. 4.5 illustriert die stark unterschiedlichen Widerstandszustände anhand von $I-U$ Kennlinien. Dazu wurde auf einer Oberflächenregion von $500 \times 500 \mathrm{~nm}^{2}$ ein Raster von $11 \times 11$ Spektren aufgenommen. Jedes einzelne bestand aus insgesamt sieben Spannungsrampen über das Intervall $-1 \ldots 1 \mathrm{~V}$. Zwischen diesen $I$ - $U$-Kennlinien wurden $20 \mathrm{~ms}$ lange Spannungspulse der Stärke $\pm 4 \mathrm{~V}$ angelegt - abwechselnd positiv und negativ. Es werden zunächst nur die ersten drei $I$ - $U$-Kurven untersucht, also die des Ausgangszustandes, nach dem ersten Schalten in den LRS und dem ersten Zurückschalten in den HRS. Dabei wurde über 60 erfolgreiche Schaltprozesse gemittelt.

Während im hochisolierenden Ausgangszustand praktisch nur eine horizontale Kennlinie vorliegt, zeigt der LRS einen so starken Anstieg, dass die Ströme den instrumentell erfassbaren Bereich von $\pm 50 \mathrm{nA}$ bereits bei wenigen hundert Millivolt überschreiten und der Vorverstärker in die Sättigung läuft. Die $I$ - $U$-Charakteristik des HRS ist demgegenüber deutlich flacher. Aufgrund der apparativen Begrenzung auf $\pm 50 \mathrm{nA}$ ist es nicht möglich, eine genaue Analyse des Sprungverhaltens um $U_{\mathrm{c}}$ durchzuführen: Unmittelbar nach dem Überschreiten der Schwellspannung verlassen die Stromwerte den Messbereich und der

\footnotetext{
${ }^{\mathrm{b}}$ Nach Einführung der Bezeichnungen LRS und HRS werden im Folgenden die Begriffe des metallischen beziehungsweise isolierenden Zustandes damit synonym verwendet. Hiermit soll jedoch nicht die Annahme eines tatsächlich metallischen Transportmechanismus itineranter Ladungsträger zum Ausdruck gebracht werden; die Formulierung bezeichnet hier lediglich die in Relation zum HRS stark erhöhte Leitfähigkeit.
} 
(a) I-U-Kennlinien

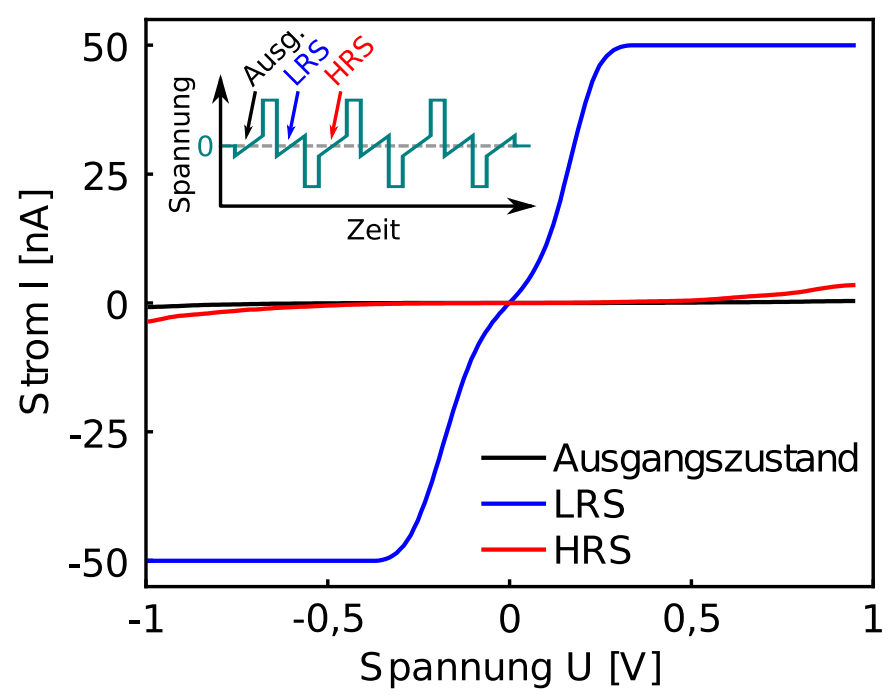

(b) Kennlinien (vergrößert)

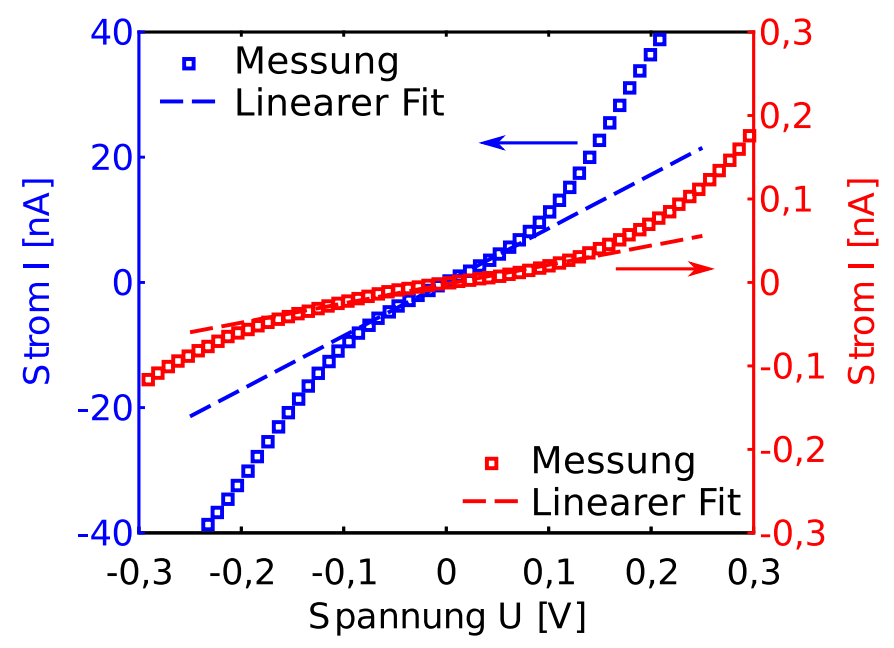

Abbildung 4.5: Auf der untersuchten Region wurde ein Raster von $11 \times 11$ Kennlinien aufgenommen. Von diesen 121 Messungen erreichten 60 Kennlinien nach dem ersten positiven Spannungspuls maximale Stromwerte $I \geq 50 \mathrm{nA}$. Nur diese erfolgreichen Schaltexperimente wurden gemittelt und die Kennlinien geringfügig geglättet (a). Der Einschub illustriert den angelegten Spannungsverlauf. Der vergrößerte Ausschnitt (b) zeigt die zur Widerstandsbestimmung angefitteten Geraden bei kleinen Spannungen und verdeutlicht den anschließenden nichtlinearen Verlauf für höhere Spannungen. Die Schaltpulse waren $\pm 4 \mathrm{~V}$ stark und $20 \mathrm{~ms}$ lang. 
weitere Verlauf der Kennlinie ist nicht mehr zugänglich - man kann also nicht erkennen, ob an der Schaltstufe wirklich ein abrupter Übergang zur LRS-Kennlinie stattfindet.

Die inversen Steigungen linearer Fits ${ }^{\mathrm{c}}$ im Bereich $\pm 50 \mathrm{mV}$ bzw. $\pm 150 \mathrm{mV}$ (siehe Abb. 4.5 (b)) ergeben für diese Messung Widerstände von $R_{\mathrm{LRS}} \approx 12 \mathrm{M} \Omega$ und $R_{\mathrm{HRS}} \approx 4,3 \mathrm{G} \Omega$. In Analogie zu anderen Schaltphänomenen kann die Effektstärke nun angegeben werden als:

$$
\frac{R_{\mathrm{HRS}}-R_{\mathrm{LRS}}}{R_{\mathrm{LRS}}} \approx 37.000 \% .
$$

Im HRS und besonders im LRS ist für größere Spannungen ferner ein Abknicken der Kennlinien hin zu größeren Leitfähigkeiten erkennbar - beide Widerstandszustände zeigen hier also nichtohmsches Verhalten.

Das vollständige Experiment, bestehend also aus sechs abwechselnd positiven und negativen Spannungspulsen und dazwischen jeweils einer Kennlinie ist in Abb. 4.6 dargestellt. Das Schalten ist demnach prinzipiell wiederholbar, es ist jedoch eine Alterung erkennbar: Der hochohmige Zustand zeigt dabei mit fortschreitender Anzahl von Schaltzyklen, sieht man von dem drastischen Unterschied zum Ausgangszustand ab, keine eindeutige Tendenz, während die Leitfähigkeit des metallischen Zustandes sukzessive sinkt.

(a) Wiederholtes Hin- und Herschalten

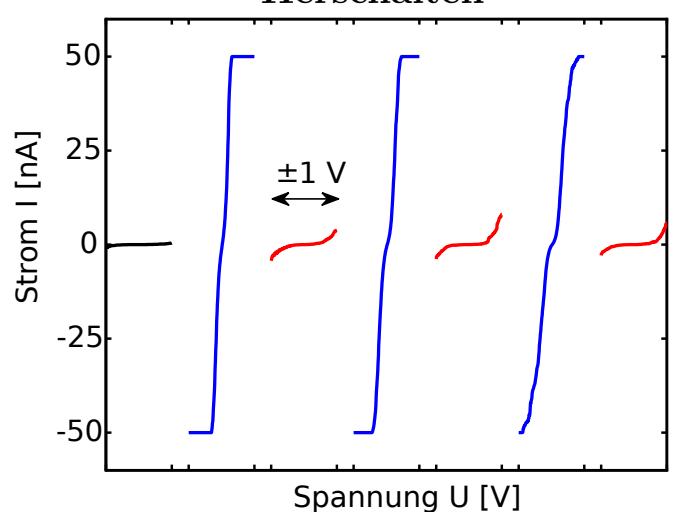

(b) Ermattung mit fortschreitender Pulszahl

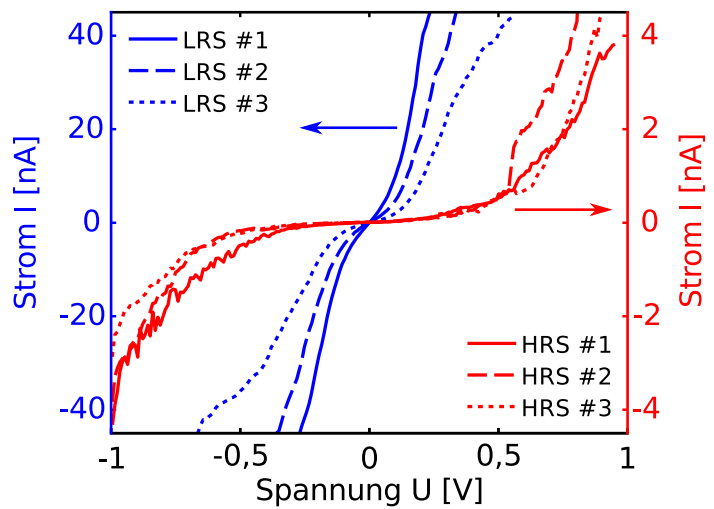

Abbildung 4.6: $I-U$-Kennlinien nach mehreren Schaltzyklen. Es wurden 54 der in Abb. 4.5 gezeigten Messungen ausgewählt, in denen jeweils drei vollständige Schaltzyklen durchlaufen werden konnten (a). In der Vergrößerung (b) erkennt man eine Ermattung des LRS. Die Schaltpulse waren je $\pm 4 \mathrm{~V}$ stark und $20 \mathrm{~ms}$ lang.

\footnotetext{
${ }^{c}$ Der Verlauf der in Abb. 4.5 und Abb. 4.6 gezeigten Kennlinien entspricht zwar qualitativ den Erwartungen im Modell einer asymmetrischen Tunnelbarriere nach Brinkman et al. ${ }^{[136]}$ Auf entsprechende Fitkurven wird hier jedoch verzichtet, weil man keine realistisch erscheinenden Parametersätze erhält Es fehlt dafür nicht nur eine Kenntnis der genauen Kontaktabmessungen; wahrscheinlich greift auch das Bild einer einfachen Tunnelbarriere in diesem Falle zu kurz.
} 


\subsection{Ortsaufgelöste Messungen}

Wir wenden uns nun den ortsaufgelösten Messungen zu, das heißt Stromkarten und Topographien, die nach oder während der Durchführung von Pulsexperimenten aufgenommen wurden. Auf diese Weise können die Ausbreitung der Widerstandsänderung auf der Oberfläche studiert und durch systematische Variation der Pulsparameter bereits erste Rückschlüsse auf deren Einfluss auf das Phasenwachstum der metallischen Domänen gezogen werden.

Es ist dabei zu beachten, dass durch die verzögerte Aufnahme der Stromkarten nur der vergleichsweise langzeitstabile Anteil der induzierten Änderungen abgebildet wird; eventuell sich vor Vollendung der Messung rückbildende metallische Bereiche werden nicht - oder zumindest nicht in ihrer maximalen Ausdehnung - erfasst. Die schnelle Scanrichtung ist in allen gezeigten Bildern von unten nach oben dargestellt. Veränderungen der lokalen Leitfähigkeit, die sich erst im Verlaufe der Aufzeichnung eines Bildes ereignen, sind daher in den darunter liegenden Bildbereichen noch nicht erfasst. Basierend auf den in diesem Abschnitt gezeigten Ergebnissen sind also nur unter vorausgesetzter Remanenz der abgebildeten resistiven Phasenseparation Aussagen über die Domänenausdehnung möglich.

\subsubsection{Stromkarten elektrisch induzierter Widerstandsänderungen}

Einführend sollen nun einige Beispiele aus Pulsversuchen resultierender Anordnungen metallischer und isolierender Oberflächenregionen vorgestellt werden. Die im Folgenden behandelten Stromkarten zeigen den Leitfähigkeitszustand der Oberfläche nach Einwirkung von Spannungen oberhalb des Schwellwertes $U_{\mathrm{c}}$. Dabei wurde vor Beginn eines jeden Pulsexperimentes der stark isolierende Ausgangzustand durch Aufnahme einer Stromkarte bei geringer Spannung sichergestellt (nicht abgebildet).

(a) Stromkarte

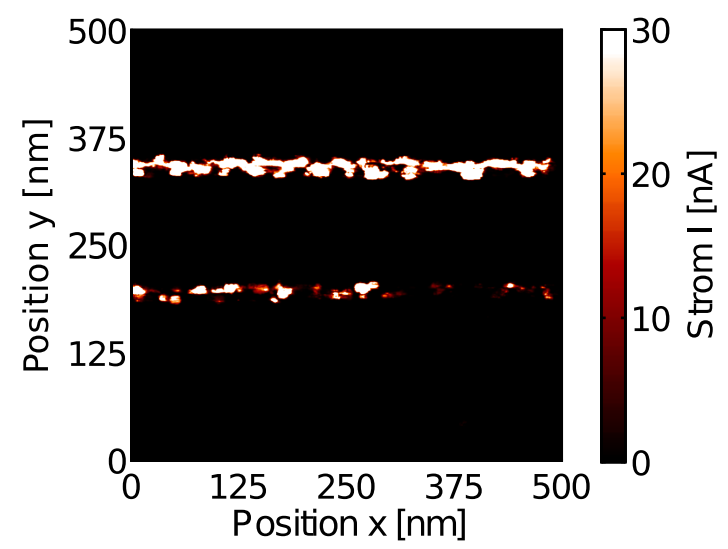

(b) Topographie

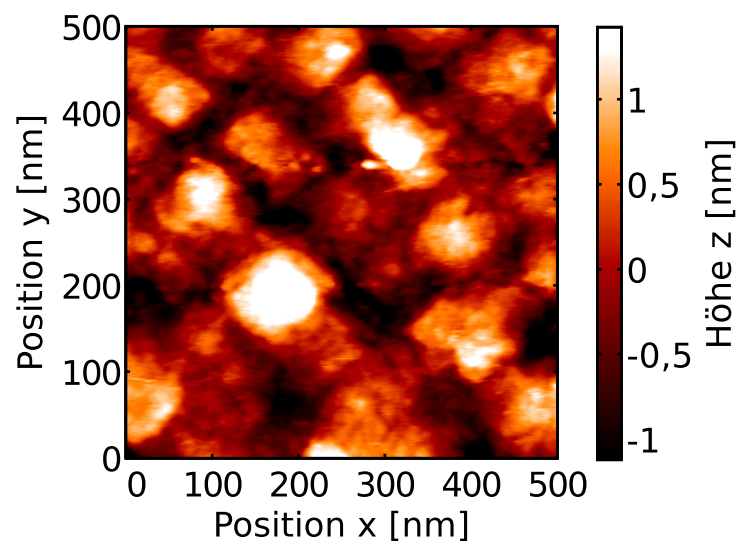

Abbildung 4.7: Bei $U=100 \mathrm{mV}$ aufgenommene Stromkarte (a) einer $\mathrm{La}_{0,85} \mathrm{Sr}_{0,15} \mathrm{MnO}_{3^{-}}$ Schicht mit zugehöriger Topographie (b). In einer Vormessung wurde jeweils für einige Linien die Spitzenspannung auf $U=3 \mathrm{~V}$ erhöht, wodurch eine Phasenseparation in leitende und isolierende Bereiche induziert wurde. 
(a) Stromkarte

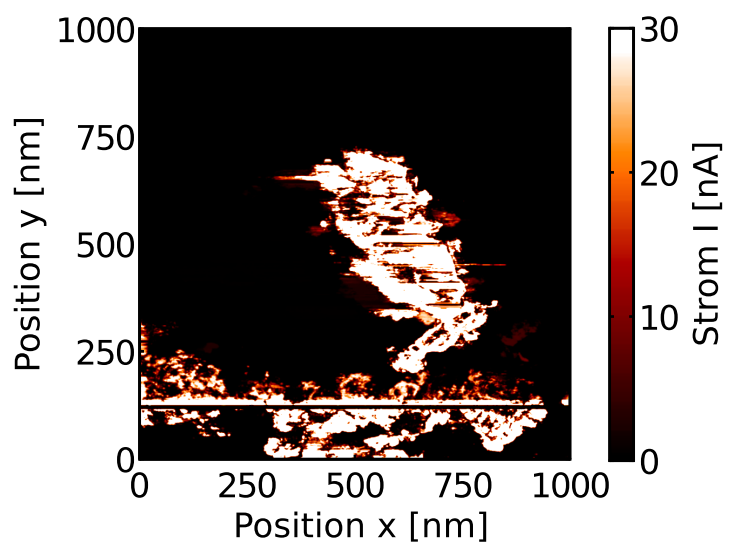

(b) Topographie

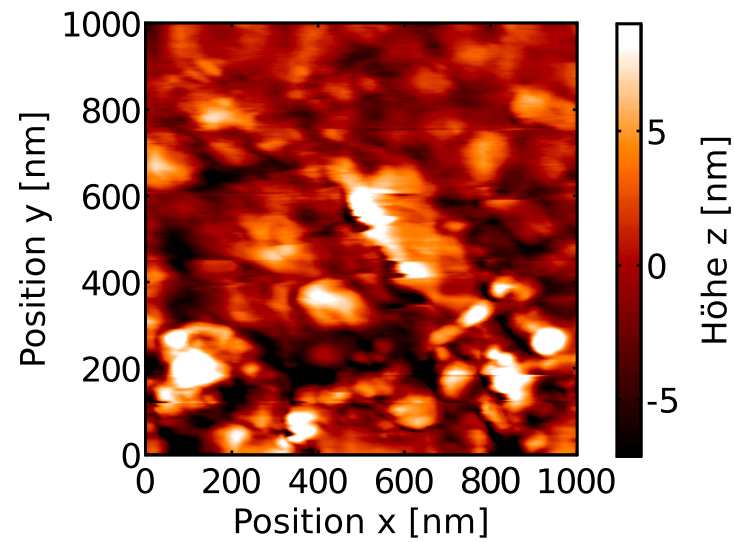

Abbildung 4.8: Bei $U=100 \mathrm{mV}$ aufgezeichnete Stromkarte (a) mit zugehöriger Topographie (b). Die isolierende Oberfläche der $\mathrm{La}_{0,85} \mathrm{Sr}_{0,15} \mathrm{MnO}_{3}$-Schicht wurde zuvor - wie in Abb. 4.7 - durch das Abfahren einiger Linien bei dauerhaft anliegender großer positiver Spannung $U=7 \mathrm{~V}$ im unteren Bildbereich umgeschaltet. Die Leitfähigkeitsänderung erstreckt sich hier aber auch in vergleichsweise weit entfernte Regionen. Dabei besteht eine deutlich erkennbare Korrelation zwischen der Ausbreitung der metallischen Region und der relativ rauen Topographie.

Abb. 4.7 zeigt eine Stromkarte (a) mit zugehöriger Topographie (b). Bei einer vorherigen Messung wurde während des Abrasterns der Oberfläche eines $\mathrm{La}_{0,85} \mathrm{Sr}_{0,15} \mathrm{MnO}_{3}$-Filmes ${ }^{\mathrm{d}}$ zweimal für jeweils einige Linien die an der Spitze anliegende Spannung auf $U=3 \mathrm{~V}$ erhöht. Die Stromkarte zeigt in diesen Bereichen jetzt eine stark erhöhte lokale Leitfähigkeit. Diese umgeschalteten Gebiete sind zwar räumlich relativ scharf begrenzt, erscheinen dabei aber nicht besonders homogen: Es ist vielmehr eine ,körnige" Struktur erkennbar, die allerdings in keiner direkten Korrelation zu der sehr glatten Topographie steht, welche von lateral deutlich ausgedehnteren Wachstumsinseln geprägt ist.

Ein extremes Gegenbeispiel hierzu besteht in der in Abb. 4.8 dargestellten Messung. Diese an der gleichen Probe aufgezeichnete Stromkarte (a) entstand nach einem ähnlichen Experiment: Es wurden im unteren Bildbereich wieder einige Linien mit erhöhter Spannung (in diesem Fall $U=7 \mathrm{~V}$ ) aufgenommen. Man erkennt jetzt jedoch einen sehr großen, sich bis zu einem Abstand von einem halben Mikrometer erstreckenden, metallischen Bereich. Es sind außerdem deutliche Korrelationen mit der Topographie (b) erkennbar. Die Leitfähigkeitsänderung hat sich also, offenbar örtlich begünstigt durch eine bestimmte Morphologie, auch weit entfernt von der eigentlichen Pulsposition ausbreiten können.

Das in Abb. 4.9 (a) gezeigte, herzförmige Muster dieser bei $500 \mathrm{mV}$ aufgenommenen Stromkarte wurde durch 41 Pulse der Stärke $U=4 \mathrm{~V}$ und Länge $\tau=100 \mathrm{~ms}$ auf der $\mathrm{La}_{0,8} \mathrm{Ca}_{0,2} \mathrm{MnO}_{3}$-Probe erzeugt. Die wieder körnig erscheinenden metallischen Regionen

\footnotetext{
${ }^{\mathrm{d}}$ Eine Dotierung von $x=0,15$ wurde in diesem Fall zwar nominell vorgegeben, der - nicht gezeigte Widerstandsverlauf ${ }^{[137]}$ zeigt aber einen eindeutigen Metall-Isolator-Übergang und lässt in Bezug auf Urushibara et. al eine Unterschätzung der Dotierung vermuten. ${ }^{[62]}$
} 
(a) Stromkarte

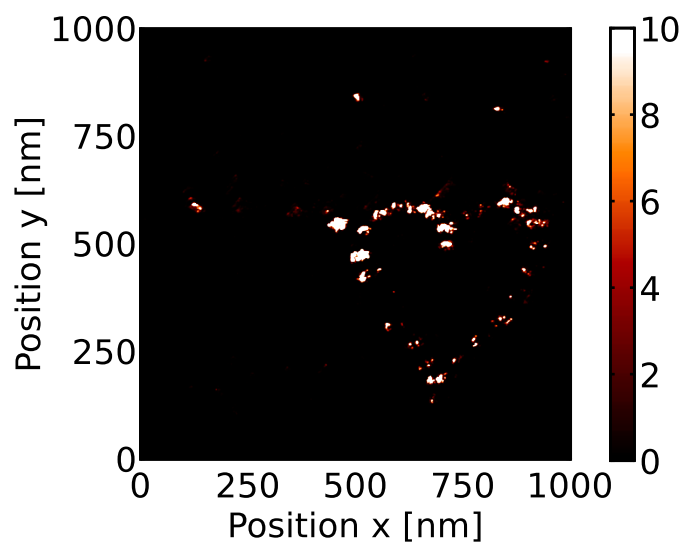

(b) Topographie

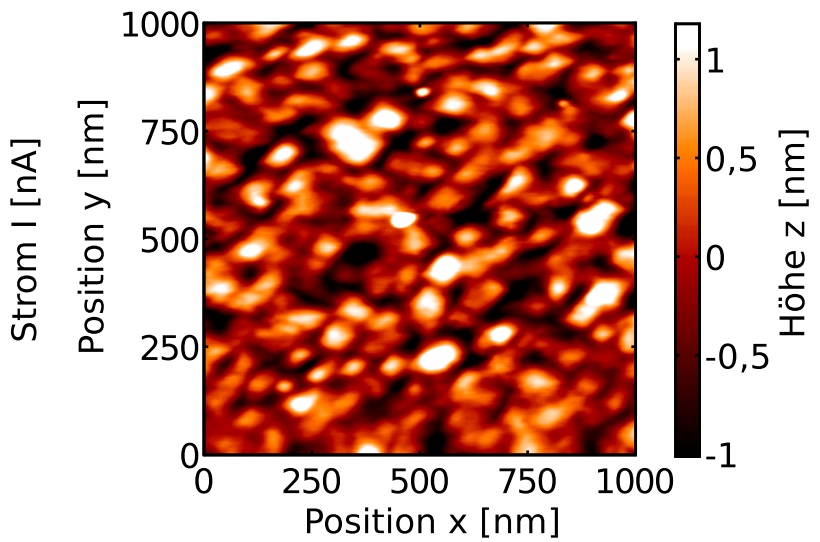

Abbildung 4.9: Stromkarte (a) mit zugehöriger Topographie (b). Das herzförmige Muster metallischer Inseln wurde durch 41 positive Spannungspulse auf der $\mathrm{La}_{0,8} \mathrm{Ca}_{0,2} \mathrm{MnO}_{3}$ Oberfläche generiert. Fernab der Pulspositionen sind ebenfalls metallisch geschaltete „Satellitenflecken" zu sehen.

spiegeln dabei nicht exakt die Pulsanordnung wieder. Es besteht außerdem keine sichtbare Korrelation mit der nebenstehend abgebildeten Topographie Abb. 4.9 (b).

Auffällig sind die mehrere hundert Nanometer von dem Herz entfernten, umgeschalteten Bereiche. Diese ,Satellitenflecken“ entstanden fernab der Pulspositionen und sind auch in einer vorher aufgenommenen Stromkarte derselben Region noch nicht beobachtet worden, also tatsächlich eine Folge der Spannungspulse.

Abb. 4.10 (a) zeigt schließlich eine weitere an der $\mathrm{La}_{0,85} \mathrm{Sr}_{0,15} \mathrm{MnO}_{3}$-Probe aufgenommene Stromkarte. Durch vorheriges Abrastern des gesamten Bildbereichs bei permanent anliegender Spannung $U>U_{\mathrm{c}}$ konnte der dargestellte Ausschnitt der $\mathrm{La}_{0,85} \mathrm{Sr}_{0,15} \mathrm{MnO}_{3}$-Probe hier vollständig in den metallischen Zustand versetzt werden, ohne dass Oberflächenmodifikationen feststellbar waren. Erst danach wurden an vier Punkten negative Spannungspulse gesetzt, welche als dunkle Regionen erkennbare, hochohmige Bereiche in einer metallischen Matrix generierten. Dieses Experiment stellt somit eine Umkehrung der zuvor beschriebenen Pulsversuche dar, welche auf einer isolierenden Oberfläche durchgeführt wurden.

Abschließend ist in Abb. 4.10 (b) die Stromkarte eines metallischen Flecks gezeigt, welcher auf der Oberfläche der $\mathrm{La}_{0,8} \mathrm{Ca}_{0,2} \mathrm{MnO}_{3}$-Probe bei einer Temperatur von $T \approx 120 \mathrm{~K}$ - also weit unterhalb der Metall-Isolator-Übergangstemperatur $T_{\mathrm{MI}}=205 \mathrm{~K}$ - geschrieben wurde. ${ }^{\mathrm{e}}$ Eventuelle Temperaturabhängigkeiten des Schalteffektes wurden in dieser Arbeit zwar nicht systematisch untersucht, es konnte jedoch kein prinzipieller Unterschied gefunden werden - ergänzend sei auf die Voruntersuchungen von Markus Esseling ${ }^{[107]}$ sowie die gegenwärtigen Arbeiten von Christin Kalkert verwiesen.

\footnotetext{
${ }^{\mathrm{e}}$ Es ist darauf hinzuweisen, dass sich im Verlauf dieser Messung moderate Oberflächenmodifikationen (nicht gezeigt) ergaben - auf diesen Effekt wird im folgenden Abschnitt 4.5.2 eingegangen.
} 
(a) Stromkarte

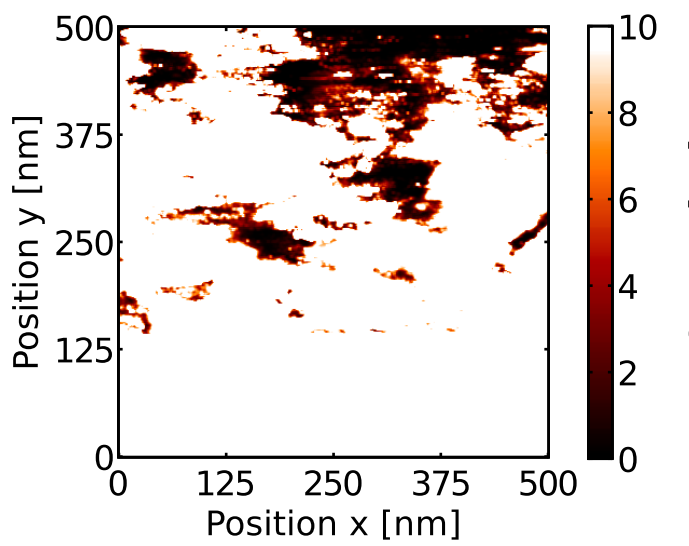

(b) Stromkarte

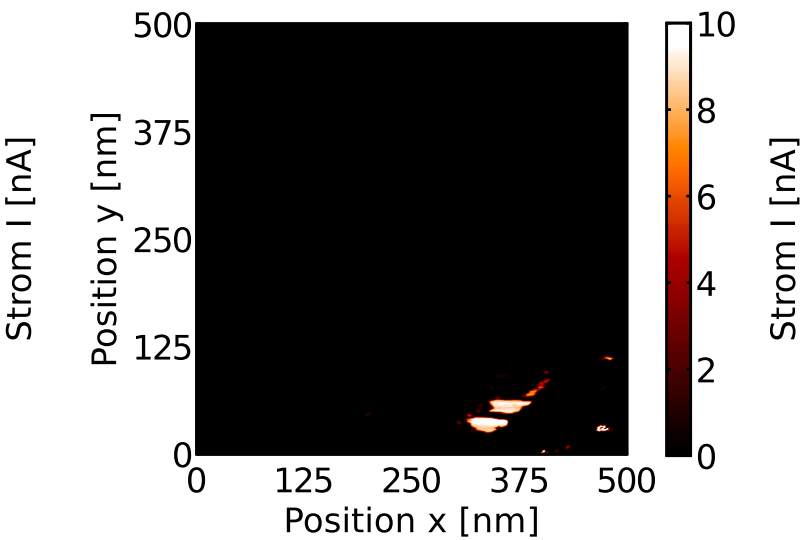

Abbildung 4.10: Stromkarten (ohne Topographie): (a) Die $\mathrm{La}_{0,85} \mathrm{Sr}_{0,15} \mathrm{MnO}_{3}$-Schicht wurde durch vorheriges, mehrmaliges Abrastern der gezeigten Region mit permanent anliegender Spannung $U>U_{\mathrm{c}}$ vollständig in den metallischen Zustand versetzt. Danach wurden durch negative Spannungspulse isolierende Bereiche in der leitenden Umgebung erzeugt. (b) Die $\mathrm{La}_{0,8} \mathrm{Ca}_{0,2} \mathrm{MnO}_{3}$-Probe wurde bei einer Temperatur $T \approx 120 \mathrm{~K}<$ $T_{\mathrm{MI}}$, untersucht. Die Oberfläche ist auch hier hochohmig und lässt sich mit positiven Spannungen in den metallischen Zustand schalten.

Die vorgestellten Stromkarten entstammen zwar lediglich einer beschränkten Probenauswahl, weitere nicht gezeigte Messungen, beispielsweise an einer $\mathrm{La}_{1 / 3} \mathrm{Pr}_{1 / 3} \mathrm{Ca}_{1 / 3} \mathrm{MnO}_{3}-$ Schicht, zeigen aber keine grundsätzlichen Unterschiede. Das Schaltverhalten der untersuchten Manganatoberflächen lässt keine starke qualitative Abhängigkeit von den die Bulkeigenschaften bestimmenden Dotierungsparametern erkennen; es kann außerdem oberund unterhalb der Übergangstemperatur beobachtet werden. Stattdessen sind große Variationen in der Form der metallischen Domänen für verschiedene Oberflächenregionen der gleichen Probe und insbesondere manchmal deutliche Einflüsse der Probenmorphologie zu verzeichnen. 


\subsubsection{Oberflächenmodifikationen}

Der Vollständigkeit halber sei an dieser Stelle eine der wesentlichen experimentellen Schwierigkeiten erwähnt, welche eine genaue Reproduzierbarkeit zusätzlich zu den inhärent statistischen Proben- und Oberflächeneigenschaften und der sich während der Messungen ändernden Spitzenqualität erschwert. Oftmals wurden nach positiven, den metallischen Zustand erzeugenden Schaltpulsen erhebliche Modifikationen der Oberfläche festgestellt. Dies waren stets deutliche Erhöhungen im Vergleich zu vorherigen Topographien, oftmals mehrere $10 \mathrm{~nm}$ hoch und somit in der Größenordnung der Schichtdicke des Manganates. Derartige Veränderungen konnten nicht durch negative Spannungspulse induziert werden und erwiesen sich zudem als irreversibel.

(a) Topographie (vorher)

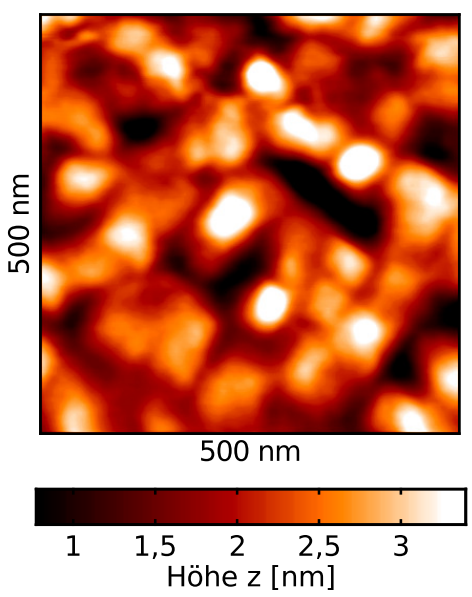

(b) Topographie (nachher)

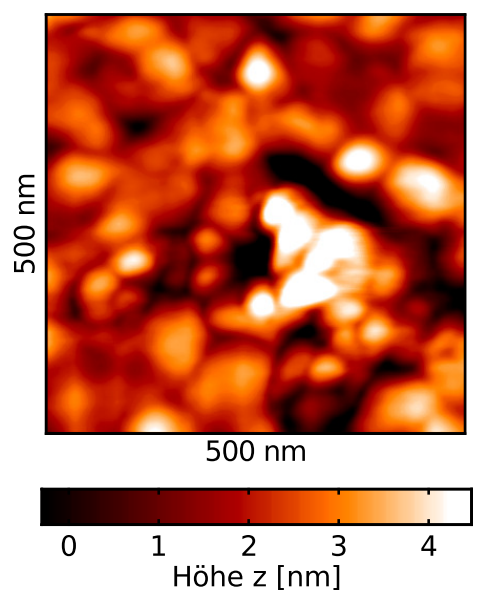

(c) Differenzbild

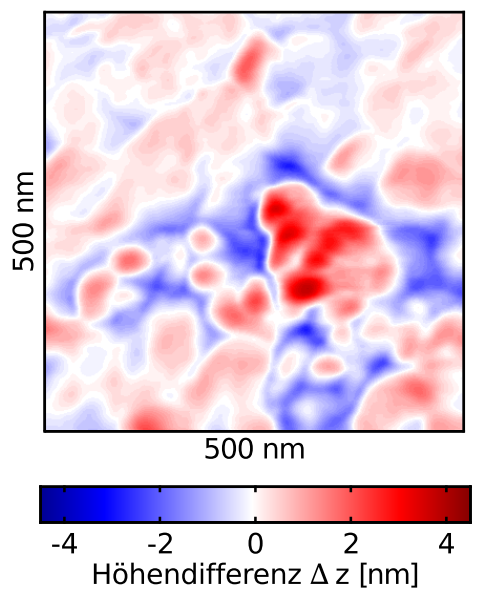

Abbildung 4.11: Oberflächenmodifikation durch einen Spannungspuls von $U=8 \mathrm{~V}$ und $\tau=1 \mathrm{~s}$. (a) und (b) zeigen die Topographie vor und nach dem Puls; im Differenzbild (c) ist eine entstandene deutliche Erhebung zu erkennen.

Als Beispiel dient an dieser Stelle Abb. 4.11, wo im topographischen Differenzbild eine Erhöhung um bis zu $5 \mathrm{~nm}$ und mit einem Durchmesser von $150 \mathrm{~nm}$ zu sehen ist, die sich durch einen $1 \mathrm{~s}$ langen Puls von $8 \mathrm{~V}$ gebildet hat. Auch nach weniger starken Pulsen stellen zum Teil noch weitaus drastischer ausgeprägte morphologische Änderungen keine Seltenheit dar.

Es sei hier bereits vorweggenommen, dass derartige unerwünschte Effekte höchstwahrscheinlich auf eine thermische Schädigung der Probe oder auch der Cantileverspitze durch hohe lokale Stromdichten zurückzuführen sind (siehe Abschnitt 5.3.4). Phänomenologisch wäre beispielsweise thermische Feldverdampfung und Ablagerung von Spitzenmaterial mit der vorliegenden Polarität vereinbar. ${ }^{[138]}$ Zur Untersuchung des Widerstandsschaltens fanden daher ausschließlich solche Messungen Verwendung, die keine oder nur unwesentliche topographische Änderungen nach Abschluss des Experiments erkennen ließen. 


\subsubsection{Domänenwachstum in Abhängigkeit von Spannung und Pulsdauer}

Das im Folgenden beschriebene Experiment spielt eine bedeutende Rolle in der späteren Diskussion, da es die räumliche Ausdehnung metallischer Domänen in Abhängigkeit von den Parametern der sie erzeugenden Spannungspulse beleuchtet. Diese Messungen werden deshalb etwas detaillierter und schon mit einigen ersten Auswertungen vorgestellt.

In Abb. 4.12 ist eine bei $100 \mathrm{mV}$ aufgenommene Stromkarte gezeigt. Vor der Messung wurde auf einer vollständig isolierenden Probenregion ein $7 \times 7$-Raster von Spannungspulsen gesetzt; dabei wurden sowohl die Pulsspannungen $U$ horizontal im Bereich $2 \ldots 8 \mathrm{~V}$ als auch deren Dauern $\tau$ vertikal im Intervall $10 \ldots 1000$ ms variiert.

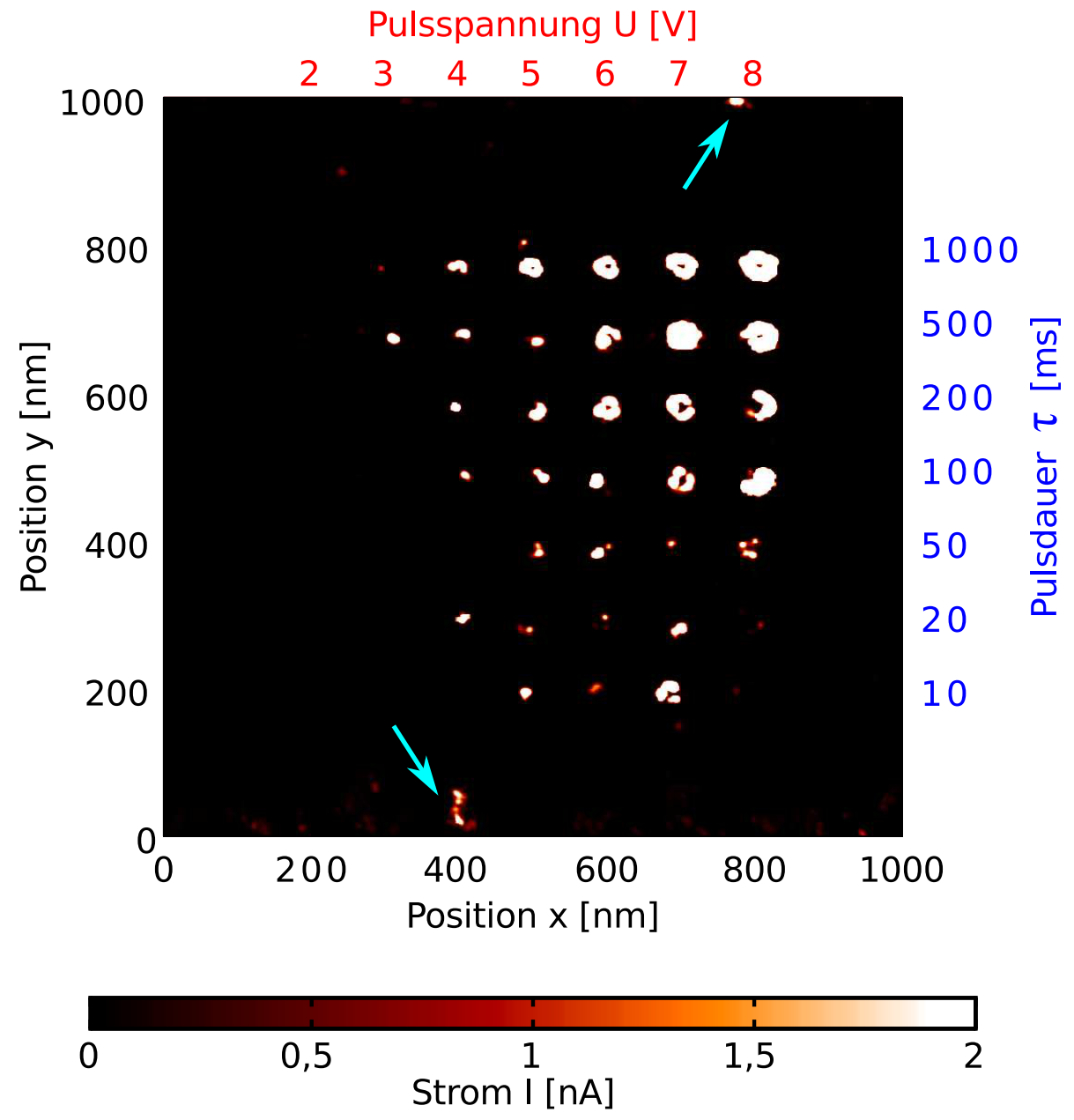

Abbildung 4.12: Bei $100 \mathrm{mV}$ aufgenommene Stromkarte nach einer rasterförmigen Anlegung von Pulsen verschiedener Spannungen $U$ und Dauern $\tau$. Man erkennt die Tendenz einer schnelleren zeitlichen Zunahme der Fleckgrößen für größere Spannungen sowie das Vorliegen einer Schwellspannung. Am oberen und unteren Rand sind „Satellitenflecken“ erkennbar. (Für eine bessere Darstellung wurde der Farbkontrast auf $2 \mathrm{nA}$ reduziert, die tatsächlichen Werte überschritten den Messbereich von $50 \mathrm{nA}$.) 
(a) Spannungsabängigkeit

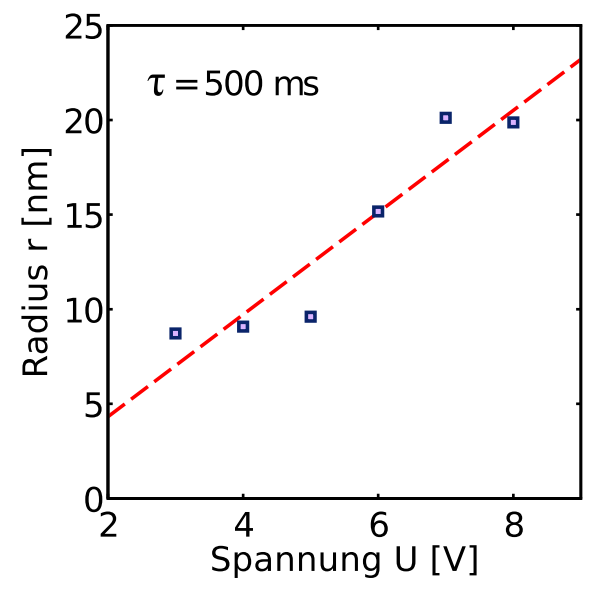

(b) Pulsdauerabhängigkeit

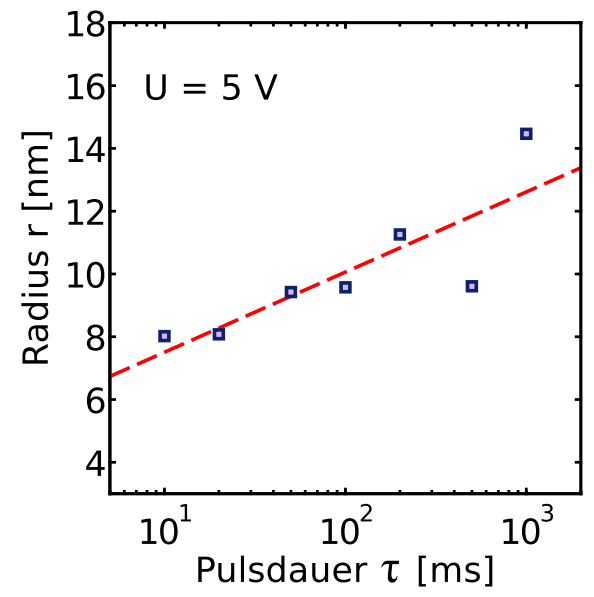

Abbildung 4.13: Abhängigkeit der Domänengröße von der Pulsamplitude (a) und Pulsdauer (b), vergleiche Abb. 4.12. Aufgetragen ist eine Spannungsreihe des numerisch bestimmten äquivalenten Radius bei fester Pulsdauer $\tau=500 \mathrm{~ms}$ sowie eine Zeitreihe für Pulse der Spannung $U=5 \mathrm{~V}$. Wie durch die ergänzten Fitfunktionen veranschaulicht, lässt sich die Spannungsabhängigkeit linear und das Zeitverhalten logarithmisch beschreiben.
An den Pulsorten erkennt man ausgedehnte metallische Inseln in der sonst isolierenden Umgebung. Pulse von nur $2 \mathrm{~V}<U_{c}$ vermochten unabhängig von ihrer Dauer keine Widerstandsänderung zu bewirken; von den verbleibenden 42 Pulsen erzeugten 39 einen metallischen „Fleck“. f Diese leitfähigen Gebiete erscheinen nahezu kreisförmig mit Durchmessern zwischen etwa $10 \mathrm{~nm}$ und $50 \mathrm{~nm}$. Klar ersichtlich ist die generelle Tendenz zu größeren Flecken sowohl für stärkere als auch längere Pulse, also einer Zunahme des Domänenradius von links unten nach rechts oben im Bild.

Die untersuchte Probenregion zeigte während dieser Messung ein zeitlich sehr stabiles Verhalten, so war das in der Nähe aufgenommene herzförmige Leitfähigkeitsbild (vgl. Abb. 4.9 (a)) über mehrere Stunden nahezu unverändert beobachtbar. Unter der Annahme, dass sich die Form und Größe der leitfähigen Domänen also auch in diesem Falle nach dem erzeugenden Pulse nicht signifikant oder nur langsam verändert hat, können die einzelnen Flecken als Stadien der zeitlichen Entwicklung während der angelegten Spannung aufgefasst werden.

Es wurde daher eine automatisierte Auswertung dieser Momentaufnahmen durch einen MATLABAlgorithmus durchgeführt, welcher die Flächen $A$ aus der Anzahl der Pixel mit Strömen oberhalb eines kritischen Wertes, in diesem Falle $I_{\text {krit. }}=5 \mathrm{nA}$, ermittelt und den äquivalenten Radius $r=\sqrt{\frac{A}{\pi}}$ einer gleich großen kreisförmigen Fläche bestimmt.

Betrachtet man nun jeweils die Größen der einzelnen Flecken in Abhängigkeit von den sie erzeugenden Spannungswerten, so bemerkt man einen im wesentlichen linearen Trend des Domänenwachstums, wie in Abb. 4.13 (a) für eine Spannungsreihe exemplarisch dargestellt. Analog wurde mit den Pulsbreiten verfahren (Abb. 4.13 (b)): Die Ausbreitung verlangsamt sich mit fortschreitender Zeit und kann gut durch ein logarithmisches Zeitgesetz beschrieben werden. Das Auftreten deutlicher Ausreißer ist in diesen Auftragungen nicht verwunderlich, da es sich ja nicht

\footnotetext{
${ }^{\mathrm{f}}$ Bei den gewählten Kontrasteinstellungen der gezeigten Stromkarte sind nicht alle 39 Flecken sichtbar.
} 
um die verschiedenen Entwicklungsstadien eines metallischen Fleckes handelt, sondern um separate Objekte.

Man erkennt in der Stromkarte außerdem, wie durch die Pfeile in Abb. 4.12 hervorgehoben, dass auch bis zu $200 \mathrm{~nm}$ abseits der eigentlichen Pulsorte metallische Regionen entstanden sind. (Diese „Satellitenflecken“ wurden in anderen Messungen sogar in weitaus größeren Entfernungen - bis zu mindestens einem Mikrometer - gefunden).

Auffällig ist ferner, dass die ausgedehnteren Flecken, welche durch intensivere Pulse erzeugt worden sind, oftmals ein isolierendes Zentrum aufweisen. Da meistens nach kürzeren

(a) Topographie vorher

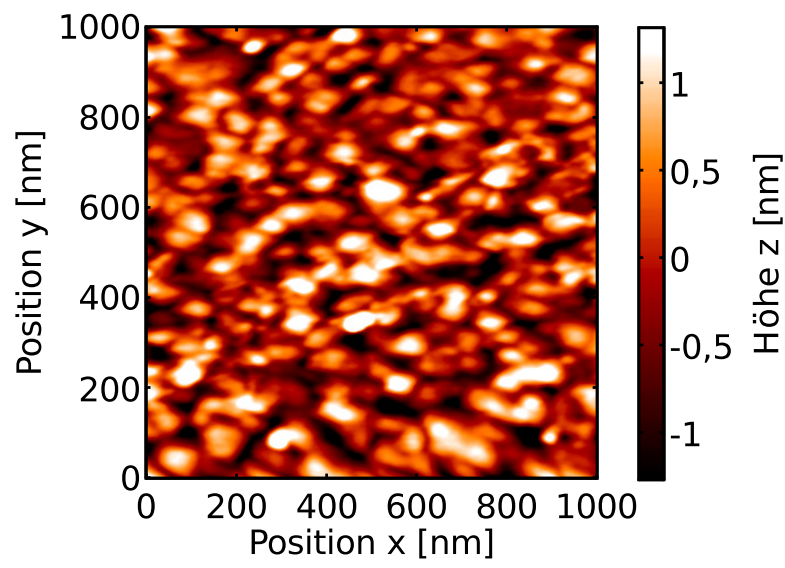

(c) Topographie nachher (vergrößert)

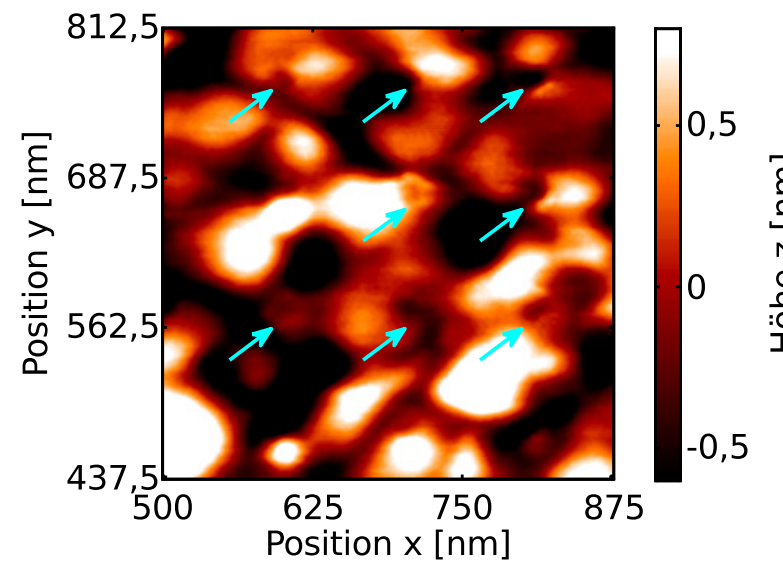

(b) Topographie nachher

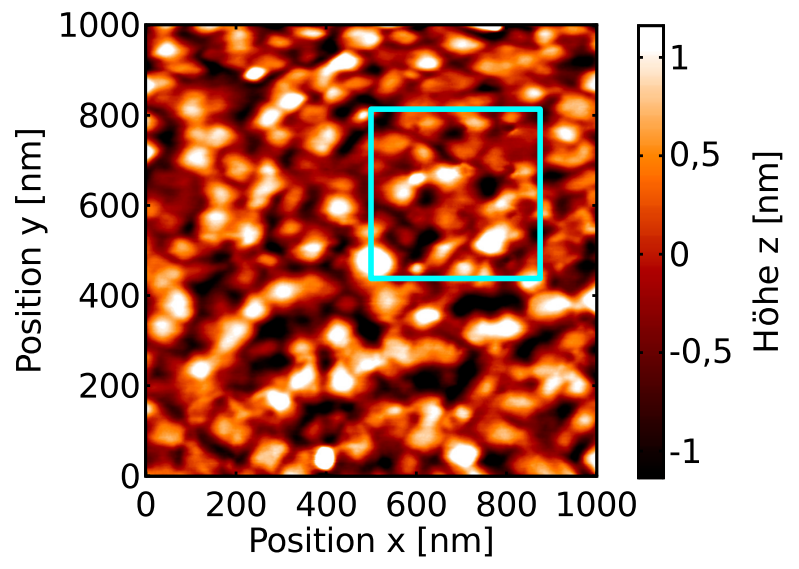

(d) Stromkarte (vergrößert)

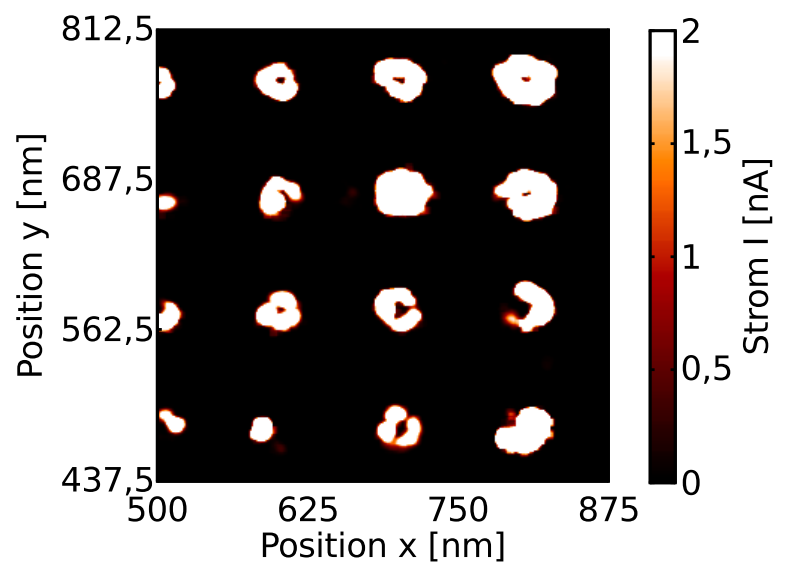

Abbildung 4.14: Entwicklung der Topograhpie während der in Abb. 4.12 gezeigten Messung. (a) und (b) zeigen die Oberfläche vor und nach dem Pulsexperiment - man beachte das deutliche Abwärtsdriften. Es sind keine starken morphologischen Modifikationen zu verzeichnen. Erst in der Vergrößerung (c) sind geringfügige Änderungen an den Positionen der stärksten Pulse erkennbar, diese wurden mit Pfeilen gekennzeichnet. Ein Vergleich mit dem entsprechenden Ausschnitt der Stromkarte (d) zeigt, dass solche Modifikationen mit der Bildung eines isolierenden Zentrums in der metallischen Domäne einhergehen. 
Pulsen der gleichen Spannung kein solcher Einbruch der Leitfähigkeit zu sehen ist, liegt die Vermutung nahe, dass dieser erst nach dem Umschalten in den metallischen Zustand entstanden ist und somit kein Relikt der isolierenden Phase darstellt.

In Abb. 4.14 ist durch einen Vergleich der Topographien vor und nach dem Pulsexperiment sowie einer vergrößerten Gegenüberstellung mit der Stromkarte ersichtlich, dass die Ausbildung der isolierenden Zentren mit einer geringfügigen topographischen Veränderung einhergeht. Bei allen anderen Flecken ist - zumindest mit dem begrenzten Aufösungsvermögen der C-AFM-Methode - keine Oberflächenmodifikation erkennbar. Wir betrachten daher, wie bereits erwähnt, diese Oberflächenveränderungen und auch den damit einhergehenden Leitfähigkeitseinbruch als ein Resultat von bei großer lokaler Stromdichte einsetzender thermischer Schädigung und nicht als Charakteristikum des Schaltverhaltens an sich.

\subsection{Zeitabhängige Messungen}

In diesem Abschnitt werden die Ergebnisse tatsächlich zeitaufgelöster Experimente vorgestellt. Dabei wird zunächst die Beobachtung der langsamen Rückbildung einer einzelnen metallischen Domäne besprochen. Anschließend werden Experimente mit Pulsserien behandelt, während denen der Cantilever an verschiedenen Rasterpunkten ortsfest auf der Oberfläche verweilt.

\subsubsection{Rückbildung einer metallischen Domäne}

Während im vorigen Abschnitt nur das Resultat verschiedener Pulslängen betrachtet wurde - und dabei das Vorliegen eines langzeitstabilen Zustandes nach dem Pulsende vorausgesetzt werden musste (vergleiche Abschnitt 4.5.3) - wenden wir uns nun tatsächlich zeitaufgelösten Messungen zu.

Im Gegensatz zu dem oben dargestellten, selbst über Stunden stabilen Domänenbild können auch sich zurückbildende metallische Regionen beobachtet werden. Meistens finden diese Prozesse vergleichsweise rasch statt: Setzt man einen Schaltpuls und nimmt unmittelbar darauf eine Stromkarte des betroffenen Gebietes auf, ist keine Änderung der lokalen Leitfähigkeit mehr festzustellen. Nimmt man hingegen eine Stromkarte bei einer Spannung über dem Schwellwert auf, so fließt trotzdem ein für den LRS typischer, sehr großer Strom - die Widerstandsänderung findet also statt, ist jedoch nicht remanent und die Domänenausbreitung nicht abbildbar.

Gelegentlich ist es hingegen möglich, mit rasch aufeinanderfolgenden Messungen das graduelle Schrumpfen eines metallisch geschalteten Bereichs direkt zu verfolgen. An der $\mathrm{La}_{0,8} \mathrm{Ca}_{0,2} \mathrm{MnO}_{3}$-Probe konnte die sehr langsame Rückbildung einer einzelnen metallischen Domäne, deren Zeitskala eine Vielzahl aufeinander folgender, ortsaufgelöster Messungen gestattete, beobachtet werden. Abb. 4.15 zeigt anhand von ausgewählten Momentaufnahmen, wie zunächst mehrere relativ große, leitfähige Gebiete entstehen, von denen jedoch nach wenigen Minuten nur noch eines übrig ist. Die Rückbildung dieser Domäne erstreckt sich über einen Zeitraum von etwa 45 Minuten.

Es wurde wieder eine Quantifizierung des Domänenradius nach dem zuvor beschriebenen Verfahren durchgeführt. Dabei wurde diesmal ein für jede Stromkarte dynamisch angepasster Schwellwert von $10 \%$ des maximal gemessenen Wertes gewählt, wodurch auch die späteren 


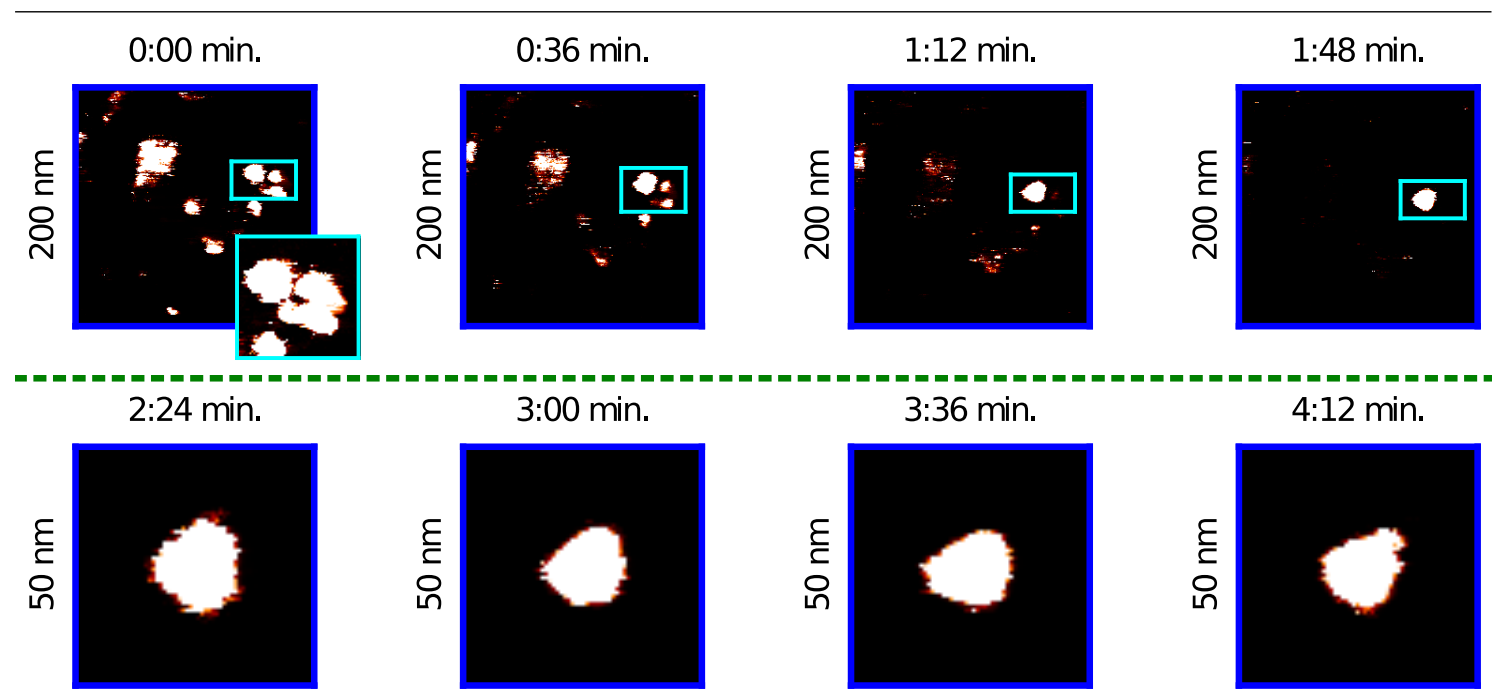

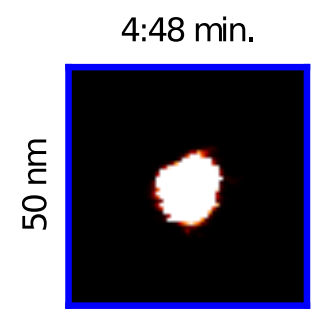

12:00 min.
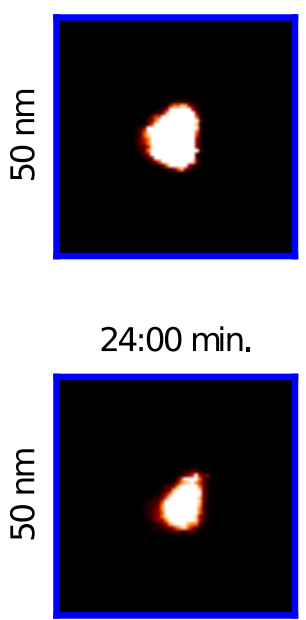

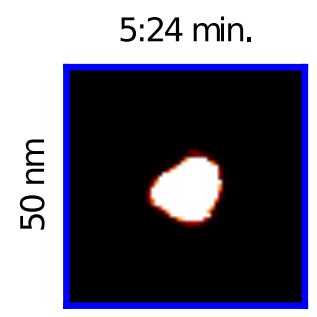

15:00 min.
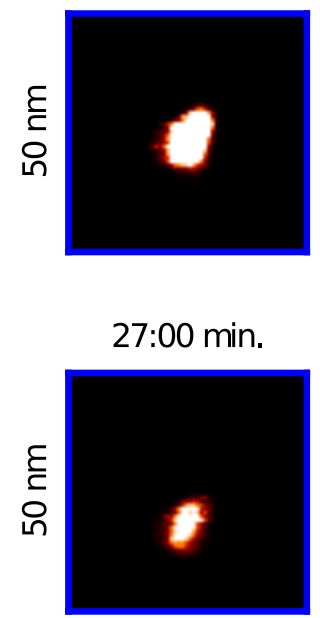

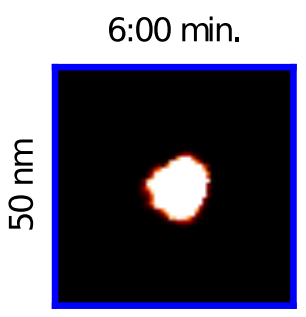

18:00 min.
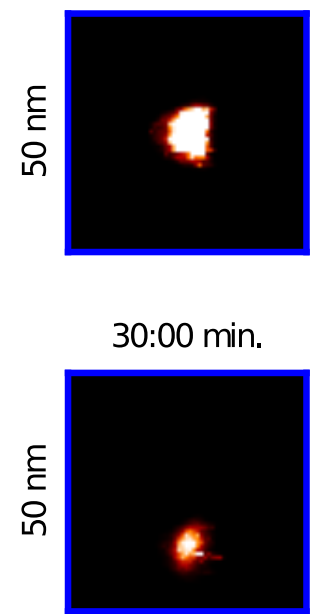

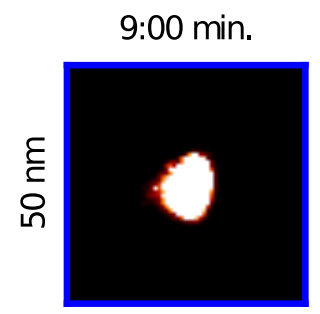

21:00 min.

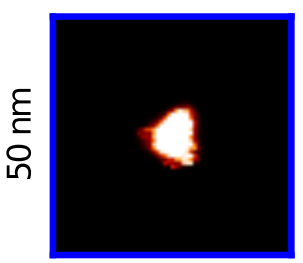

33:00 min.

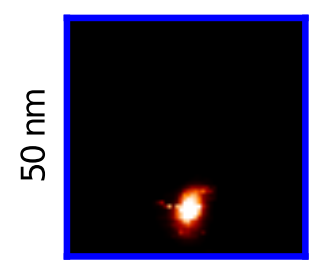

Abbildung 4.15: Ausgewählte Stromkarten der Rückbildung einer metallischen Domäne. Auf eine Farbskala wurde verzichtet; der Kontrastbereich erstreckt sich von 0 nA (schwarz) bis $1 \mathrm{nA}$ (weiß). Das zuvor vollständig isolierende Gebiet weist nach dem Schaltpuls zunächst mehrere ausgedehnte metallische Regionen auf, zur Ermittelung des äquivalenten Radius (Abb. 4.16) des aus drei benachbarten Flecken bestehenden Clusters wurde anfangs nur das hellblau umrahmte Gebiet analysiert. Die Vergrößerung der ersten Stromkarte mit empfindlicherer Kontrasteinstellung illustriert, dass es sich tatsächlich um ein zusammenhängendes Objekt handelt. Ab der fünften Messung haben sich bis auf einen Fleck alle metallischen Regionen zurückgebildet. Von hier an sind die Stromkarten vergrößert dargestellt. 
Aufnahmen, bei welchen nicht nur die Ausdehnung der Domäne, sondern auch die maximale Größe der Stromwerte verringert ist, in die Analyse mit einbezogen werden konnten. Für die ersten vier Stromkarten wurden die numerische Analyse auf einen geeigneten Bildausschnitt beschränkt, der nur die am langsamsten schrumpfende Domäne umfasst. Für Zeiten ab etwa zwei Minuten liegt nur noch eine einzige metallische Domäne vor. Es zeigt sich, wie in Abb. 4.16 erkennbar, ein mit einer logarithmischen zeitlichen Abnahme zumindest konsistentes Verhalten - ebenso gut wäre jedoch eine Approximation durch zwei lineare Regime denkbar.

Die zeitliche Rückbildung metallischer Domänen kann also mit sehr unterschiedlichen Geschwindigkeiten erfolgen. Im Vergleich mit dem eher auf der Millisekundenskala angesiedelten, im vorigen Abschnitt studierten Wachstum während der Pulse vollzieht sie sich oftmals sehr langsam - bis hin zu möglicherweise stabilen Konfigurationen.

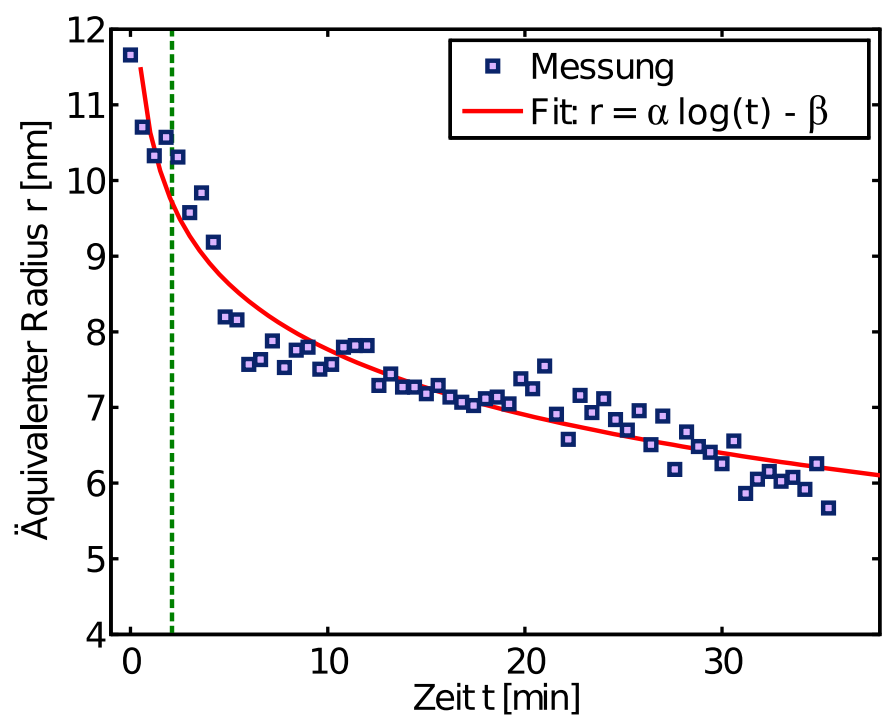

Abbildung 4.16: Zeitabhängigkeit eines numerisch bestimmten, äquivalenten Domänenradius zu Abb. 4.15 (vergleiche auch Abb. 4.12). Alle Stromwerte oberhalb von 5\% des Maximalwertes einer Karte wurden der metallischen Domäne zugeordnet. Die eingetragene Fitfunktion verdeutlicht, dass die Rückbildung einem logarithmischen Trend folgt. Ab der grünen Linie liegt nur noch eine metallische Domäne vor.

\subsubsection{Leitfähigkeitsanstiegs- und -abklingverhalten in Pulsserienversuchen}

Dieser Abschnitt ist Experimenten mit komplexen Pulsfolgen gewidmet. Im Vordergrund steht hier eine phänomenologische Erfassung des Wechselspiels zwischen der selbstständigen Rückbildung der metallischen Bereiche zwischen den Einzelpulsen einerseits und einer möglichen Akkumulation zu einer größeren Widerstandsänderung andererseits. Die hier gezeigten Messungen wurden an der $\mathrm{La}_{0,8} \mathrm{Ca}_{0,2} \mathrm{MnO}_{3}$-Probe gewonnen; dabei wurden auf Gebieten von jeweils $1000 \times 1000 \mathrm{~nm}^{2}$ an vielen, rasterartig angeordneten Positionen Folgen von Spannungspulsen angelegt. 
Abb. 4.17 (a) demonstriert die Vorgehensweise: Es wurden abwechselnd Spannungspulse der Stärke $U_{\mathrm{a}}$ und Dauer $\tau_{\mathrm{a}}$ gesetzt und Pausen der Länge $\tau_{\mathrm{b}}$ eingehalten, in welchen bei kleinerer Spannung $U_{\mathrm{b}}$ der Strom gemessen wurde. Diese Strommessung erfolgte stets in den letzten $320 \mu$ s von $\tau_{\mathrm{b}}$, es wurde also die Leitfähigkeit direkt vor dem nachfolgenden Puls gemessen. ${ }^{\mathrm{g}}$ Die komplexen Pulsprogramme bestanden jeweils aus mehreren solcher Pakete. Nach jeder Folge von positiven Pulsen wurde dabei eine kürzere Serie bei negativer Spannung aufgenommen, um den Ausgangszustand wiederherzustellen.

(a) Pulsfolge (schematisch)

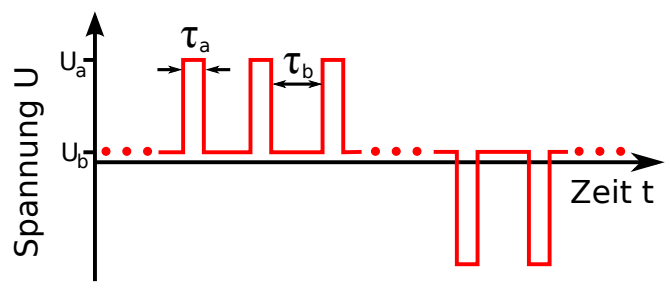

(b) Exemplarische Stromverläufe

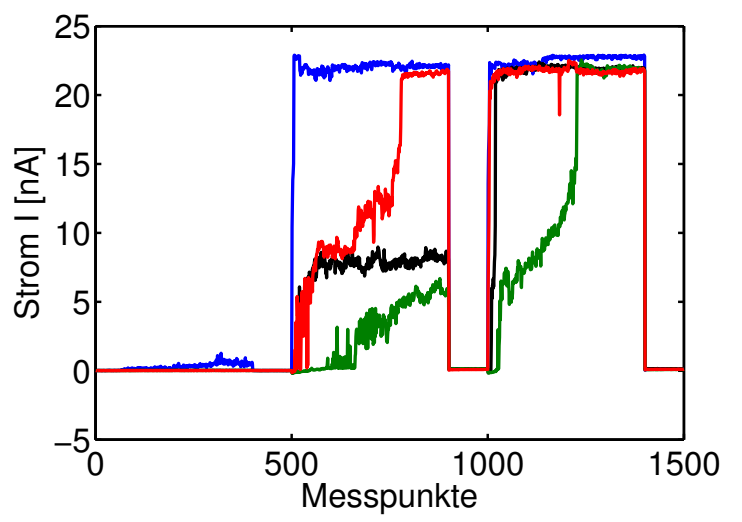

Abbildung 4.17: Pulsserienexperimente. Die schematische Pulsfolge (a) erklärt die Nomenklatur der Parameter. Ein Experiment besteht aus mehreren Pulsserien verschiedener Längen und Parameter. Exemplarische Stromverläufe (b) aus der in Abb. 4.18 (b) gezeigten Messung illustrieren die großen Variationen zwischen den Einzelkurven.

Wie in Abb. 4.17 (b) zu sehen ist, ist die lokale Reaktion der Probe sehr starken Schwankungen unterworfen. Während ein Großteil der Messungen überhaupt kein erfolgreiches Widerstandsschalten aufweist, zeigen einige einen quasi instantanen (blaue Kurve), andere eher langsame (grün) oder auch mehrstufige Anstiege der Leitfähigkeit (rot). Das Zurückschalten unter negativer Spannung ist in den meisten Fällen deutlich rascher. Dennoch scheint in vielen Fällen auch nach einer eingeschobenen negativen Pulsserie die nächste positive Folge nicht wieder beim Ausgangszustand zu beginnen, sondern erreicht schon nach wenigen Pulsen wieder das Endniveau der vorherigen, positiven Spannungsfolge (rote Kurve).

Insbesondere in den gemittelten Stromverläufen (siehe Abb. 4.18) wird deutlich, dass eine starke Abhängigkeit von der Wartezeit $\tau_{\mathrm{b}}$ zwischen den Pulsen besteht. So gelingt es, wie in (a) dargestellt, hier bei $\tau_{\mathrm{a}}=0,5 \mathrm{~ms}$ langen $5 \mathrm{~V}$-Pulsen mit Wartezeiten von $\tau_{\mathrm{b}}=0,5 \mathrm{~ms}$ und $\tau_{\mathrm{b}}=1 \mathrm{~ms}$ die Probe in einen metallischeren Zustand zu schalten und die lokale Leitfähigkeit während der Pulsfolge sukzessive zu erhöhen. Kürzere Verzögerungen bewirken dabei einen deutlich steileren Anstieg, was auf eine rückläufige Entwicklung der metallischen Bereiche zwischen den Pulsen hinweist. Das Zurückschalten ist ferner mit einer viel geringeren Anzahl von Pulsen möglich - bereits nach dem ersten Gegenpuls ist oft keine

\footnotetext{
${ }^{g}$ Dementsprechend beträgt die eigentliche Abklingzeit der Leitfähigkeit bis zu Beginn der Messung nicht $\tau_{\mathrm{b}}$, sondern $\tau_{\mathrm{b}}-320 \mu \mathrm{s}$; eine eventuelle weitere Abnahme wird über das Messintervall gemittelt.
} 
(a) Pulsfolgen mit mittlerer und kurzer Wartezeit $\tau_{b}$

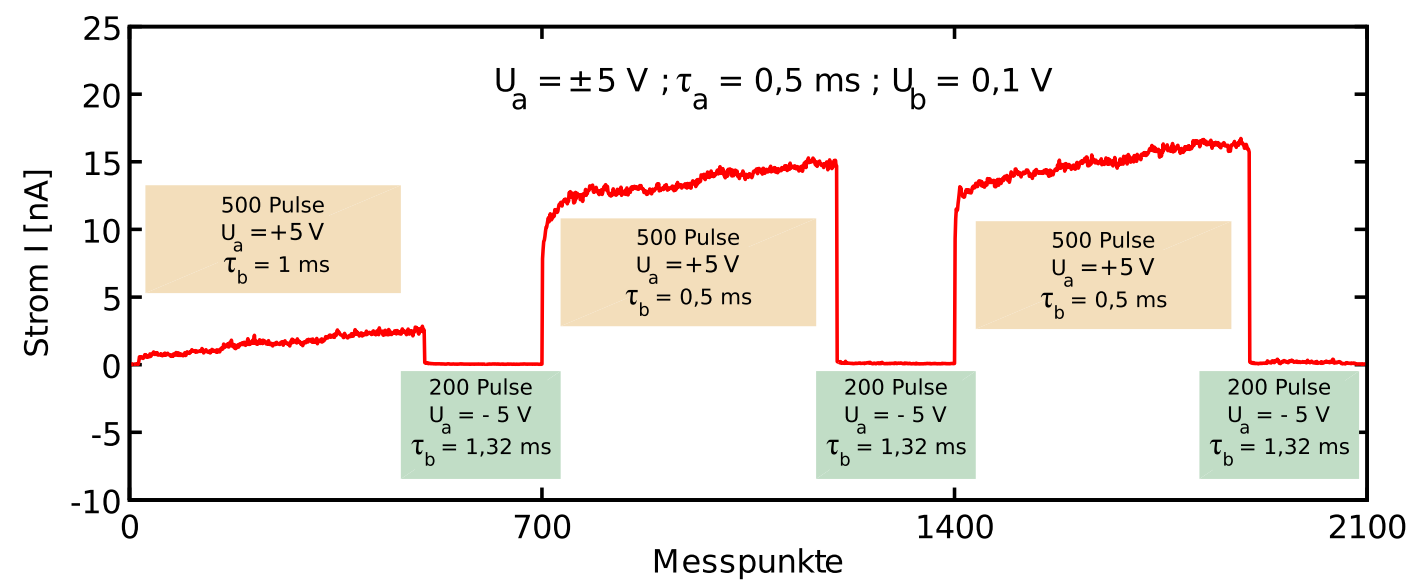

(b) Pulsfolgen mit langer und kurzer Wartezeit $\tau_{b}$

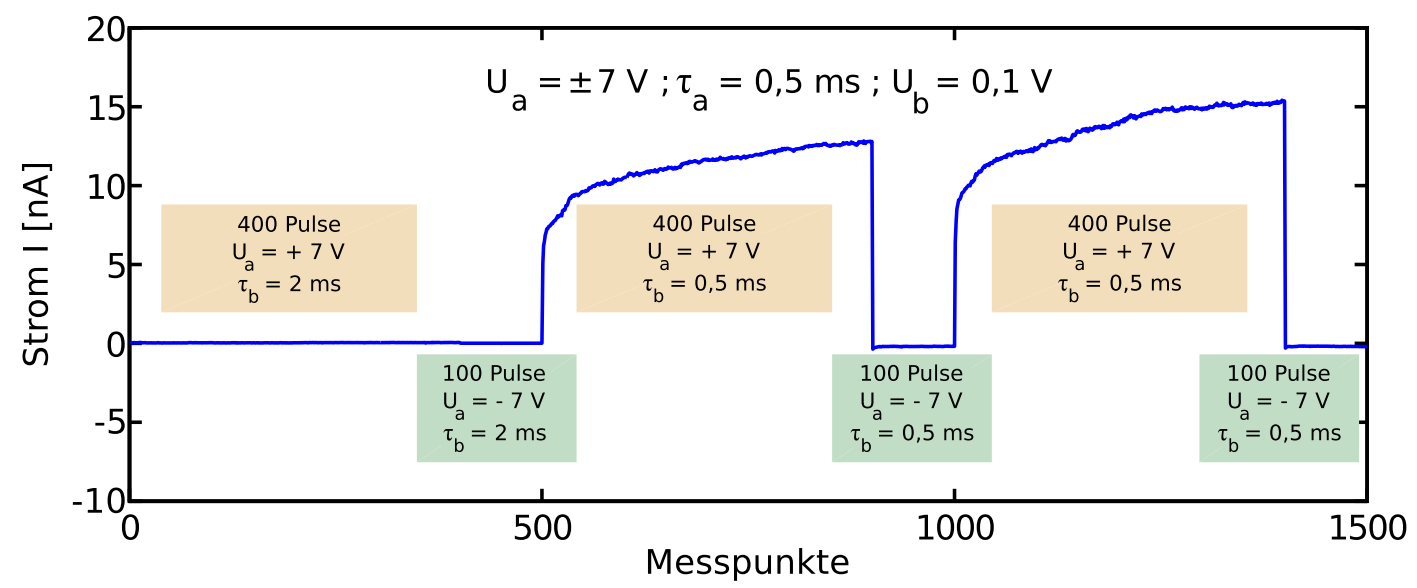

Abbildung 4.18: Stromentwicklung im Verlauf von Pulsfolgen. Man erkennt, dass längere Wartezeiten zwischen den Pulsen in einer verringerten Leitfähigkeit resultieren. In (a) ist das gemittelte Ergebnis aus 57 erfolgreichen Einzelkurven eines Rasters von $11 \times 11$ Messungen gezeigt, bei denen der maximale Stromwert eine Grenze von $2 \mathrm{nA}$ überstieg. Für (b) wurden 94 von $21 \times 21$ Kurven ausgewählt. Die beiden Messungen entstanden nicht am gleichen Probenort.

signifikant erhöhte Leitfähigkeit mehr feststellbar. Wie bereits anhand von Abb. 4.17 (b) gezeigt, scheinen gewisse Remanenzen jedoch auch eine längere Folge von Gegenpulsen zu überdauern, was sich hier in der höheren erzielten Leitfähigkeit einer zweiten positiven Pulsserie widerspiegelt.

Abb. 4.18 (b) zeigt ein grundsätzlich ähnliches Verhalten, hier wurde jedoch in der ersten Pulsfolge eine noch längere Wartezeit, $\tau_{\mathrm{b}}=2 \mathrm{~ms}$, gewählt, bei welcher trotz einer etwas höheren Pulsamplitude von $U_{\mathrm{a}}=7 \mathrm{~V}$ eine Veränderung der Leitfähigkeit praktisch gänzlich ausbleibt. Die in Folge der anliegenden Spannung wachsenden metallischen Domänen, deren Vorhandensein die nachfolgenden Pulsserien mit kürzerem $\tau_{\mathrm{b}}$ ja belegen, bilden sich bis zum Beginn der nächsten Strommessung wieder vollständig zurück. 


\subsection{Ergänzungen: Probenspezifische Untersuchungen}

Die bisherigen Ergebnisse zeigten das Widerstandsschalten als eine universale Eigenschaft der Oberflächen verschiedener Manganatproben. Variationen durch eine veränderte Dotierung und auch die Temperaturabhängigkeit wurden hingegen nicht systematisch untersucht. Schon für eine einzelne Probe sind quantitative Aussagen aufgrund der Kombination aus der für Manganate spezifischen, räumlichen Inhomogenität und der sich ändernden mechanischen und elektrischen Kontaktbedingungen - insbesondere der raschen Alterung der Cantileverbeschichtung und den Oberflächenmodifikationen im Zuge von Spannungspulsen - extrem erschwert. Derartige systematische Untersuchungen in Abhängigkeit von sonst fein durchstimmbaren Präparationsparametern konnten daher nicht durchgeführt werden. Stattdessen können aber durch drastischere Eingriffe wie die nachträgliche Modifikation der Oberfläche gezielt auch strukturelle Änderungen erzwungen und ihr Einfluss auf das Schaltverhalten studiert werden.

In diesem Abschnitt werden zunächst Resultate einer LCMO-Probe dargelegt, deren Oberflächenbeschaffenheit durch eine Terminierung mit zwei Monolagen undotiertem Lanthanmanganat verändert wurde. Es folgen Ergebnisse für eine LSMO-Probe, die ein sonst nicht beobachtetes, anscheinend über diskrete Werte verlaufendes Leitfähigkeitsverhalten, offenbaren. Ob es sich hierbei jedoch unbedingt um eine für dieses spezielle Probensystem charakteristische Eigenschaft handelt, ist unklar, da bisher nur an einer der untersuchten LSMO-Schichten diese Eigenschaft bemerkt wurde.

\subsubsection{Untersuchung einer LMO-terminierten Schicht}

Ein $\mathrm{La}_{0,7} \mathrm{Ca}_{0,3} \mathrm{MnO}_{3}$-Film wurde abschließend mit einer etwa zwei Monolagen starken Decklage undotierten Lanthanmanganates beschichtet. Die Dicke der LMO-Terminierung wurde dabei allein durch die deponierte Stoffmenge vorgegeben, es wurde auf die Erfahrungswerte der vorangegangenen Arbeiten von Kai Gehrke et al. zurückgegriffen. ${ }^{[77,78]}$ Eine tatsächliche Analyse der Schichtstruktur - etwa durch TEM-Profilaufnahmen - wurde hier jedoch nicht wiederholt.

In Abb. 4.20 (b) ist das gemittelte Widerstandsverhalten eines $41 \times 41$-Rasters von $I$ - $U$-Kennlinien dargestellt; Abb. 4.19 zeigt eine unmittelbar darauf bei $U=1 \mathrm{~V}$ aufgenommene Stromkarte. Die gleichzeitig aufgezeichnete Topographie dieser Probenregion, welche zuvor keinen Spannungsbeträgen von mehr als $|U|=1 \mathrm{~V}$ ausgesetzt worden war, ist in Abb. 4.20 (a) gegeben.

Die Stromkarte und die Kennlinie lassen gleichsam eine im Vergleich zu den Ausgangszuständen der übrigen im Rahmen dieser Arbeit untersuchten Manganatproben drastisch höhere Leitfähigkeit erkennen. Die Probe erweist sich in der gezeigten Region nahezu flächendeckend als metallisch. An anderen, nicht LMO-terminierten Proben wurde in Hunderten von Messungen niemals ein derartiges Verhalten beobachtet - ohne Spannungspulse konnten sonst lediglich vereinzelt kleine metallische Flecken in einer hochisolierenden Matrix festgestellt werden. Andere Regionen der LMO-terminierten Probe zeigten sich zwar auch isolierend; diese Variationen im Leitfähigkeitsverhalten sind aber möglicherweise einer unvollständigen Benetzung mit LMO geschuldet. 


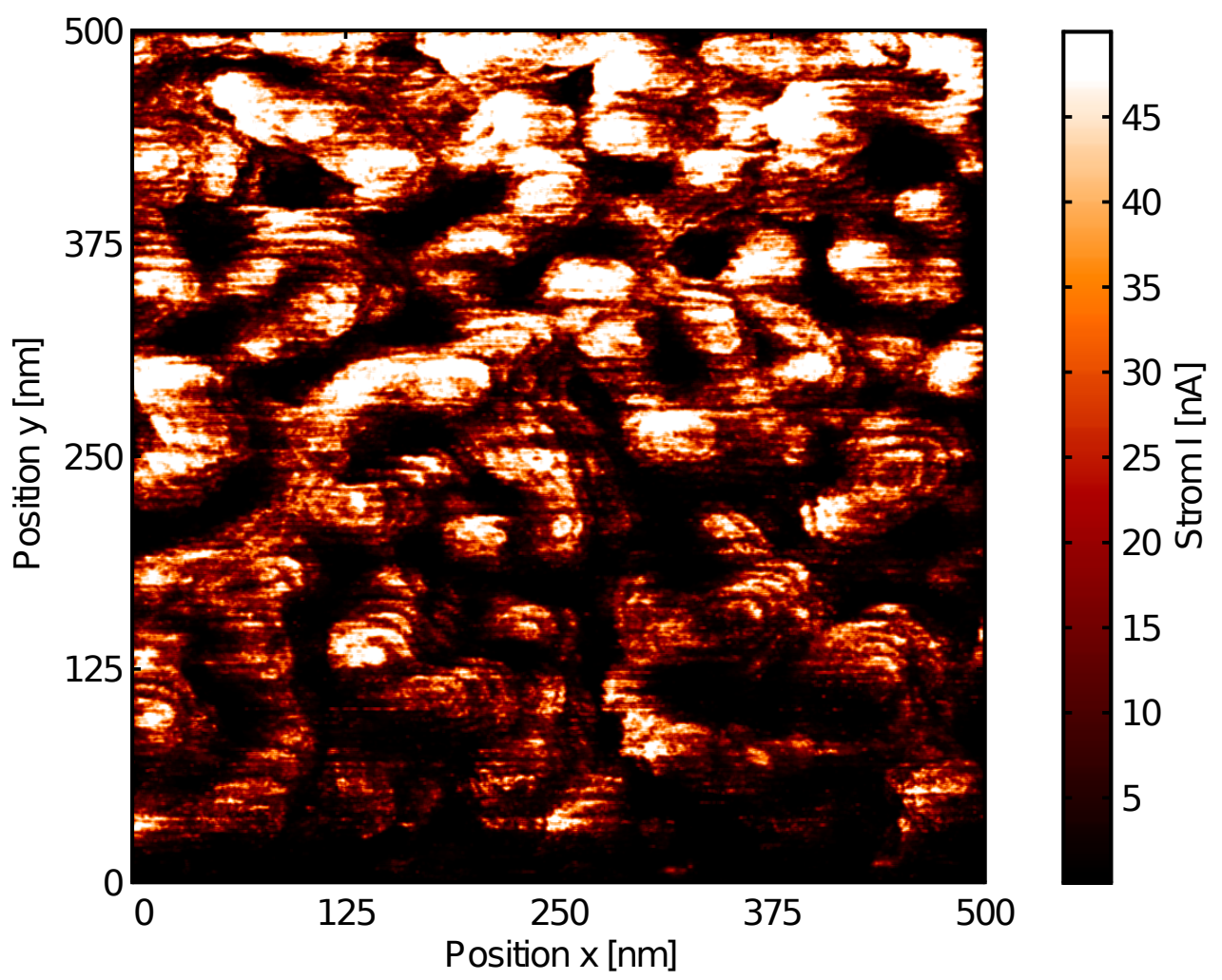

Abbildung 4.19: Stromkarte einer LMO-terminierten LCMO-Schicht, aufgenommen bei $U=1 \mathrm{~V}$. Die Leitfähigkeit ist im Vergleich zu anderen Proben stark erhöht; man erkennt außerdem eine sehr starke Korrelation mit der Topographie (vergleiche Abb. 4.20 (a)).

Die Probe besitzt demnach einen metallischen Ausgangszustand. ${ }^{\mathrm{h}}$ Es wäre zwar auch eine stark erniedrigte Schwellspannung $U_{\mathrm{c}}$ denkbar, dagegen spricht aber die auch für negative Spannungen gemessene erhöhte Leitfähigkeit. Im Verlauf der einzelnen in Abb. 4.20 (b) eingeflossenen Kennlinien ereignen sich keine abrupten Sprünge.

Auffällig ist die starke Korrelation zwischen der lokalen Leitfähigkeit und der Topographie, welche sich nicht nur auf die im Rahmen der Abbildungsfähigkeit der Spitze erkennbaren Hügel und Senken mit typischen Durchmessern von einigen zehn Nanometern beschränkt. Es hat vielmehr den Anschein, als würden sich in der Stromkarte sogar atomare Terrassen widerspiegeln - man betrachte hier zum Vergleich Abb. 4.3. Dass man derartige Eigenschaften im Stromkontrast, nicht jedoch topographisch, erfassen kann, spricht für eine recht homogene, metallische Leitfähigkeit: Der große Spitzenradius verhindert zwar eine Abbildung atomarer Terrassen, der Leitwert des Kontaktes zwischen Spitze und Probe

\footnotetext{
${ }^{\mathrm{h}}$ In anderen, hier nicht gezeigten Messungen wurden auch an dieser Probe Schalteffekte mit der gleichen Polarität wie an den übrigen Systemen beobachtet. Es handelt sich also tatsächlich vor allem um einen anderen Ausgangszustand, der aber im Nachhinein durch Spannungspulse durchaus noch modifiziert werden kann.
} 
(a) Topographie

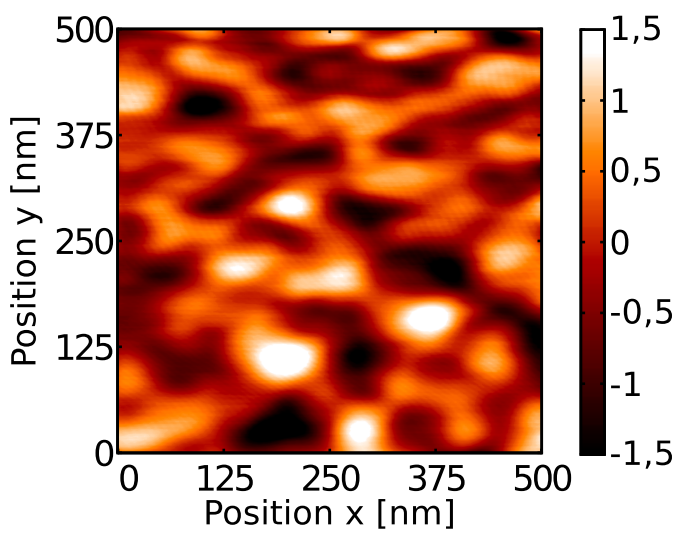

(b) I-V-Kennlinie

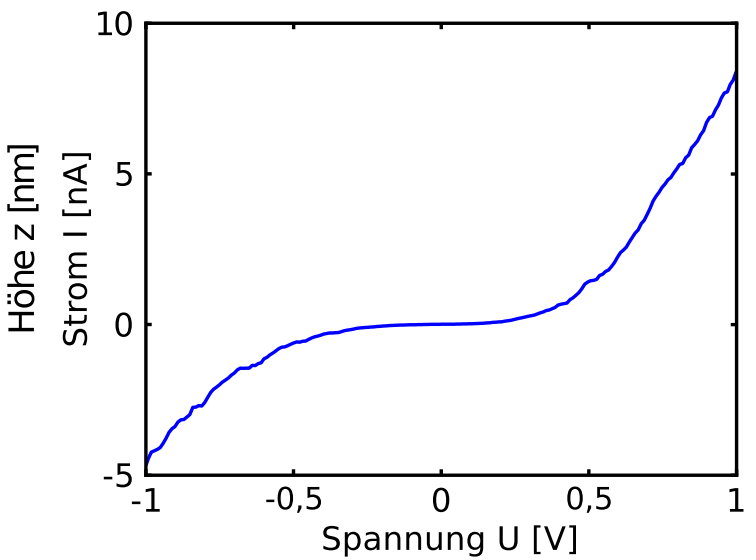

Abbildung 4.20: Topographie und $I-U$-Charakteristik des LMO-terminierten Films. Die Höhenkarte (a) wurde simultan mit der in Abb. 4.19 gezeigten Stromkarte erfasst; die $I-U$-Kennlinie (b) enstand durch Mittelung eines zuvor an der gleichen Stelle aufgenommenen Rasters von $41 \times 41$ Einzelspektren.

wird im Falle einer konstanten oder schwach variierenden Leitfähigkeit jedoch durch den Kontaktquerschnitt bestimmt. Die Auflagefläche verändert sich in der Nähe von Stufen stark, ist jedoch auf einer Terrasse ansonsten idealerweise konstant.

\subsubsection{Diskrete Leitfähigkeitswerte einer LSMO-Oberfläche}

Abschließend wird nun eine Messung besprochen, welche ausgedehnte Regionen mit homogenen, anscheinend diskreten Leitfähigkeitswerten aufweist. Dieser Beobachtung ist im Rahmen der vorliegenden Arbeit nicht systematisch durch weitere Experimente nachgegangen worden; insbesondere die Frage, ob ein derartiges Verhalten einen Sonderfall für die untersuchte Probe oder ihre Oberflächenbeschaffenheit darstellt, oder prinzipiell auch an anderen Systemen aufzufinden ist, wurde nicht geklärt. Dennoch sind möglicherweise auch schon diese ersten, qualitativen Feststellungen für eine Aufklärung des Schaltmechanismus hilfreich und müssen hier Erwähnung finden.

Abb. 4.21 zeigt eine bei $U=0,1 \mathrm{~V}$ aufgenommene Stromkarte, die zugehörige Topographie ist in Abb. 4.22 dargestellt. Der Aufnahme dieser Bilder ging eine vergleichsweise umfangreiche Reihe von Manipulationsschritten durch verschiedene Spannungen voraus: Es wurden zunächst zwei aufeinanderfolgende Scans mit einer permanent anliegenden positiven Spannung von $U=6 \mathrm{~V}$ durchgeführt. Die untersuchte Probenregion befand sich daraufhin vollständig im metallischen Zustand.

Anschließend wurde etwa an den vier durch Pfeile gekennzeichneten Punkten jeweils ein $\tau=100 \mathrm{~ms}$ langer $U=-7 \mathrm{~V}$ starker Spannungspuls gesetzt. Während der untere linke Puls keinen Übergang zurück zum hochohmigen Zustand induzieren konnte, entstand um die rechte untere Pulsposition eine ausgedehnte, im Vergleich zur Umgebung isolierende 


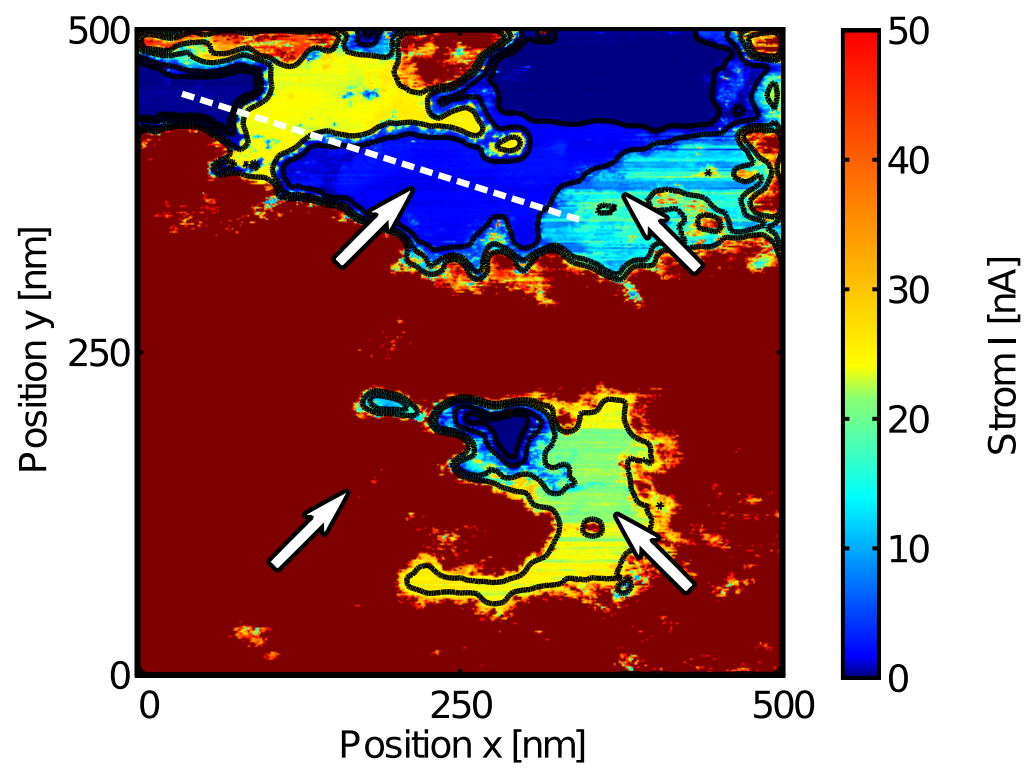

Abbildung 4.21: Stromkarte einer LSMO-Probe für $U=1 \mathrm{~V}$. Die abweichende Farbskala wurde zur Hervorhebung der Regionen homogener Leitfähigkeit gewählt. Die Pfeile markieren die Positionen der negativen Spannungspulse. Die gestrichelte Linie markiert den für die in Abb. 4.24 gezeigten Profile gewählten Pfad.

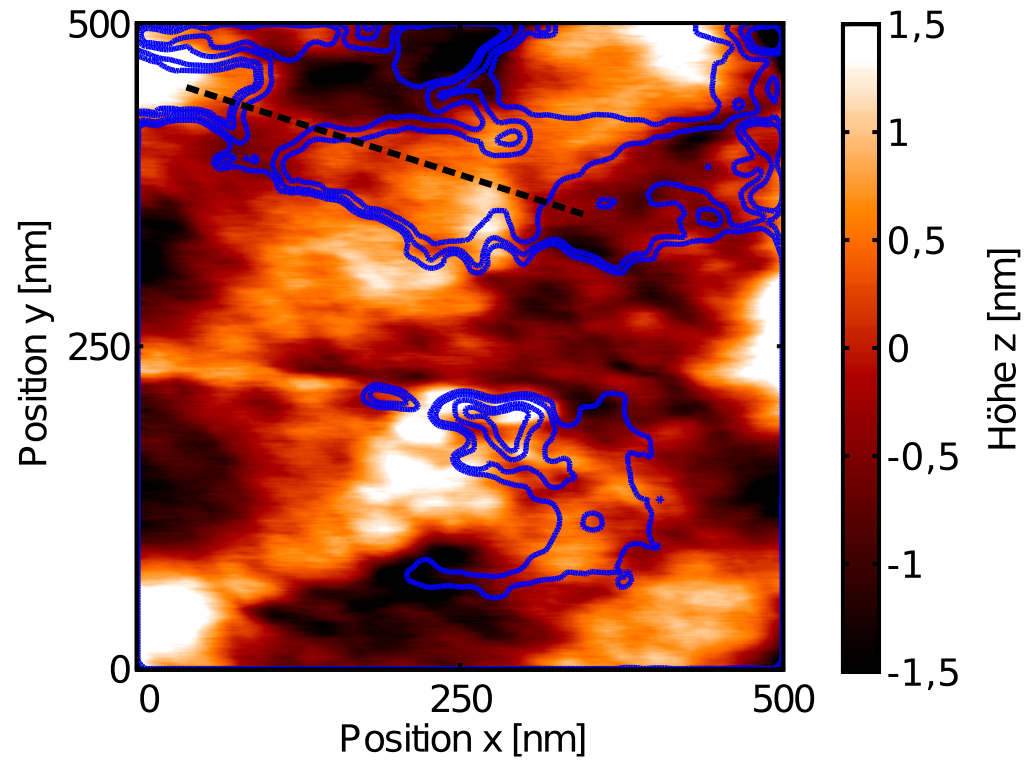

Abbildung 4.22: Topographie zur Stromkarte in Abb. 4.21. Die Konturlinien kennzeichnen die gleichen Leitfähigkeitsbereiche wie in der Stromkarte, das heißt bei den im nachfolgenden Histogramm (siehe Abb. 4.23) markierten Werten. Man erkennt im Vergleich mit Abb. 4.21 eine deutliche Korrelation zwischen der Topographie und dem Schaltverhalten. 
Region. Die beiden oberen Pulse schalteten ebenfalls sehr großflächige Bereiche, welche trotz des Abstandes von etwa $250 \mathrm{~nm}$ zwischen den Pulsorten sogar zu einem gemeinsamen isolierenden Gebiet verschmolzen sind.

Die Farbkodierung der Stromkarte wurde in diesem Fall von der bisherigen Darstellung abweichend gewählt, um eine besondere Eigenart dieser Messung hervorzuheben: Man erkennt in den isolierenden Regionen eine Segregation in Unterbereiche verschiedener, anscheinend diskreter Leitfähigkeiten. Die meisten Gebiete weisen dabei noch eine gegenüber dem Ausgangszustand stark erhöhte Leitfähigkeit auf.

Die einzelnen Regionen erscheinen sehr scharf gegeneinander abgegrenzt. Darüberhinaus weisen mehrere räumlich von einander getrennte Gebiete offenbar die gleiche Leitfähigkeit auf - besonders gut erkennbar im Falle der beiden gelb dargestellten Bereiche. Die leichte Verschiebung der unteren Region ins Grünliche, das heißt zu niedrigeren Strömen hin, geschieht bei näherer Betrachtung zeilenweise und ist somit offensichtlich als Artefakt, wahrscheinlich durch eine zeitweilige Kontamination der Cantileverspitze, einzuordnen.

Diese Messung zeigt also eindeutig das Vorliegen mehrerer diskreter Widerstandswerte. Zur Verdeutlichung ist die Häufigkeitsverteilung der gemessenen Stromwerte in Abb. 4.23 noch als Histogramm wiedergegeben. Der bei weitem dominierende Beitrag für die noch metallische Matrix wurde, ebenso wie der kleinere hochisolierende Anteil, dessen Werte sich zu einem Balken vereinigen, oben abgeschnitten. Eine lineare Auftragung der Häufigkeit erlaubt eine bessere Darstellung der restlichen, in ihrer Höhe vergleichbaren Peaks. Die Farbkodierung des Histogramms wurde entsprechend der in Abb. 4.21 gezeigten Stromkarte gewählt.

Man erkennt deutlich das Vorliegen je eines Peaks bei etwa $2 \mathrm{nA}$ und eines bei $24 \mathrm{nA}$. Letzterer ist - wahrscheinlich durch die bereits erwähnten Spitzenartefakte - mit einer kleinen Aufspaltung behaftet. Bei ungefähr $12 \mathrm{nA}$ ist ferner eine im Vergleich sehr breite Häufung erkennbar. Diese Verbreiterung ist eventuell auf Ausläufer von den größeren Beiträgen oder die Aufzeichnung von Zwischenwerten an den Zonenrändern zurückzuführen; möglicherweise handelt es sich aber auch um eine Überlagerung mehrerer kleinerer Peaks.

Die schwarze Fitkurve entsteht durch Summation der vier rot dargestellten gaufförmigen Verteilungen und nach Abzug des kleinen, hier gestrichelt eingetragenen Untergrunds. Die Peakpositionen wurden durch Pfeile kenntlich gemacht. Die vertikalen Linien kennzeichnen manuell gewählte ungefähre Grenzwerte, die in Abb. 4.21 und Abb. 4.22 als Konturlinien ergänzt wurden. Hierdurch wird eine gewisse Korrelation zwischen der Stromkarte und der Topographie ersichtlich: Die in der Stromkarte gelb und hellblau dargestellten, also metallischeren Gebiete liegen bevorzugt in Senken, die durch dunklere Blautöne wiedergegebenen isolierenden Bereiche entsprechend ausschließlich auf Erhebungen. ${ }^{\mathrm{i}}$

Zur Veranschaulichung dieser Korrelation ist in Abb. 4.24 ein Höhen- und Stromprofil entlang des in den Farbkarten eingetragenen Pfades dargestellt. Man erkennt deutlich das gegenläufige Verhalten; nur in Senken, wo die Höhenlinie ein bestimmtes Niveau unterschreitet, wurden große Stromwerte gemessen.

${ }^{\mathrm{i}}$ Der durch die Vormessungen erzwungene, flächendeckend metallische Zustand ist natürlich für alle Höhenwerte präsent und muss bei dieser Betrachtung ignoriert werden - er erweist sich in den Tälern der Topographie allenfalls als etwas stabiler. 


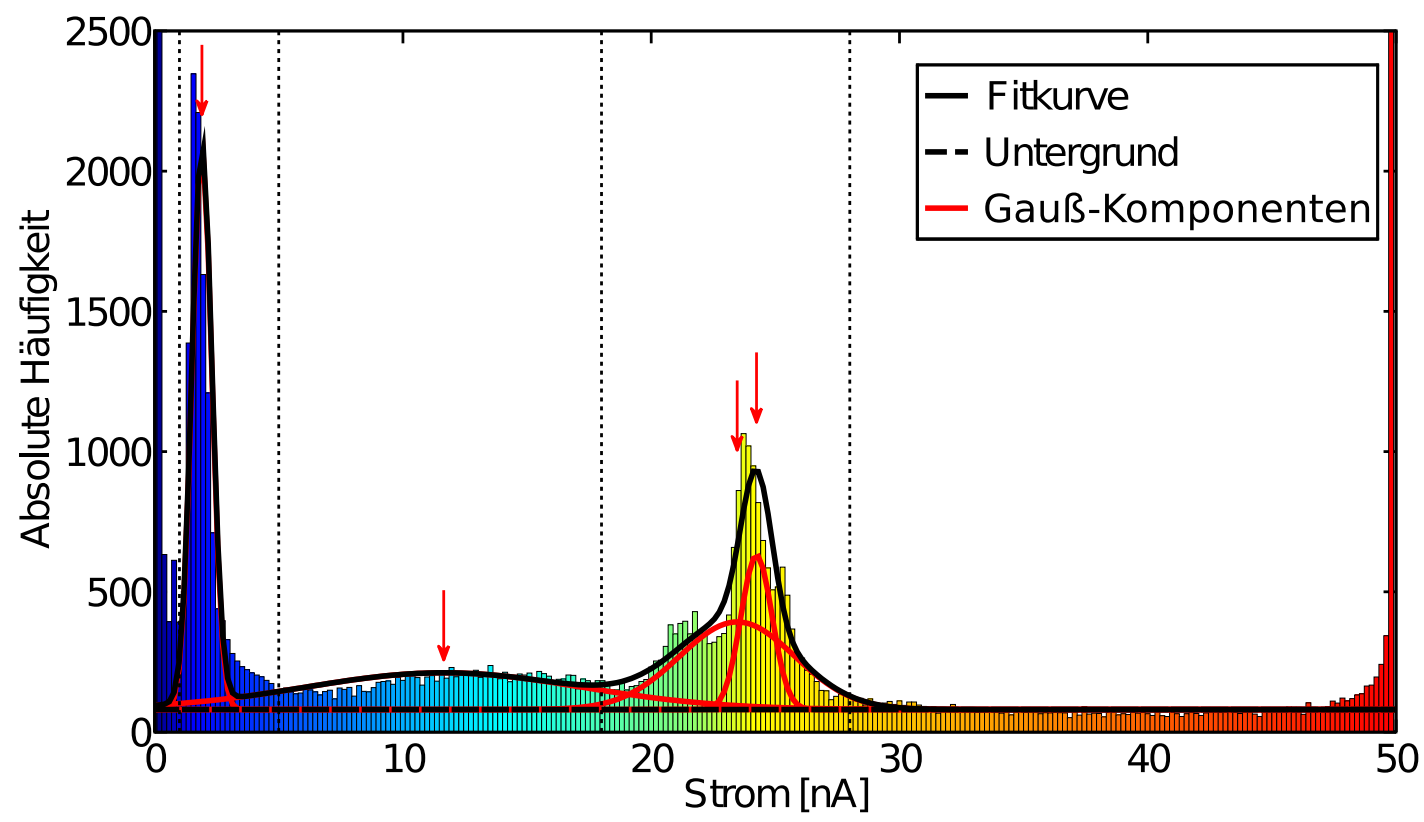

Abbildung 4.23: Histogramm zu Abb. 4.21. Die Häufigkeitsverteilung der mittleren Stromwerte lässt sich durch eine Summe der vier in rot eingetragenen Gaußfunktionen annähern. Die zugehörigen Parameter sind in Tab. $4.1 \mathrm{zu}$ finden und die jeweiligen Peakpositionen durch die Pfeile markiert. Die Gesamtverteilung ist schwarz dargestellt; die gestrichelte horizontale Linie kennzeichnet einen zuvor subtrahierten Untergrund. Die vertikalen Linien entsprechen den manuell festgelegten Peakgrenzen, welche in Abb. 4.21 und Abb. 4.22 als Konturlinien eingetragen sind. Die dominanten Peaks nahe 0 nA und bei $50 \mathrm{nA}$, der Sättigungsschwelle des Gerätes, wurden abgeschnitten.

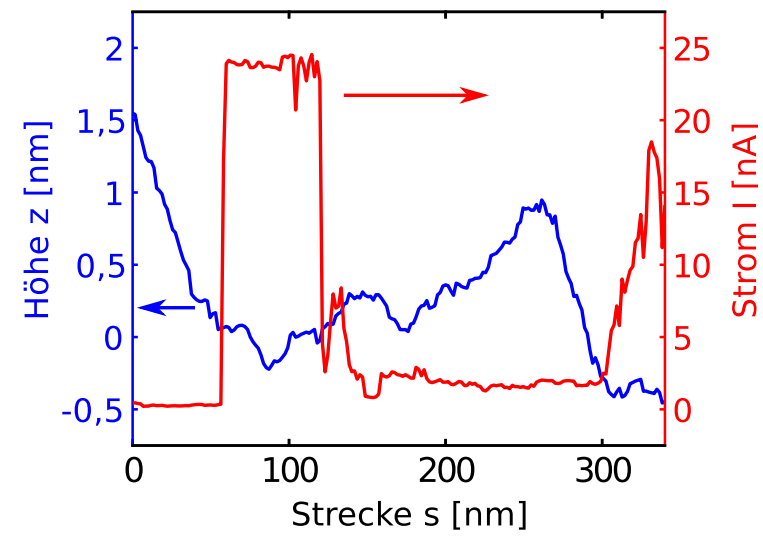

Abbildung 4.24: Korrelation zwischen lokaler Leitfähigkeit und Höhenprofil. Die Stromund Höhenverläufe entlang des in Abb. 4.21 und Abb. 4.22 markierten Pfades zeigen gegenläufiges Verhalten: Metallische Bereiche finden sich in Senken der Topographie. 
Tabelle 4.1: Erwartungswerte $\mu$, Standardabweichungen $\sigma$ und relative Gewichtungen $p$ der in die Fitfunktion zum Histogramm Abb. 4.23 einfließenden Gaußverteilungen.

\begin{tabular}{ccc}
\hline$\mu[\mathrm{nA}]$ & $\sigma[\mathrm{nA}]$ & $p$ \\
\hline 1,9 & 0,2 & 0,31 \\
11,6 & 31,2 & 0,28 \\
23,5 & 5,4 & 0,28 \\
24,2 & 0,4 & 0,13 \\
\hline
\end{tabular}

\subsection{Zusammenfassung}

Es wurden dünne, epitaktische Manganatfilme verschiedener Lochdotierungen und B-Ionenradien mittels der C-AFM-Methode untersucht. Die Ergebnisse der durchgeführten Standardcharakterisierung, welche von der guten Kristallqualität der Proben zeugt und die typischen grundlegenden Bulk-Manganateigenschaften des thermisch induzierten Metall-IsolatorÜbergangs nahe der Curie-Temperatur und den Kolossalen Magnetowiderstandseffekt zeigt, wurde exemplarisch für eine der Proben dargestellt.

Allen untersuchten Schichten gemein ist das Widerstandsschalten bei Anlegen von Spannungen an die C-AFM-Spitze. Folgende Erkenntnisse wurden dabei gewonnen:

- Positive Spannungen erzeugen an allen untersuchten Probensystemen stets den niederohmigen LRS, negative dagegen den isolierenden HRS - das Schalten verläuft bipolar. Auch eine geringe Anzahl von Messungen bei tieferen Temperaturen $T<T_{\mathrm{c}}$ zeigen das gleiche qualitative Verhalten und die dieselbe Polarität.

- Es ist eine scharfe Schwellspannung von mindestens $U_{\mathrm{c}} \approx 3 \mathrm{~V}$ vorhanden.

- Das Schalten ist prinzipiell wiederholbar, meistens wird jedoch der extrem hochisolierende Ausgangszustand nicht wieder exakt reproduziert; auch der LRS kann Alterungseffekte bei wiederholtem Schalten aufweisen.

- Unter angelegter Pulsspannung können sowohl kleine, scharf begrenzte leitfähige Regionen von wenigen Zehn Nanometern Durchmesser, als auch sich über große Flächen ausbreitendes Wachstum beobachtet werden. Es können sowohl metallische Flecken in einer isolierenden Matrix als auch umkehrt isolierende Inseln in metallischer Umgebung generiert werden.

- Vereinzelt ist die Entstehung von Satellitenflecken zu beobachten, das heift Regionen, die relativ weit von der eigentlichen Pulsposition entfernt sind, aber trotzdem in den LRS umgeschaltet werden.

- Gelegentlich entstehen zum Teil drastische Oberflächenmodifikationen beim Anlegen ausschließlich positiver Spannungspulse - dieses Verhalten beruht vermutlich auf einer Schädigung der Oberfläche und der C-AFM-Spitze. 
- Die Ausbreitung metallischer Regionen vollzieht sich umso schneller, je größer die erzeugende Pulsspannung ist. Es scheint eine lineare Abhängigkeit des Domänenradius von der Amplitude vorzuliegen.

- Das Wachstum des Domänenradius folgt einer logarithmischen Zeitentwicklung. Die ebenfalls beobachtete zeitliche Rückbildung metallischer Bereiche erfolgt mit sehr verschiedenen Geschwindigkeiten - von quasi stabilen Konfigurationen bis hin zu einer sehr raschen Relaxation. Auch im Falle einer aufgezeichneten sehr langsamen Auflösung einer leitfähigen Domäne ließ sich die Größenabnahme durch ein logarithmisches Zeitgesetz beschreiben.

- Pulsfolgenexperimente zeigen eine starke Abhängigkeit der resultierenden Leitfähigkeit nicht nur von der Amplitude und Dauer der Pulse, sondern auch von dem zeitlichen Abstand zwischen kurzen Pulsen: Bei zu großen Verzögerungen kann keine Erhöhung der Leitfähigkeit erzielt werden.

- Die Details des resistiven Schaltens sind auf der Nanometerskala - auch von den häufig verursachten Oberflächenschädigungen absehend - mit großen statistischen Schwankungen behaftet. Dies betrifft die Schwellspannung, die erzielbaren Widerstandseffekte, die Domänenausbreitung und ihre zeitliche Stabilität.

- Eine mit nur zwei zusätzlichen Monolagen LMO bedeckte LCMO-Schicht zeigt zumindest auf einem signifikanten Oberflächenanteil ein von Beginn an metallisches Verhalten mit vergleichsweise homogener Leitfähigkeitsverteilung.

- An einer LSMO-Schicht konnte das anscheinend über mehrere diskrete Widerstandszustände verlaufende Schalten in Form ausgedehnter, zum Teil räumlich voneinander getrennter Regionen mit homogener Leitfähigkeit beobachtet werden. Es liegt dabei eine starke Korrelation mit der Topographie vor: Erhebungen zeigen vorwiegend höhere Widerstände als ihre Umgebung. 



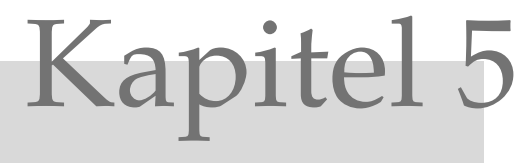

Diskussion

Elektrisch stimulierte Widerstandsänderungen sind zwar ein häufiges Phänomen oxidischer Systeme, der zugrundeliegende mikroskopische Mechanismus ist aber bei den hier untersuchten Manganaten bisher unbekannt.

Die im vorigen Kapitel beschriebenen C-AFM-Messungen können wertvolle Beiträge zur Aufschlüsselung des Schalteffektes leisten. Basierend auf den allgemeinen Charakteristika des Effektes wird im ersten Abschnitt dieses Kapitels ein phänomenologisches Modell entworfen, in welchem sich die Widerstandsänderung als Folge einer lokalen Strukturänderung ergibt. Die genauen mikroskopischen Vorgänge können dabei natürlich nicht ansatzweise herausgearbeitet werden; es handelt sich nur um eine Arbeitshypothese. Trotz seiner detailliert erscheinenden Formulierung dient das qualitative Modell nur als Illustration, wie man sich eine remanente, elektrisch induzierte Modifikation der Struktur als Auslöser des Widerstandsschaltens prinzipiell vorstellen könnte. Es werden außerdem knapp die wichtigsten offenen Fragen, bei denen das vorgestellte qualitative Modell noch zu kurz greift, angesprochen und mögliche Ergänzungen aufgezeigt.

In den darauffolgenden Abschnitten wird erläutert, welche konzeptionellen Schwierigkeiten sich für die vorliegenden Messergebnisse bei einer alternativen Beschreibung im Rahmen der in der Fachliteratur etablierten Modelle ergeben. Der Schwerpunkt liegt dabei auf den Unzulänglichkeiten des in der gegenwärtigen Debatte favorisierten Szenarios der Elektromigration von Sauerstoffionen als zentralem Prozess. Dem in diesem Zusammenhang wichtigen Aspekt großer Stromdichten und Heizeffekte ist ein eigener Abschnitt gewidmet, welcher auch einer Einordnung der beobachteten Oberflächenmodifikationen enthält.

Es folgt die Diskussion der komplexeren Eigenschaften des Schalteffektes; sie helfen bei einer Entscheidungsfindung über die bessere Eignung des Sauerstoffmodells oder der strukturellen Hypothese für die vorliegenden Resultate. Dabei wird zu erst die Wachstumsdynamik der metallischen Domänen im elektrischen Feld als auch ihre zeitliche Rückbildung besprochen, welche eine Abwägung des strukturellen Modells als Arbeitshypothese gegen das ionische Bild ermöglicht. Im Anschluss werden der Einfluss der Terminierung mit undotiertem Lanthanmanganat auf die Oberflächenleitfähigkeit und die Beobachtung anscheinend diskreter, mit der Topographie korrelierter Leitfähigkeitsplateaus an der Oberfläche eines glatten LSMO-Filmes im Kontext der beiden Modelle diskutiert. 


\subsection{Modell: Elektrisch induzierter struktureller Übergang}

In Kapitel 2 wurden bereits die gängigen Erklärungsversuche für elektrisch induzierte Schalteffekte der hier untersuchten Art vorgestellt, beispielsweise das Bild der Elektromigration von Sauerstoffleerstellen oder das Modell einer durch Ladungsinjektion kontrollierbaren Schottky-Barriere. Im Rahmen dieser Arbeit wurde jedoch ein qualitatives Modell entwickelt, welches einen alternativen Erklärungsansatz für das beobachtete Schaltverhalten bietet und zusammen mit den zuvor gezeigten, experimentellen Resultaten publiziert wurde. ${ }^{[139]}$ Dieses phänomenologisch motivierte Bild basiert auf der Annahme eines spannungsinduzierten strukturellen Überganges, welcher die Widerstandsänderung hervorruft.

Bevor die Modellbildung im Detail ausgeführt wird, wollen wir diesen Ansatz im Folgenden jedoch zuerst motivieren und dann durch eine Analyse der Messergebnisse seine zentralen Thesen herausarbeiten.

\subsubsection{Motivation}

Die Bedeutung des komplexen Wechselspiels struktureller, elastischer, magnetischer und elektrischer Eigenschaften für die Phänomenologie perowskitischer Manganate ist seit langem anerkannt und spätestens seit der Entdeckung des kolossalen Magnetowiderstandseffektes Gegenstand intensiver Forschung. Diese Bemühungen zeigten für eine Vielzahl an Materialien strukturelle Phasenseparation ohne gleichzeitige chemische Entmischung. Renner et al. fanden außerdem an der Oberfläche von $\mathrm{Bi}_{0,24} \mathrm{Ca}_{0,76} \mathrm{MnO}_{3}$ eine Koexistenz strukturell und elektrisch verschiedener Bereiche. ${ }^{[70]}$

Manganate sind elektronisch von hochkorrelierter Natur und besitzen zum Teil eine starke Elektron-Phonon-Kopplung. ${ }^{[49]}$ Eine separate Beschreibung von Eigenschaften des Elektronen- und des Gittersystems ist daher oftmals unmöglich oder versagt zumindest bei der Wiedergabe phänomenologischer Details. Schon das Vorliegen nachweisbar polaronischer Ladungsträger zeigt, dass jegliche Transportphänomene eigentlich unter Berücksichtigung der Einflüsse des Ionengitters behandelt werden müssten. Jede strukturelle Änderung, sei sie thermisch, durch Magnetfelder, chemischen oder externen Druck oder andere Einflüsse hervorgerufen, äußert sich demzufolge auch durch eine Änderung des elektrischen Widerstandsverhaltens.

Es erscheint daher durchaus naheliegend, die Ursache des in der vorliegenden Abhandlung untersuchten Widerstandsschaltens in elektrischen Feldern ebenfalls in einer strukturellen Veränderung des Materials zu suchen. Diese Idee ist im Grundsatz nicht neu: Neben den Arbeiten von Esseling ${ }^{[107]}$ und Moshnyaga et al. ${ }^{[108]}$, in welchen bereits die Möglichkeit eines strukturellen Mechanismus für das Widerstandsschalten nanokolumnarer LSMO-Filme diskutiert wird, finden sich in der Literatur noch weitere Indizien für einen strukturellen Übergang als Ursache oder zumindest bedeutende Komponente des Effektes.

Für PCMO sei hier vor allem auf die Arbeiten von Jooss et al. verwiesen, wo es gelang, die mit dem Schalten einhergehende Bildung und Zerstörung von Polaronenordnungen mittels hochauflösender Transmissionselektronenmikroskopie abzubilden. ${ }^{[56,106]}$ Neben solchen Änderungen der kollektiven Drehungs- und Verkippungsmuster der $\mathrm{MnO}_{6}$-Oktaeder wurde von Hsu et al. auch auf die Möglichkeit einer Schwächung beziehungsweise Stärkung des Jahn-Teller-Effektes durch Spannungspulse hingewiesen. ${ }^{[05]}$ Allgemeiner äußerten Driscoll 
et al. für Vanadiumdioxid, welches einen Isolator-Metall-Übergang zeigt, die Vermutung, dass eben die bekannte Phasenkonkurrenz ${ }^{\mathrm{a}}$ für die memristiven Eigenschaften des Materials verantwortlich zeichnet. ${ }^{[104]}$

Auch die in dieser Arbeit untersuchten Materialien zeigen nachweislich eine subtile Balance verschiedener Widerstandszustände, die - neben der Temperatur - durch eine ganze Reihe externer Stimuli entscheidend beeinflusst werden kann. Von dieser Tatsache ausgehend erscheint es nur naheliegend, das remanente Widerstandsschalten als eine weitere Ausprägung dieses Wettstreites verschiedener struktureller Konfigurationen aufzufassen. In einer konkreten mikroskopischen Beschreibung muss neben materialspezifischen Eigenschaften natürlich auch der besondere Einfluss der Oberfläche mit einbezogen werden.

Aktuelle theoretische Arbeiten auf dem Gebiet der elektronisch hochkorrelierten Systeme, wie beispielsweise die von Leonov et al. durchgeführten Rechnungen für $\mathrm{KCuF}_{3}$ und $\mathrm{LaMnO}_{3}$, unterstreichen die starke Abhängigkeit der strukturellen Eigenschaften eines Materials von elektronischen Korrelationseffekten. Diese Rechnungen auf Basis der dynamischen Molekularfeldtheorie (,Dynamic Mean Field Theory", DMFT) belegen, dass eine Modifikation der Struktur durch elektrische Felder, welche die Orbitalordnung beeinflussen, durchaus denkbar ist. Aktuelle Studien zeigen, dass sogar das strukturelle Phasendiagramm von Eisen - siehe Abb. 5.1 - nur unter Einbezug elektronischer Korrelationen

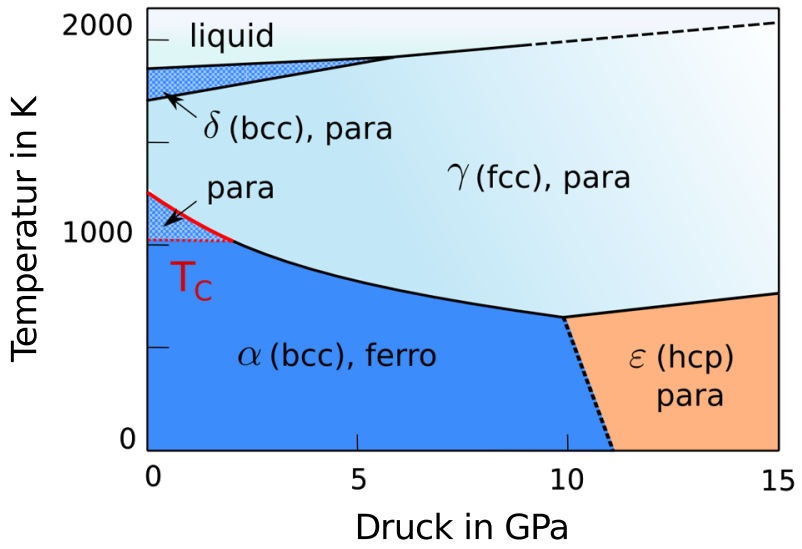

Abbildung 5.1: Magnetisches und strukturelles Phasendiagramm von Eisen (aus [140]). Der strukturelle Übergang vom $\alpha$ - zum $\gamma$-Eisen findet erst deutlich oberhalb der Curie-Temperatur $T_{\mathrm{C}}$ statt. Ein Verständnis ist nur unter Einbezug elektronischer Korrelationen möglich. korrekt erhalten werden kann. ${ }^{[140]}$

In der nun folgenden phänomenologischen Modellentwicklung kann natürlich kein vergleichbares und belastbares Bild entworfen werden, welches der Komplexität der mikroskopischen Details dieser komplizierten Materialien auch nur ansatzweise gerecht wird. Stattdessen soll auf anschaulichem Niveau ein rein hypothetischer Mechanismus herausgearbeitet werden. Diese alternative Beschreibung durch ein nicht-ionisches Szenario soll dabei den Indizien für die Beteiligung einer strukturellen Komponente nachkommen und vor allem als Denkanstoß für die Konstruktion einer realistischen Darstellung wahrgenommen werden.

\footnotetext{
${ }^{a}$ In diesem Fall wurde auf die Möglichkeit hingewiesen, mittels lokaler Joulescher Heizeffekte entlang perkolativer Strompfade bleibende Widerstandsänderungen zu induzieren.
} 


\subsubsection{Vorüberlegungen}

Bevor die eigentliche Modellbildung erfolgt, sollen einige Vorüberlegungen angestellt werden, um die zentralen Annahmen des Modells zu begründen. Dabei werden die experimentellen Befunde aufgegriffen und, wo nötig, durch aus der Literatur bekannte Ergebnisse ergänzt.

\section{Generelle Einordnung der Messergebnisse}

Die im Rahmen dieser Arbeit gewonnenen experimentellen Ergebnisse stellen in vielerlei Hinsicht wertvolle Ergänzungen zum bisher veröffentlichten Wissensstand dar. Die C-AFMMethode liefert interessante Einsichten in die räumlichen Eigenschaften der Schaltprozesse und ihre Korrelationen mit der Topographie.

Die meisten bisher publizierten Arbeiten zum resistiven Schalten beziehen sich auf MIMSchichtsysteme. Diese sind aus anwendungsorientierter Sicht als prototypische Bauelemente durchaus reizvoll, lassen aber allenfalls mesoskopische Messungen des gesamten Systems zu. Die Verwendung der leitfähig beschichteten AFM-Spitze als mobile Topelektrode ist hingegen besonders geeignet, um die mikroskopische, resistive Antwort vor allem der Oberfläche des Manganatfilmes selbst zu beobachten. Der Einfluss präparativer Details bei der Kontaktierung auf die Grenzfläche zur Elektrode spielt keine Rolle mehr, es ist jedoch gegebenenfalls mit Kontaminationen der Oberfläche und Alterung der Sonden zu rechnen.

Bei einer Kontaktierung durch großflächige Elektroden ist ferner nicht a priori klar, ob das gemessene Schaltverhalten repräsentativ für die gesamte Grenzfläche ist. ${ }^{\mathrm{b}}$ Selbst nanoskalige Inhomogenitäten werden mit der C-AFM-Methode stattdessen direkt abgebildet.

$\mathrm{Zu} \mathrm{SrTiO} 3$ wurden schon einige C-AFM-Studien bezüglich des resistiven Schaltens veröffentlicht, die das filamentäre Schaltszenario unterstützen. ${ }^{[8,94,141,142]}$ Sie wurden im Kontext der Sauerstoffmigration diskutiert und die Einflüsse von diffusionsfördernden Strukturdefekten untersucht.

Für Manganate fand die C-AFM-Methode hingegen nur sehr begrenzten Einsatz. Die größten Parallelen finden sich zu den Experimenten von Chen et al.; sie zeigten für LSMO granulares Schalten auf der Nanoskala. ${ }^{[9]}$ Dabei wurden, ähnlich den hier dargestellten Resultaten, metallische Bereiche größerer Ausdehnung, etwa $40 \ldots 160 \mathrm{~nm}$, mit der AFM-Spitze erzeugt. Ihre Form korreliert mit der durch Inselwachstum geprägten Oberflächenmorphologie. Im Gegensatz zu den hier untersuchten Systemen präsentierte sich die Leitfähigkeit der Proben jedoch in einem deutlich inhomogeneren Ausgangszustand.

C-AFM-Ergebnisse von Manganaten in der hier vorliegenden Qualität sind zuvor nicht veröffentlicht worden. Insbesondere die Abbildung der Spannungs- und Pulsdauerabhängigkeit des Domänenwachstums auf der Nanometerskala ist ein Novum auf diesem Gebiet. Phänomenologisch bestehen hier allerdings gewisse Parallelen zu den von Paruch et al. und Tybell et al. durchgeführten C-AFM-Messungen, welche dem ferroelektrischen Schalten von $\mathrm{Pb}\left(\mathrm{Zr}_{0.2} \mathrm{Ti}_{0.8}\right) \mathrm{O}_{3}$ gewidmet sind. ${ }^{[143,144]}$ Das von ihnen demonstrierte Kriechverhalten von ferroelektrischen Domänenwänden - im weiteren Sinne ein struktureller Übergang -

\footnotetext{
${ }^{\mathrm{b}}$ Bei inhomogenen Systemen wie Manganaten könnte ja bespielsweise bereits ein kleiner Kontaktflächenanteil, welcher leichter zu schalten ist, das Widerstandsverhalten der MIM-Struktur dominieren. In diesem Falle würde offensichtlich nicht einmal unbedingt das über die Probe gemittelte elektrische Verhalten erfasst werden.
} 
ähnelt dem hier beobachteten stark. Dieser Aspekt wird in Abschnitt 5.4 aufgegriffen und detailliert untersucht.

\section{Rolle der ,treibenden Kraft“"}

Von großer Bedeutung ist die Frage, welche physikalische Größe als Auslöser für das resistive Schalten angesehen werden muss. Ob der Schaltprozess durch das Überschreiten einer Schwellspannung oder einer kritischen Stromdichte ausgelöst wird, ist nämlich oftmals nur schwer zu beantworten. Die Unterscheidung ist deshalb schwierig, weil die Stromdichte sich ja bei konstantem Widerstand proportional zur angelegten Spannung erhöht.

In unserem Fall ist jedoch angesichts der enormen Effektgröße, also der starken Widerstandsänderung auf lokaler Skala, eine Aussage möglich: Es besteht zumindest eine ungefähr eingehaltene Symmetrie in der kritischen Spannung $U_{\mathrm{c}}$, das Schalten zwischen HRS und LRS und zurück findet also bei positiven und negativen Spannungen des etwa gleichen Betrages statt. ${ }^{\mathrm{c}}$ Der Strom beträgt unmittelbar vor dem ersten Schalten vom Ausgangszustand in den LRS höchstens einige zehn Pikoampere, selbst mit $0,1 \mathrm{nA}$ entspricht dies bei einer Kontaktfläche von mindestens $A_{\text {Kontakt }}=\pi a^{2}=3,8 \mathrm{~nm}^{2}$ einer Stromdichte von höchstens einigen $10^{3} \mathrm{~A} / \mathrm{cm}^{2}$ (siehe

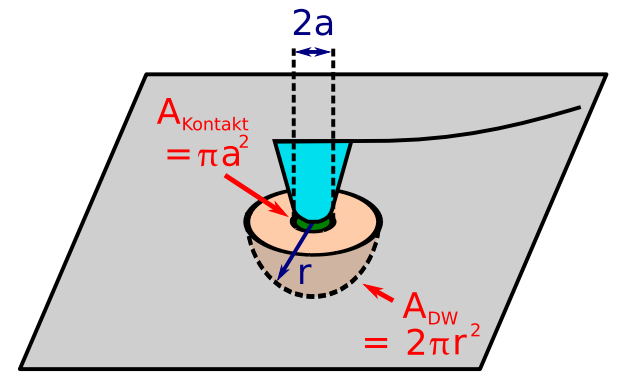

Abbildung 5.2: Kontaktfläche an der Oberfläche und näherungsweise halbkugelförmige Domänenwand (schematisch).

Abb. 5.2). Beim entgegengesetzten Schalten in den HRS fließen wahrscheinlich Ströme im Mikroampere-Bereich; wenn wir beispielsweise eine halbkugelförmige Domäne von $r=20 \mathrm{~nm}$ Radius annehmen, so ergibt sich an der Domänenwand $A_{\mathrm{DW}}=2 \pi r^{2} \approx 2400 \mathrm{~nm}^{2}$ hingegen eine Stromdichte von einigen $10^{4} \mathrm{~A} / \mathrm{cm}^{2}$. Das Schalten beginnt demnach in Hin- und Rückrichtung bei gleicher kritischer Spannung, aber bei Stromdichten, die sich um mindestens eine Größenordnung unterscheiden. ${ }^{\mathrm{d}}$

An der viel kleineren Kontaktfläche zwischen Spitze und Probe liegen beim Zurückschalten sogar noch um mehrere Zehnerpotenzen stärkere Stromdichten vor. Falls also die Stromdichte der Auslöser wäre, so würde man - im Gegensatz zu den vorgestellten Ergebnissen das Einsetzen eines graduellen Zurückschaltens in den HRS hier bereits im negativen Millivoltregime erwarten können.

Streng genommen widerspricht bereits die beobachtete Pulsdauerabhängigkeit einer kritischen Stromdichte: Während der Ausbreitung einer metallischen Domäne unter konstant anliegender Spannung nimmt ihre Oberfläche immer weiter zu, die Stromdichte verhält sich reziprok. Nur unter gleichzeitiger Erhöhung der Spannung dürfte es also zu einem Wachstum kommen.

\footnotetext{
${ }^{\mathrm{c}}$ Auch wenn andere Ergebnisse zeigten, dass sich der isolierende Zustand manchmal auch ohne negative Spannung regenerieren kann, ist das wenn auch nur gelegentliche Auftreten von positiver und negativer Schwellspannung ähnlicher Größe nur schwerlich als Zufall anzusehen.

${ }^{\mathrm{d}}$ Die maximalen Stromdichten, welche immer an der Kontaktfläche $A_{\text {Kontakt }}$ auftreten, werden unter 5.3 noch detaillierter untersucht.
} 
Auch die These der Leitfähigkeitsänderung in Folge einer Ladungsinjektion, wie sie zum Beispiel von Rozenberg et al. vorgeschlagen wurde, ${ }^{[99]}$ erscheint in diesem Kontext eher unrealistisch: Zwar können bei Spannungen unterhalb von $U_{\mathrm{c}}$ im hochisolierenden Ausgangszustand gelegentlich kurzfristige, kapazitive Lade- und Entladeströme beobachtet werden (nicht gezeigt), die aufintegrierte Ladungsmenge ist allerdings verschwindend im Vergleich zu den Ladungsmengen, welche im LRS transportiert werden.

Es kann also für die gezeigten Messungen mit großer Sicherheit davon ausgegangen werden, dass die Spannung oder eine ihr näherungsweise proportionale Größe wie die elektrische Feldstärke - nicht aber die Stromdichte - den Schaltprozess initiiert.

\section{Größenordnung der lokalen Felder}

Betrachten wir die Spannung nun als treibende Größe des Effektes, so können wir ihre Größenordnung in dem Bereich, wo sich die Schaltprozesse abspielen, grob abschätzen. Der in der Messung erfasste Gesamtwiderstand bezieht sich auf das System aus dem großflächigen, geerdeten Leitsilberkontakt, der Probe und dem als mobile Topelektrode fungierenden Cantilever.

Die erzielten Widerstandsänderungen erstrecken sich über bis zu etwa drei Größenordnungen (vergleiche Abschnitt 4.4). Das bedeutet tatsächlich, dass auch fast die gesamte außen angelegte Spannung an dem schaltenden Bereich abfällt - zumindest bis der Wechsel in den LRS erfolgt ist.

Aus den Stromkarten ist ferner klar ersichtlich, dass sich die Widerstandsänderung oftmals auf Bereiche von nur wenigen zehn Nanometern Durchmesser beschränkt. Für $U_{c} \approx 3 \mathrm{~V}$ und $r \approx 10 \mathrm{~nm}$ ergibt sich damit bereits eine sehr große elektrische Feldstärke von $|E|=U / r=3 \cdot 10^{8} \mathrm{Vm}^{-1}$.

Bedenkt man außerdem, dass die Domänen nicht als Ganzes abrupt umschalten, sondern sich nach außen hin ausbreiten, so muss man eigentlich explizit auf die Rolle der spannungsgetrieben propagierenden Grenzfläche eingehen. Der Domänenwand muss ein eigener, beträchtlicher Widerstandsbeitrag und eine noch größere lokale Feldstärke zugeschrieben werden. Diese Vorstellung wird auch in Hinsicht auf das Zurückschalten in den HRS den realen Verhältnissen wahrscheinlich eher gerecht - der Spannungsabfall über die gesamte Domäne ist im LRS ja um Größenordnungen reduziert. Tatsächlich fungiert die metallische Region vermutlich nur als eine Art „Zuleitung“ zum Ort der größten Feldstärke, der Phasengrenze. Die Domänengröße ist im Übrigen sehr klein im Vergleich zum Kontaktabstand, sodass sich auch keine all zu schnelle Abnahme der lokalen Feldstärke ergeben sollte.

Aus einer solch starken Inhomogenität des Widerstands im Bereich der Domänenwand ergibt sich außerdem eine abrupte Änderung des elektrischen Feldes beziehungsweise ein starker lokaler elektrischer Feldgradient.

\section{Bedeutung der Schaltschwelle}

Die in den Messungen ersichtliche, harte Spannungsschwelle, unterhalb derer kein Widerstandsschalten stattfindet, muss als Hinweis auf eine systemimmanente Energiebarriere verstanden werden, welche beim Umschalten zwischen den beiden Widerstandszuständen überwunden werden muss. Beliebig kleine Spannungen können, auch bei entsprechend 
längerer Pulszeit, keine Widerstandsänderungen vom HRS zum LRS hervorrufen. Abb. 5.3 skizziert ein entsprechendes Zweiniveaumodell bezüglich einer verallgemeinerten, strukturellen Koordinate.

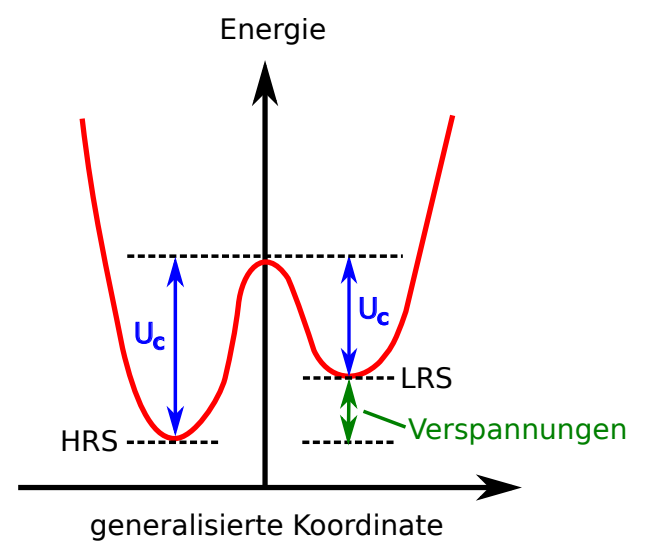

Abbildung 5.3: Zweiniveaumodell der Widerstandszustände. Die Energiebarriere kann mittels der Spannung $U$ überwunden werden. Der HRS ist niedriger anzusiedeln, durch inhomogene Verspannungen kann die Differenz der beiden Zustände lokal variieren.

Aufgrund der zumindest ungefähr bestehenden Symmetrie zwischen den Schaltspannungen in beiden Richtungen müssen die Widerstandszustände selbst von ähnlicher Energiedichte sein. Die Barrierenhöhe ist hier also vermutlich groß im Vergleich zur Energiedifferenz der beiden Widerstandszustände. Die Beobachtung einer selbstständigen Rückbildung des HRS (siehe Abb. 4.15) ohne anliegende Spannung deutet jedoch darauf hin, dass die Stabilität des energetisch ungünstigeren LRS auch oftmals einem zusätzlichen pinning, also dem Haften der Domänenwände an Defekten - insbesondere der Oberflächenmorphologie geschuldet ist.

\section{Widerstandszustände}

Der wohlbekannte starke Einfluss der Gitterstruktur auf die elektronischen Eigenschaften legt nahe, die beiden Widerstandszustände mit den zwei für die behandelten Manganatsysteme relevanten Modifikationen der Perowskitstruktur zu identifizieren. Wir gehen also von einer höhersymmetrischen, pseudokubischen Anordnung wie der $R \overline{3} c$-Struktur für die metallische Phase aus. Die konkurrierende Pnma-Struktur hat einen wesentlich größeren Widerstand und sollte entsprechend als der hochohmigen Phase ähnelnd angenommen werden.

Im Rahmen dieser Arbeit sind keine kristallographischen Experimente durchgeführt worden, die eine tatsächliche Strukturaufklärung der metallischen und isolierenden Bereiche gestatten. Diese wären allein wegen der geringen Schichtdicke und auch der kleinen lateralen Ausdehnung der leitfähigen Bereiche äußerst anspruchsvoll. Daher ist es grundsätzlich auch durchaus möglich, dass andere kristallographische Raumgruppen realisiert sind, dies gilt insbesondere nahe der Oberfläche. Da also keine verlässlichen Informationen über die genaue strukturelle Symmetrie der untersuchten Manganatoberflächen vorliegen, werden die konkurrierenden Phasen hier nur als höhersymmetrisch beziehungsweise niedersymmetrisch bezeichnet, können aber als Arbeitshypothese mit den Eigenschaften der $R \overline{3} c$ - und 
Pnma-Struktur gedanklich identifiziert werden. Wir schreiben den beiden konkurrierenden strukturellen Phasen daher folgende Charakteristika zu:

- Die metallische Phase lässt im Bild des Doppelaustausches geringe Bindungswinkel erwarten, was einer pseudokubischen Struktur gleichkommt. Dieser Fall ist inkompatibel mit einer statischen Jahn-Teller-Verzerrung der einzelnen $\mathrm{MnO}_{6}$-Oktaeder. Es ist daher von einem polaronischen Transport mit dynamischer Jahn-Teller-Verzerrung auszugehen. Es ist keine Orbital- oder Ladungsordnung vorhanden.

- Die isolierende Phase kennzeichnet sich durch statische Jahn-Teller-Elongationen der $\mathrm{MnO}_{6}$-Oktaeder, welche einen polaronischen Transport unterbinden. Die Bindungswinkel sind stark vergrößert und raumfüllende periodische Anordnungen entlang verschiedener Achsen verzerrter Oktaeder können komplexe Ladungsordnungen hervorrufen. Aufgrund der durch den Jahn-Teller-Effekt aufgehobenen Entartung stellt sich zudem möglicherweise eine orbitale Ordnung ein. Diese Ordnungsvorgänge können durch die Anwesenheit von Grenz- und Oberflächen wesentlich beeinflusst werden.

\section{Oberflächeneinflüsse}

Während im Volumen die Phasenanteile zum Beispiel thermisch oder durch Anlegen eines Magnetfeldes variiert werden können, muss an der Oberfläche nicht notwendigerweise die gleiche strukturelle Phasenverteilung vorliegen. Es ist stattdessen zu beachten, dass insbesondere die antiferromagnetisch ladungsgeordnete CE-Phase - ein Band-Isolator - nach theoretischen Erwägungen selbst bei optimal dotierten Manganaten in der Nähe von Grenzflächen besondere Stabilität erlangt. Der Doppelaustausch wird an der Oberfläche durch Ladungsverarmung - etwa wegen einer effektiven Überdotierung durch ein Sauerstoffdefizit - stark geschwächt. ${ }^{[74]}$

Die Bildung einer antiferromagnetischen Phase wird daher an der Oberfläche entscheidend begünstigt und sie könnte sogar den Grundzustand der Manganatoberfläche darstellen. Wie in Abschnitt 1.6 bereits dargestellt, wird diese Vermutung durch Beobachtungen magnetischer und auch elektrischer Dead Layers erhärtet. Für das Modell des Schaltmechanismus ist dieser Aspekt von besonderer Wichtigkeit, um auch in für das Volumen metallischen Regimen einen hochisolierenden Ausgangszustand zu verstehen. Andernfalls wäre für LSMO und bei tiefen Temperaturen auch für LCMO das beobachtete Vorliegen einer isolierenden Oberfläche in Anbetracht der metallischen Volumenphase nicht plausibel.

Tatsächlich gelangten Tsui et al. in ihren Untersuchungen des Schaltens an $\mathrm{Pr}_{0,7} \mathrm{Ca}_{0,3} \mathrm{MnO}_{3}$ mittels Impedanzspektroskopie zu dem Schluss, dass sich der Schalteffekt nur auf einen Dickenbereich von maximal $10 \mathrm{~nm}$ an der Oberfläche beschränkt. ${ }^{\text {97] }}$ Dementgegen stehen allerdings Ergebnisse von Jooss et al., die, auch für $\operatorname{Pr}_{0,7} \mathrm{Ca}_{0,3} \mathrm{MnO}_{3}$, sich tiefer in das Volumen erstreckende, strukturelle Änderungen während des Schaltens zeigen. ${ }^{[56]}$ Wir gehen, zumindest für die mit Strontium und Calcium dotierten Systeme, hier ebenfalls von einem oberflächennahen Schalten mit einem CE-artigen und bezüglich Dotierung und Temperatur robusten Grundzustand aus. Die genaue Natur des isolierenden Oberflächenzustandes ist zwar nur selten bekannt, die CE-Phase präsentiert sich jedoch für Manganate als eine sehr stabile, ladungsgeordnet-isolierende Konfiguration, welche bevorzugt an Grenzflächen nukleiert. ${ }^{[76]}$ 


\subsubsection{Modellbildung}

Die bisher gezeigten experimentellen Befunde sind deutliche Indizien dafür, dass zumindest eine strukturelle Komponente als wesentlicher Bestandteil im Schaltprozess involviert ist; in den nachfolgenden Kapiteln besprochene Resultate werden diese Vermutung noch weiter erhärten. In diesem Abschnitt wird nun ein auf den obigen Vorüberlegungen basierendes, strukturelles Modell vorgestellt. Die Zielsetzung besteht dabei nicht in einer vollständigen mikroskopischen Beschreibung des Schaltmechanismus - dies ist auf Grundlage der gegenwärtigen experimentellen Ergebnisse noch nicht möglich. Es soll vielmehr ein mögliches Szenario erarbeitet werden, welches verständlich macht, wie ein elektrisches Feld einen strukturellen Übergang und damit eine Widerstandsänderung bewirken könnte.

Wir beginnen mit einer Betrachtung der orbitalen Ordnung des isolierenden Zustands: Die CE-Phase ist ein Band-Isolator, welcher - wie in Kapitel 1 beschrieben - aus JahnTeller-elongierten $\mathrm{MnO}_{6}$-Oktaedern besteht, die sich innerhalb einer Ebene zu ZickzackKetten formieren; die Verbindung erfolgt über unverzerrte $\mathrm{Mn}^{4+}$-Plätze. Im Volumen sind Gitterverzerrungen wie Jahn-Teller-Elongationen der $\mathrm{MnO}_{6}$-Oktaeder in $x$-, $y$ - und $z$-Richtung energetisch gleichwertig. Daher ist ihre Orientierung, sofern nicht zusätzliche Einflüsse wie beispielsweise substratbedingte Anisotropien berücksichtigt werden müssen, willkürlich. Hypothetische CE-Cluster wie etwa im Idealfall der Hochtemperaturphase eines dreidimensionalen Einkristalls würden zu gleichen Teilen entlang aller drei kartesischen Achsen ausgerichtet sein. An der Oberfläche ist hingegen ein Symmetriebruch festzumachen, welcher diese Gleichgewichtung stört.

Die lagenweise Ausrichtung der Ketten nimmt unter dem Einfluss der symmetriebrechenden Oberfläche also eine Vorzugsorientierung ein. Demzufolge sind vermutlich alle verzerrten Oktaeder in der Ebene gestreckt und es besteht eine orbitale Ordnung aus $d_{3 x^{2}-r^{2}}$ und $d_{3 y^{2}-r^{2}}$-Zuständen auf den $\mathrm{Mn}^{3+}$-Plätzen. Die Ladungsdichteverteilung dieser Manganorbitale stellt ein elektrisches Quadrupolmoment dar. Es wechselwirkt daher prinzipiell mit einem inhomogenen elektrischen Feld $E$ beziehungsweise, genauer, dessen Gradienten. Ein Feldgradient ergibt sich zwangsläufig am Übergang zwischen zwei Phasen verschiedener Leitfähigkeit und ist, wenn wir die genauen, sich während des Phasenwachstums verändernden, geometrischen Verhältnisse vernachlässigen, ungefähr proportional zur angelegten Spannung.

Diese Situation ist in Abb. 5.4 veranschaulicht: Mit zunehmender Spannung an der AFM-Spitze ergibt sich für das in der Ebene liegende Quadrupolmoment eine immer größere elektrostatische Energie. Das andere Orbital ist hingegen günstiger orientiert, sodass sich die Energieaufspaltung zwischen den beiden Zuständen für größere Feldgradienten verkleinert. Es wäre also denkbar, durch Anlegen einer hinreichend großen Spannung die energetische Entartung der beiden Jahn-Teller-aufgespaltenen $e_{g}$-Zustände wiederherzustellen. Elektronen, welche nun der positiven Spannung folgend aus dem Volumen zur Oberfläche fließen, würden dann beide $e_{g}$-Zustände mit gleicher Wahrscheinlichkeit besetzen. Das Überkommen der Jahn-Teller-Barriere entspräche dem Übergang zur pseudokubischen Struktur, welche durch geringe Bindungswinkel eine große Leitfähigkeit ermöglicht. 
(a) HRS (Jahn-Teller-verzerrt)

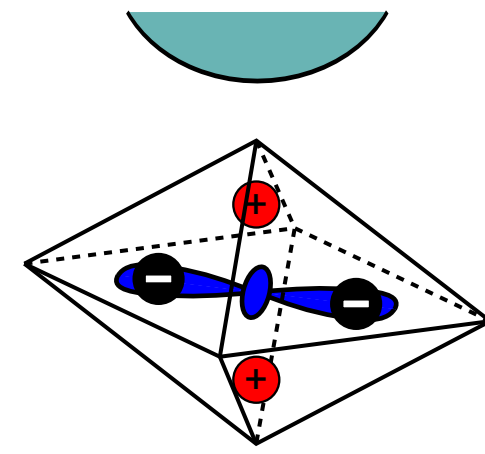

(b) LRS (pseudokubisch)

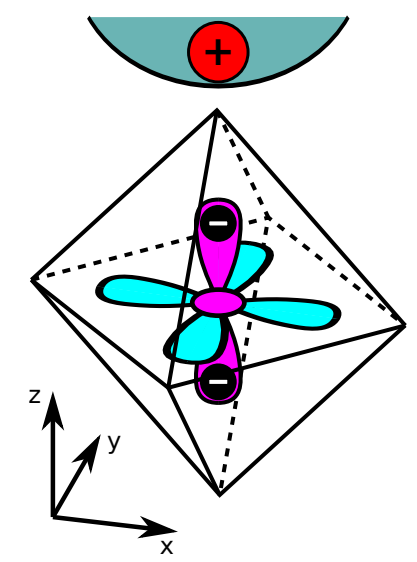

$f 3 z^{2}-r^{2} \quad f x^{2}-y^{2} \quad \infty \begin{gathered}3 x^{2}-r^{2} \\ 3 y^{2}-r^{2}\end{gathered}$

Abbildung 5.4: Modell eines strukturellen Übergangs. Die parallel zur Oberfläche orientierten $d_{3 x^{2}-r^{2}}$ und $d_{3 y^{2}-r^{2}}$-Orbitale stellen ein elektrisches Quadrupolmoment dar (a). Durch eine Spannung an der Kontaktelektrode hier der AFM-Spitze - kann eine Entartung der $e_{g}$-Orbitale wiederhergestellt und der Übergang zu einer höhersymmetrischen Struktur erzwungen werden (b).
Der angenommene ladungsgeordnet-isolierende Grundzustand der Manganatoberfläche könnte also durch Anlegen einer Spannung aufgrund eines starken lokalen Feldgradienten an der Oberfläche gestört werden. Das Ausbreiten der metallischen Region bei anliegender Spannung lässt sich ähnlich fassen: An der Phasengrenzfläche herrscht wieder ein großer Feldgradient, welcher dort die Ladungsordnung des Isolators zerstört (siehe Abb. 5.5).

Um darüberhinaus remanentes Schalten erklären zu können, muss außerdem der Frage nachgegangen werden, weshalb die Oberfläche nicht wieder in ihren isolierenden Grundzustand zurückfällt; die metallische Phase muss sicherlich als bestenfalls metastabil angesehen werden. Bei der Koexistenz verschiedener struktureller Phasen entstehen jedoch durch die gegenseitige Fehlanpassung elastische Verspannungen im Bereich der Domänengrenzen. Diese sind energetisch ungünstig und die entsprechende Grenzflächenenergie muss mit einer Volumenenergie verglichen werden, welche aus der mechanischen Stabilität der gesamten Domäne resultiert.

Es besteht dabei eine Analogie zum Beispiel zu den wohlbekannten ferromagnetischen Domänen. Auch hier lässt sich eine kritische Domänengröße festmachen, welche aus dem Verhältnis der stabilisierenden Volumenenergie innerhalb der Domäne und dem energetischen Verlust an ihrer Grenzfläche resultiert. Ein starker Einfluss muss sicherlich auch Defekten zugeschrieben werden, welche als Haftzentren Domänenwände lokal begünstigen können. Die ungeordnete Natur der Manganate vermag sich ferner vielleicht auf die Konkurrenz der beiden Phasen auszuwirken: Das energetische Gleichgewicht mag lokal besser austariert sein, sodass beide Phasen nebeneinander vorliegen können, oder anderenorts so sehr verletzt sein, dass die metallische Phase nicht stabil ist und nach Abschalten des Feldes nicht überlebt. In diesem Kontext lässt sich also auch die beobachtete Rückbildung metallischer Regionen begreifen dieser Aspekt wird in Abschnitt 5.4.2 ausführlich besprochen. Defekte - und damit auch insbesondere die Oberflächenmorphologie - könnten jedoch als Haftzentren eine selbstständige Rückkehr zum isolierenden Zustand verhindern. Durch ein derartiges 
pinning beziehungsweise feldgetriebenes depinning ließe sich auch die manchmal zu beobachtende starke Korrelation zwischen der Ausbreitung leitfähiger Regionen und der Morphologie verstehen (siehe Abb. 4.8).

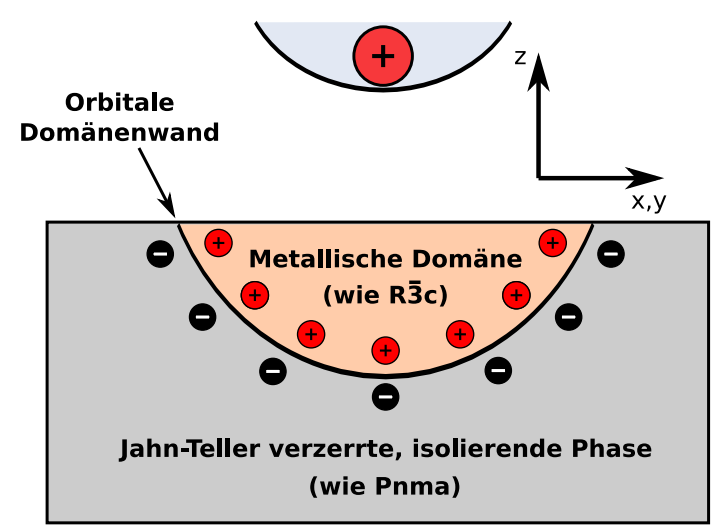

Abbildung 5.5: Ausbreitung der metallischen Region durch den elektrischen Feldgradienten an der Domänenwand.

\subsubsection{Offene Fragen}

Das aufgestellte Modell wird den wesentlichen Charakteristika des Schalteffektes bereits gerecht. Es bleiben jedoch Beobachtungen, bei deren Beschreibung es noch zu kurz greift. Problematisch ist beispielsweise das Verständnis des Zurückschaltens vom LRS zum HRS unter negativer Spannung. Zwar könnte eine positive Spannung mit dem Quadrupolmoment an der Oberfläche in beschriebener Weise interagieren, es ergäbe sich jedoch dieselbe elektrostatische Zusatzenergie wie bei der entgegengesetzten Polarität. Auch eine negative Spannung müsste also ein Schalten zum LRS begünstigen. In allen durchgeführten Messungen ist jedoch das exakte Gegenteil beobachtet worden: Negative Spannungen können den LRS nicht generieren, sondern allenfalls eine Zerstörung desselben bewirken.

Sofern die Spannungsschwelle also tatsächlich einem Überkommen der Jahn-Teller-Barriere entspricht, muss man sich fragen, in welcher Weise diese beim Zurückschalten in den HRS eigentlich überwunden werden muss. Die metallische Phase wird ja gerade als nicht verzerrt angenommen und das Bild des Quadrupolmoments einzelner Orbitale greift an sich zu kurz. Dennoch befinden sich die konkurrierenden Phasen auch als ausgedehnte Objekte kooperativer $\mathrm{MnO}_{6}$-Oktaeder natürlich in einem lokalen Energieminimum. Eine strukturelle Umordnung ist auf jeden Fall mit einer Energiebarriere verbunden, die aber möglicherweise auch durch den Ladungszu- beziehungsweise Abfluss, und somit die Bildung oder Zerstörung eines Oberflächendipols, überwunden wird. Dies entspricht letztendlich einer feldgetriebenen Änderung der effektiven lokalen Dotierung. Es sei darauf hingewiesen, dass auch das sehr verbreitete Bild der stark verzerrten, ladungsgeordneten CE-Phase eigentlich nicht stimmt: Neuere Untersuchungen zeigen, dass diese Konfiguration auch ohne starke Verzerrungen und mit nur schwacher Ladungsordnung zwischen $\mathrm{Mn}^{3,5+\delta}$ - und $\mathrm{Mn}^{3,5-\delta}$-Plätzen zustande kommen kann. ${ }^{[26]}$ Das orbitale und strukturelle System ist in Manganaten gewissermaßen „weich“. Ähnlich wie man in der mikroskopischen chemischen 
Unordnung eine Ursache für langskalige Phasenseparation finden kann, ist vielleicht auch die energetische Barriere zwischen den konkurrierenden Phasen eine sehr komplexe Folge der mikroskopischen Jahn-Teller-Verzerrungen. Die selbstständige Phasenseparation von Manganaten kann gedanklich sogar in den materialphysikalischen Kontext einer spinodalen Entmischung eingeordnet werden, wobei den Endgliedern des Systems die sich elektronisch und strukturell unterscheidenden Phasen entsprechen. ${ }^{[21]}$

Die genaue Ankopplung des Feldes an das Ladungs- und Orbitalsystem kann hier nicht ergründet werden. Über die Details der Orbitalordnung ist zu wenig bekannt, um wirklich von dem Quadrupolbild ausgehen zu können. Hinzu kommen die erwähnten Unzulänglichkeiten bei der genauen Erklärung des Zurückschaltens. Prinzipiell eignet sich hier auch die Annahme eines durch das Feld beeinflussten Oberflächendipolmomentes: Die feldinduzierte Ladungsverschiebung und somit Dotierungsänderung muss nur eine durch die starken Elektron-Phonon-Wechselwirkungen vermittelte, aus der strukturellen Phasenkonkurrenz resultierende Energiebarriere spüren. Das stark vereinfachende Modell macht nur plausibel, wie elektrische Felder eine Strukturänderung bewirken könnten.

Dass die lokalen Ladungsträgerdichten und elektronische Korrelationseffekte einen starken Einfluss auf die strukturellen Eigenschaften des Ionengitters ausüben, zeigen die bereits angesprochenen theoretischen Arbeiten an verwandten hochkorrelierten Perowskiten wie $\mathrm{KCuF}_{3}$ und LMO durch Leonov et al. ${ }^{[60,61]}$ Die rasanten Entwicklungen in der theoretischen Beschreibung hochkorrelierter Systeme, insbesondere auf Basis der dynamischen Molekularfeldtheorie, lassen auf eine Aufschlüsselung der Kopplungsmechanismen zwischen elektrischen Feldern und strukturellen Eigenschaften hoffen.

Vor der Ausarbeitung eines detaillierten, auf der Annahme eines strukturellen Überganges basierenden Modells sollte die prinzipielle Richtigkeit dieser Hypothese im Vergleich zu den in der Literatur etablierten Modellen eingehend geprüft werden. Dafür muss im Folgenden vor allem eine Beschreibung der Wachstumsdynamik der Domänen erarbeitet werden. Darüberhinaus muss die Diskrepanz zwischen dem hochisolierenden Ausgangszustand und dem HRS erklärt werden und eine Einordnung der Oberflächenmodifikationen während der Spannungspulse erfolgen. Schließlich gilt es, die beobachteten Einflüsse undotierter Decklagen auf das Schaltverhalten und das über diskrete Werte verlaufende, topographiekorrelierte Schalten phänomenologisch in das Szenario einer strukturellen Änderung einzugliedern. 


\subsection{Vergleich mit anderen Modellen}

In diesem Unterkapitel wird auf die in der Literatur diskutierten Erklärungsansätze der Modifikation einer Schottky-Barriere durch Ladungsakkumulation und der Elektromigration von Sauerstoffvakanzen eingegangen. Das Bild eines SCLC-Verhaltens mit einer durch die Erzeugung von Kristalldefekten veränderlichen Dichte an Ladungsträgerfallen von Tsui et al. ${ }^{[97]}$ setzt explizit eine Elektromigration durch starke Felder voraus und muss daher nicht gesondert diskutiert werden. Gleiches gilt für das Bild des Mott-Überganges nach Fors et al. ${ }^{[95]}$, welches ohnehin für Manganate von untergeordneter Bedeutung ist.

\subsubsection{Schottky-Barriere}

Das von Sawa et al. vorgeschlagene Modell einer Art Schottky-Barriere, ${ }^{[101]}$ deren Breite sich durch Ladungsakkumulation beziehungsweise -abfluss kontrollieren lässt, erweist sich im vorliegenden Fall als problematisch. So ist eine Übertragung auf die C-AFM-Ergebnisse schon konzeptionell schwierig, weil die Cantilever-Spitze eine bestimmte Oberflächenregion ja nur zeitweilig kontaktiert, ein Schottky-Kontakt also geschlossen und wieder gelöst würde. Demhingegen verbleibt die Leitfähigkeitsänderung jedoch auch in Abwesenheit der Spitze. Diese Persistenz beider Widerstandszustände auch ohne Existenz eines Manganat-MetallKontaktes deutet darauf hin, dass das Konzept einer Schottky-Barriere - auch, wie von Sawa et al. ${ }^{[101]}$ erwogen, eventuell durch elektrochemische Prozesse erweitert - nicht auf die hier behandelten Ergebnisse anwendbar ist. Es scheint vielmehr das Vorliegen einer Manganatgrenzfläche an sich ausreichend für das Widerstandsschalten zu sein, gleichgültig ob zu einer Elektrode oder auch nur zum Vakuum.

Die mikroskopischen Prozesse mögen denen einer Schottky-Barriere dennoch ähneln: Auch das obige, strukturelle Modell geht ja beispielsweise von einer Verarmungszone dem sauerstoffdefizitären, effektiv überdotierten Bereich an der Oberfläche aus. Auch die von Sawa et al. ${ }^{[101]}$ vorgeschlagene Kontrolle der Leitfähigkeit durch eine Besetzung oder Entvölkerung von Grenz- beziehungsweise Oberflächenzuständen könnte mit der Dotierungsänderung während des strukturellen Übergangs identifiziert werden.

Die in Abb. 4.5 gezeigten Kennlinien zeigen jedoch allenfalls geringfügige Asymmetrien, keineswegs aber wirklich gleichrichtendes Verhalten, wie es von einem einzelnen SchottkyKontakt zu erwarten wäre. Die asymmetrischen Kennlinien ließen sich ferner schon erklären, wenn man zwischen Cantileverspitze und Probe einen asymmetrischen Tunnelkontakt annimmt: Die verschiedenen Austrittsarbeiten des Manganates und der Cantileverbeschichtung ließen eine asymmetrische Kennlinienform nach dem Brinkman-Modell ${ }^{[36]}$ erwarten, welche qualitativ sehr gut mit den experimentellen Ergebnissen übereinstimmt. ${ }^{\mathrm{e}}$

Demnach sollte die Ausbildung einer Schottky-Barriere hier also allenfalls von untergeordneter Bedeutung sein und nicht als zentrales Element des Schaltmechanismus angesehen werden. Realistischer erscheinen stattdessen Modelle, wie von Jooss et al. vorgestellt, welche die Kopplung einer sich an der Elektrode ausbildenden Schottky-Barriere mit der strukturel-

\footnotetext{
${ }^{\mathrm{e}}$ Die Fit-Parameter ergaben für die Brinkman-Formel unphysikalische Werte, weshalb auf eine detailliertere Analyse verzichtet wurde. Wahrscheinlich ist die Kontaktbeschaffenheit komplexerer Natur; hinzu kommt eine mögliche Verfälschung durch die Spannungsabhängigkeit des dominierenden Widerstandsbeitrages von der Manganatoberfläche.
} 
len Konfiguration des Materials enthalten. ${ }^{[106]}$ Auch hier wird deutlich, dass der tatsächliche Mechanismus möglicherweise nicht auf eines der Bilder zu reduzieren ist, sondern in derart hochkorrelierten Materialien möglicherweise nur eine Kombination elektronischer und struktureller Erklärungsansätze zielführend ist. Das in dieser Arbeit vorgestellte strukturelle Bild ähnelt denen von Sawa et al. ${ }^{[101]}$ und Jooss et al. ${ }^{[106]}$ insofern, dass eine elektronische beziehungsweise polaronische Ladungsverschiebung die Widerstandsänderung bewirkt und eben nicht ein elektrochemischer Effekt.

\subsubsection{Sauerstoffleerstellenmigration}

Unter den Perowskiten gibt es vor allem für Strontiumtitanat Indizien dafür, dass die Elektromigration von Sauerstoffleerstellen als ein wesentlicher Bestandteil in den Schaltmechanismus involviert ist. ${ }^{[142]}$ Zwar liegen bis heute keine eindeutigen Hinweise vor, aktuelle Arbeiten von Muenstermann et al. zeigen aber unter anderem eine mögliche Korrelation zwischen den nanoskaligen Schalteigenschaften und Kristalldefekten. ${ }^{[141]}$ Dies ist insofern bemerkenswert, als an Versetzungen eine erhöhte Beweglichkeit von Sauerstoffionen denkbar ist (,pipelike diffusion").

Das Modell eines auf der Elektromigration von Sauerstoffleerstellen fußenden Widerstandsschaltens findet gegenwärtig breite Zustimmung und wird auch für Manganate in Betracht gezogen. ${ }^{[145]}$ Es ist nicht nur bestechend intuitiv, sondern erklärt auch im hier vorliegenden Falle die beobachteten Polaritäten - eine Elektrode auf positivem Potential wirkt auf Sauerstoffionen attraktiv, sodass die Leerstellenkonzentration an der Grenzfläche reduziert und die Leitfähigkeit verbessert wird. In Übereinstimmung mit dieser Vorstellung ist auch für Manganate ein erheblicher Einfluss der Sauerstoffleerstellenkonzentration auf den elektrischen Widerstand bekannt; ${ }^{[98]}$ auch weisen Perowskitoberflächen tatsächlich oftmals deutliche Sauerstoffdefizite auf. Dennoch werfen insbesondere im Falle der Manganate einige Befunde Zweifel an diesem ionischen Szenario auf.

Im Folgenden werden diese Aspekte aufgelistet; die Einwände sind dabei größtenteils allgemeiner Natur.

- Die Fähigkeit zur Elektromigration ist direkt mit der thermisch aktivierten Eigendiffusion der Sauerstoffleerstellen verknüpft. Daher sollte eine starke Temperaturabhängigkeit bestehen und die Schalteffekte müssten für niedrige Temperaturen sukzessive unterdrückt werden. Sie wurden jedoch nicht nur hier bei Raumtemperatur sowie für $T=100 \mathrm{~K}$ aufgefunden, sondern an nanokristallinen LSMO-Proben sogar für $T=5 \mathrm{~K} \cdot{ }^{[107]}$ Ein auf Elektromigration basierendes Szenario muss also entweder eine lokale Erwärmung während des Schaltprozesses annehmen oder einen zusätzlichen, beispielsweise durch große Stromdichten verursachten, diffusionsfördernden Mechanismus enthalten.

- Über die Diffusionskoeffizienten für Sauerstoffionen in perowskitischen Manganaten, welche meist an gesinterten Proben bestimmt wurden, finden sich verschiedene Angaben. ${ }^{[146,147]}$ Für dünne epitaktische Manganatfilme sind sie, wie durch Malavasi \& Flor an $\mathrm{La}_{0,85} \mathrm{Na}_{0,15} \mathrm{MnO}_{3}$ bestimmt, vergleichsweise klein. ${ }^{[148]}$ 
- Die Untersuchungen von Malavasi \& Flor ${ }^{[148]}$ ergaben für den Sauerstoffionentransport in epitaktischen $\mathrm{La}_{0,85} \mathrm{Na}_{0,15} \mathrm{MnO}_{3}$-Filmen Aktivierungsenergien von mindestens der gleichen Größe wie die Jahn-Teller-Energie $E_{\mathrm{JT}}$. Zur Fortbewegung von Sauerstoffionen durch den Kristall ist also mindestens eine Energie des ungefähr gleichen Betrages erforderlich, wie um eine Jahn-Teller-Verzerrung aufzuheben.

- Es gibt im Rahmen des Sauerstoffmigrationsszenarios kein einfaches Bild, in welchem die Existenz einer scharfen Spannungsschwelle verständlich ist. Elektromigration allein sollte sich mit ansteigender Spannung vor allem in höheren Schaltgeschwindigkeiten bemerkbar machen, nicht aber unterhalb eines kritischen Wertes vollständig unterbunden werden.

- Im Bilde der Sauerstoffmigration muss das Innere des Filmes als Reservoir dienen, von wo aus der feldgetriebene Ionentransport zur defizitären Oberfläche erfolgt. Die Eindiffusion von Ionen aus der Atmosphäre - für die dieser Abhandlung zugrundeliegenden Experimente schon wegen der Vakuumbedingungen praktisch undenkbar - oder aus eventuellen Kontaminationen an der Oberfläche würde entgegen der Feldrichtung eher erschwert werden.

- Bei den beobachteten „Satellitenflecken“ stünde die Transportrichtung - vom Volumen zur Oberfläche - senkrecht zum Stromfluss und auch zu der elektrischen Feldrichtung in der Filmebene. Daher ist nicht ersichtlich, weshalb sich eine lokale Sauerstoffanreicherung fernab der Cantileverspitze ereignen sollte. Im Rahmen des strukturellen Szenarios könnten Inhomogenitäten jedoch eine örtlich reduzierte Schwellspannung ergeben, etwa durch lokale Verspannungen und Defekte. Zudem kann an Defekten entlang des Strompfades auch eine größere lokale Feldstärke herrschen, wodurch trotz des vergleichsweise großen Abstandes zur Cantileverspitze ein Schalten initiiert werden könnte.

- Es wurden zwar gewisse Korrelationen mit der Topographie beobachtet, die hohe epitaktische Qualität der untersuchten Manganate spricht jedoch gegen einen nur an Defekten möglichen Schaltvorgang. Es war zudem oftmals möglich, Probenbereiche flächendeckend in den metallischen Zustand zu schalten - ganz im Gegensatz zu der vor allem punktuellen Schaltbarkeit von Strontiumtitanat. ${ }^{[94]}$

- Diffusion kann zwar auch entlang der Oberfläche um Größenordnungen schneller ablaufen als im Volumen. Oberflächenmigration würde allerdings umliegende Bereiche zusätzlich an Sauerstoff verarmen lassen. Dies sollte bei der Bildung metallischer Flecken durch einen Spannungspuls zu einer Zerstörung benachbarter metallischer Inseln führen. Die Möglichkeit des großflächigen Umschaltens widerspricht also auch dieser Annahme.

Der größte Schwachpunkt des Migrationsmodells bei einer Übertragung auf Manganatsysteme ist also die vermutlich zu geringe Sauerstoffmobilität. Nian et al. sehen hier in großen Stromdichten eine unterstützende Funktion. ${ }^{[145]}$ Im nächsten Abschnitt wird dieser Aspekt detailliert untersucht; zusätzlich werden die dabei auftretenden Heizeffekte abgeschätzt. Weitere Argumente, welche eine Entscheidung über die Anwendbarkeit des ionischen Szenarios 
im vorliegenden Falle erleichtern, werden in den darauffolgenden Abschnitten - insbesondere in Bezug auf die Domänendynamik - erarbeitet.

\subsection{Stromdichten und Heizeffekte}

Wie im vorigen Unterkapitel dargestellt, erscheint das elektrochemische Szenario im Falle der Manganate nur dann sinnvoll, wenn zum einen ein diffusionsfördernder Mechanismus und andererseits eine plausible Begründung für die Existenz der Schwellspannung gefunden werden kann. In diesem Abschnitt untersuchen wir die während des Schaltens herrschenden Stromdichten und die daraus resultierenden Heizeffekte. Eine lokale Erwärmung der Probe in den schaltenden Bereichen könnte beispielsweise eine größere Mobilität der Sauerstoffvakanzen erklären.

Die Stromdichte kann aber auch selbst als der treibende Faktor angesehen werden: Stoßprozesse mit Elektronen könnten aktivierte Ionen beschleunigen, wie beim „Elektronenwind“ in metallischen Leitern. Aufgrund der im Vergleich zur Ionenmasse sehr kleinen Elektronenmasse ist der Impulsübertrag bei einem einzelnen Stoßprozess verschwindend; für einen signifikanten Effekt sind daher große Stromdichten erforderlich.

Nian et al. äußerten die Vermutung eines komplexeren Schaltprozesses auf Basis einer stromdichtegetriebenen Elektromigration und entwarfen ein entsprechendes Bild zur Erklärung der beobachteten Spannungsschwelle: ${ }^{[145]}$ Es gibt für $\mathrm{YBa}_{2} \mathrm{Cu}_{3} \mathrm{O}_{7-\delta}$ Indizien dafür, dass durch einen Franck-Condon-artigen Mechanismus die Sauerstoffbindungen geschwächt werden. ${ }^{[149]}$ Bei großen Stromdichten würden also viele ,antibindende“ elektronische Zustände besetzt werden, und die Verankerung des Sauerstoffs im Kristallgitter geschwächt. Die Schwellspannung wäre die zur Besetzung dieser höherenergetischen Zustände zu überkommende Barriere.

Im Folgenden werden die typischen Größen der in den einzelnen Widerstandszuständen kurz vor dem Schaltprozess, als bei Spannungen knapp unterhalb von $U_{\mathrm{C}}$, auftretenden Stromdichten und Heizeffekte abgeschätzt. Davon ausgehend werden im Anschluss die unter 4.5.2 dargestellten Oberflächenschädigungen diskutiert.

\subsubsection{Ausgangszustand vor dem erstmaligen Schalten}

Eine Abschätzung der erreichten Stromdichten während der C-AFM-Schaltexperimente gestattet der in Abschnitt 3.5 bestimmte Mindestkontaktradius von $a=1,1 \mathrm{~nm}$. Da die Ströme im Ausgangszustand der Probe selbst bei anliegenden Spannungen von mehreren Volt - aber noch unterhalb der Schwellspannung $U_{\mathrm{C}}$ - häufig nicht mehr als $I=10 \mathrm{pA}$ betragen, liegen die maximalen Stromdichten hier in der Größenordnung $10^{2} \mathrm{~A} / \mathrm{cm}^{2}$.

Diese eigentlich eher moderaten Werte wurden von Nian et al. ebenfalls beobachtet und ihnen im Rahmen des obigen Bildes eine mögliche kritische Rolle bei der Aktivierung der Sauerstoffmobilität zugesprochen. ${ }^{[145]}$ Es ist dabei aber zu beachten, dass diese Stromdichten im Falle der hier vorliegenden C-AFM-Ergebnisse nur einen Maximalwert angeben - wahrscheinlich ist die reale Kontaktfläche deutlich größer, die Stromdichten also noch geringer. Mit Hilfe des abgeschätzten Kontaktradius und des Stromes kann ferner die lokale Joulesche Erwärmung abgeschätzt werden, welche die Frage nach der Möglichkeit einer thermischen Aktivierung des Ionentransportes beantwortet. 
Aus einem einfachen thermischen Modell, welches auf der makroskopischen Wärmeleitungsgleichung basiert, ergibt sich für die Temperaturerhöhung folgende Formel:[150,151]

$$
\Delta T=\frac{U I}{2 \pi k_{\mathrm{th}} a} .
$$

Dabei ist $k_{\text {th }}$ die thermische Leitfähigkeit der Probe; wir verwenden hier den von Weber et al. ${ }^{[152]}$ an $\mathrm{La}_{0,7} \mathrm{Sr}_{0,3} \mathrm{MnO}_{3}$ bestimmten Wert von $k_{\mathrm{th}} \approx 4,7 \mathrm{Wm}^{-1} \mathrm{~K}^{-1}$. $^{\mathrm{f}}$ Es ergeben sich so für den hochisolierenden jungfräulichen Oberflächenzustand mit $I=10 \mathrm{pA}$ nur sehr kleine Temperaturerhöhungen von $\Delta T \leq 0,01 \mathrm{~K}$. Im hochisolierenden Ausgangszustand reichen also vermutlich weder die Stromdichten, auf keinen Fall aber die daraus entstehenden Heizeffekte, aus, um Diffusionseffekte derart zu fördern, dass das erstmalige Schalten in den LRS erklärlich wäre.

\subsubsection{Metallischer Zustand (LRS)}

Wir untersuchen jetzt in gleicher Weise das Schalten vom metallischen LRS zurück zum hochohmigen HRS. Während für kleinere Spannungen noch ein Widerstand von $R_{\mathrm{LRS}} \approx 12 \mathrm{M} \Omega$ (vergleiche Abb. 4.5) ermittelt wurde, so findet danach ein rascher Anstieg der $I-U$ Kennlinie statt. Diese erreichte schon für wenige hundert Millivolt den apparativ maximal messbaren Strom von $50 \mathrm{nA}$. Nahe der Schwellspannung $U_{\mathrm{C}}$ ergäbe sich so unter linearer Extrapolation des steilen Anstiegs schon ein Strom von bis zu einigen Mikroampere. Zusätzlich ist eine Abnahme des elektrischen Widerstandes direkt durch die größeren Spannungen (zum Beispiel durch Tunnelbeiträge am Kontakt) und indirekt über die einsetzende Erwärmung möglich. ${ }^{g}$ Spitzenwerte in der Größenordnung von zehn Mikroampere erscheinen somit ohne weiteres denkbar. Die entsprechenden Stromdichten nehmen demnach sehr große Werte von bis zu $10^{7} \ldots 10^{8} \mathrm{~A} / \mathrm{cm}^{2}$ an.

Angesichts dieser lokal sehr großen Stromdichten kann eine dadurch geförderte Elektromigration sicherlich nicht ausgeschlossen werden. Dass die Stromdichte jedoch als primärer Auslöser den Schaltprozess treibt, erscheint angesichts der grob eingehaltenen Spannungssymmetrie in den gemessenen Schaltzyklen nicht plausibel. Wären nämlich die im LRS möglichen großen Stromdichten zum Schalten erforderlich, so erschiene das erste Schalten vom Ausgangszustand zum LRS unerklärlich. Läge andererseits die kritische Stromdichte zur Förderung der Sauerstoffmigration tatsächlich schon in der Größenordnung von $10^{2} \mathrm{~A} / \mathrm{cm}^{2}$, also den im Ausgangszustand erreichbaren Werten, so müsste beim Zurückschalten zum HRS schon eine negative Spannung von weniger als einem Millivolt genügen, um durch solche Stromdichten an der Kontaktfläche ein Zurückschalten zu induzieren. Dies widerspricht jedoch dem beobachteten Verhalten von in der Größenordnung übereinstimmender positiver und negativer Schaltschwelle.

Einhergehend mit den vergrößerten Stromdichten gewinnt im LRS auch die lokale Aufheizung der Probe an Gewicht. Mit einer Schwellspannung $\left|U_{\mathrm{C}}\right| \approx 3 \mathrm{~V}$ und einem Strom von

\footnotetext{
${ }^{\mathrm{f}}$ Jegliche thermischen Verluste und die Temperaturabhängigkeit der Wärmeleitfähigkeit werden in dieser groben Abschätzung ignoriert.

${ }^{\mathrm{g}}$ Außerdem ist noch einmal darauf hinzuweisen, dass es sich bei Abb. 4.5 um gemittelte Kennlinien handelt; einzelne LRS-Kurven zeigten daher noch größere Leitfähigkeiten.
} 
$I=10 \mu \mathrm{A}$ ergibt sich, wieder gemäß dem oben verwendeten, makroskopischen thermischen Modell ${ }^{[150]}$, bespielsweise eine deutliche Temperaturerhöhung:

$$
\Delta T \approx 900 \mathrm{~K} \text {. }
$$

Dieser Heizeffekt ist so stark, dass hier - auch unabhängig von der Stromdichte - tatsächlich eine stark erhöhte Beweglichkeit der Sauerstoffionen anzunehmen ist. Weil aber die Stromdichten und Erwärmungen im erstmaligen Schaltprozess offensichtlich keine Rolle spielen können, erscheint es wiederum unnatürlich, hierin die Ursache für das Zurückschalten zu suchen. Es ist jedoch durchaus denkbar, dass als Folge eines Schaltens in den LRS durch die dann fließenden großen Ströme auch eine Änderung des Konzentrationsprofils der Sauerstoffionen entsteht, die sich zusätzlich auf die Transporteigenschaften auswirkt. Möglicherweise erklärt ein solcher Heizeffekt die häufige Diskrepanz zwischen dem Ausgangszustand und dem HRS. Positive Spannungen und die starke Erwärmung nach dem ersten Schaltprozess könnten einerseits eine Konzentrationsanpassung der sauerstoffdefizitären Oberflächenregion an das Volumen bewirkt haben, wodurch die Leitfähigkeit eine Verbesserung erfährt. Ebenso könnten solche drastischen Einflüsse vielleicht auch eine isolierende Oberflächenkontamination oder ein Dead Layer beseitigen.

Dass die großen Stromdichten und die extremen Heizeffekte jedoch nur als Wirkung und keinesfalls als Ursache des Schalteffektes verstanden werden dürfen, zeigt auch die beobachtete selbstständige Rückbildung metallischer Domänen. In diesem Fall fließt überhaupt kein Strom und die Eigendiffusion erscheint, wie zuvor ausgeführt, bei Raumtemperatur zu klein.

\subsubsection{Isolierender Zustand (HRS)}

Abschließend soll jetzt noch der HRS analysiert werden. Hier erscheinen bei $U=3 \mathrm{~V}$ anhand von Abb. 4.5 Ströme bis zu einigen zehn Nanoampere realistisch. Die entsprechenden Stromdichten können mit etwa $10^{5} \mathrm{~A} / \mathrm{cm}^{2}$ bereits deutlich größer als im Ausgangszustand sein, die lokale Erwärmung ist jedoch mit nur einigen Kelvin nicht relevant. Diese Ergebnisse untermauern die obigen Symmetrieerwägungen bezüglich der Schwellspannung. Selbst wenn man für verschiedene Polaritäten beziehungsweise Schaltrichtungen auch unterschiedliche kritische Stromdichten annähme, wären die Resultate so unerklärlich: Die Schaltrichtung und Schwellspannung unterscheiden sich im Ausgangszustand und im HRS - ganz im Gegensatz zu den herrschenden Stromdichten - nicht. Der gleiche Schaltprozess in den LRS kann also offensichtlich auch bei sehr unterschiedlichen Stromdichten ausgelöst werden.

\subsubsection{Morphologische Änderungen}

In Abschnitt 4.5.2 wurden die gelegentlich beobachtbaren morphologischen Veränderungen der Oberfläche als eine Schädigung des Materials bei großen Spannungen eingeordnet. Auch wurden häufig gleichzeitige Einbußen in der Spitzenqualität des C-AFM-Cantilevers festgestellt. Die Abschätzungen in den vorigen Abschnitten zeigten, dass sobald die Probe in den LRS geschaltet ist, tatsächlich sehr große Stromdichten und drastische Heizeffekte resultieren können. 
Als Folge so ausgeprägter lokaler Erwärmung ist eine massive thermische Schädigung des Manganates, eventuell bis hin zu einer Amorphisierung, plausibel. So ließen sich beispielsweise die isolierenden Zentren metallisch geschalteter Regionen erklären. Die Entstehung zum Teil ausgeprägter Erhebungen impliziert jedoch eher eine Ansammlung von Spitzenmaterial auf der Oberfläche. Die hohen Temperaturen und die Spitzenform geben Anlass zur Vermutung einer thermisch assistierten Feldverdampfung von Pt-Ionen aus der Beschichtung. Derartige Effekte sind in der verwandten Rastertunnelmikroskopie keine Seltenheit. ${ }^{[38]}$ Dass dort fast ausschließlich die Bewegung von Kationen als relevant angesehen wird, deckt sich ferner mit der Beobachtung einer Entstehung solcher Modifikationen ausschließlich für positive Spannungen. Für große negative Spannungen und Stromdichten - vor dem Zurückschalten in den HRS - wurde keine Hügelbildung festgestellt.

Das genaue Verständnis der Entstehung solcher Oberflächenmodifikationen liegt außerhalb der Zielsetzung dieser Arbeit. Die Auswahl vor allem solcher Ergebnisse, die ohne derartige, ungewünschte Oberflächenmodifikationen erzielt werden konnten, erscheint aber im Nachhinein gerechtfertigt. ${ }^{\text {h }}$

\footnotetext{
${ }^{\mathrm{h}}$ Es wurden erste Versuche unternommen, durch geeignete Vorwiderstände den übermäßigen Stromfluss zu verhindern. Hierbei wurden aber negative Auswirkungen auf die Remanenz des Schaltens festgestellt. Wünschenswert wäre in diesem Zusammenhang eine dynamische Abregelung der Spannung beim Schalten, um ein Überschreiten eines wählbaren Maximalstromes zu vermeiden. Diese ist jedoch werkseitig instrumentell nicht vorgesehen und nur schwer nachträglich integrierbar - die erste Vorverstärkung des Stromsignals erfolgt bereits im UHV.
} 


\subsection{Domänenkriechen}

Dieser Abschnitt ist der zeitlichen Entwicklung metallischer Domänen gewidmet. Dabei werden die Wachstumsdynamik während des Schaltpulses und die Rückbildungen, welche sich nach Pulsende ereignen können, behandelt. Die Zeitabhängigkeit der Domänengröße unter angelegter elektrischer Spannung erlaubt wichtige Aufschlüsse über das Wesen des Schaltprozesses; man kann sie, wie im Folgenden dargelegt, sogar als eine Art Kriterium verstehen, welches den ionischen von dem strukturellen Mechanismus unterscheidbar macht:

- Ginge man etwa von einem diffusiven Prozess aus, das heißt von einem Domänenradius proportional zur Diffusionsstrecke $s$ der Sauerstoffvakanzen, so erwartete man eine wurzelförmige Zeitabhängigkeit $s \propto \sqrt{t}$. Dieses Verhalten entspräche dabei allerdings bloß der rein konzentrationsgetriebenen, thermisch aktivierten Diffusion. In Anbetracht der starken lokalen elektrischen Felder, durch welche die Sauerstoffionen beziehungsweise -vakanzen die zur Erklärung des Widerstandsschaltens notwendige gerichtete Beschleunigung erfahren sollten, erscheint ein derartiges Bild unrealistisch.

- Elektromigration, das heißt durch ein statisches, äußeres elektrisches Feld beschleunigte Ladungsträger, wäre demnach plausibler. Hier würde die Ausbreitungsgeschwindigkeit anwachsen, bis sich ein Gleichgewicht zwischen dem beschleunigenden elektrischen Feld und abbremsenden Reibungskräften eingestellt hat. Danach findet also eine in der Zeit lineare Vergrößerung der Domänen statt.

- Das oben vorgestellte strukturelle Modell ließe ein anderes Wachstumsgesetz erwarten, welches die feldgetriebene Ausbreitung der strukturellen Änderung unter Konkurrenz elastischer Kräfte mit den Potentialen von Haftzentren beschreibt. Derartige „Kriechphänomene" sind in der Festkörperphysik weit verbreitet und beispielsweise von der Vergrößerung ferromagnetischer ${ }^{[153]}$ und ferroelektrischer ${ }^{[144]}$ Domänen in äußeren Feldern, dem mechanischen Kriechen in metallischen Gläsern ${ }^{[154]}$ und schließlich von der Dynamik von Flussschläuchen in Supraleitern 2. Art ${ }^{[55]}$ bekannt. Ihre theoretische Behandlung gestaltet sich sehr anspruchsvoll und hängt unter anderem von der Dimensionalität der Domänengrenze und von der Form der Haftpotentiale ab, welche die Domänenwandbewegung hemmen.

Es wird zuerst, um die in Abb. 4.12 gezeigte Spannungs- und Pulsdauerabhängigkeit $\mathrm{zu}$ verstehen, eine Analyse im Kontext der Kriechtheorie unternommen, dann wird die zeitaufgelöste Beobachtung eines Erholungsprozesses (vergleiche Abschnitt 4.6.1), das heißt das sukzessive Verschwinden einer metallischen Oberflächenregion, diskutiert. Zum Abschluss werden die unter 4.6.2 aufgeführten Versuche mit verschiedenen Sequenzen von Spannungspulsen, welche eine Art kombiniertes Kriecherholungsverhalten zeigen, besprochen.

\subsubsection{Analyse des Domänenwachstums in Abhängigkeit von den Pulsparametern}

In diesem Abschnitt wollen wir die Entwicklung des elektrischen Feldgradienten in Abhängigkeit vom Domänenradius modellartig untersuchen, um ihn dann als treibende Kraft in einer 
Kriechformel zu verwenden. Dies ermöglicht, die Messergebnisse des Pulsrasterexperimentes aus Abschnitt 4.5.3 aufzugreifen und zu entscheiden, inwiefern die beobachtete zeitliche Entwicklung der Domänengröße in Abhängigkeit von der angelegten Spannung mit der Vorstellung eines strukturellen Kriechens, also dem vorgestellten Modell, vereinbar ist. Die bereits zuvor erwähnte, phänomenologische Ähnlichkeit mit den C-AFM-Studien von Paruch et al. ${ }^{[143]}$ und Tybell et al..$^{[144]}$ sei an dieser Stelle noch einmal betont. Darin wurde das Wachstum ferroelektrischer Domänen durch Spannungspulse erzwungen und anschließend ihre Größe mittels piezoelektrischer Kraftmikroskopie untersucht. Da dort mit einer verwandten Methodik ein Kriechverhalten nachgewiesen werden konnte, orientiert sich die im Folgenden durchgeführte Modellierung stark an diesen Arbeiten. Die Unterschiede ergeben sich aus den geometrischen Details und der Anpassung an das strukturelle Modell.

\section{Abschätzung der lokalen Feldgradienten am Domänenrand in Abhängigkeit von der Domänengröße}

Es soll zunächst die qualitative Abhängigkeit des elektrischen Feldgradienten an der Domänenwand, welchem im strukturellen Modell die Bedeutung der treibenden Kraft zukommt, in Abhängigkeit von ihrer Ausdehnung, also dem Abstand zur Cantileverspitze, bestimmt werden. Wir vereinfachen die geometrischen Verhältnisse dazu zunächst gemäß Abb. 5.6.

(a) Reale Kontaktverhältnisse (schematisch)

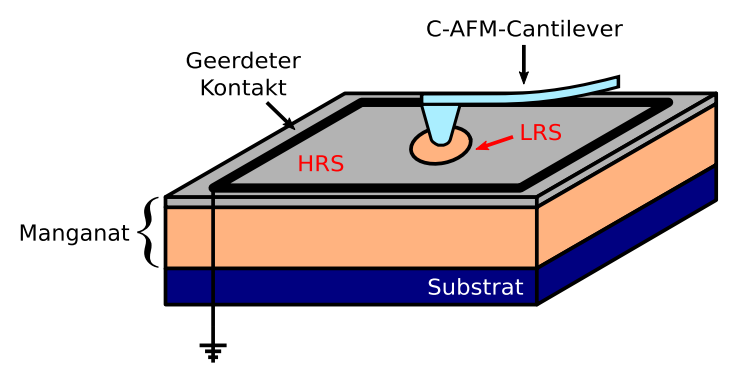

(b) Idealisierte Geometrie

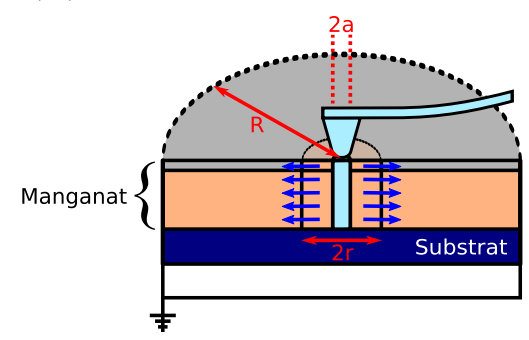

Abbildung 5.6: Modellierung der Feldgeometrie durch ein Zylinderschalenmodell. (a) zeigt schematisch die realen Kontaktverhältnisse: Am Rand der isolierenden Probenoberfläche liegt ein großer, viereckiger Klemmkontakt auf. Die idealisierte Geometrie zur Beschreibung des Feldverlaufs ist in (b) veranschaulicht: Die Außenelektrode und die Cantileverspitze werden als konzentrische Zylinderschalen verzerrt abgebildet; auch die Domänenwand wird als zylinderförmig angenommen.

Die äußere, geerdete Elektrode wird abweichend von den realen Bedingungen als die Probe zylinderförmig einfassend angenommen. Die Stromdichte auf der Mantelfläche einer ebenfalls näherungsweise als zylinderförmig behandelten Domänenwand ${ }^{i}$ beträgt:

$$
j=\frac{I}{2 \pi r d} .
$$

${ }^{\mathrm{i}}$ Diese Hypothese ist insbesondere für Flecken, deren Ausdehnung von mindestens ähnlicher Größe wie die Schichtdicke $d$ ist, wahrscheinlich gut erfüllt und sollte auch in unserem Falle noch als Näherung geeignet sein. 
Entsprechend gilt bei einer elektrischen Leitfähigkeit $\sigma$ für das $E$-Feld:

$$
E=\frac{I}{2 \pi \sigma r d} .
$$

Der Spannungsabfall über die gesamte Probe lässt sich aus der Integration des nach außen radial abfallenden Feldes zwischen den Elektroden gewinnen. In diesem Falle approximieren wir die durch den Cantilever gebildete Elektrode als mit den anderen Zylindern konzentrischen, metallischen „Schlauch“. Auch diese Approximation stellt eine grobe Vereinfachung dar, welche insbesondere für kleine, mit der Kontaktfläche vergleichbare Domänen versagt. Die betrachteten Flecken sind jedoch alle deutlich größer, $r \gg a$, und diese Annahme sollte daher eine hinreichend gute Näherung darstellen. Wir schreiben also:

$$
U=\int_{a}^{R} E \mathrm{~d} r=\frac{I}{2 \pi d \sigma} \ln \frac{R}{a} .
$$

Für den Strom erhält man entsprechend die folgende Beziehung:

$$
I=\frac{2 \pi d \sigma U}{\ln (R / a)}
$$

Durch Einsetzen in Gleichung 5.4 ergibt sich nun $E \propto \frac{U}{r}\left(\ln \frac{R}{a}\right)^{-1}$. In unserem Falle interessiert jedoch nur die Abhängigkeit des elektrischen Feldes von der Spannung $U$ und der Domänengröße $r$. Wir erhalten dafür folgende Proportionalität:

$$
E \propto \frac{U}{r}
$$

In dem obigen Schalenmodell schreiben wir jetzt der metallischen Domäne die homogene, große Leitfähigkeit $\sigma_{1}$ und der Umgebung einen kleineren Wert $\sigma_{2} \ll \sigma_{1}$ zu. $^{\mathrm{j}}$ Im Falle einer zwischen Außenelektrode und Cantileverspitze anliegenden Spannung entsteht nun an der Domänenwand eine Diskontinuität im elektrischen Feld. Beschreiben $E_{1}$ und $E_{2}$ die elektrische Feldstärke direkt innerhalb respektive außerhalb der Grenzfläche, so erwarten wir:

$$
\frac{E_{1}}{E_{2}}=\frac{\sigma_{2}}{\sigma_{1}} .
$$

Unter der Voraussetzung, dass die Breite der Domänenwand und der qualitative Verlauf der elektrischen Feldstärke in ihrer unmittelbaren Umgebung nicht wesentlich vom Domänenradius $r$ abhängen, können wir außerdem eine Proportionalität zwischen der Stufenhöhe dieser Diskontinuität und dem lokalen elektrischen Feldgradienten vermuten:

$$
\left.\nabla E\right|_{r} \quad \propto \quad E_{2}-E_{1} \quad=\left(1-\frac{\sigma_{2}}{\sigma_{1}}\right) E_{2} .
$$

\footnotetext{
${ }^{j}$ Grundsätzlich bedeutet der Leitfähigkeitssprung auch eine Änderung im Feldverlauf, welche im obigen geometrischen Modell berücksichtigt werden müsste. Da aber eine ausgeprägte Längenhierarchie $R \gg$ $r \gg a$ vorliegt, sollte bei der Modellierung des Feldverlaufs die Leitfähigkeitsänderung im Vergleich zu geometrischen Einflüssen vernachlässigbar erscheinen.
} 
Wir greifen an dieser Stelle die qualitative Abschätzung des Feldverlaufes in Gleichung 5.7 für die Feldstärke $E_{2}$ auf.

$$
E_{2} \propto \frac{U}{r}
$$

Dabei bezeichnet $a$ wieder den Kontaktradius zwischen Spitze und Probe. Für die ungefähre Abhängigkeit der Stärke des Feldgradienten an der Domänenwand von deren Ausdehnung und der anliegenden Spannung erhalten wir somit die Abschätzung

$$
\left.\nabla E\right|_{r} \propto \frac{U}{r} .
$$

Der Betrag des elektrischen Feldgradienten verhält sich also reziprok zum Domänenradius und proportional zur anliegenden Spannung. ${ }^{\mathrm{k}}$

\section{Zeitabhängigkeit gemäß „Kriechformel“}

Kriechphänomene sind eine Konsequenz des Widerstreites elastischer Kräfte, welche glatte Domänenwände begünstigen, und ihrer Wechselwirkung mit Haftpotentialen. Letztere können einerseits aus statistisch verteilten Defekten resultieren und damit einen ungeordneten Charakter besitzen, es kann aber auch eine kristalline Ordnung vorliegen, etwa wenn man in jeder Einheitszelle ein Haftzentrum sehen kann. Dies ist beispielsweise bei der Ausbreitung einer ferroelektrisch polarisierten Domäne der Fall.

Die Theorie des Domänenkriechens, ${ }^{[155]}$ welche insbesondere im Kontext der Dynamik von Flussschläuchen in Supraleitern 2. Art große Aufmerksamkeit erfährt, ist äußerst komplex und kann im Rahmen der vorliegenden Arbeit nicht behandelt werden. Wir bedienen uns hier lediglich der etablierten Kriechformel:

$$
\frac{d t}{d r} \propto \exp \left(G\left(\frac{\nabla E_{0}}{\left.\nabla E\right|_{r}}\right)^{\mu} / k_{\mathrm{B}} T\right) .
$$

Hierbei steht $G$ für die zugrundeliegende Energieskala, vorgegeben vor allem durch das Muldenpotential an den Haftzentren, und $\nabla E_{0}$ für einen kritischen Wert der ,treibenden Kraft", hier des elektrischen Feldgradienten. ${ }^{l}$ Der dynamische Exponent $\mu$ ist abhängig von der Natur des Haftpotentials, der Dimensionalität des Systems und der Art der involvierten elastischen Kräfte. Angesichts der Domänengröße, die die Schichtdicke keinesfalls deutlich übersteigt, können die Domänenwände als zweidimensionale Objekte angesehen werden. Im Falle eines periodischen Pinning-Potentials können wir dann einen dynamischen Exponenten $\mu \approx 1$ erwarten. ${ }^{144,155]}$ Anzeichen für zusätzliche, nicht gitterperiodische Haftphänomene an strukturellen Defekten werden in Abschnitt 5.4.2 besprochen.

\footnotetext{
${ }^{\mathrm{k}}$ Interessanterweise ist die vorliegende Proportionalität des Feldgradienten zu $U / r$ auch für das elektrische Feld gemäß Gleichung 5.7 selbst gültig. Die Untersuchung des Kriechverhaltens würde also in ähnlicher Form auf ein mit elektrischen Dipolmomenten wechselwirkendes $E$-Feld übertragen werden können; die Analyse des Kriechverhaltens ergibt so ein allgemeineres Kriterium für einen strukturellen Prozess als nur im Rahmen des oben vorgestellten Modells.

${ }^{1}$ Die Schwelle $\nabla E_{0}$ entsteht bereits aus der Natur des Kriechphänomens und ist damit von anderer Bedeutung als die zusätzlich vorliegende Schwellspannung $U_{\mathrm{C}}$. Ohne Schwellspannung wäre unterhalb von $\nabla E_{0}$ sonst durchaus noch ein langsames Domänenwachstum beobachtbar.
} 
Nach Einsetzen von 5.11 in die Kriechformel 5.12 und Integration über $r$ gelangt man durch Invertieren zu folgender Abhängigkeit:

$$
\frac{r}{U}=\alpha \log \left(\frac{\beta t}{U}+1\right) .
$$

Die Eins im Argument des Logarithmus entstammt dabei einer besonderen Wahl der Integrationskonstanten, welche die Randbedingung $r(t=0)=0$ erfüllt, also dem Vernachlässigen einer kritischen Nukleationsgröße der metallischen Inseln. Es wurde stattdessen von einem kontinuierlichen Wachstum ab dem Pulsbeginn und ohne einen bereits zuvor existierenden metallischen Bereich ausgegangen. Solche metallischen Nukleationskeime, also leitfähige Flecken im Ausgangszustand, lagen insbesondere in dem hier besprochenen Experiment ja nicht vor.

\subsubsection{Anwendung auf die experimentellen Ergebnisse}

Das strukturelle Modell ergibt, in Kombination mit der Kriechtheorie, eine logarithmische Zeitabhängigkeit des Domänenradius von der Dauer des Spannungspulses. Gemäß Gleichung 5.13 würde man ferner eine im Wesentlichen lineare Abhängigkeit von der Pulsspannung erwarten.

\section{Abhängigkeit der erzielten Domänengrößen im Pulsrasterexperiment}

Tatsächlich hatte die numerische Auswertung des in Abb. 4.12 gezeigten Pulsrasterversuches mittels eines MATLAB-Algorithmus auf eben diese Abhängigkeiten hingewiesen (vgl. Abb. 4.13). Eine Auftragung der relativen, das heißt mit der Spannung normierten, Domänenradien gegen die relativen Pulsdauern für alle metallischen Regionen ist in Abb. 5.7 gegeben. Sie bestätigt, trotz einer gewissen Streuung, auf eindrucksvolle Weise das universale Wachstumsverhalten gemäß der Kriechformel. Ein zum Vergleich eingetragener linearer und, der Vollständigkeit halber auch ein wurzelförmiger Fit zeigen eindeutig, dass ein simpler Elektromigrations- oder Diffusionsprozess mit dem beobachteten Wachstumverhalten unvereinbar ist. Die beiden Fitfunktionen wurden dabei bewusst ohne Ordinatenabschnitt gewählt, so dass, wie für den logarithmischen Fit, die Randbedingung $r(t=0)=0$ erfüllt wird. Andernfalls ließe sich zwar eine sehr viel bessere Übereinstimmung der Fitfunktion mit den ermittelten Radien erreichen, die aber wieder einer endlichen Domänengröße vor Pulsbeginn entspräche und somit dem experimentell nachgewiesenen, isolierenden Ausgangszustand widerspricht. Ferner wurde auch hier die Anwendbarkeit auf die relativen Radien und Pulsdauern, also eine näherungsweise lineare Spannungsabhängigkeit, vorausgesetzt.

Die numerische Analyse der Domänenradien ergab also ein Wachstumsverhalten, welches in seiner Spannungs- und Zeitabhängigkeit mit den Erwartungen gemäß der Kriechformel übereinstimmt. Dieses Resultat ist deshalb ein starkes Indiz für das Bild einer sich ausbreitenden strukturellen Änderung.

\section{Domänenrückbildung}

In Abschnitt 4.6.1 wurde gezeigt, dass durch Spannungspulse nicht nur langzeitstabile metallische Domänen entstehen, sondern sich auch auf sehr verschiedenen Zeitskalen Rück- 


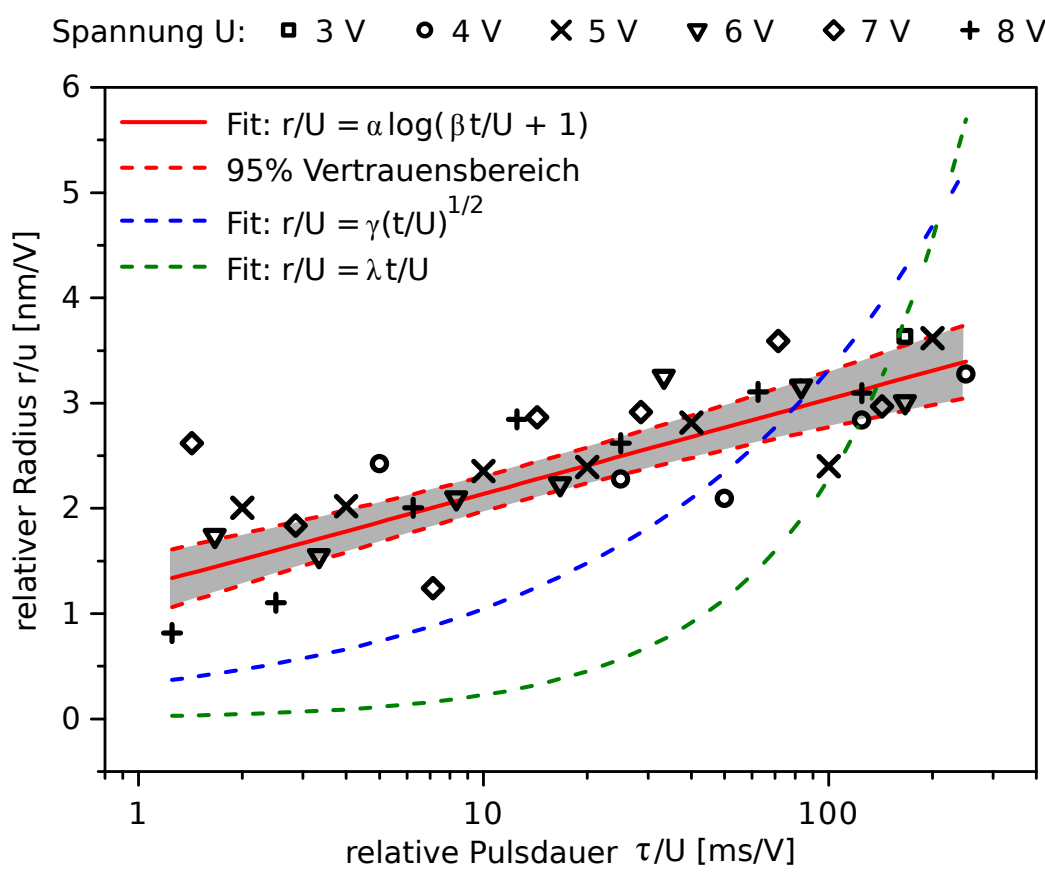

Abbildung 5.7: Domänenwachstum gemäß Kriechformel. Einzig die auf ein Domänenwandkriechen hinweisende, logarithmische Zeitabhängigkeit ist zur Beschreibung der Messdaten geeignet. Die Fitparameter betragen $\alpha=(0,39 \pm 0,05) \mathrm{nm} / \mathrm{V}, \beta=(24 \pm 18) \mathrm{V} / \mathrm{ms}$, $\gamma=(0,33 \pm 0,033) \mathrm{nm} /(\mathrm{V} \cdot \mathrm{ms})^{1 / 2}$ und $\lambda=(0,023 \pm 0,004) \mathrm{nm} / \mathrm{ms}$.

bildungen ereignen können. Die Aufnahme einer sehr langsamen Rückbildung ließ dabei ebenfalls eine Beschreibung durch ein logarithmisches Zeitgesetz zu. Auch dieses Ergebnis kann als Argument für das strukturelle Szenario angesehen werden: Die Existenz einer von ihrer Umgebung sich strukturell unterscheidenden Region ist mit energetisch ungünstigen Verspannungen an der Domänenwand verbunden. Demnach ist eine langsame Rückbildung also durchaus zu verstehen. Ob diese jedoch auch einer logarithmischen Zeitabhängigkeit folgen würde, ist schwer zu beurteilen. Anstelle des elektrischen Feldgradienten, beziehungsweise dem Sprung im elektrischen Feld, müsste als treibende Kraft jetzt die langreichweitige Verspannung als Funktion des Domänenradius untersucht werden. Man würde, ganz analog zu der Beschreibung magnetischer Domänen, vermuten, dass die Relation zwischen Oberflächen- und Volumenenergie sowie das Haften von Domänenwänden an Defekten einen entscheidenden Einfluss auf das Überleben der Domänen und damit die Remanenz des Schaltens haben. ${ }^{\mathrm{m}}$

Falls hingegen das ionische Bild zuträfe, so würde ohne anliegendes elektrisches Feld eine rein konzentrationsgetriebene Rückdiffusion für die Auflösung der metallischen Insel verantwortlich sein müssen. Diese würde nicht nur einen gewissen Transport in tiefere Schichten, welche möglicherweise zugunsten der entstandenen Insel eine Sauerstoffverarmung erfuhren,

\footnotetext{
${ }^{m}$ Man könnte in Abb. 4.16 auch zwei lineare Abnahmen unterschiedlicher Geschwindigkeit vermuten - beispielsweise dadurch verursacht, dass bei großer Ausdehnung der Domäne auch senkrecht zur Schichtebene anfangs noch Substrateffekte eine Rolle spielen.
} 
bedeuten. Vielmehr wäre, insbesondere für große metallisch geschaltete Regionen, auch ein lateraler Transport in die isolierende, sauerstoffdefizitäre Umgebung zu erwarten. Demzufolge würde man - für großflächiger umgeschaltete Regionen - eher eine Ausschmierung der Phasengrenze erwarten. Diese verläuft zwar tatsächlich nicht in allen Fällen scharf dies könnte im Rahmen unseres Modells auch durch ein ausgedehntes Verspannungsfeld mit langsamer struktureller Anpassung verstanden werden - ein Zerfließen, wie man es für ein Konzentrationsprofil erwarten würde, konnte jedoch nicht beobachtet werden. Die Existenz und Dynamik nanoskaliger Domänen mit relativ scharfer Phasengrenze ist jedoch an sich schon schwer mit der Vorstellung eines intuitiv eher kontinuierlich verlaufenden Konzentrationsprofils an Sauerstoffvakanzen zu vereinen.

\section{Leitfähigkeitsanstiegs- und -abklingverhalten in Pulsserienversuchen}

Die unter 4.6.2 vorgestellten Ergebnisse der Pulsserienversuche lassen sich in das obige Bild nahtlos einfügen. Abb. 5.8 illustriert, wie sich diese Messungen im strukturellen Bild als Kriecherholungsverhalten verstehen lassen: Während der positiven Spannungspulse erfolgt, wie im ersten Teil dieses Unterkapitels gezeigt, ein logarithmisches Domänenwachstum. In der Pause zwischen den Pulsen findet hingegen eine Abnahme der Leitfähigkeit, das heißt ein Schrumpfen der metallischen Insel statt. Durch Pulsserien mit kurzen Abständen kann jeder Puls den Überrest der noch nicht vollständig verschwundenen Domäne erneut vergrößern, so dass die Leitfähigkeit mit fortschreitender Pulszahl weiter ansteigt. Umgekehrt bedeutet ein zu langes Warten, dass die Rückkehr zum isolierenden Zustand vollendet wird und der nachfolgende Spannungspuls eine metallische Region aufs Neue erzeugen muss. Somit lässt sich keine Zunahme der Leitfähigkeit mehr erkennen.

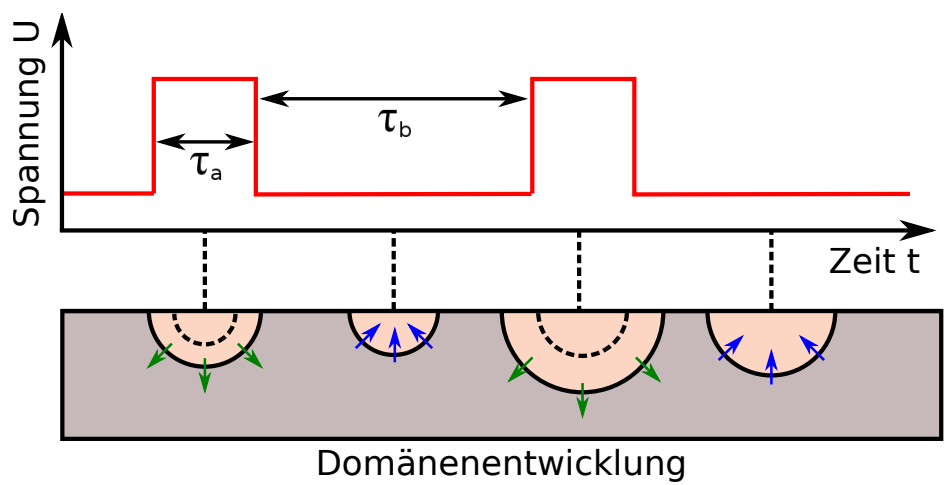

Abbildung 5.8: Hypothetische Domänenentwicklung im Verlauf von Pulsfolgen. Ein abwechselndes Wachsen während und Schrumpfen zwischen den Pulsen ergibt eine sich nur langsam ausdehnende metallische Region.

Interessanterweise wurden alle zum Zeitverhalten gezeigten Ergebnisse an derselben Probe gewonnen - sowohl die anscheinend langzeitstabilen Resultate des Pulsrasterexperimentes wie auch die Beobachtung des zeitlichen Abklingverhaltens. Über die Ursache kann hier nur spekuliert werden: Im Szenario der sich im elektrischen Feld ereignenden, propagierenden Strukturänderung erscheint ein starker Einfluss von Defekten wahrscheinlich. Strukturdefekte, wozu letztendlich auch die Oberfläche selbst und ihre Morphologie zählen, 
könnten als Haftzentren eine verspannungsbedingte Rückbildung verhindern und damit die oft beobachtete Remanenz des Schalteffektes bewirken. Umgekehrt würde gerade eine defektarme Schicht eine Rückbildung zum als energetisch günstiger anzunehmenden HRS erleichtern.

In Abb. 4.17 (b) wurden starke Variationen der einzelnen Stromverläufe während der Spannungsfolgen gezeigt. Die Ursache der teils langsamen, teils sprunghaften Änderungen der Leitfähigkeit kann hier nicht im Detail untersucht werden. Bemüht man allerdings noch einmal die Analogie zwischen unserer strukturellen Hypothese und dem ferromagnetischen Domänenwachstum, so lässt sich eine gewisse phänomenologische Ähnlichkeit mit dem magnetischen Barkhauseneffekt feststellen: Genau wie die sprunghafte Magnetisierungsänderung beim Überkommen des Haftpotentials an einem Gitterfehler durch eine vom externen Magnetfeld getriebene Domänenwand verstanden werden kann, könnte hier auch ein abrupter Leitfähigkeitsprung bei dem Durchlaufen eines Defektes durch die strukturelle Domänenwand verstanden werden. ${ }^{\mathrm{n}}$

Um eine tatsächliche Aufklärung dieses Sachverhaltes zu erreichen, müsste man allerdings gezielt den Einfluss von Defektdichten und Oberflächeneigenschaften auf die lokalen Schalteigenschaften untersuchen. Eine systematische Untersuchung ist jedoch im Rahmen dieser Arbeit keinesfalls zu leisten. Es seien allerdings die Ergebnisse von Muenstermann et al. erwähnt, welche für $\mathrm{SrTiO}_{3}$ bereits auf große Einflüsse der Probenmorphologie hinwiesen. ${ }^{[141]}$

Auch sollte man bei der Konzeption einer möglichen Anwendung des resistiven Schaltens als RRAM diesen Erfahrungen Rechnung tragen und die Untersuchungen mit lokalen Methoden intensivieren. Im Falle ausgedehnter Kontaktflächen ist es denkbar, dass nur auf einem kleinen Flächenanteil remanente Widerstandsänderungen erzielt werden können. Für eine Verwendung als nonvolatiles Speicherelement könnte daher die gezielte Kontrolle der Defektdichte erforderlich werden, um ausreichend hohe Speicherdichten zu erreichen.

\subsection{Einfluss von undotierten Decklagen}

Motivation für die unter 4.7.1 aufgeführten Messungen war der bekannte Einfluss weniger zusätzlicher Monolagen undotierten Lanthanmanganats an den Grenzflächen von ManganatTitanat-Heterostrukturen auf die strukturellen und elektrischen Eigenschaften. Wie bereits im Einführungskapitel erwähnt, stellen die Grenz- und Oberflächen für perowskitische Manganate eine empfindliche Störung des gesamten hochkorrelierten Systems dar. Der Symmetriebruch kann durch eine Schwächung des Doppelaustauschmechanismus eine massive Beeinträchtigung des metallischen Transportes und der ferromagnetischen Ordnung bewirken.

Experimentelle Befunde an Manganat-Titanat-Schichtsystemen durch Yamada et al. zeigten bereits eindeutig, dass wenige undotierte Monolagen an der Grenzfläche diese Effekte kompensieren und die ferromagnetische Ordnung wiederherstellen können. ${ }^{[56]}$ Darüberhinaus zeigten Arbeiten von Gehrke et al., dass sogar die Schichten fernab der

\footnotetext{
${ }^{\mathrm{n}}$ Die Beobachtung eines gewissen „Gedächtniseffektes“, dass nämlich zwei aufeinanderfolgende positive Pulsreihen nicht immer den gleichen Verlauf aufweisen, ist möglicherweise auf eine thermische Ausheilung oder Erzeugung von kleineren Defekten zurückzuführen. Ebenso wäre eine gewisse Drift zwischen Cantilever und Probe denkbar.
} 
Grenzfläche diese Modifikation spüren, und sich eine veränderte Struktur mit höherer Leitfähigkeit einstellt. ${ }^{[77,78]}$ Theoretische Betrachtungen von Brey untermauern dieses Konzept; es wird davon ausgegangen, dass man so die sich sonst an der Grenzfläche intrinsisch einstellende effektive Überdotierung korrigieren kann. ${ }^{[6]}$ Frühere Arbeiten von Calderón et al..$^{[74]}$ erklärten die Ausbildung toter Lagen an Manganatoberflächen durch ähnliche Prozesse. Daher erschien hier der Versuch einer Beeinflussung der Oberfläche durch zusätzliche LMO-Lagen sinnvoll.

Die Ergebnisse zeigten eine sehr homogen verteilte metallische Leitfähigkeit, wie sie an keiner anderen in dieser Arbeit untersuchten Probe vorlag. Im Rahmen des strukturellen Modells lassen sich diese Beobachtungen mühelos beschreiben: Die zusätzlichen, undotierten Lagen kompensieren die durch ein Sauerstoffdefizit verursachte Überdotierung der Oberfläche. Auf diese Weise wird eine Abschwächung des Doppelaustauschs verhindert und die Ausbildung weniger verzerrter struktureller Verhältnisse begünstigt. Ein metallischer Zustand resultiert dann aus der Reduktion der Mn-O-Mn-Bindungswinkel. ${ }^{\circ}$

Man versteht also intuitiv die Existenz eines Ausgangszustandes mit im Vergleich zu normalen Proben deutlich erhöhter Oberflächenleitfähigkeit. Die Möglichkeit, diesen Zustand durch Anlegen großer elektrischer Felder im Nachhinein zu modifizieren, worauf erste (nicht gezeigte) Messungen hindeuten, lässt sich im strukturellen Bild so begreifen, dass die Verzerrung durch einen Elektronentransport von der Oberfläche weg wieder verstärkt werden kann. Während die Terminierung durch undotiertes LMO nämlich die statische räumliche Anordnung der A-Platz-Ionen betrifft, ist die im Modell enthaltene elektronische Ladungsverschiebung, welche durch eine Änderung der jeweiligen lokalen Dotierung verschiedene strukturelle Phasen fördern kann, hiervon teilweise entkoppelt - die elektronische Ladungsverteilung lässt sich durch starke elektrische Felder beeinflussen. Man erwartet also nicht unbedingt, dass ein metallischer Ausgangszustand nicht ebenso veränderlich ist wie ein isolierender, sondern beginnt nur mit einer a priori verschobenen Balance der beiden Kontrahenten zugunsten der metallischen Phase.

Bedient man sich nun stattdessen des ionischen Szenarios, so erscheint es völlig unerklärlich, warum die Terminierung mit Lanthanmanganat einen Ausgleich der Oberflächenstöchiometrie bewirken sollte. Unter vergleichbaren Wachstumsbedingungen würde man eher eine ähnlich sauerstoffdefizitäre und somit isolierende Oberfläche erwarten. Die Kompensation der Fehldotierung würde zwar auch hier eine erhöhte Grundleitfähigkeit bewirken können, man würde aber im Ausgangszustand noch immer das isolierendere Verhalten erwarten. Die nicht gezeigten vorläufigen Ergebnisse deuten aber darauf hin, dass ein hochohmigerer Zustand durch das Anlegen negativer Spannung erzeugt werden kann.

Weitere experimentelle Untersuchungen der LMO-terminierten Oberflächen könnten sich als sehr aufschlussreich erweisen. In Verbindung mit TEM-Querschnitten zur hochauflösenden Strukturuntersuchung der modifizierten oberflächennahen Bereiche könnte

\footnotetext{
${ }^{\circ}$ Es ist wieder zu beachten, dass der Begriff „,metallisch“ hier nicht zwangsläufig mit dem ferromagnetischmetallischen Tieftemperaturzustand des Volumenmaterials gleichzusetzen ist. Es wird nur von einer Verringerung der zusätzlichen Verzerrung ausgegangen, welche sich sonst möglicherweise an der Oberfläche ausbildet. Die „metallische“ Leitfähigkeit muss dabei die des bei Raumtemperatur ja isolierenden Volumenzustandes nicht unbedingt übertreffen, sondern nur die der besonders hochohmigen Oberflächenregion.
} 
eine detaillierte Analyse des Schaltverhaltens vielleicht wertvolle Informationen über die Richtigkeit der Hypothese eines strukturellen Mechanismus liefern.

Auch für mögliche Speicheranwendungen sind diese Ergebnisse eventuell von großer Relevanz: Die Möglichkeit einer selbstständigen Rückbildung metallischer Domänen würde ein Problem für remanent schaltbare Bauelemente darstellen. Nonvolatile Speichertechnologien könnten deshalb sehr von einem derartigen ,interface engineering“ profitieren, um die Balance der Energiedichten beider Widerstandszustände gezielt zu verbessern.

\subsection{Diskrete Leitfähigkeitsplateaus}

In Abschnitt 4.7.2 wurden Indizien dafür gezeigt, dass eine LSMO-Oberfläche beim elektrisch induzierten Schalten nur diskrete Widerstandszustände annehmen kann.

Im elektrochemischen Modell ist ein derartiges Verhalten nur sehr schwer zu verstehen; in diesem Beschreibungsversuch ist die Leitfähigkeit mit der Sauerstoffleerstellendichte verknüpft. Der vorliegende Befund diskreter Leitfähigkeitswerte würde dementsprechend die bevorzugte Einstellung spezifischer Leerstellendichten bedeuten. Diese müsste ferner über große Flächen nahezu konstant vorliegen und sich dann stellenweise abrupt ändern. Typischerweise würde man stattdessen eher eine kontinuierliche Variation der Leerstellenkonzentration erwarten, die auch räumlich einen glatteren Verlauf aufweisen sollte. Einzig die Feststellung, dass höhergelegene Regionen isolierender sind, ließe sich insofern mit dem Bild der Sauerstoffdrift in Einklang bringen, als ein vor allem oberflächennahes Sauerstoffdefizit existiert, welches sich in einer Reduktion der Leitfähigkeit von Erhebungen besonders bemerkbar machen könnte. Die diskreten Leitfähigkeitswerte lassen jedoch auch hier, ebenso wie die laterale Ausbreitungsform, erhebliche Zweifel an einer Anwendbarkeit des ionischen Szenarios aufkommen.

Wie aber kann man sich dieses Verhalten im strukturellen Bild erklären? Wir beginnen mit einem Vergleich verschiedener Manganatsysteme. Für PCMO, welches in orbital ungeordneten Bereichen eine ausgeprägte orthorhombische Verzerrung (Pnma) aufweist, konnte von Jooss et al. durch TEM-Messungen gezeigt werden, dass sich einhergehend mit einer elektrisch induzierten Widerstandsänderung auch eine Ausbreitung beziehungsweise Zerstörung von verschiedenen niedersymmetrischen Überstrukturen in ladungs- und orbitalgeordneten Domänen ereignet. ${ }^{[56,106]}$ Inkommensurable elektrisch induzierte Überstrukturen können sich auch bis in Bereiche, die über $90 \mathrm{~nm}$ weit von der Grenzfläche entfernt sind, erstrecken.

Die Struktur von LCMO ist nicht so stark verzerrt, wodurch sich bereits ein reduzierter spezifischer Volumenwiderstand ergibt. Die Annahme einer isolierenden Decklage ist für dieses System verbreitet. ${ }^{[157]}$ Man kann sich daher vorstellen, dass diese Oberflächenlage zu einem metallischen Zustand schaltet, dessen Leitfähigkeit und strukturelle Symmetrie die des nativen Volumenzustands eventuell sogar noch übersteigen. Daher ist auch hier, wie bei der Modellierung des Domänenwachstums in Abschnitt 5.4 angenommen, eine gewisse Ausbreitung der metallischen Domäne in die Tiefe der Schicht denkbar.

LSMO liegt demhingegen auch bei Raumtemperatur im Volumen in einer rhomboedrischen Konfiguration mit metallischen Eigenschaften vor. Die Oberfläche ist, wie es die C-AFMErgebnisse eindeutig zeigen, im Ausgangszustand trotzdem stark isolierend, also vermutlich 


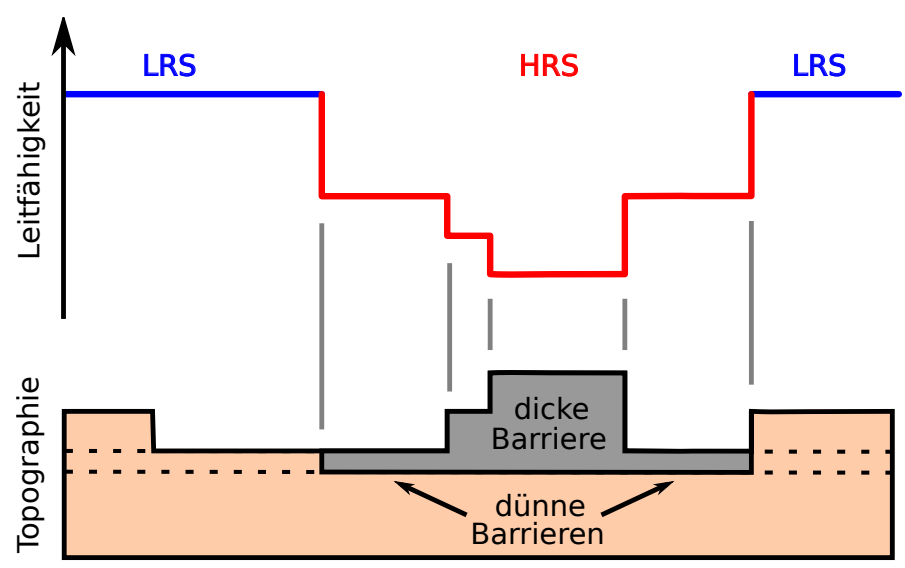

Abbildung 5.9: Diskrete Leitfähigkeitsplateaus als Folge monolagenweisen Umschaltens (schematisch). Die mit der Topographie korrelierten, anscheinend diskreten Leitfähigkeitswerte (siehe Abschnitt 4.7.2) lassen sich möglicherweise durch die Annahme einer charakteristischen Tiefe, bis zu welcher sich eine isolierende Deckschicht ausbilden kann, verstehen. Die darüberliegende glatte, sich nur über wenige Lagen erstreckende Topographie ergäbe eine in diskreten Schritten verlaufende Barrierendicke.

ladungsgeordnet und Jahn-Teller-verzerrt. Ein strukturelles Schalten sollte demnach eher auf die Oberflächenlagen selbst beschränkt sein und nur durch eine Anpassung an die innere, pseudokubische Struktur erfolgen.

Als Erklärungsansatz ist es daher naheliegend, den Widerstand der jeweiligen Oberflächenregion einer unterschiedlichen Dicke der dortigen isolierenden Deckschicht zuzuschreiben. Die Existenz diskreter Widerstandszustände entspräche dann dem Vorliegen isolierender Barrieren definierter Dicken. Rein hypothetisch kann man sich sogar ein schrittweises Umschalten einzelner Monolagen vorstellen - natürlich gibt es dafür ob der begrenzten C-AFM-Auflösung jedoch keine experimentellen Beweise. Es ist allerdings bemerkenswert, dass die untersuchte Probenregion tatsächlich sehr glatt ist und sich die Höhenskala nur über wenige Gitterkonstanten erstreckt.

Die beobachteten Korrelationen zwischen der Topographie und der lokalen Leitfähigkeit ließen sich, wie in Abb. 5.9 illustriert, in diesem spekulativen Bild folgendermaßen verstehen: Vor Anlegen der negativen Pulse ${ }^{\mathrm{P}}$ lag die gesamte Oberflächenregion im metallischen Zustand vor, das heißt sie ist strukturell hochsymmetrisch und eine eventuelle effektive Fehldotierung wurde behoben.

Beim Zurückschalten wird die Oberfläche bis zu einer charakteristischen Tiefe wieder isolierend geschaltet. Die darüberliegenden Erhebungen - welche hier von der Stärke nur weniger Atomlagen und daher wahrscheinlich nicht deutlich dicker als die isolierende Schicht darunter sind - werden ebenfalls isolierend. Selbst wenn sie zur Zeit des Spannungspulses nicht an der Position der Cantileverspitze liegen, ist ihr Rückgang in den isolierenden Zustand energetisch günstig, um die aufwendige Ausbildung weiterer Grenzflächen zu

${ }^{\mathrm{p}}$ Es sei auf die Vorgeschichte der diskutierten Messung hingewiesen: Die Probenregion wurde zuvor durch permanente positive Spannung metallisch geschaltet, darauf folgten wenige lokale negative Pulse. 
vermeiden. ${ }^{\mathrm{q}}$ Auf diese Weise ergäben sich somit isolierende Barrieren unterschiedlicher diskreter Dicken. Es sei an dieser Stelle ergänzend auf die Diplomarbeit von Christin Kalkert hingewiesen, worin mittels Rastertunnelspektroskopie eine Phasenseparation in der Oberflächenleitfähigkeit einer LPCMO-Schicht aufgezeigt wird. ${ }^{[158]}$ Auch diese weist Korrelationen mit der Topographie auf, welche vermutlich in einem Verspannungsabbau durch die Lage der Domänenwände an den Terrassengrenzen begründet sind.

${ }^{\mathrm{q}}$ Diese Vorstellung erscheint auch deshalb plausibel, weil es sich hier um eine sehr glatte Oberflächenregion handelt. An rauheren Stellen könnte möglicherweise das andere Oberfläche-zu-Volumen-Verhältnis ein Umschalten des gesamten Hügels verhindern. 



\section{Kapitel 6 Zusammenfassung und Ausblick}

Abschließend werden die wesentlichen Inhalte dieser Arbeit noch einmal zusammenfassend dargestellt. Es folgen eine kurze Einordnung der Ergebnisse in Bezug auf die technische Nutzbarkeit der untersuchten resistiv schaltenden Materialien in der Speichertechnologie und ein Ausblick, welche Ansatzpunkte sich für zukünftige Untersuchungen anbieten.

\subsection{Zusammenfassung}

In dieser Arbeit wurde das spannungsinduzierte, remanente und reversible Widerstandsschalten von Manganatoberflächen untersucht. Dabei kam die C-AFM-Methode zum Einsatz, um den Schalteffekt auf der Nanoskala untersuchen zu können. An einer Reihe mittels der metallorganischen Aerosoldeposition hergestellter epitaktischer Proben fand sich - im wesentlichen dotierungsunabhängig - ein qualitativ sehr ähnliches Verhalten: Die Leitfähigkeit der im Ausgangszustand hochisolierenden Oberfläche wird ab einer positiven kritischen Spannung, welche in keinem Fall unter etwa $U_{\mathrm{C}}=3 \mathrm{~V}$ lag, drastisch erhöht.

Der so erzeugte „metallische“ Zustand der Probenoberfläche kann langfristig überdauern, der isolierende Zustand lässt sich dann durch das Unterschreiten einer negativen Schwellspannung wiederherstellen; davon abweichend wurde jedoch auch eine selbstständige Regeneration des hochohmigen Verhaltens beobachtet. Die Ergebnisse lassen sich durch das in der Literatur intensiv diskutierte Bild der Elektromigration von Sauerstoffleerstellen nicht befriedigend erklären. Ein struktureller Übergang im elektrischen Feld erscheint im vorliegenden Falle plausibler. Es wurde ein einfaches phänomenologisches Modell vorgestellt, um diese Annahme zu illustrieren. Demnach können elektrische Felder sich an der Oberfläche ausbildende Ladungsmomente angreifen, so eine Ladungsverschiebung hervorrufen und, sofern die Jahn-Teller-Energie überwunden werden kann, einen strukturellen Übergang induzieren. Obgleich die Details des hypothetisch angenommenen strukturellen Mechanismus unklar bleiben, konnte eine Reihe von Indizien aufgefunden werden, welche die Richtigkeit der Vorstellung vom strukturellen Übergang nahelegen und mit einem auf Elektromigration basierenden Prozess nur schwer vereinbar sind.

Während die sich gelegentlich ereignenden drastischen Modifikationen der Oberflächenmorphologie wahrscheinlich auf thermische Schädigungen der Cantileverspitze und der Probe zurückgeführt werden können, erscheinen die vorherrschenden Stromdichten und 
Jouleschen Erwärmungen oftmals als zu klein, um die in Manganaten geringe Sauerstoffmobilität entscheidend zu fördern. Auch ist die Existenz einer scharfen Spannungsschwelle im strukturellen Bild leicht begreifbar, erscheint aber im ionischen Szenario nicht plausibel. Insbesondere das als Kriechphänomen erklärbare logarithmische Wachstumsverhalten von metallischen Domänen unter anliegender Spannung stützt das strukturelle Szenario.

Die Untersuchung von LMO-terminiertem Material zeigte darüberhinaus einen im Elektromigrationsmodell nicht verständlichen metallischen Ausgangszustand. Der hochinteressante Befund anscheinend diskreter, mit der Topographie korrelierter Leitfähigkeitszustände einer glatten LSMO-Oberfläche muss als Indiz für eine sich lagenweise ausbreitende Strukturänderung verstanden werden und kann im Rahmen des ionischen Migrationsmodells nicht erklärt werden.

Obwohl die mikroskopischen Details des Schaltprozesses weiterhin unklar sind, bestehen demnach ernste Zweifel an der Richtigkeit der diffusiven Beschreibung von Schaltphänomenen - zumindest im Bezug auf die hier behandelten Manganate. Ein struktureller Übergang muss als wesentlicher Bestandteil des Schaltmechanismus in der Beschreibung berücksichtigt werden.

\subsection{Anwendungsrelevanz}

In Bezug auf technische Anwendungen zeigten die Ergebnisse einige mögliche Probleme in der angestrebten Konstruktion nonvolatiler Speicherelemente auf. Der beim RRAM unerwünschten selbstständigen Rückkehr metallischer Regionen in den isolierenden Zustand kann eventuell durch eine Kontrolle der Defektdichte und Oberflächenmorphologie zur Schaffung von Haftzentren oder sogar, eleganter, durch eine Terminierung geringerer Dotierung erzielt werden. Sehr vielversprechend ist die prinzipielle Robustheit des Schalteffektes bezüglich verschiedener Dotierungen und die schon jetzt erzielbare Speicherdichte: Die beobachteten typischen Domänengrößen von nur wenigen zehn Nanometern ergeben bereits eindrucksvolle Werte bis hin zu einigen Tbit/inch ${ }^{2}$. Tatsächlich sind solche nanoskaligen Messungen für Aufschlüsse über die Realisierbarkeit des RRAM-Konzeptes unverzichtbar, da die experimentellen geometrischen Bedingungen denen der angestrebten Konstruktion ähneln.

Für das sogenannte multilevel switching, welches mehr als nur binäre und eventuell sogar quasi-analoge Speicherelemente ermöglichen könnte, sind die diskreten Leitfähigkeitswerte von großer Bedeutung. Sie lassen vermuten, dass eine kontinuierliche Widerstandsänderung durch die dreidimensionale Domänenausbreitung zustande kommt. Dem lagenweise erscheinenden Umschalten über diskrete Widerstandszustände liegen aber wahrscheinlich nur zwei tatsächlich physikalisch beziehungsweise strukturell verschiedene Konfigurationen zugrunde.

Als problematisch sind die oftmals sehr langen Schaltzeiten im Millisekundenregime zu bewerten. Es gibt jedoch eine Vielzahl anderer Arbeiten, bei welchem mit Pulsen von einigen hundert Nanosekunden Länge bereits geschaltet werden konnte. ${ }^{[96,159]}$ Eine weitere Herausforderung könnte in der Vermeidung von Alterungsprozessen der Speicherelemente bestehen - die sich oftmals ereignenden gravierenden morphologischen Änderungen machten deutlich, wie nahe die bei den Schaltpulsen auftretenden Stromdichten an der Belastbarkeitsgrenze des Materials liegen. 


\subsection{Ausblick}

Diese Abhandlung hat zwar starke Argumente für die in der Literatur bisher nur unzureichend diskutierte Beteiligung struktureller Änderungen am Schaltprozess ergeben, ein direkter Beweis eines strukturellen Überganges konnte jedoch nicht erbracht werden. Zur weiteren Untersuchung dieser spannenden Frage nach den mikroskopischen Details beim resistiven Schalten müssen - wie es bereits durch TEM-Studien an PCMO geschieht auch für die hier behandelten Systeme elektronenmikroskopische Techniken zum Einsatz kommen, um die strukturelle Hypothese zu überprüfen. Dabei könnten sich insbesondere Beugungsmethoden - wie auch im optischen Bereich beispielsweise durch mikroskopische Raman-Techniken - als aufschlussreich erweisen. Eine große experimentelle Herausforderung besteht dabei in der meist nur sehr geringen lateralen und vertikalen Ausdehnung des schaltenden Bereichs.

Bei zukünftigen Studien muss außerdem dem Zeitverhalten der Schaltprozesse große Aufmerksamkeit gewidmet werden, da es, wie hier in ersten Analysen bereits ausgenutzt, wichtige Rückschlüsse auf den Mechanismus liefern kann. Zeitaufgelöste Messungen des Stroms, welcher die Domänengröße abbildet, können wesentlich zu einer Entscheidung beitragen, ob dem resistiven Schaltphänomen ein in der Regel nur langsamer diffusiver Prozess zugrundeliegt, oder ein struktureller Übergang, welcher prinzipiell eine größere Ausbreitungsgeschwindigkeit erlaubt: Der sehr schnellen elektronischen Anregung könnte eine strukturelle Änderung im Idealfall etwa mit Schallgeschwindigkeit folgen.

Darüberhinaus könnten sich aus Untersuchungen des Zeitverhaltens wertvolle Erkenntnisse darüber geben, ob sich eher ein lokales Schalten am Ort des größten Spannungsabfalls - beziehungsweise auch der Feldstärke oder des Feldgradienten - ereignet, oder größere Bereiche kooperativ umschalten. In letzterem Fall wäre die Dynamik vor allem durch pinning und depinning an Haftzentren geprägt und sollte sprunghafte Signaturen zeigen. Interessanterweise sollte eine große Dichte an Defekten so zwar die Stabilität gleichzeitig vorliegender, verschiedener struktureller Phasen begünstigen, aber für die Ausbreitung der strukturellen Änderung eher ein Hemmnis darstellen. Im Gegensatz dazu stehen die Erwartungen im ionischen Szenario: Hier müsste man durch die höhere lokale Ionenmobilität an Defekten eher eine vereinfachte Elektromigration, aber durch die ebenfalls erleichterte Eigendiffusion eine Verschlechterung der Remanenz vermuten.

Für weitere Untersuchungen empfiehlt sich ferner eine Betrachtung der Frequenzabhängigkeit - ein nichtlineares Verhalten könnte als Indiz für derartige ausgedehnte, kooperativ umschaltende Objekte angesehen werden. Wie bei anderen ungeordneten Systemen, beispielsweise den metallischen Gläsern, wo sich unter Zugspannung elastisch-plastische Übergänge ereignen, sollte eine detaillierte Analyse des Kriechverhaltens unter elektrischer Spannung den Übergang zwischen einem hochohmigen, polaronischen Transport einerseits und quasi metallischer Leitfähigkeit andererseits beleuchten können. 



\section{Anhang A Widerstands-Kapazitäts-Netzwerke}

Studien an Widerstandsnetzwerken, welchen neben zufälligen Verteilungen auch verschiedene mikroskopische Modelle zur Simulation eines Phasenseparationsbildes zugrundegelegt wurden, konnten schon große Erfolge bei der Beschreibung des Metall-Isolator-Überganges von Manganaten und dem kolossalen Magnetowiderstands-Effekt verzeichnen. Sie zeigen, dass die Transporteigenschaften dieser Systeme perkolative Züge tragen. ${ }^{[10,11,25,160,161]}$

Rairigh et al. belegten ferner durch ihre Beobachtungen eines kolossalen Magnetokapazitätseffektes an $\left(\mathrm{La}_{0,5} \mathrm{Pr}_{0,5}\right)_{0,67} \mathrm{Ca}_{0,33} \mathrm{MnO}_{3}$, dass nicht nur das Gleichstromverhalten, sondern auch die intrinsichen Kapazitätseigenschaften durch solche Perkolationsphänomene geprägt sind. ${ }^{[162]}$

Neben den im Hauptteil dargestellten Arbeiten zum resistiven Schalten von Manganaten wurden im Rahmen dieses Dissertationsprojektes auch in geringerem Umfang numerische Simulationen des frequenzabhängigen Transportverhaltens von Manganaten durch Widerstands-Kondensator-Netzwerke ( $R C$-Netzwerke) durchgeführt, welche in diesem Anhang erläutert werden. Die darin vorgestellte Methodik ist nur ein erster Versuch, um kapazitive Anteile in die für Manganatsysteme sonst sehr erfolgreichen Perkolationsrechnungen zu integrieren. Die vielversprechende qualitative Übereinstimmung erster Resultate mit Messergebnissen soll vor allem einen Anstoß für weiterführende Arbeiten geben.

\section{A.1. Motivation}

Ausgangspunkt für die folgenden Simulationen waren von Florian Fischgrabe durchgeführte Messungen des frequenzabhängigen Transports verschiedener epitaktischer Manganatproben. ${ }^{[163]}$ Während LSMO praktisch keine Frequenzabhängigkeit zeigte, also gewissermaßen als metallischer Leiter verstanden werden kann, ${ }^{a}$ war für LCMO in der Nähe des Metall-Isolator-Übergangs erkennbar, dass sich das Maximum im $R$ - $T$-Diagramm mit steigenden Frequenzen in ein flaches Minimum verkehrt. Es ist dabei zu beachten, dass eine

\footnotetext{
${ }^{a}$ Die sich beispielsweise im Drude-Modell ${ }^{[13]}$ ergebende Wechselstromleitfähigkeit nimmt zwar zu sehr großen Frequenzen $\omega$ hin ab, ist aber für die hier untersuchten Frequenzen $\omega \tau \ll 1$ quasi konstant. Die mittlere Relaxationszeit beträgt beispielsweise für $\mathrm{La}_{0,825} \mathrm{Sr}_{0,175} \mathrm{MnO}_{3}$ nach Lunkenheimer et al. $\tau=1,8 \times 10^{-14}$ s. $^{[164]}$
} 
apparative Kapazität, welcher der Probe beispielsweise als Kabelkapazität parallel zugeschaltet ist, einen solchen Verlauf nicht erklären kann. Eine solche parasitäre Zusatzkapazität wäre im wesentlichen temperaturunabhängig und könnte somit bei hohen Frequenzen nur eine Abflachung des Temperaturverlaufes bewirken. LPCMO zeigte die Ausbildung eines noch deutlicheren Minimums in der Nähe des Metall-Isolator-Übergangs - diese Messung ist in Abb. A.1 gezeigt. ${ }^{b}$

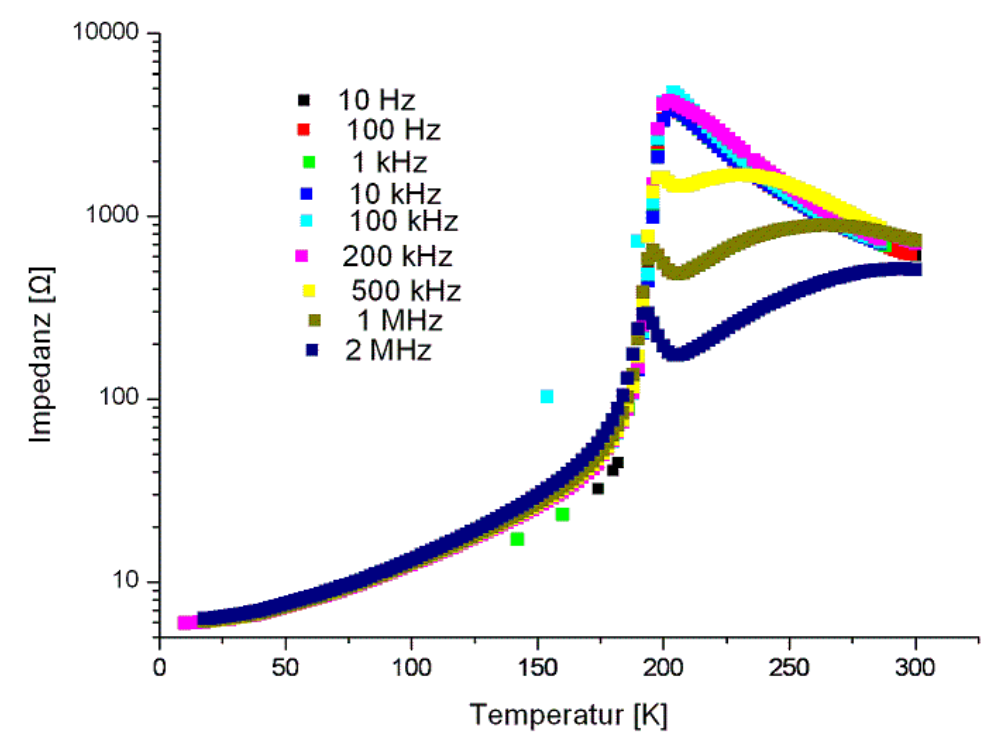

Abbildung A.1: AC-Transportverhalten einer $\left(\mathrm{La}_{0,6} \mathrm{Pr}_{0,4}\right)_{0,7} \mathrm{Ca}_{0,3} \mathrm{MnO}-\mathrm{Schicht}$. Aus [163].

Weil die Ausbildung eines solchen Impedanzminimums also eindeutig als intrinsischer Probeneffekt aufzufassen ist, liegt die Vermutung nahe, dass kapazitive Beiträge innerhalb der Probe das Hochfrequenzverhalten bestimmen. Dass diese Einflüsse sich in der Nähe des Metall-Überganges am bemerkbarsten machen, während sich das Transportverhalten für große und kleine Temperaturen dem Gleichstromfalle asymptotisch annähert, lässt eine Ursache in der Phasenseparation am Übergang vermuten. Unter dieser Annahme erscheint es auch plausibel, dass für LCMO nur schwache und für LSMO praktisch keine Effekte auftreten.

Die Grundannahme der hier vorgestellten Simulationen ist, dass sich eine geeignete Beschreibung darauf reduzieren lässt, den Grenzflächen zwischen metallischen isolierenden Bereichen im phasenseparierten Regime nahe des Übergangs eine Kapazität zuzuordnen. Wäre nämlich in einem Zweiphasenbild einer der beiden Kontrahenten permanent mit einer Kapazität behaftet, so müsste man auch für große beziehungsweise für kleine Temperaturen eine Frequenzabhängigkeit des Transportverhaltens auffinden. Eine dritte, nur in Übergangsnähe existierende Phase ist sicherlich auch denkbar. Tunnelspektroskopische Messungen

\footnotetext{
${ }^{\mathrm{b}}$ Neben den hier beschriebenen Ergebnissen für epitaktische Proben ist die im Folgenden dargestellte Methodik eventuell auch für solche Proben von Interesse, deren Eigenschaften durch interne Grenzflächen bestimmt werden. So zeigten beispielsweise von Christin Kalkert durchgeführte Untersuchungen des AC-Transportes nanokolumnarer LSMO-Schichten ein ähnliches Verhalten wie die epitaktische LPCMOProbe.
} 
von Christin Kalkert ergaben Indizien für die Existenz einer dritten Phase, welche aber ohnehin bevorzugt in den Übergangsbereichen zwischen den metallischen und isolierenden Regionen auftritt. ${ }^{[158]}$ Daher erscheint die Reduktion auf eine zusätzliche Kapazität allein an den Grenzflächen als sinnvolle Annahme.

\section{A.2. Knotenpotentialanalyse}

Die Knotenpotentialanalyse ist ein Standardverfahren der Elektrotechnik zur Analyse linearer Netzwerke. ${ }^{[165,166]}$ Das Prinzip ist relativ einfach: Es wird zunächst ein Gleichungssystem aus den Kirchhoffschen Knotengleichungen $\sum_{k} I_{k}=\sum_{k} G_{k} U_{k}=0$ aufgestellt. Der Strom $I_{k}$ durch jeden Zweig $k$ wird als Produkt aus dessen Leitwert $G_{k}$ und der Potentialdifferenz $U_{k}=\phi_{i}-\phi_{j}$ der beiden angrenzenden Knoten $i$ und $j$ dargestellt. Gesucht sind die Knotenpotentiale $\phi_{i}$, aus deren Kenntnis sich alle weiteren Größen ergeben. Legt man einen Knoten des Netzwerks auf einen festen Referenzwert (entsprechend einer Erdung), so ist dass Gleichungssystem lösbar. Während ideale Stromquellen direkt eingebunden werden können, tragen ideale Spannungsquellen eine weitere Unbekannte, den Quellenstrom, bei. Ein Lösungsweg besteht darin, eine der angrenzenden Knotengleichungen durch die Summe aus beiden zu ersetzen, wodurch der Quellenstrom aus dieser Gleichung eliminiert wird. Anstelle der anderen Knotengleichung tritt die bekannte Potentialbeziehung gemäß der Spannungsquelle. Die Lösung des Gleichungssystems geschieht dann durch Standardalgorithmen, hier mittels der mldivide-Routine von MATLAB.

Im Rahmen des Knotenpotentialverfahrens kann durch eine Komplexifizierung von Leitwerten und Strömen die Beschreibung leicht auf kapazitive beziehungsweise induktive Bauelemente erweitert werden. Die beiden folgenden Abschnitte beschreiben Ansätze, $R C$ Netzwerke mit verschiedenem Perkolationsverhalten zu generieren. Das Transportverhalten der erzeugten Netzwerke wird schlussendlich immer mit dem Knotenpotentialverfahren berechnet. $^{\mathrm{c}}$

\section{A.3. Zufallsnetzwerke}

Allen Simulationen lagen Netzwerke der in Abb. A.2 (a) dargestellten Topologie zugrunde. Die quadratische Anordnung besteht aus $N \times N$ Knoten, die jeweils mit ihren oberen, unteren und seitlichen Nachbarn über ein Impedanzelement verbunden sind. Die Knoten der rechten und linken Kante fungieren als Kontakte; sie sind über je ein weiteres Element mit der als ideal angenommenen Spannungsquelle verbunden. Die Strommessung zur Widerstandsbestimmung erfolgt für den Gesamtstrom durch das so kontaktierte Netzwerk.

Für Zufallsnetzwerke aus metallischen und isolierenden Widerständen $R_{\mathrm{M}}$ und $R_{\mathrm{I}}$ erhält man so auch hier das übliche zweidimensionale Perkolationsverhalten mit einer kritischen Konzentration $p=0,5$ des metallischen Anteils. Im Folgenden soll das Perkolationsverhalten eine Modifikation erfahren, welche frequenzabhängig zu Buche schlägt. Man könnte hierzu der

\footnotetext{
${ }^{c}$ Die hier gezeigten Simulationen wurden alle mit MatLAB durchgeführt. Weil sich sowohl die Umsetzung der Knotenpotentialanalyse als auch die verwendeten Monte-Carlo-Algorithmen an Standardprozeduren orientieren, wird auf eine Wiedergabe der Quelltexte der Programme, deren Umfang auch den Rahmen dieser Abhandlung sprengen würde, verzichtet.
} 
(a) Netzwerktopologie

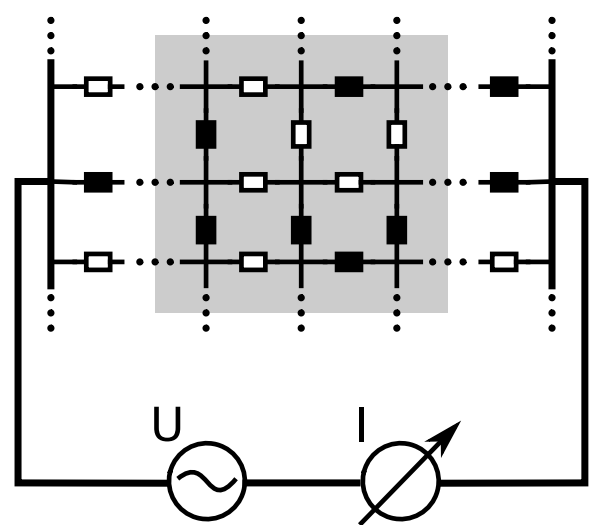

(b) Einbau von Kapazitäten

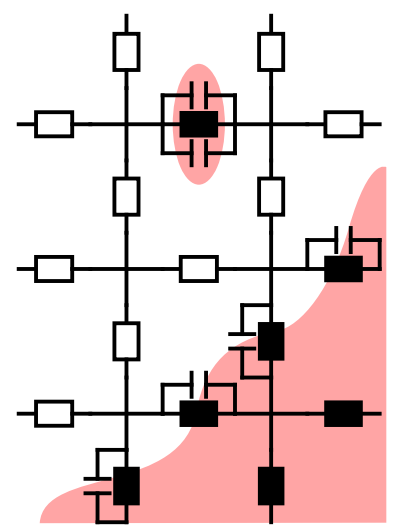

Abbildung A.2: Konstruktionsprinzip der $R C$-Netzwerke. (a) Ein quadratisches Netzwerk aus metallischen und isolierenden Widerständen wird durch je eine weitere Reihe an zwei gegenüberliegenden Kanten kontaktiert. Berechnet wird der Gesamtstrom durch dieses Netzwerk. (b) Isolatoren (ausgefüllt dargestellt), die an einem Knoten mindestens einen metallischen Nachbarn haben, werden durch eine Kapazität überbrückt. Diese Ergänzung wird für beide angrenzenden Knoten unabhängig durchgeführt. Die Kapazitäten verlaufen so im wesentlichen senkrecht zu den Grenzflächen zwischen isolierenden und metallischen Bereichen.

metallischen oder isolierenden Phase eine zusätzliche Kapazität zuordnen. Da diese jedoch im Tief- beziehungsweise Hochtemperaturbereich den Transport gegenüber der jeweils anderen Phase dominieren, würde man gerade dort eine ausgeprägte Frequenzabhängigkeit erhalten. Sind zum Beispiel die isolierenden Elemente mit einem Kondensator $C$ überbrückt, so wird der Ohmsche Widerstand bei hohen Temperaturen mit steigender Frequenz zunehmend kurzgeschlossen. Abb. A.1 zeigt aber vor allem im Bereich des Übergangs ein ausgeprägtes Frequenzverhalten, während sich die Widerstandskurven für große und kleine Temperaturen dem Gleichstromverlauf asymptotisch nähern.

Daher soll nun die angenommene Grenzflächenkapazität in das Zufallsnetzwerk eingefügt werden; das Vorgehen ist in Abb. A.2 (b) illustriert. Ein Suchalgorithmus prüft an jedem Knoten, ob dort metallische und isolierende Elemente aneinandergrenzen. ${ }^{\mathrm{d}}$ Falls dies der Fall ist, so wird den Isolatoren jeweils eine Kapazität parallel hinzugeschaltet. ${ }^{\mathrm{e}}$ Weil so alle Knoten abgearbeitet werden, erhalten solche Isolatoren, die an beiden Knoten jeweils mindestens einen metallischen Nachbarn besitzen, sogar zwei Kondensatoren. Es wäre zwar mit nur geringem Aufwand möglich, eine solche doppelte Überbrückung zu verhindern;

\footnotetext{
${ }^{\mathrm{d}}$ An Kanten des Netzwerkes wurden in der hier implementierten Fassung keine Kapazitäten ergänzt. Eine Erweiterung des Algorithmus ist hier ohne großen Aufwand möglich; für die hier durchgeführten ersten qualitativen Studien und angesichts der verwendeten Netzwerkgrößen sind die dadurch resultierenden Ungenauigkeiten nicht von Bedeutung.

${ }^{\text {e} W a ̈ r e ~ d i e ~ G r e n z f l a ̈ c h e n k a p a z i t a ̈ t ~ n i c h t ~ p a r a l l e l ~ z u m ~ i s o l i e r e n d e n ~ W i d e r s t a n d ~ g e s c h a l t e t, ~ s o n d e r n ~ w u ̈ r d e ~}$ diesen ersetzen, könnte man das gewohnte Gleichstromverhalten nicht reproduzieren. Es muss daher eine reale, mit einem Leckwiderstand überbrückte Kapazität verwendet werden.
} 
mit der hier gewählten Umsetzung erreicht man jedoch einen realistischeren Beitrag der größeren relativen Oberfläche kleiner Domänen und stark gekrümmter Domänenwände.

Um das Temperaturverhalten studieren zu können, wurden jetzt einerseits die metallischen Widerstände mit einer quadratischen Temperaturabhängigkeit $R_{\mathrm{M}}=(T / 15)^{2}$ und die isolierenden mit einer thermisch aktivierten Leitfähigkeit $R_{\mathrm{I}}=e^{1500 / T}$ versehen (Siehe Abb. A.3 (a)). Für den metallischen Volumenanteil $p$-also die Besetzungswahrscheinlichkeit einer Verbindung mit einem metallischen Element - wurde der in Abb. A.3 (b) gezeigte Verlauf gewählt. Alle eingebauten Kapazitäten haben den Wert $C=4 \times 10^{-9} \mathrm{f}$

(a) Widerstandsverläufe

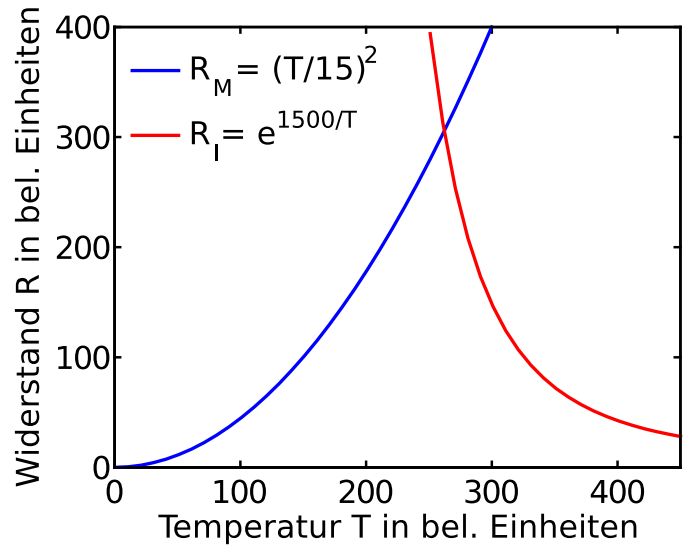

(b) Metallischer Volumenanteil

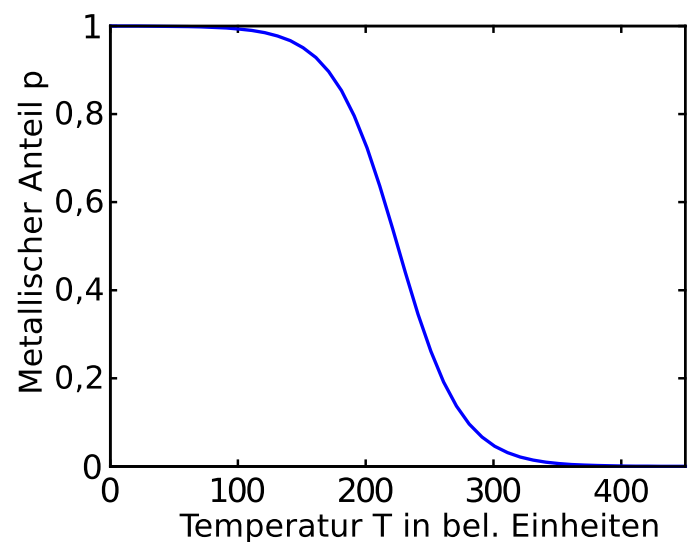

Abbildung A.3: Parameterwahl des Zufallsnetzwerks. (a) Widerstandsverlauf der metallischen und isolierenden Elemente. (b) Temperaturabhängigkeit des metallischen Anteils $p$ im Netzwerk.

In Abb. A.4 sind jetzt die für ein $80 \times 80$-Netzwerk errechneten Temperaturverläufe des Gesamtwiderstands bei verschiedenen Frequenzen dargestellt. ${ }^{g}$ Man erkennt ein qualitativ sehr ähnliches Frequenzverhalten wie in der zur Motivation angeführten Messung: Für niedrige Frequenzen spielen die zusätzlichen Kapazitäten keine Rolle und man erhält den typischen Metall-Isolator-Übergang des Gleichstromwiderstands. Mit steigender Frequenz flacht das Widerstandsmaximum am Übergang sukzessive ab und wird schließlich in ein Minimum verkehrt. Entfernt man sich vom Übergang, nimmt die Anzahl der ergänzten Kapazitäten ab, und die Hochfrequenzkurven nähern sich dem Gleichstromverlauf an.

Wie realistisch sind aber die untersuchten Perkolationsnetzwerke wirklich? Für verschiedene metallische Anteile $p$ sind in Abb. A.5 exemplarische Widerstandsnetzwerke dargestellt. Für kleine Konzentrationen $0<p \ll 0,5$ befinden sich nur wenige metallische Bereiche in einer isolierenden Matrix. Sie sind von einer Schale aus kapazitiven Elementen umgeben. Bei steigenden Frequenzen führt dies zu einer effektiven Vergrößerung der metallischen Inseln, die aber fernab der Perkolationsschwelle noch immer den kleineren Anteil des Stromes transportieren. Für große Konzentrationen $1>p \gg 0,5$ ergeben sich ebenfalls nur kleine

\footnotetext{
${ }^{\mathrm{f}}$ Der verwendete Parametersatz ist nicht für eine möglichst exakte Reproduktion der experimentellen Daten optimiert worden. Es soll lediglich ein qualitativer Eindruck vermittelt werden.

${ }^{\mathrm{g}}$ Zusätzlich wurde für jeden Temperatur- und Frequenzpunkt jeweils über fünf zufällige Netzwerke gemittelt.
} 


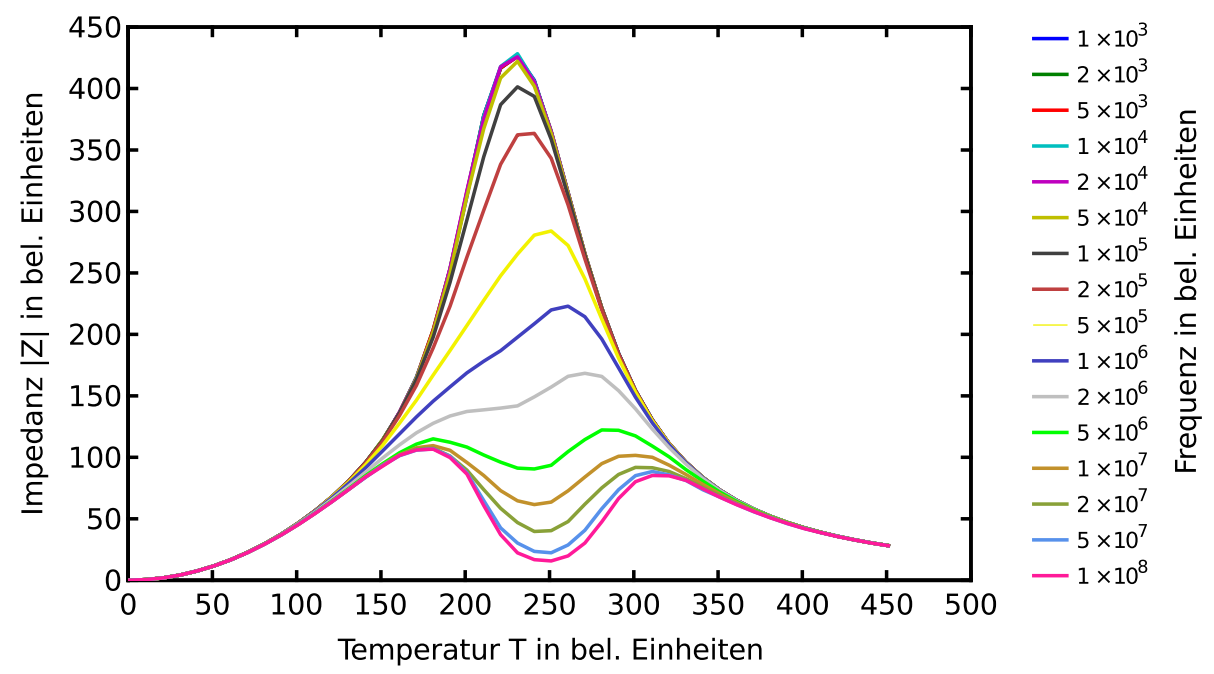

Abbildung A.4: Temperatur- und Frequenzabhängigkeit der Impedanz eines zufälligen $R C$-Netzwerkes. Für große Frequenzen ergibt sich zunächst eine Abplattung des Widerstandsmaximums, welches sich schließlich sogar in ein Minimum umkehrt.

Abweichungen: Die Pfade des ohnehin geschlossenen Perkolationsnetzwerkes werden noch etwas verbreitert. Die wenigen isolierenden Elemente grenzen bei einer wirklich zufälligen Besetzung in der Regel nicht aneinander, sodass praktisch alle Isolatoren mit einem Kondensator überbrückt werden. Der eigentlich erwünschte Aufbau, bei welchem nur die Randbereiche isolierender Regionen bei hohen Frequenzen kurzgeschlossen werden, wird also bei diesem Vorgehen nicht erreicht.

(a) Netzwerk für $\mathrm{p}=0,9$

(b) Netzwerk für $\mathrm{p}=\mathbf{0 , 5}$

(c) Netzwerk für $\mathrm{p}=0,1$
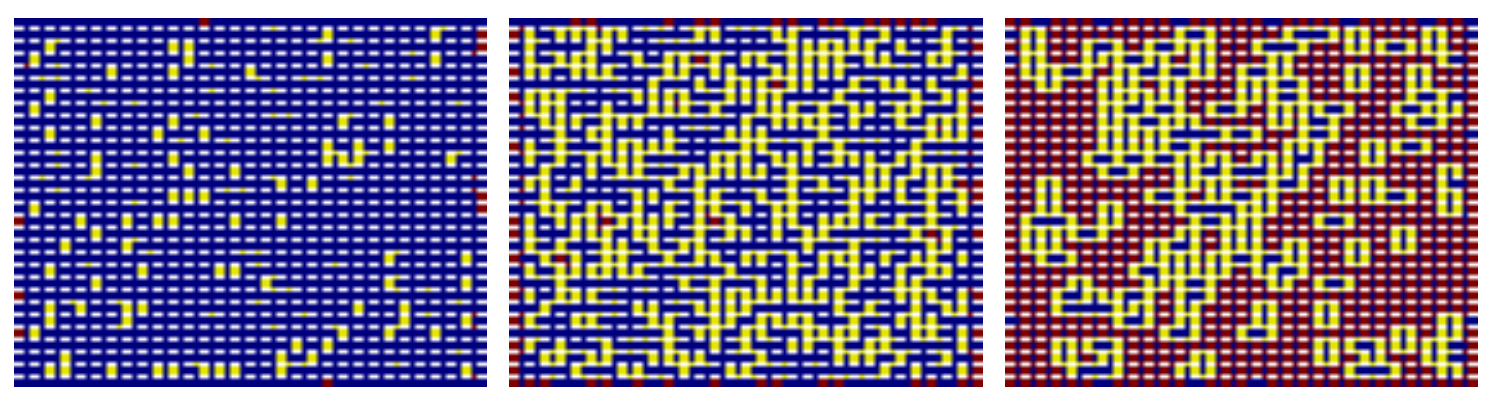

Abbildung A.5: Exemplarische $30 \times 30-R C-N e t z w e r k e$ für verschiedene metallische Anteile $p$. Metallische Elemente sind blau dargestellt, Isolatoren rot und kapazitiv überbrückte Isolatoren gelb. Bei großen (a) und kleinen (c) metallischen Anteilen entstehen kapazitiv überbrückte beziehungsweise umrandete Inseln der Minoritätsphase. Nahe der Perkolationsschwelle wird ein Großteil des Netzwerkes kapazitiv überbrückt (b).

Für mittlere Konzentrationen $p \approx 0,5$ ergibt sich zwar wie in den Messdaten mit steigender Frequenz ein drastischer Einbruch im Widerstand. Das Widerstandsnetzwerk erscheint jedoch umso unrealistischer, je näher man dem Übergang kommt (Vergleiche Abb. A.5 (b)). 
Weil durch die Zufallsbesetzung die überwiegende Mehrheit der isolierenden Widerstände mindestens einen metallischen Nachbarn hat, werden praktisch alle Isolatoren mit Kapazitäten überbrückt. Dieses einfache Modell erscheint also an der Perkolationsschwelle stark übertrieben. Der an sich schon vielversprechende Ansatz muss demnach insbesondere in der Nähe der Übergänge mit einem wirklichkeitsnahen Abbild der mikroskopischen Phasenseparation kombiniert werden, welches ausgedehnte „Cluster" aus isolierenden Elementen enthält, die von einer dünnen kapazitiven Schicht umrandet sind. Für das im folgenden Abschnitt verwendete RFIM ergibt sich durch die ferromagnetische Kopplung eine Grenzflächenenergie, wodurch die Ausbildung sehr kleiner Cluster ungünstiger wird.

\section{A.4. Random-Field Ising Modell}

Das RFIM ${ }^{[69]}$ wurde bereits sehr erfolgreich zur Simulation perkolativer Transportphänomene in Manganaten eingesetzt. Wie schon in Abschnitt 1.5 dargestellt, zeigten Moreo et al. mit Hilfe dieses Modells, wie sich aus einer kleinskaligen Unordnung eine ausgedehnte Phasenseparation ergeben kann. ${ }^{[68]}$ Burgy et al. nutzten die Möglichkeit, durch Monte-Carlo-Simulationen verwandter Modelle Netzwerke mit einer realistisch anmutenden räumlichen Phasenkonfiguration zu erstellen, um perkolative Widerstandsphänomene zu betrachten. ${ }^{[160]}$ Wir wollen diesen Ansatz aufgreifen, um nahe der Perkolationsschwelle die oben dargestellten Unzulänglichkeiten des reinen Zufallsnetzwerkes bei kapazitiver Ergänzung zu umgehen. ${ }^{\text {h }}$ Wir untersuchen also den bereits in Gleichung 1.5 gegebenen Hamilton-Operator:

$$
\mathcal{H}=-J \sum_{\langle i, j\rangle} S_{i}^{z} S_{j}^{z}-\sum_{i} h_{i} S_{i}^{z} \quad \text { mit } \quad S_{i}^{z} \in\{-1,+1\} .
$$

$J$ vermittelt eine ferromagnetische Kopplung der $z$-Komponenten $S_{i}^{z}$ benachbarter Spins; die Unordnung wird dem System hier durch ein lokales zufälliges Magnetfeld $h_{i}$ aufgeprägt. Die Domänenkonfiguration und damit das Perkolationsverhalten ist ferner von der Temperatur abhängig; für eine vorgegebene Temperatur wird jetzt eine Monte-Carlo-Simulation durchgeführt; der Einfachheit halber wurde hierfür ein leicht modifizierter MetropolisAlgorithmus implementiert. ${ }^{[167,168]}$

Wir konstruieren zunächst ein zweiphasiges Widerstandsnetzwerk, in welches für die auf- und abwärtsgerichteten Spins metallische beziehungsweise isolierende Widerstände eingefügt werden. Danach erfolgt wieder eine Suche nach Phasengrenzen, an welchen die äußerste isolierende Schicht durch kapazitive Elemente ergänzt wird. Bei ausreichend kleiner Unordnung entsteht so eine Phasenseparation des Widerstands auf großen Längenskalen - einerseits in Bezug auf den Abstand der Gitterpunkte, damit einhergehend aber auch auf die Breite der kapazitiven Grenzschicht. Je kleiner also die Unordnung gewählt wird, desto geringer ist die relative Dicke der kapazitiven Grenzschicht. Im Limes sehr starker Unordnung oder großer Temperaturen erhält man wieder ein reines Zufallsnetzwerk.

\footnotetext{
${ }^{\mathrm{h}}$ Die Verwendung des RFIM ist nur bei der kritischen Dimensionalität $d=2$ sinnvoll - im dreidimensionalen Falle sind unphysikalisch starke Zufallsfelder nötig, um einen raumfüllenden ferromagnetisch geordneten Zustand zu destabilisieren. Für eine Erweiterung auf drei Dimensionen muss man ein komplexeres Modell zugrundelegen. ${ }^{[161]}$
} 
Weil umfangreiche Untersuchungen für verschiedene Parametersätze noch ausstehen, beschränken wir uns hier auf das in Abb. A.6 (a) exemplarisch gezeigte Ergebnis einer Monte-Carlo-Simulation für ein $200 \times 200$-Netzwerk. Es wurden eine Kopplungsstärke $J=1$, eine Temperatur $T=0,4$ und ein Zufallsfeld $h_{i} \in[-3,+3]$ vorgegeben. Für die Übertragung auf das $R C$-Netzwerk (Abb. A.6 (b)) wurden die Widerstände $R_{\mathrm{M}}=1$ und $R_{\mathrm{I}}=1000$ sowie eine Grenzflächenkapazität $C=4 \times 10^{-9}$ verwendet. In Abb. A.7 ist die resultierende Frequenzabhängigkeit der Impedanz aufgetragen.

(a) Monte-Carlo-Simulation

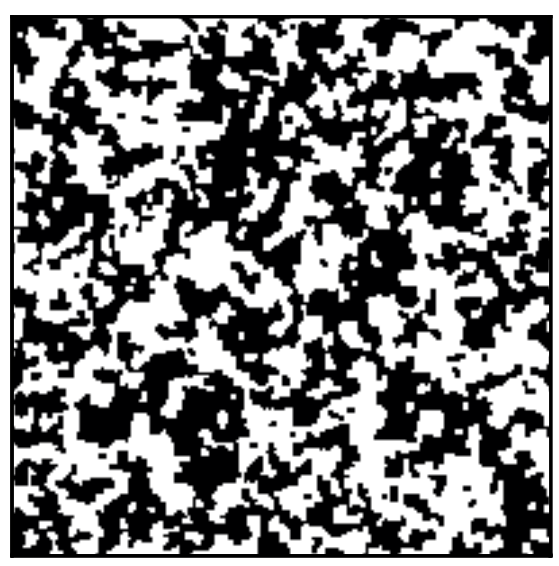

(b) RC-Netzwerk

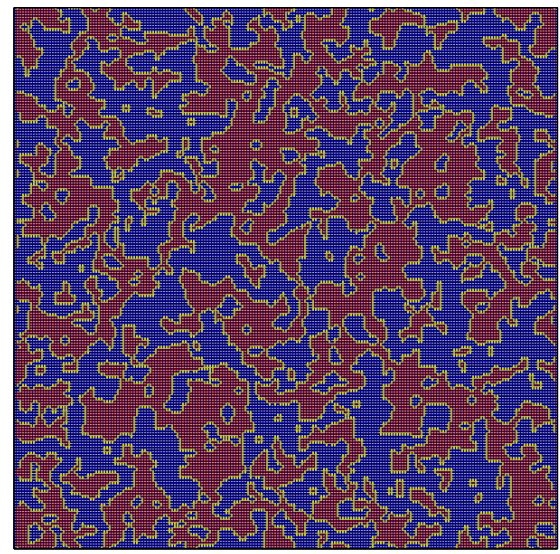

Abbildung A.6: Random-Field Ising Modell mit $J=1, T=0,4$ und $h_{i} \in[-3,+3]$. (a) Ergebnis einer Monte-Carlo-Simulation. (b) Übertragung auf ein $R C$-Netzwerk mit metallischen Widerständen (blau), isolierenden Elementen (rot) und Grenzflächenkapazitäten (gelb).

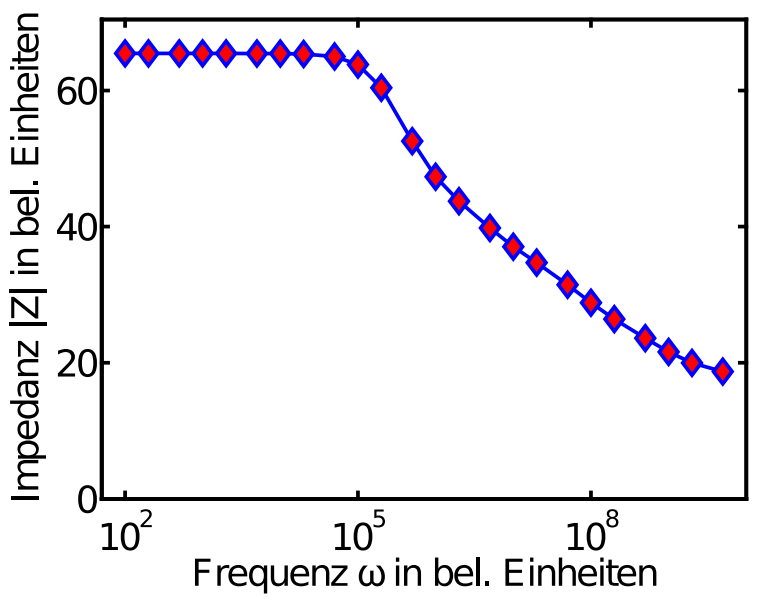

Abbildung A.7: Frequenzabhängiger Impedanzverlauf des $R C$-Netzwerkes. Mit steigender Frequenz folgt einem quasikonstanten Plateau nach einem abrupten Abknicken eine starke Widerstandsabnahme. 
Die aus der Knotenpotentialanalyse bekannten Spannungsabfälle zwischen den Knoten erlauben eine direkte Bestimmung des Stromflusses durch die einzelnen Netzwerkkomponenten. Diese sind in Abb. A.8 für drei ausgewählte Frequenzen als Farbkarten visualisiert; es ist der Nettostrom über die einzelnen Knoten farbkodiert dargestellt. Für tiefe Frequenzen beobachtet man das übliche Perkolationsverhalten: Nur wenige Strompfade tragen signifikant zum Transport bei, der Gesamtwiderstand wird durch sogenannte Hot Spots dominiert - Engstellen im Perkolationspfad, wo die Stromdichten besonders groß sind.

Unter sukzessiver Erhöhung der Frequenz tragen die Grenzflächenkapazitäten mehr und mehr zum Transport bei. Dabei bewirken sie in erster Linie eine Verbreiterung des Strompfades, welche vor allem an den hot spots den Stromfluss deutlich erleichtert. Zusätzlich werden neue Pfade ,angeschlossen“: Dort wo nur eine dünne isolierende Barriere zwei leitende Teilstücke trennt, eröffnet sich bei ausreichend großer Frequenz ein neuer stromführender Zweig.

Bei sehr großen Frequenzen wird schließlich die Grenzflächenkapazität leitfähiger als die metallische Phase selbst. Der Transport findet dann vornehmlich entlang der Grenzflächen statt. Ob diese Situation tatsächlich physikalisch sinnvoll ist, erscheint fraglich. Die deutliche Abnahme der Impedanz setzt aber ja schon bei der kapazitiven Verbreiterung für deutlich kleinere Frequenzen ein. Zumindest dieses Szenario erscheint durchaus realistisch.

Das RFIM erlaubt also, den im reinen Zufallsnetzwerk schwer zu behandelnden Fall einer Phasenbalance von $p \approx 0,5 \mathrm{zu}$ untersuchen. Man erhält auch mit einem (a) Strompfade für $\omega=5 \times 10^{5}$

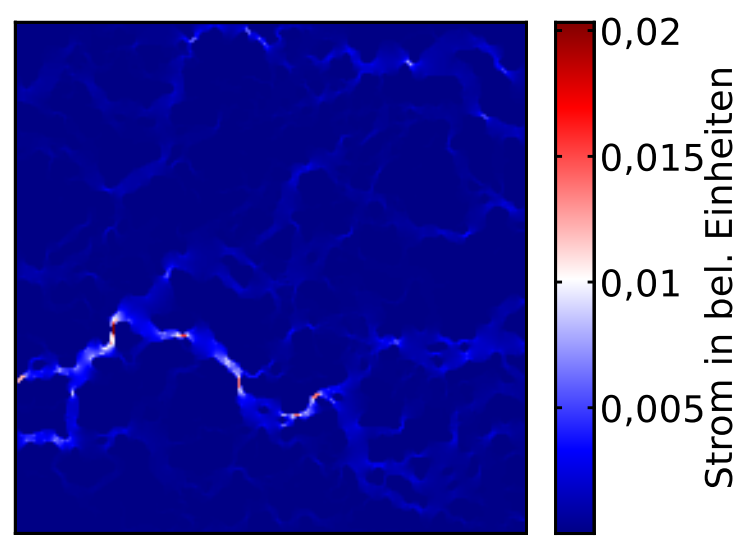

(b) Strompfade für $\omega=5 \times 10^{7}$

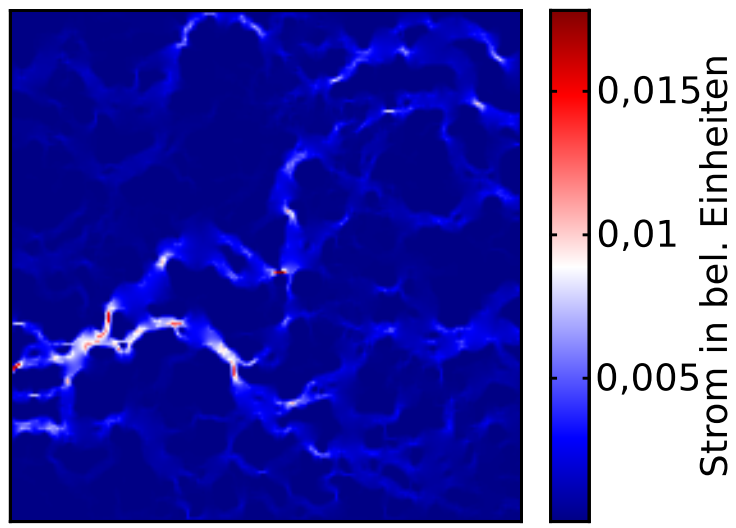

(c) Strompfade für $\omega=5 \times 10^{9}$

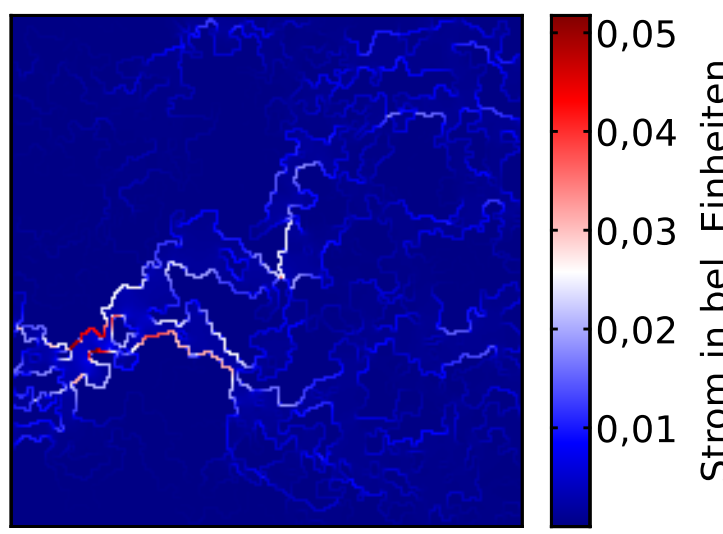

Abbildung A.8: Strompfade durch ein RFIMbasiertes $R C$-Netzwerk für verschiedene Frequenzen $\omega$. 
kleineren Anteil kapazitiver Elemente ein Verhalten, welches die Ausbildung des Widerstandsminimums in Abb. A.1 erklärlich macht.

\section{A.5. Fazit}

Das hier eingeführte Simulationsverfahren stellt einen ersten Versuch dar, AC-Transporteigenschaften phasenseparierter Systeme durch Perkolationseffekte in $R C$-Netzwerken zu beschreiben. Dabei steht nicht die Ergründung der jeweiligen mikroskopischen Natur der beteiligten Phasen im Vordergrund.

Es wurde gezeigt, dass die Simulation einfacher Zufallsnetzwerke unter Ergänzung kapazitiver Elemente bereits qualitative Übereinstimmungen mit sonst unverständlich erscheinenden Transportmessungen ergeben. Dem Problem eines unrealistisch erscheinenden Überbrückens fast der gesamten isolierenden Phase an der Perkolationsschwelle - sodass sich bei hohen Frequenzen ein Kurzschluss über einen kapazitiven Perkolationspfad ereignet - kann durch die Verwendung komplexerer Modelle begegnet werden. Anhand des RFIM wurde gezeigt, wie man auf einfache Weise eine Separation der Längenskalen der großen Domänenausbreitung gegenüber der kleinen Dicke einer Grenzschicht implementieren kann. Auch dieser Ansatz zeigt, dass sich bei hohen Frequenzen eine starke Impedanzabnahme ereignet.

Die vielversprechenden Ergebnisse erster Simulationsläufe sollten Anlass zu detaillierteren Studien geben. Dem AC-Transportverhalten könnte so zukünftig bei der Untersuchung intrinsisch phasenseparierter Systeme eine größere Bedeutung zuteil werden. Je nach Wahl des zugrundeliegenden Modells könnten so auch Magnetowiderstands- und kapazitätseffekte untersucht werden. Darüberhinaus ließen sich die dargestellten Modellierungen sicherlich auch zur Beschreibung polykristalliner Systeme anpassen. Proben mit permanenten, das heißt temperaturunabhängig vorliegenden Grenzflächen, wie zum Beispiel nanokolumnare Manganatschichten, sollten mit ähnlichen Ansätzen behandelt werden können. 


\section{Abbildungsverzeichnis}

1.1. Metall-Isolator-Übergang und CMR-Effekt. . . . . . . . . . . . . . . . . . . 4

1.2. Struktur perowskitischer Manganate (schematisch) . . . . . . . . . . 5

1.3. Modifikationen der idealen Perowskitstruktur. . . . . . . . . . . . . 6

1.4. 3d-Orbitale des Mangans . . . . . . . . . . . . . . . . . . . 7

1.5. Kristallfeldaufspaltung und Jahn-Teller-Effekt. . . . . . . . . . . . . . 7

1.6. Superaustausch . . . . . . . . . . . . . . . . . . . . . . . . . 9 9

1.7. Doppelaustausch . . . . . . . . . . . . . . . . . . . . . . . 10

1.8. Orbital- und Spinordnung vom CE-Typ (schematisch) . . . . . . . . . . . 11

1.9. Phasendiagramm von $\mathrm{La}_{1-\mathrm{x}} \mathrm{Ca}_{\mathrm{x}} \mathrm{MnO}_{3} \ldots \ldots \ldots \ldots$

1.10. Zufälliges Widerstandsnetzwerk mit perkolativem Widerstandsverhalten . . 15

1.11. Effektives Zweiwiderstandsmodell nach Mayr . . . . . . . . . . . . . . . 16

2.1. Unipolares und bipolares Schalten (schematisch). . . . . . . . . . . . 20

2.2. MIM-Schichtstruktur und Anwendung als RRAM. . . . . . . . . . . . . 22

3.1. Metallorganische Aerosoldeposition . . . . . . . . . . . . . . . . . 27

3.2. Funktionsweise eines Röntgendiffraktometers in Bragg-Brentano-Geometrie 28

3.3. Lennard-Jones-Potential und Kraft-Verschiebungs-Kurve. . . . . . . . . . . 32

3.4. Funktionsweise der C-AFM-Methode . . . . . . . . . . . . . . . . . 34

3.5. Aufbau des Omicron VT-AFM . . . . . . . . . . . . . . . . 36

4.1. Röntgendiffraktogramm der $\mathrm{La}_{0,8} \mathrm{Ca}_{0,2} \mathrm{MnO}_{3}: \mathrm{MgO}$-Probe . . . . . . . . . . 42

4.2. Makroskopische elektrische Eigenschaften: $R(T)$-Verlauf mit und ohne Magnetfeld, ausgewählte $I$ - $U$-Kennlinien $\ldots \ldots \ldots$

4.3. STM-Topographiebild . . . . . . . . . . . . . . . . . . . 44

4.4. Höhenprofil zu Abb. $4.3 \ldots \ldots \ldots \ldots$. . . . . . . . . . . . . 45

4.5. Widerstandsschalten: Gemittelte $I$ - $U$-Kennlinien $\ldots \ldots \ldots \ldots$. . . . . . . 47

4.6. Wiederholtes Schalten, Ermattung mit fortschreitender Pulszahl . . . . . . . 48 
4.7. Stromkarte und Topographie: Linienförmige, metallisch geschaltete Bereiche

4.8. Stromkarte und Topographie: Korrelation zwischen der Morphologie und der Ausdehnung einer entstandenen metallischen Region . . . . . . . . . . 50

4.9. Stromkarte und Topographie: Herzförmiges Muster und Satellitenflecken . . 51

4.10. Stromkarten: Isolierende Bereiche in metallischer Umgebung, metallisch geschaltete Region für $T<T_{\mathrm{MI}} \ldots \ldots \ldots \ldots$. . . . . . . . 52

4.11. Oberflächenmodifikationen durch einen Spannungspuls . . . . . . . . . . 53

4.12. Räumliche Ausdehnung metallischer Flecken in Abhängigkeit der Pulsstärken und -intervalle . . . . . . . . . . . . . . . . . . . 54

4.13. Abhängigkeit des äquivalenten Radius metallischer Domänen von der Pulsamplitude und -dauer (zu Abb. 4.12) . . . . . . . . . . . . 55

4.14. Topographie-Entwicklung zu Abb. 4.12 . . . . . . . . . . . . 56

4.15. Rückbildung einer metallischen Domäne: Stromkarten . . . . . . . . . . . 58

4.16. Rückbildung einer metallischen Domäne: Zeitabhängigkeit des äquivalenten Radius . . . . . . . . . . . . . . . . . . . 59

4.17. Pulsserienexperimente: Schematische Pulsfolge und exemplarische Stromver-

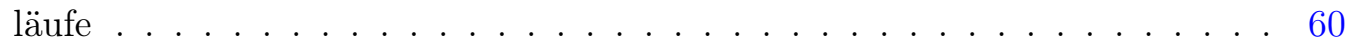

4.18. Gemittelte Stromentwicklung im Verlauf von Pulsfolgen . . . . . . . . . . 61

4.19. Stromkarte einer LMO-terminierten LCMO-Schicht . . . . . . . . . . . . . 63

4.20. Topographie und $I$ - $U$-Charakteristik des LMO-terminierten Films . . . . . . 64

4.21. Diskrete Stromwerte auf einer LSMO-Oberfläche . . . . . . . . . . . . . 65

4.22. Topographie zur Stromkarte Abb. 4.21 . . . . . . . . . . . . . . . 65

4.23. Histogramm zu Abb. $4.21 \ldots \ldots$. . . . . . . . . . . . 67

4.24. Korrelation zwischen lokaler Leitfähigkeit und Höhenprofil . . . . . . . . . . 67

5.1. Magnetisches und strukturelles Phasendiagramm von Eisen. . . . . . . . . . 73

5.2. Kontaktfläche an der Oberfläche und Domänenwand. . . . . . . . . . . . . . 75

5.3. Zweiniveaumodell der Widerstandszustände. . . . . . . . . . . . . . . . . . 77

5.4. Modell eines strukturellen Übergangs. . . . . . . . . . . . . . . . . . . . . 80

5.5. Ausbreitung der metallischen Domäne durch den Feldgradienten an der Domänenwand . . . . . . . . . . . . . . . . 81

5.6. Zylinderschalenmodell der Feldgeometrie . . . . . . . . . . . . . . . . . 91

5.7. Domänenwachstum gemäß Kriechformel . . . . . . . . . . . . . . . . . 95

5.8. Hypothetische Domänenentwicklung im Verlauf von Pulsfolgen. . . . . . . . 96

5.9. Diskrete Leitfähigkeitsplateaus als Folge monolagenweisen Umschaltens . . . 100

A.1. AC-Transportverhalten einer $\left(\mathrm{La}_{0,6} \mathrm{Pr}_{0,4}\right)_{0,7} \mathrm{Ca}_{0,3} \mathrm{MnO}$-Schicht . . . . . . . . 108

A.2. Kontruktionsprinzip der $R C$-Netzwerke . . . . . . . . . . . . . . . . 110

A.3. Parameterwahl des Zufallsnetzwerks . . . . . . . . . . . . . . . . . . 111

A.4. Temperatur- und Frequenzabhängigkeit der Impedanz eines zufälligen $R C$ -

Netzwerkes . . . . . . . . . . . . . . . . . . . . . 112

A.5. RC-Netzwerke für verschiedene metallische Anteile $p \ldots \ldots$. . . . . . . . 112

A.6. Monte-Carlo-Simulation eines RFIM und Übertragung auf ein $R C$-Netzwerk 114

A.7. Frequenzabhängiger Impedanzverlauf . . . . . . . . . . . . . . . . . . . . 114

A.8. Strompfade im RFIM-basierten $R C$-Netzwerk . . . . . . . . . . . . 115 


\section{Literatur}

1. R. von Helmolt, J. Wecker, B. Holzapfel, L. Schultz \& K. Samwer: Giant negative magnetoresistance in perovskitelike $\mathrm{La}_{2 / 3} \mathrm{Ba}_{1 / 3} \mathrm{MnO}_{\mathrm{x}}$ ferromagnetic films. Physical Review Letters 71, 2331 - 2333 (1993).

2. J. H. Park, E. Vescovo, H. J. Kim, C. Kwon, R. Ramesh \& T. Venkatesan: Direct evidence for a half-metallic ferromagnet. Nature 392, $794-796$ (1998).

3. A. Asamitsu, Y. Tomioka, H. Kuwahara \& Y. Tokura: Current switching of resistive states in magnetoresistive manganites. Nature 388, 50 - 52 (1997).

4. L. CHuA: Memristor - The missing circuit element. IEEE Transactions on Circuit Theory 18, 507 - 519 (1971).

5. L. Chua \& S. M. Kang: Memristive devices and systems. Proceedings of the IEEE 64, $209-223$ (1976).

6. R. Waser \& M. Aono: Nanoionics-based resistive switching memories. Nature Materials 6, $833-840$ (2007).

7. A. SAwA: Resistive switching in transition metal oxides. Materials Today 11, $28-36$ (2008).

8. R. Waser, R. Dittmann, G. Staikov \& K. Szot: Redox-Based Resistive Switching Memories - Nanoionic Mechanisms, Prospects, and Challenges. Advanced Materials 21, $2632-2663$ (2009).

9. X. Chen, N. Wu, J. Strozier \& A. Ignatiev: Spatially extended nature of resistive switching in perovskite oxide thin films. Applied Physics Letters 89, 063507 (2006).

10. M. Mayr, A. Moreo, J. A. Vergés, J. Arispe, A. Feiguin \& E. Dagotto: Resistivity of Mixed-Phase Manganites. Physical Review Letters 86, 135 - 138 (2001).

11. J. Burgy, M. Mayr, V. Martin-Mayor, A. Moreo \& E. Dagotto: Colossal Effects in Transition Metal Oxides Caused by Intrinsic Inhomogeneities. Physical Review Letters 87, 277202 (2001). 
12. G. H. Jonker \& J. H. VAn SAnten: Ferromagnetic compounds of manganese with perovskite structure. Physica 16, 337 - 349 (1950).

13. J. H. VAN SANTEN \& G. H. JONKER: Electrical conductivity of ferromagnetic compounds of manganese with perovskite structure. Physica 16, 599 - 600 (1950).

14. E. O. Wollan \& W. C. Koehler: Neutron Diffraction Study of the Magnetic Properties of the Series of Perovskite-Type Compounds $[(1-\mathrm{x}) \mathrm{La}, \mathrm{xCa}] \mathrm{MnO}_{3}$. Physical Review 100, 545 - 563 (1955).

15. J. Volger: Further experimental investigations on some ferromagnetic oxidic compounds of manganese with perovskite structure. Physica 20, 49 - 66 (1954).

16. C. W. Searle \& S. T. Wang: Studies of the ionic ferromagnet $(\mathrm{LaPb}) \mathrm{MnO}_{3}$ III. Ferromagnetic resonance studies. Canadian Journal of Physics 47, $2703-2708$ (1969).

17. C. W. Searle \& S. T. Wang: Studies of the ionic ferromagnet $(\mathrm{LaPb}) \mathrm{MnO}_{3}$. V. Electric transport and ferromagnetic properties. Canadian Journal of Physics 48, 2023 - 2031 (1970).

18. K. Chahara, T. Ohno, M. Kasai \& Y. Kozono: Magnetoresistance in magnetic manganese oxide with intrinsic antiferromagnetic spin structure. Applied Physics Letters 63, 1990 - 1992 (1993).

19. R. Kusters, J. Singleton, D. Keen, R. McGreevy \& W. Hayes: Magnetoresistance measurements on the magnetic semiconductor $\mathrm{Nd}_{0.5} \mathrm{~Pb}_{0.5} \mathrm{MnO}_{3}$. Physica B: Condensed Matter 155, 362 - 365 (1989).

20. S. Jin, T. H. Tiefel, M. McCormack, R. A. Fastnacht, R. Ramesh \& L. H. Chen: Thousandfold Change in Resistivity in Magnetoresistive La-Ca-Mn-O Films. Science 264, 413 - 415 (1994).

21. E. Dagotto: Nanoscale Phase Separation and Colossal Magnetoresistance (SpringerVerlag, 2003).

22. Colossal magneto-resistive oxides. Hrsg. Y. TokURA (Gordon und Breach Science Publishers, 2000).

23. J. M. D. Coey, M. Viret \& S. von MolnáR: Mixed-valence manganites. Advances in Physics 48, 167 - 293 (1999).

24. M. B. SAlamon \& M. Jaime: The physics of manganites: Structure and transport. Reviews of Modern Physics 73, 583 - 628 (2001).

25. E. Dagotto, T. Hotta \& A. Moreo: Colossal magnetoresistant materials: the key role of phase separation. Physics Reports 344, 1 - 153 (2001).

26. E. Dagotto: Open questions in CMR manganites, relevance of clustered states and analogies with other compounds including the cuprates. New Journal of Physics 7, 67 (2005).

27. V. Moshnyaga: Metal-Insulator Transition and Magnetoresistance in Manganite Thin Films: Lattice Strain and Disorder Effects. In: Frontiers in Magnetic Materials (Hrsg. A. V. NARLikAR), 415 - 458 (Springer, 2005). 
28. V. Moshnyaga \& K. Samwer: Ferromagnetic Manganite Films. In: Handbook of Magnetism and Advanced Magnetic Materials (Hrsg. H. Kronmüller \& S. PARKIN), 2727 - 2755 (John Wiley \& Sons, 2007).

29. L. Sudheendra, V. Moshnyaga \& K. Samwer: Metal-insulator transition and colossal magnetoresistance: relevance of electron-lattice coupling and electronic phase separation. Contemporary Physics 48, 349 - 364 (2007).

30. Y. TOKURA: Critical features of colossal magnetoresistive manganites. Reports on Progress in Physics 69, 797 (2006).

31. L. M. Rodriguez-Martinez \& J. P. Attfield: Cation disorder and size effects in magnetoresistive manganese oxide perovskites. Physical Review B 54, R15622 R15625 (1996).

32. V. Moshnyaga, L. Sudheendra, O. I. Lebedev, S. A. Köster, K. Gehrke, O. Shapoval, A. Belenchuk, B. Damaschke, G. van Tendeloo \& K. Samwer: A-Site Ordering versus Electronic Inhomogeneity in Colossally Magnetoresistive Manganite Films. Physical Review Letters 97, 107205 (2006).

33. S. Blundell: Magnetism in Condensed Matter (Oxford University Press, 2001).

34. Y. Tokura: Fundamental Features of Colossal Magnetoresistive Oxides. In: Colossal magneto-resistive oxides (Hrsg. Y. TokURA), 1 - 52 (Gordon und Breach Science Publishers, 2000).

35. H. A. Jahn \& E. Teller: Stability of Polyatomic Molecules in Degenerate Electronic States. I. Orbital Degeneracy. Proceedings of the Royal Society of London. Series A Mathematical and Physical Sciences 161, 220 - 235 (1937).

36. N. F. Mотт: Metal-Insulator Transitions, 2. Aufl. (Taylor \& Francis, 1990).

37. J. Zahanen, G. A. Sawatzky \& J. W. Allen: Band gaps and electronic structure of transition-metal compounds. Physical Review Letters 55, 418 - 421 (1985).

38. M. Imada, A. Fujimori \& Y. Tokura: Metal-insulator transitions. Reviews of Modern Physics 70, 1039 - 1263 (1998).

39. M. Opel: Magnetismus (Vorlesungsskript). WS 2004/2005, TU München (2005). $<$ http : / / www . wmi . badw . de/teaching/Lecturenotes / magnetismus / Kapitel 6.pdf $>$ (besucht am 30.10.2011).

40. C. Zener: Interaction Between the $d$ Shells in the Transition Metals. Physical Review 81, $440-444$ (1951).

41. C. Zener: Interaction between the $d$-Shells in the Transition Metals. II. Ferromagnetic Compounds of Manganese with Perovskite Structure. Physical Review 82, $403-405$ (1951).

42. P. W. Anderson \& H. Hasegawa: Considerations on Double Exchange. Physical Review 100, 675 - 681 (1955).

43. P. G. De Gennes: Effects of Double Exchange in Magnetic Crystals. Physical Review 118, $141-154(1960)$. 
44. T. Akimoto, Y. Maruyama, Y. Moritomo, A. Nakamura, K. Hirota, K. Ohoyama \& M. Ohashi: Antiferromagnetic metallic state in doped manganites. Physical Review B 57, R5594 - R5597 (1998).

45. F. Damay, A. Maignan, C. Martin \& B. Raveau: Cation size-temperature phase diagram of the manganites $\operatorname{Ln}_{0.5} \mathrm{Sr}_{0.5} \mathrm{MnO}_{3}$. Journal of Applied Physics 81, 1372 - 1377 (1997).

46. G. Zhao, K. Conder, H. Keller \& K. A. Muller: Giant oxygen isotope shift in the magnetoresistive perovskite $\mathrm{La}_{1-\mathrm{x}} \mathrm{Ca}_{\mathrm{x}} \mathrm{MnO}_{3+\mathrm{y}}$. Nature 381, 676 - 678 (1996).

47. A. J. Millis, P. B. Littlewood \& B. I. Shraiman: Double Exchange Alone Does Not Explain the Resistivity of $\mathrm{La}_{1-\mathrm{x}} \mathrm{Sr}_{\mathrm{x}} \mathrm{MnO}_{3}$. Physical Review Letters 74, $5144-5147$ (1995).

48. A. J. Millis, B. I. Shraiman \& R. Mueller: Dynamic Jahn-Teller Effect and Colossal Magnetoresistance in $\mathrm{La}_{1-\mathrm{x}} \mathrm{Sr}_{\mathrm{x}} \mathrm{MnO}_{3}$. Physical Review Letters 77, $175-178$ (1996).

49. A. J. Millis: Lattice effects in magnetoresistive manganese perovskites. Nature 392, $147-150$ (1998).

50. T. Holstein: Studies of polaron motion: Part I. The molecular-crystal model. Annals of Physics 8, 325 - 342 (1959).

51. T. Holstein: Studies of polaron motion: Part II. The "small" polaron. Annals of Physics 8, 343 - 389 (1959).

52. C. Hartinger, F. Mayr, A. Loidl \& T. Kopp: Polaronic excitations in colossal magnetoresistance manganite films. Physical Review B 73, 024408 (2006).

53. M. Viret, L. Ranno \& J. M. D. Coey: Magnetic localization in mixed-valence manganites. Physical Review B 55, 8067 - 8070 (1997).

54. V. Kiryukhin, T. Y. Koo, H. Ishibashi, J. P. Hill \& S.-W. Cheong: Average lattice symmetry and nanoscale structural correlations in magnetoresistive manganites. Physical Review B 67, 064421 (2003).

55. P. Dai, J. A. Fernandez-Baca, N. Wakabayashi, E. W. Plummer, Y. ToMIOKA \& Y. TOKURA: Short-Range Polaron Correlations in the Ferromagnetic $\mathrm{La}_{1-\mathrm{x}} \mathrm{Ca}_{\mathrm{x}} \mathrm{MnO}_{3}$. Physical Review Letters 85, 2553 - 2556 (2000).

56. C. Jooss, L. Wu, T. Beetz, R. F. Klie, M. Beleggia, M. A. Schofield, S. Schramm, J. Hoffmann \& Y. Zhu: Polaron melting and ordering as key mechanisms for colossal resistance effects in manganites. Proceedings of the National Academy of Sciences 104, 13597 - 13602 (2007).

57. L. Sudheendra, V. Moshnyaga, E. D. Mishina, B. Damaschke, T. Rasing $\&$ K. SAmWER: Direct imaging of lattice-strain-induced stripe phases in an optimally doped manganite film. Physical Review B 75, 172407 (2007).

58. A. S. Alexandrov \& A. M. Bratkovsky: Carrier Density Collapse and Colossal Magnetoresistance in Doped Manganites. Physical Review Letters 82, 141 - 144 (1999). 
59. A. S. Alexandrov, A. M. Bratkovsky \& V. V. Kabanov: Phase Coexistence and Resistivity near the Ferromagnetic Transition of Manganites. Physical Review Letters 96, 117003 (2006).

60. I. Leonov, N. Binggeli, D. Korotin, V. I. Anisimov, N. Stoji Ć \& D. VollHARDT: Structural Relaxation due to Electronic Correlations in the Paramagnetic Insulator $\mathrm{KCuF}_{3}$. Physical Review Letters 101, 096405 (2008).

61. I. Leonov, D. Korotin, N. BingGeli, V. I. Anisimov \& D. Vollhardt: Computation of correlation-induced atomic displacements and structural transformations in paramagnetic $\mathrm{KCuF}_{3}$ and $\mathrm{LaMnO}_{3}$. Physical Review B 81, 075109 (2010).

62. A. Urushibara, Y. Moritomo, T. Arima, A. Asamitsu, G. Kido \& Y. TOKURA: Insulator-metal transition and giant magnetoresistance in $\mathrm{La}_{1-\mathrm{x}} \mathrm{Sr}_{\mathrm{x}} \mathrm{MnO}_{3}$. Physical Review B 51, 14103 - 14109 (1995).

63. J. Hemberger, A. Krimmel, T. Kurz, H.-A. Krug von Nidda, V. Y. Ivanov, A. A. Mukhin, A. M. Balbashov \& A. Loidl: Structural, magnetic, and electrical properties of single-crystalline $\mathrm{La}_{1-\mathrm{x}} \mathrm{Sr}_{\mathrm{x}} \mathrm{MnO}_{3}(0.4<\mathrm{x}<0.85)$. Physical Review $B$ 66, 094410 (2002).

64. S. W. CheOng \& H. Y. Hwang: Ferromagnetism versus Charge/Orbital Ordering in Mixed-Valent Manganites. In: Colossal magneto-resistive oxides (Hrsg. Y. TOKURA), 237 - 280 (Gordon und Breach Science Publishers, 2000).

65. M. Uehara, S. Mori, C. H. Chen \& S. W. Cheong: Percolative phase separation underlies colossal magnetoresistance in mixed-valent manganites. Nature 399, $560-$ 563 (1999).

66. M. Fäth, S. Freisem, A. A. Menovsky, Y. Tomioka, J. Aarts \& J. A. Mydosh: Spatially Inhomogeneous Metal-Insulator Transition in Doped Manganites. Science 285, 1540 - 1542 (1999).

67. T. Becker, C. Streng, Y. Luo, V. Moshnyaga, B. Damaschke, N. Shannon \& K. SAmwer: Intrinsic Inhomogeneities in Manganite Thin Films Investigated with Scanning Tunneling Spectroscopy. Physical Review Letters 89, 237203 (2002).

68. A. Moreo, M. Mayr, A. Feiguin, S. Yunoki \& E. Dagotto: Giant Cluster Coexistence in Doped Manganites and Other Compounds. Physical Review Letters 84, 5568 - $5571(2000)$.

69. Y. IMRY \& S.-K. MA: Random-Field Instability of the Ordered State of Continuous Symmetry. Physical Review Letters 35, 1399 - 1401 (1975).

70. C. Renner, G. Aeppli, B. G. Kim, Y.-A. Soh \& S. W. Cheong: Atomic-scale images of charge ordering in a mixed-valence manganite. Nature 416, $518-521$ (2002).

71. S. Rössler, B. Padmanabhan, S. Elizabeth, H. L. Bhat, F. Steglich \& S. WIRTH: Atomically resolved scanning tunneling microscopy on perovskite manganite single crystals. Applied Physics Letters 96, 202512 (2010). 
72. R. P. Borges, W. Guichard, J. G. Lunney, J. M. D. Coey \& F. Ott: Magnetic and electric "dead" layers in $\left(\mathrm{La}_{0.7} \mathrm{Sr}_{0.3}\right) \mathrm{MnO}_{3}$ thin films. 89, $3868-3873$ (2001).

73. M. Bibes, L. Balcells, S. Valencia, J. Fontcuberta, M. Wojcik, E. JedryKA \& S. NADOLSKI: Nanoscale Multiphase Separation at $\mathrm{La}_{2 / 3} \mathrm{Ca}_{1 / 3} \mathrm{MnO}_{3} / \mathrm{SrTiO}_{3}$ Interfaces. Physical Review Letters 87, 067210 (2001).

74. M. J. Calderón, L. Brey \& F. Guinea: Surface electronic structure and magnetic properties of doped manganites. Physical Review B 60, 6698 - 6704 (1999).

75. A. Tebano, C. Aruta, S. Sanna, P. G. Medaglia, G. Balestrino, A. A. Sidorenko, R. De Renzi, G. Ghiringhelli, L. Braicovich, V. Bisogni \& N. B. Brookes: Evidence of Orbital Reconstruction at Interfaces in Ultrathin $\mathrm{La}_{0.67} \mathrm{Sr}_{0.33} \mathrm{MnO}_{3}$ Films. Physical Review Letters 100, 137401 (2008).

76. L. BREY: Electronic phase separation in manganite-insulator interfaces. Physical Review B 75, 104423 (2007).

77. K. Gehrke, V. Moshnyaga, K. Samwer, O. I. Lebedev, J. Verbeeck, D. Kirilenko \& G. VAn Tendeloo: Interface controlled electronic variations in correlated heterostructures. Physical Review B 82, 113101 (2010).

78. K. GeHrke: Magnetoelektrische Eigenschaften von Manganat-Titanat-Übergittern. Diss. (Georg-August-Universität Göttingen, 2009).

79. X. Chen, J. Strozier, N. J. Wu, A. Ignatiev \& Y. B. Nian: A study of the symmetry properties and multi-state nature of perovskite oxide-based electrical pulse induced resistance-change devices. New Journal of Physics 8, 229 (2006).

80. A. T. Waterman: The Electrical Conductivity of Molybdenite. Physical Review 21, 540 - 549 (1923).

81. S. R. Ovshinsky: Reversible Electrical Switching Phenomena in Disordered Structures. Physical Review Letters 21, 1450 - 1453 (1968).

82. T. W. Hickмотт: Low-Frequency Negative Resistance in Thin Anodic Oxide Films. Journal of Applied Physics 33, 2669 - 2682 (1962).

83. J. G. Simmons \& R. R. Verderber: New Conduction and Reversible Memory Phenomena in Thin Insulating Films. Proceedings of the Royal Society of London. Series A - Mathematical and Physical Sciences 301, 77 - 102 (1967).

84. J. F. Gibbons \& W. E. Beadle: Switching properties of thin NiO films. Solid-State Electronics 7, 785 - 790 (1964).

85. H. BIEDERMAN: Metal-insulator-metal sandwich structures with anomalous properties. Vacuum 26, 513 - 523 (1976).

86. G. Dearnaley, A. M. Stoneham \& D. V. Morgan: Electrical phenomena in amorphous oxide films. Reports on Progress in Physics 33, 1129 (1970).

87. H. Pagnia \& N. Sotnik: Bistable switching in electroformed metal-insulator-metal devices. physica status solidi (a) 108, 11 - 65 (1988). 
88. A. Beck, J. G. Bednorz, C. Gerber, C. Rossel \& D. Widmer: Reproducible switching effect in thin oxide films for memory applications. Applied Physics Letters 77, 139 - 141 (2000)

89. L. Ma, S. Pyo, J. OuYAng, Q. Xu \& Y. YAng: Nonvolatile electrical bistability of organic/metal-nanocluster/organic system. Applied Physics Letters 82, 1419 - 1421 (2003).

90. R. Müller, R. Naulaerts, J. Billen, J. Genoe \& P. Heremans: CutCnQ resistive nonvolatile memories with a noble metal bottom electrode. Applied Physics Letters 90, 063503 (2007).

91. R. S. Potember, T. O. Poehler \& D. O. Cowan: Electrical switching and memory phenomena in Cu-TCNQ thin films. Applied Physics Letters 34, 405 - 407 (1979).

92. D. B. Strukov, G. S. Snider, D. R. Stewart \& R. S. Williams: The missing memristor found. Nature 453, 80 - 83 (2008).

93. G.-S. Park, X.-S. Li, D.-C. Kim, R.-J. Jung, M.-J. LeE \& S. SeO: Observation of electric-field induced $\mathrm{Ni}$ filament channels in polycrystalline $\mathrm{NiO}_{\mathrm{x}}$ film. 91, 222103 (2007).

94. K. Szot, R. Dittmann, W. Speier \& R. Waser: Nanoscale resistive switching in $\mathrm{SrTiO}_{3}$ thin films. physica status solidi (RRL) 1, R86 - R88 (2007).

95. R. Fors, S. I. Khartsev \& A. M. Grishin: Giant resistance switching in metalinsulator-manganite junctions: Evidence for Mott transition. Physical Review B 71, 045305 (2005).

96. A. Baikalov, Y. Q. Wang, B. Shen, B. Lorenz, S. Tsui, Y. Y. Sun, Y. Y. Xue \& C. W. Chu: Field-driven hysteretic and reversible resistive switch at the $\mathrm{Ag}-\mathrm{Pr}_{0.7} \mathrm{Ca}_{0.3} \mathrm{MnO}_{3}$ interface. Applied Physics Letters 83, 957 - 959 (2003).

97. S. Tsui, A. Baikalov, J. Cmaidalka, Y. Y. Sun, Y. Q. Wang, Y. Y. Xue, C. W. Chu, L. Chen \& A. J. Jacobson: Field-induced resistive switching in metal-oxide interfaces. Applied Physics Letters 85, 317 - 319 (2004).

98. H. L. Ju, J. Gopalakrishnan, J. L. Peng, Q. Li, G. C. Xiong, T. Venkatesan \& R. L. GReEne: Dependence of giant magnetoresistance on oxygen stoichiometry and magnetization in polycrystalline $\mathrm{La}_{0.67} \mathrm{Ba}_{0.33} \mathrm{MnO}_{z}$. Physical Review B 51, 6143 6146 (1995).

99. M. J. Rozenberg, I. H. Inoue \& M. J. SÁnchez: Nonvolatile Memory with Multilevel Switching: A Basic Model. Physical Review Letters 92, 178302 (2004).

100. M. Quintero, P. Levy, A. G. Leyva \& M. J. Rozenberg: Mechanism of Electric-Pulse-Induced Resistance Switching in Manganites. Physical Review Letters 98, 116601 (2007).

101. A. SaWa, T. Fujil, M. KaWAsaki \& Y. Tokura: Hysteretic current-voltage characteristics and resistance switching at a rectifying $\mathrm{Ti} / \mathrm{Pr}_{0.7} \mathrm{Ca}_{0.3} \mathrm{MnO}_{3}$ interface. Applied Physics Letters 85, 4073 - 4075 (2004). 
102. S. M. SzE: Semiconductor Devices - Physics and Technology, 2. Aufl. (John Wiley \& Sons, 2002).

103. T. Fujil, M. Kawasaki, A. Sawa, H. Akoh, Y. Kawazoe \& Y. Tokura: Hysteretic current-voltage characteristics and resistance switching at an epitaxial oxide Schottky junction $\mathrm{SrRuO}_{3} / \mathrm{SrTi}_{0.99} \mathrm{Nb}_{0.01} \mathrm{O}_{3}$. Applied Physics Letters 86, 012107 (2005).

104. T. Driscoll, H.-T. Kim, B.-G. Chae, M. Di Ventra \& D. N. Basov: Phasetransition driven memristive system. Applied Physics Letters 95, 043503 (2009).

105. S. T. Hsu, T. Li \& N. AwAYA: Resistance random access memory switching mechanism. Journal of Applied Physics 101, 024517 (2007).

106. C. Jooss, J. Hoffmann, J. Fladerer, M. Ehrhardt, T. Beetz, L. Wu \& Y. ZHU: Electric pulse induced resistance change effect in manganites due to polaron localization at the metal-oxide interfacial region. Physical Review B 77, 132409 (2008).

107. M. Esseling: Grenzflächeneffekte in Manganatschichten. Diss. (Georg-AugustUniversität Göttingen, 2007).

108. V. Moshnyaga, M. Esseling, L. Sudheendra, O. I. Lebedev, K. Gehrke, G. Van Tendeloo \& K. Samwer: Memristor Behaviour in Nano-Sized Vertical LSMO/LSMO Tunnel Junctions. ArXiv e-prints. arXiv:1002.0495 [cond-mat.mes-hall] (2010).

109. V. Moshnyaga, I. Khoroshun, A. Sidorenko, P. Petrenko, A. Weidinger, M. Zeitler, B. Rauschenbach, R. Tidecks \& K. Samwer: Preparation of rare-earth manganite-oxide thin films by metalorganic aerosol deposition technique. Applied Physics Letters 74, 2842 - 2844 (1999).

110. I. V. Khoroshun, E. V. Karyaev, V. T. Moshnyaga, G. A. Kiosse, M. A. Krachun, V. M. Zakosarenko \& V. Y. Davydov: Characteristics of epitaxial $\mathrm{Y}-\mathrm{Ba}-\mathrm{Cu}-\mathrm{O}$ thin films grown by aerosol MOCVD technique. Superconductor Science and Technology 3, 493 (1990).

111. Diffraktometer D 5000. Siemens Aktiengesellschaft (1989).

112. D8 Advance / Discover Röntgen-Diffraktometer. Bruker AXS GmbH (1999).

113. N. W. Ashcroft \& D. N. Mermin: Festkörperphysik, 3. Aufl. (Oldenbourg Wissenschaftsverlag GmbH, 2007).

114. A. SegmüLLER: Observation of X-ray interferences on thin films of amorphous silicon. Thin Solid Films 18, 287 - 294 (1973).

115. W. Hink \& W. Petzold: X-ray Interference Patterns of Vapor Deposited Aluminum Layers. Zeitschrift für angewandte Physik 10, 553 (1958).

116. Scanning Tunneling Microscopy I. Hrsg. H.-J. GÜntherodt \& R. Wiesendanger (Springer-Verlag, 1992).

117. Scanning Tunneling Microscopy II. Hrsg. R. Wiesendanger \& H.-J. GÜntherodt (Springer-Verlag, 1992). 
118. Scanning Tunneling Microscopy III. Hrsg. R. Wiesendanger \& H.-J. Güntherodt (Springer-Verlag, 1993).

119. E. Meyer, H. J. Hug \& R. Bennewitz: Scanning Probe Microscopy (SpringerVerlag, 2004).

120. B. Cappella \& G. Dietler: Force-distance curves by atomic force microscopy. Surface Science Reports 34, 1 - 104 (1999).

121. D. SARID: Scanning Force Microscopy (Oxford University Press, 1991).

122. The VT Beam Deflection AFM User's Guide. 1.0. Omicron NanoTechnology GmbH (Taunusstein, 2000).

123. C. C. Williams: Two-Dimensional Dopant Profiling by Scanning Capacitance Microscopy. Annual Review of Materials Science 29, 471 - 504 (1999).

124. J. M. R. Weaver \& D. W. Abraham: High resolution atomic force microscopy potentiometry. Journal of Vacuum Science \& Technology B 9, 1559 - 1561 (1991).

125. M. Nonnenmacher, M. P. O'Boyle \& H. K. Wickramasinghe: Kelvin probe force microscopy. Applied Physics Letters 58, 2921 - 2923 (1991).

126. C. Shafai, D. J. Thomson, M. Simard-Normandin, G. Mattiussi \& P. J. SCANLON: Delineation of semiconductor doping by scanning resistance microscopy. Applied Physics Letters 64, 342 - 344 (1994).

127. M. P. Murrell, M. E. Welland, S. J. O’Shea, T. M. H. Wong, J. R. Barnes, A. W. McKinnon, M. Heyns \& S. Verhaverbeke: Spatially resolved electrical measurements of $\mathrm{SiO}_{2}$ gate oxides using atomic force microscopy. Applied Physics Letters 62, 786 - 788 (1993).

128. S. J. O’Shea, R. M. Atta \& M. E. Welland: Characterization of tips for conducting atomic force microscopy. Review of Scientific Instruments 66, 2508 $2512(1995)$

129. Y. G. NAidyuk \& I. K. Yanson: Point-Contact Spectroscopy (Springer, 2005).

130. F. Houzé, R. Meyer, O. Schneegans \& L. Boyer: Imaging the local electrical properties of metal surfaces by atomic force microscopy with conducting probes. $\mathbf{6 9}$, 1975 - 1977 (1996).

131. Omicron NanoTechnology GmbH (2011). <http://www.omicron.de > (besucht am 06. 08. 2011).

132. The SCALA PRO Software Manual. 5.0. Omicron NanoTechnology GmbH (Taunusstein, 2003).

133. J. J. Wortman \& R. A. Evans: Young's Modulus, Shear Modulus, and Poisson's Ratio in Silicon and Germanium. Journal of Applied Physics 36, 153 - 156 (1965).

134. J. H. So, J. R. Gladden, Y. F. Hu, J. D. Maynard \& Q. Li: Measurements of Elastic Constants in Thin Films of Colossal Magnetoresistance Material. Physical Review Letters 90, 036103 (2003).

135. S. HÜHN. Persönliche Kommunikation (2010). 
136. W. F. Brinkman, R. C. Dynes \& J. M. Rowell: Tunneling Conductance of Asymmetrical Barriers. 41, 1915 - 1921 (1970).

137. F. Belfiore. Messung/persönliche Kommunikation (2010).

138. T. T. Tsong: Effects of an electric field in atomic manipulations. Physical Review B 44, 13703 - 13710 (1991).

139. J.-O. Krisponeit, C. Kalkert, B. Damaschke, V. Moshnyaga \& K. Samwer: Nanoscale resistance switching in manganite thin films: Sharp voltage threshold and pulse-width dependence. Physical Review B 82, 144440 (2010).

140. I. Leonov, A. I. Poteryaev, V. I. Anisimov \& D. Vollhardt: Electronic Correlations at the $\alpha-\gamma$ Structural Phase Transition in Paramagnetic Iron. Physical Review Letters 106, 106405 (2011).

141. R. Muenstermann, T. Menke, R. Dittmann, S. Mi, C.-L. Jia, D. Park \& J. MAYER: Correlation between growth kinetics and nanoscale resistive switching properties of $\mathrm{SrTiO}_{3}$ thin films. Journal of Applied Physics 108, 124504 (2010).

142. K. Szot, W. Speier, G. Bihlmayer \& R. Waser: Switching the electrical resistance of individual dislocations in single-crystalline SrTiO3. Nature Materials 5, $312-320$ (2006).

143. P. Paruch, T. Tybell \& J.-M. Triscone: Nanoscale control of ferroelectric polarization and domain size in epitaxial $\mathrm{Pb}\left(\mathrm{Zr}_{0.2} \mathrm{Ti}_{0.8}\right) \mathrm{O}_{3}$ thin films. Applied Physics Letters 79, $530-532$ (2001).

144. T. Tybell, P. Paruch, T. Giamarchi \& J.-M. Triscone: Domain Wall Creep in Epitaxial Ferroelectric $\mathrm{Pb}\left(\mathrm{Zr}_{0.2} \mathrm{Ti}_{0.8}\right) \mathrm{O}_{3}$ Thin Films. Physical Review Letters 89, 097601 (2002).

145. Y. B. Nian, J. Strozier, N. J. Wu, X. Chen \& A. Ignatiev: Evidence for an Oxygen Diffusion Model for the Electric Pulse Induced Resistance Change Effect in Transition-Metal Oxides. Physical Review Letters 98, 146403 (2007).

146. I. Yasuda, K. Ogasawara, M. Hishinuma, T. Kawada \& M. Dokiya: Oxygen tracer diffusion coefficient of (La, Sr) $\mathrm{MnO}_{3 \pm \delta}$. Solid State Ionics 86-88, $1197-1201$ (1996).

147. R. A. De Souza \& J. A. Kilner: Oxygen transport in $\mathrm{La}_{1-\mathrm{x}} \mathrm{Sr}_{\mathrm{x}} \mathrm{Mn}_{1-\mathrm{y}} \mathrm{Co}_{\mathrm{y}} \mathrm{O}_{3 \pm \delta}$ perovskites: Part I. Oxygen tracer diffusion. Solid State Ionics 106, 175 - 187 (1998).

148. L. Malavasi \& G. Flor: Oxygen Chemical Diffusion Coefficient in Manganite Thin Films by Isothermal Electric Resistivity Measurements. The Journal of Physical Chemistry B 107, 13880 - 13884 (2003).

149. S. H. Huerth, H. D. Hallen \& B. Moeckly: Spectroscopy of voltage dependence of oxygen movement in $\mathrm{YBa}_{2} \mathrm{Cu}_{3} \mathrm{O}_{7-\delta}$. Physical Review B 67, 180506 (2003).

150. H. K. Henisch: Semiconductor Contacts: An Approach to Ideas and Models (Clarendon Press, Oxford, 1984).

151. S. Richter, D. Cahen, S. R. Cohen, K. Gartsman, V. Lyakhovitskaya \& Y. Manassen: Fabrication of sub- $\mu \mathrm{m}$ bipolar transistor structures by scanning probe microscopy. Applied Physics Letters 73, 1868 - 1870 (1998). 
152. M. C. Weber, B. Hillebrands, V. Moshnyaga \& K. Samwer: Spin-lattice relaxation phenomena in manganite $\mathrm{La}_{0.7} \mathrm{Sr}_{0.3} \mathrm{MnO}_{3}$ thin films. EPL (Europhysics Letters) 73, $285-291$ (2006).

153. S. Lemerle, J. Ferré, C. Chappert, V. Mathet, T. Giamarchi \& P. Le Doussal: Domain Wall Creep in an Ising Ultrathin Magnetic Film. Physical Review Letters 80, 849 - 852 (1998).

154. A. L. Greer \& F. Spaepen: Creep, Diffusion and Structural Relaxation in Metallic Glasses. Annals of the New York Academy of Sciences 371, 218 - 237 (1981).

155. G. Blatter, M. V. Feigel'man, V. B. Geshienbein, A. I. Larkin \& V. M. VINOKUR: Vortices in high-temperature superconductors. Reviews of Modern Physics 66, $1125-1388$ (1994).

156. H. Yamada, Y. Ogawa, Y. Ishit, H. Sato, M. Kawasaki, H. Akoh \& Y. Tokura: Engineered Interface of Magnetic Oxides. Science 305, 646 - 648 (2004).

157. L. Abad, B. Martínez \& L. Balcells: Surface behavior of $\mathrm{La}_{2 / 3} \mathrm{Ca}_{1 / 3} \mathrm{MnO}_{3}$ epitaxial thin films. 87, 212502 (2005).

158. C. KALKERT: Metall-Isolator-Übergang eines (La,Pr,Ca)-Manganats - ein Vergleich von globalen und lokalen Eigenschaften. Diplomarbeit (Georg-August-Universität Göttingen, 2008).

159. X. Chen, N. J. Wu, J. Strozier \& A. Ignatiev: Direct resistance profile for an electrical pulse induced resistance change device. Applied Physics Letters 87, 233506 (2005).

160. J. Burgy, E. Dagotto \& M. Mayr: Percolative transitions with first-order characteristics in the context of colossal magnetoresistance manganites. Physical Review B 67, 014410 (2003).

161. J. Burgy, A. Moreo \& E. Dagotto: Relevance of Cooperative Lattice Effects and Stress Fields in Phase-Separation Theories for CMR Manganites. Physical Review Letters 92, 097202 (2004).

162. R. P. Rairigh, G. Singh-Bhalla, S. Tongay, T. Dhakal, A. Biswas \& A. F. HEBARD: Colossal magnetocapacitance and scale-invariant dielectric response in phase-separated manganites. Nature Physics 3, 551 - 555 (2007).

163. F. FischGRABE: AC-Transport in CMR-Manganatschichten. Diplomarbeit (GeorgAugust-Universität Göttingen, 2011).

164. P. Lunkenheimer, F. Mayr \& A. Loidl: Dynamic conductivity from audio to optical frequencies of semiconducting manganites approaching the metal-insulator transition. Annalen der Physik 15, 498 - 507 (2006).

165. Grundlagen der Elektrotechnik I, 5. Aufl. (Springer-Verlag, 1999).

166. M. Schlup: Elektrizitätslehre 1 (Vorlesungsskript). Zürcher Hochschule Winterthur (2003). <https : //home.zhaw.ch/〜spma/Scripts/ET_ST/EL1/Theorie/EL1_Th 5.pdf $>$ (besucht am 30.10.2011). 
167. N. Metropolis, A. W. Rosenbluth, M. N. Rosenbluth, A. H. Teller \& E. Teller: Equation of State Calculations by Fast Computing Machines. The Journal of Chemical Physics 21, 1087 - 1092 (1953).

168. M. E. J. Newman \& G. T. Barkema: Monte Carlo Methods in Statistical Physics (Oxford University Press, 2002). 


\section{Veröffentlichungen}

\section{Artikel}

- „Nanoscale resistance switching in manganite thin films: Sharp voltage threshold and pulse-width dependence",

J.-O. Krisponeit, C. Kalkert, B. Damaschke, V. Moshnyaga und K. Samwer, Physical Review B 82, 144440 (2010).

- "Resistive switching at manganite/manganite interfaces",

C. Kalkert, J.-O. Krisponeit, M. Esseling, O. I. Lebedev, V. Moshnyaga, B. Damaschke, G. van Tendeloo und K. Samwer,

Applied Physics Letters 99, 132512 (2011).

\section{Konferenzbeiträge}

- Frühjahrstagung der Deutschen Physikalischen Gesellschaft, Regensburg (2010), "Switching on the Nanoscale on $\mathrm{La}_{0.7} \mathrm{Ca}_{0.3} \mathrm{MnO}_{3}$ Thin Films",

J.-O. Krisponeit, C. Kalkert, B. Damaschke, V. Moshnyaga und K. Samwer.

- Frühjahrstagung der Deutschen Physikalischen Gesellschaft, Regensburg (2010), „Resistive switching in nanocolumnar manganite thin films structured with e-beam lithography",

C. Kalkert, M. Esseling, J.-O. Krisponeit , V. Moshnyaga, B. Damaschke und K. Samwer.

- Frühjahrstagung der Deutschen Physikalischen Gesellschaft, Dresden (2011), „Resistive switching on $\mathrm{La}_{0.8} \mathrm{Ca}_{0.2} \mathrm{MnO}_{3}$ films: nanoscale and time evolution studies of conductively switched domains",

J.-O. Krisponeit, C. Kalkert, B. Damaschke, V. Moshnyaga und K. Samwer. 
- Frühjahrstagung der Deutschen Physikalischen Gesellschaft, Dresden (2011), „Resistive switching in nanocolumnar manganite thin films ",

C. Kalkert, J.-O. Krisponeit, V. Moshnyaga, B. Damaschke und K. Samwer.

- Frühjahrstagung der Deutschen Physikalischen Gesellschaft, Dresden (2011), "AC electric transport in CMR-manganite thin films",

F. Fischgrabe, S. Hühn, J.-O. Krisponeit, K. Samwer und V. Moshnyaga. 


\section{Danksagung}

An dieser Stelle möchte ich all jenen meine Dankbarkeit zum Ausdruck bringen, welche direkt oder indirekt zum Gelingen dieser Arbeit beitrugen. Herrn Prof. Dr. Konrad Samwer danke ich für das Angebot der Promotionsstelle am I. Physikalischen Institut in Göttingen und die sehr freundliche Aufnahme. Trotz seines vollen Terminplans fand er die Zeit für ein enges Betreuungsverhältnis, ließ aber auch genug Spielraum für eigene Ideen. Seine Freude am gemeinschaftlichen Diskutieren der Ergebnisse und Interpretationen war eine große Hilfe. Herrn Prof. Dr. Christian Jooss danke ich für die Übernahme des Koreferates. Auch er hat durch die Diskussionen im gemeinsamen Manganate-Seminar und bei anderen Gelegenheiten zum Gelingen der Arbeit beigetragen.

Ohne Dr. Bernd Damaschke und seine kontinuierliche Betreuung, insbesondere während unseres gemütlichen montäglichen Jour Fixe, wäre diese Arbeit so nicht möglich gewesen. Sein humorvolles und diplomatisches Wesen sowie das gründliche Korrekturlesen dieser Arbeit waren sehr hilfreich. Prof. Dr. Vasily Moshnyaga trug durch seine große Diskussionsfreude und sein schier unerschöpfliches Wissen über die Manganate sehr zu dieser Arbeit bei. Auch für die vielen aufmunternden Worte danke ich sehr.

Die enge Zusammenarbeit mit Christin Kalkert hat mir stets große Freude bereitet. Ich danke für die unzähligen ergiebigen Diskussionen, auch über vermeintlich Offensichtliches. Diese Arbeit hat sehr von den gegenseitigen Ratschlägen und Ergänzungen profitiert. Ihr, aber auch dem „Dreieck“ - Markus Jungbauer, Markus Michelmann sowie Sebastian Hühn - und Florian Fischgrabe danke ich für das hervorragende Arbeitsklima innerhalb der Manganategruppe und den Spaß an vielen gemeinsamen Abenden.

Uta Fillipichs herzliche Art machte es zu einem Vergnügen, mit ihr das Büro zu teilen. Auch als externer „Theoretiker" kam ich mir gleich willkommen vor. Ihre Hilfsbereitschaft und Anteilnahme haben meine Arbeit sehr bereichert. Carsten Mahn danke ich ebenfalls für die hervorragende Unterstützung in allen technischen Belangen. Wie er es geschafft hat, sich trotz seines chronischen Zeitmangels so gelassen jedem ihm angetragenen Problem zu widmen und immer überzeugende und meist bestechend einfache Lösungen zu finden, bleibt mir ein Rätsel. 
All den anderen, hier namentlich nicht aufgeführten Mitgliedern der Manganate-Gruppe und des I. Physikalischen Instituts möchte ich für die sehr angenehme Atmosphäre danken. Natürlich bleibe ich dabei auch den ehemaligen Institutsmitgliedern in Dankbarkeit verbunden, ohne deren Ratschläge und Anregungen diese Arbeit so nicht gelungen wäre.

Meinen Eltern danke ich dafür, dass sie mir das Studium ermöglichten und mir während der Promotion aus der Ferne soviel Rückhalt gaben. Die viel zu seltenen Besuche in der Heimat waren jedesmal wie ein kleiner Urlaub für mich.

Der größte Dank gebührt meiner Freundin Verena Bendel. Ihre Aufmunterung selbst aus der Ferne, ihr Verständnis gegenüber meiner Zeitnot und Anspannung, und die Gewißheit ihrer Liebe gaben mir täglich Kraft. Ihre aufopfernde, akribische Suche nach Fehlern aller Art beim Korrekturlesen waren der Qualität dieser Arbeit äußerst zuträglich. Diese Dissertation wäre nicht entstanden, hätte sie mich nicht nach Göttingen gelockt - ich danke ihr für die schönen Jahre von Herzen.

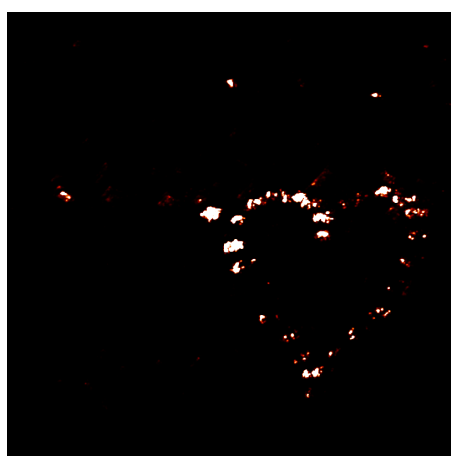




\section{Lebenslauf}

\section{Persönliche Daten}

Name:

Geburtsdatum:

Geburtsort:

Familienstand:

Staatsangehörigkeit:

Schulbildung

$1989-1993$

$1993-1995$

$1995-2002$

2002

\section{Studium}

$2002-2008$

2008

seit 2008
Jon-Olaf Krisponeit

5. Oktober 1982

Bremen

ledig

deutsch

Grundschule Falkenberg

Orientierungsstufe Lilienthal

Gymnasium Lilienthal

Abitur

Physikstudium an der Universität Bremen

Diplomarbeit bei Prof. Dr. Gerd Czycholl:

Numerische Modellierung eines Einzelmolekültransistors

Wissenschaftlicher Mitarbeiter am I. Physikalischen Institut der

Georg-August-Universität Göttingen 